Supplement of SOIL, 7, 305-332, 2021

https://doi.org/10.5194/soil-7-305-2021-supplement

(c) Author(s) 2021. CC BY 4.0 License.

(c) (i)

Supplement of

\title{
Continental-scale controls on soil organic carbon across sub-Saharan Africa
}

Sophie F. von Fromm et al.

Correspondence to: Sophie F. von Fromm (sfromm@bgc-jena.mpg.de)

The copyright of individual parts of the supplement might differ from the article licence. 


\title{
R Code: Continental-scale controls on soil organic carbon across sub-Saharan Africa
}

\author{
Sophie F. von Fromm
}

$03 / 31 / 2021$

\section{AfSIS data}

This document contains all the $\mathrm{R}$ code that were used to generate the statistical analysis and all figures for the manuscript von Fromm et al. (2021): Continental-scale controls on soil organic carbon across sub-Saharan Africa. If you have questions please contact Sophie F. von Fromm (sfromm@bgc-jena.mpg.de). The document also contains several links to websites from where some of the code used here have been adapted.

Note: For figures, only the code is provided. The figures themselves are shown in the manuscript and in the SI of the manuscript.

The following $\mathrm{R}$ packages are needed for the code:

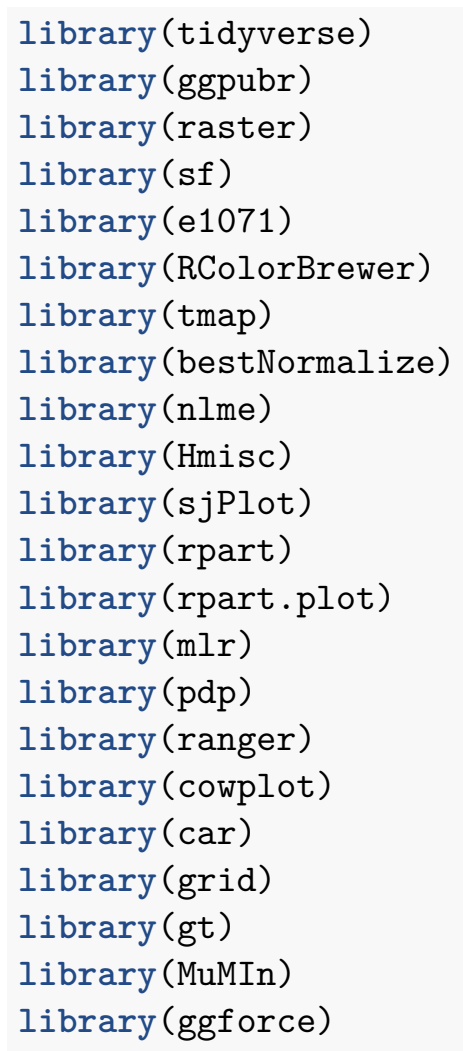


Create own theme for making figures with ggplot:

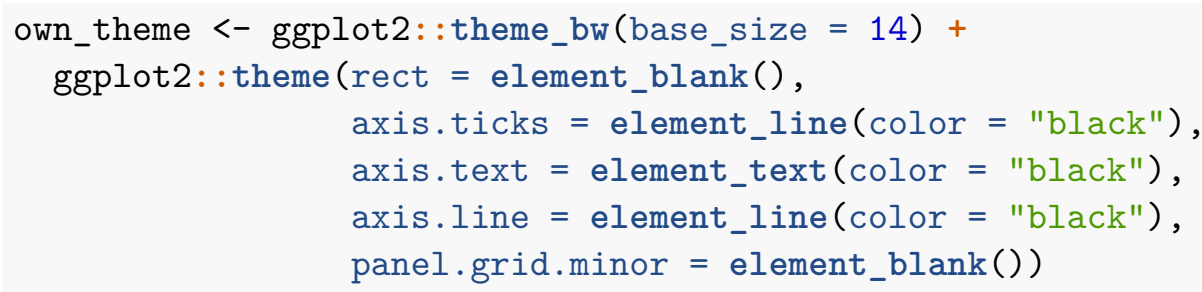

The data used in this project is from the African Soil Information Service (AfSIS: http://africasoils. net/) which is lead and maintained by the International Centre for Research in Agroforestry, Nairobi, Kenya. The lab meaurements were led by Steve P. McGrath and Stephan M. Haefele at the Department of Sustainable Agriculture Sciences, Rothamsted Research, Harpenden UK. For more information see also the Methods in von Fromm et al (2021).

\section{Overview:}

The following section gives a short overview about the data used for the publication. 47 variables and 2002 samples are included in the data file:

\begin{tabular}{|c|c|c|c|c|c|}
\hline [1] & "SSN" & "Longitude" & "Latitude" & "Region" & "Country" \\
\hline [6] & "Site" & "Cluster" & "Profile" & "Depth" & "Clay_2um" \\
\hline$[11]$ & "Clay_8um" & "Clay_20um" & "Total_C" & "CORG" & "CINORG" \\
\hline [16] & "Total_N" & "pH" & "Am_Ox_Al" & "Am_Ox_Fe" & "Am_Ox_Mn" \\
\hline [21] & "Am_Ox_P" & "Olsen_P" & "pbi" & "Caex" & "Kex" \\
\hline [26] & "Mgex" & "Naex" & "eCEC" & "Al" & "Ca" \\
\hline [31] & "Co" & "Cr" & "Cu" & "Fe" & "K" \\
\hline [36] & "Mg" & "Mn" & "Na" & "Ni" & "P" \\
\hline [41] & "Pb" & "S" & "Zn" & "Total_PSD" & "VegStructure" \\
\hline \#\# [46] & "PlotCultMgd" & "Notes" & & & \\
\hline
\end{tabular}

\section{Short explanation of the variables included in the dataset:}

SSN - Sample code

Longitude/Latitude - Longitude/Latitude of sample location

Region - Sampling area within sub-Saharan Africa

Country - Country of origin of samples

Site - Site name

Cluster - Cluster number within each site

Profile - Profile number within each cluster

Depth - Sampling depth (categorial; topsoil: 0-20cm, subsoil: $20-50 \mathrm{~cm}$ )

Clay_2um - All particles $<8 \mu \mathrm{m}[\%]$

Clay_8um - All particles $<8 \mu \mathrm{m}[\%]$

Clay_20um - All particles $<8 \mu \mathrm{m}[\%]$

Total_C, CORG, CINORG - Total, organic, inorganic C content [wt-\%]

Total_N - Total N content [wt-\%]

$\mathrm{pH}-$ Soil $\mathrm{pH}_{\mathrm{H} 2 \mathrm{O}}$

Am_Ox_Al/Fe/Mn/P - Amorphous oxalate aluminum/iron/manganese/phosphorus [mg/kg]

Olsen_P - Sodium bicarbonate extraction of available P [mg/kg] 
pbi - Phosphorus buffer index

Caex, Kex, Mgex, Naex - Exchangeable Ca, K, Mg, Na [cmol $\left.{ }^{+} / \mathrm{kg}\right]$

eCEC - Effective cation exchange capacity

$\mathrm{Al}-\mathrm{Zn}$ - Element concentration (aqua regia) $[\mathrm{mg} / \mathrm{kg}]$

Total_PSD - Total particle size distribution [\%]

VegStructure - Land-cover (based on LDSF field data)

PlotCultMgd - Is the plot cultivated? Yes/No

Notes - Field notes

Missing values within the data:

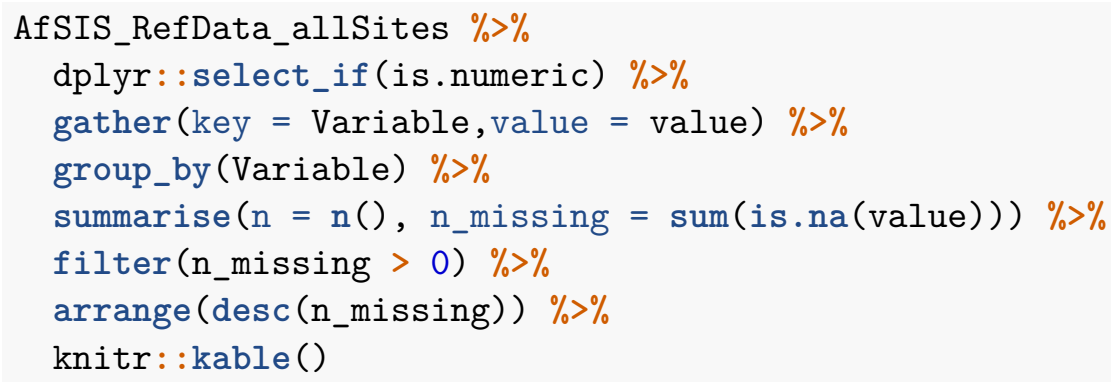

\begin{tabular}{lrr}
\hline Variable & $\mathrm{n}$ & $\mathrm{n}$ _missing \\
\hline Clay_20um & 2002 & 363 \\
Clay_2um & 2002 & 363 \\
Clay_8um & 2002 & 363 \\
Total_PSD & 2002 & 363 \\
PlotCultMgd & 2002 & 159 \\
pH & 2002 & 30 \\
Total_C & 2002 & 10 \\
Total_N & 2002 & 10 \\
CINORG & 2002 & 4 \\
CORG & 2002 & 4 \\
\hline
\end{tabular}

Negative values within the data:

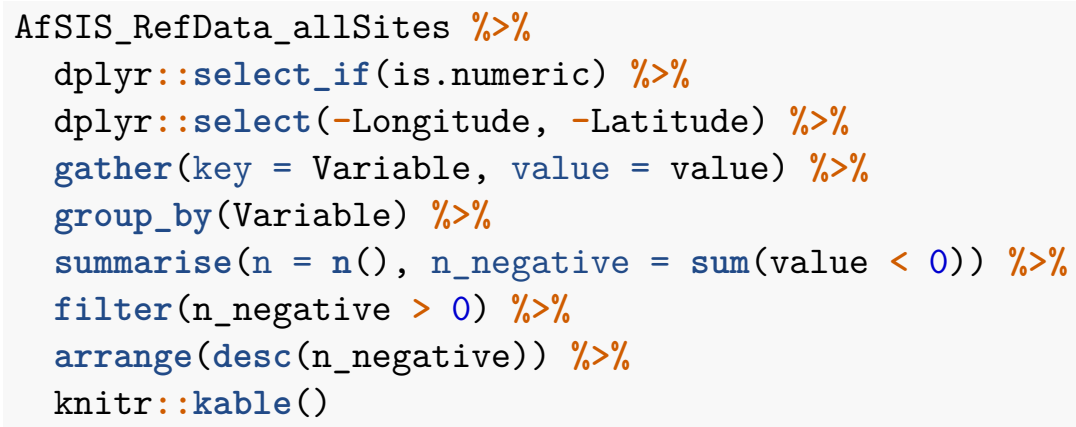




\begin{tabular}{lrr}
\hline Variable & $\mathrm{n}$ & $\mathrm{n}$ _negative \\
\hline Am_Ox_P & 2002 & 493 \\
Naex & 2002 & 145 \\
eCEC & 2002 & 123 \\
$\mathrm{~Pb}$ & 2002 & 7 \\
$\mathrm{Cu}$ & 2002 & 6 \\
$\mathrm{P}$ & 2002 & 4 \\
Caex & 2002 & 2 \\
Kex & 2002 & 1 \\
Mgex & 2002 & 1 \\
\hline
\end{tabular}

\section{Global data}

For the statistical analyses the AfSIS data was paired with global data (MAP, MAT, PET, land cover (only for gap-filling)). The follwing section provides the code for extracting the global data and merging it with the AfSIS dataset.

Note: You need to download and store the global data beforehand on your computer. Links to the data sources are provided below.

\subsection{Climate data}

Data sources:

WorldClim: http://worldclim.org/version2 and

PET: https://figshare.com/articles/Global_Aridity_Index_and_Potential_Evapotranspiration_ET0_ Climate_Database_v2/7504448/3

MAT (= Mean annual temperature) raster:

MAT_directory <- "D:/Sophie/PhD/AfSIS_GlobalData/wc2.0_30s_bio/wc2.0_bio_30s_01.tif"

MAT_raster <- raster: :raster(MAT_directory)

MAT <- raster: :extract(MAT_raster, cbind(AfSIS_RefData_allSites\$Longitude, AfSIS_RefData_allSites\$Latitude))

MAP (= Mean annual precipitation) raster:

MAP_directory <- "D:/Sophie/PhD/AfSIS_GlobalData/wc2.0_30s_bio/wc2.0_bio_30s_12.tif"

MAP_raster <- raster: :raster(MAP_directory)

MAP <- raster: :extract(MAP_raster, cbind(AfSIS_RefData_allSites\$Longitude,

AfSIS_RefData_allSites\$Latitude))

PET (= Potential annual evapotranspiration) raster: 
PET_directory <- "D:/Sophie/PhD/AfSIS_GlobalData/global-et0_annual.tif/et0_yr/et0_yr.tif"

PET_raster <- raster: :raster(PET_directory)

PET <- raster: :extract(PET_raster, cbind(AfSIS_RefData_allSites\$Longitude,

AfSIS_RefData_allSites\$Latitude))

Merge AfSIS data with extracted climate data:

AfSIS_RefData_allSites_Clima <- cbind(AfSIS_RefData_allSites, MAT, MAP, PET)

Calculating Aridity Index (PET/MAP):

AfSIS_RefData_allSites_Clima <- AfSIS_RefData_allSites_Clima \% $\%$

dplyr: : mutate (AridityIndex $=$ PET/MAP)

\subsection{Monthly climate data}

Data sources:

MAP: https://www.worldclim.org/data/worldclim21.html) and

PET: https://cgiarcsi.community/data/global-aridity-and-pet-database/

MAP (= monthly mean annual precipitation) raster

1 - January:

MAP_1_directory <- "D:/Sophie/PhD/AfSIS_GlobalData/wc2.1_30s_prec/wc2.1_30s_prec_01.tif"

MAP_1_raster <- raster: :raster(MAP_1_directory)

MAP_1 <- raster: :extract(MAP_1_raster, cbind(AfSIS_RefData_allSites_Clima\$Longitude, AfSIS_RefData_allSites_Clima\$Latitude))

\section{2 - February:}

MAP_2_directory <- "D:/Sophie/PhD/AfSIS_GlobalData/wc2.1_30s_prec/wc2.1_30s_prec_02.tif"

MAP_2_raster <- raster: :raster(MAP_2_directory)

MAP_2 <- raster: :extract(MAP_2_raster, cbind(AfSIS_RefData_allSites_Clima\$Longitude, AfSIS_RefData_allSites_Clima\$Latitude))

Code for the other months/variables are not shown since they are always the same.

Merge AfSIS data with extracted climate data and calculate monthly wetness index (MAP/PET):

AfSIS_RefData_allSites_Clima_month <- cbind(AfSIS_RefData_allSites_Clima, MAP_1, MAP_2, MAP_3, MAP_4, MAP_5, MAP_6, MAP_7, MAP_8, MAP_9, MAP_10, MAP_11, MAP_12, PET_1, PET_2, PET_3, PET_4, PET_5, PET_6, $\mathrm{PET}$ _7, $\mathrm{PET}$ _8, PET_9, PET_10, PET_11, PET_12) 
AfSIS_RefData_allSites_Clima_month <- AfSIS_RefData_allSites_Clima_month $\%>\%$

dplyr: :mutate(WetnessIndex_1 = MAP_1/PET_1, WetnessIndex_2 $=$ MAP_2/PET_2, WetnessIndex_3 $=$ MAP_3/PET_3, WetnessIndex_4 = MAP_4/PET_4, WetnessIndex_5 = MAP_5/PET_5, WetnessIndex_6 $=$ MAP_6/PET_6, WetnessIndex_7 $=$ MAP_7/PET_7, WetnessIndex_8 $=$ MAP_8/PET_8, WetnessIndex_9 = MAP_9/PET_9, WetnessIndex_10 = MAP_10/PET_10, WetnessIndex_11 = MAP_11/PET_11, WetnessIndex_12 = MAP_12/PET_12) $\%>\%$

dplyr: :select (-c(MAP_1:PET_12))

\subsection{Africa land cover data}

Source: http://www.esa.int/ESA_Multimedia/Images/2017/10/African_land_cover

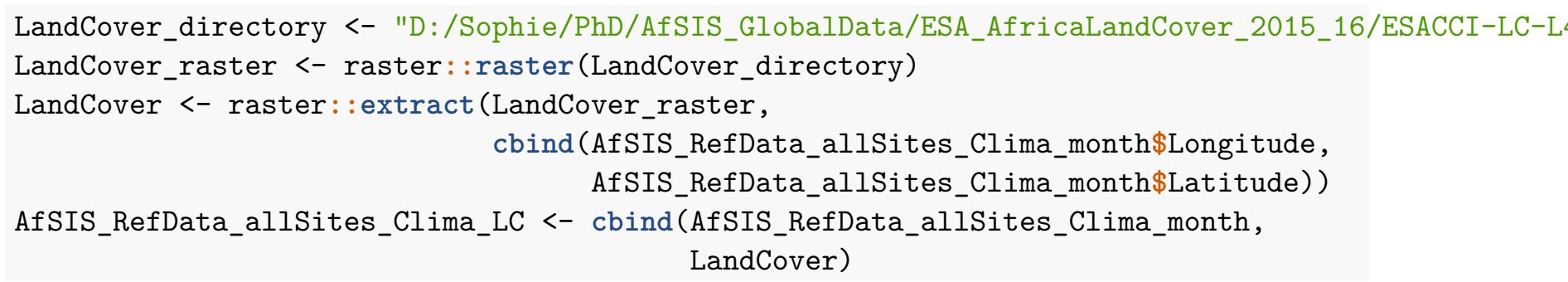

Re-name land cover code:

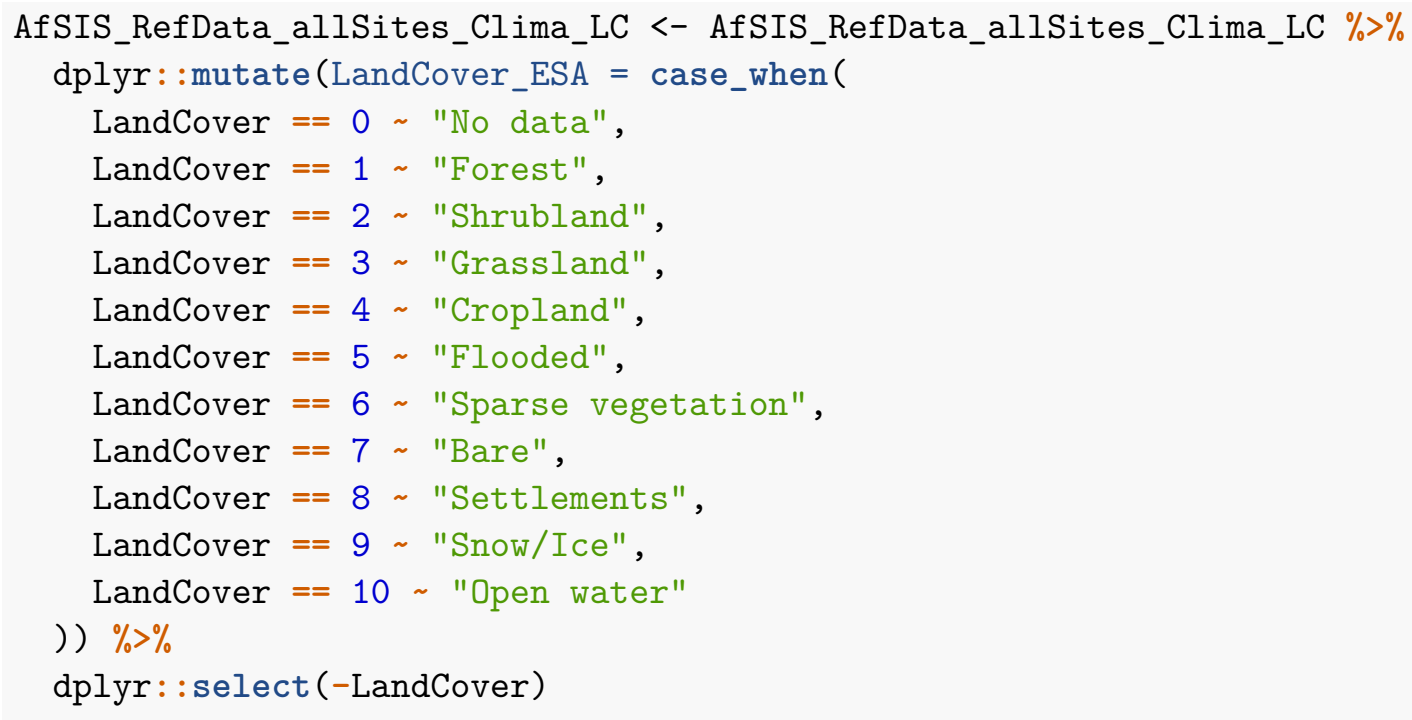


\#Save final (global) dataset

\#write_csv(AfSIS_RefData_allSites_Clima_LC, "AfSIS_RefData_Roth_allSites_GlobalData.csv")

\section{Preparation of data for analysis}

The following chapter provides all the code in order to prepare the data for the actual analyses. This includes converting units and the calculation of the CIA (weathering), gap-filling and re-classification of the land cover data, excluding missing and negative values, as well as creating groups based on seasonality, $\mathrm{pH}_{\mathrm{H} 2 \mathrm{O}}$, and $\mathrm{CIA}$ for the modelling.

Rename and filter for columns of interest.

Convert mg/kg in wt-\%: (Al, Fe, Ca, K, Mg, Mn, Na, Al_ox, Fe_ox)/10000 = wt-\%. Calculate the chemical index of alteration (CIA). For more details see Methods in von Fromm et al. (2021).

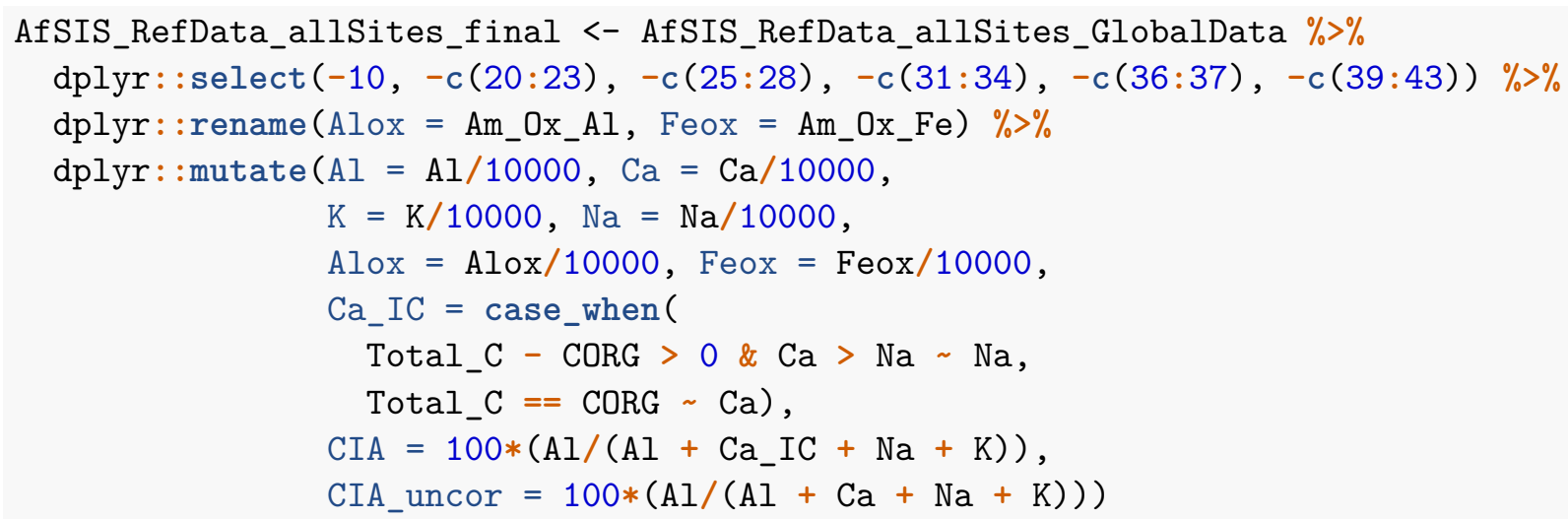

Compare uncorrected CIA and corrected CIA:

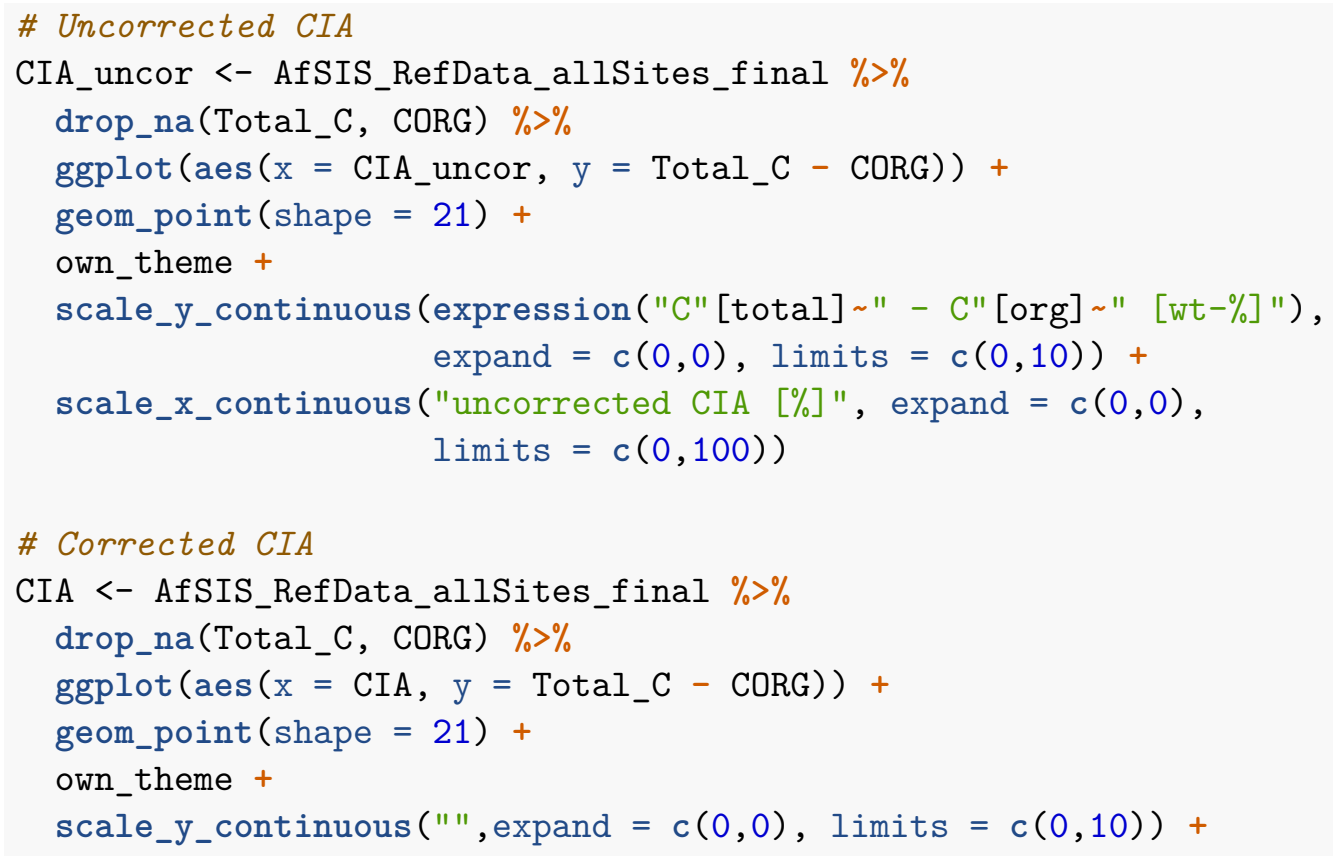




$$
\begin{gathered}
\text { scale_x_continuous }(" \text { corrected CIA }[\%] " \text {, expand }=c(0,0), \\
\text { limits }=c(0,100))
\end{gathered}
$$

\# Plot both together

ggpubr: :ggarrange(CIA_uncor, CIA)

\#ggsave("AfSIS_allData_CIA_correction.pdf", height $=4$, width $=8, d p i=300$ )

The corrected $\mathrm{CIA}$ (see Figure A2 in the manuscript) does not show an obvious trend with inorganic $\mathrm{C}$ content anymore. The corrected $\mathrm{CIA}$ will be used for all statistical analyses and only named CIA from here on.

\subsection{Land cover}

This section provides the code for gap-filling the land-cover data with the product from ESA and for the re-classification of the land cover groups.

Replace missing land cover data with ESA product:

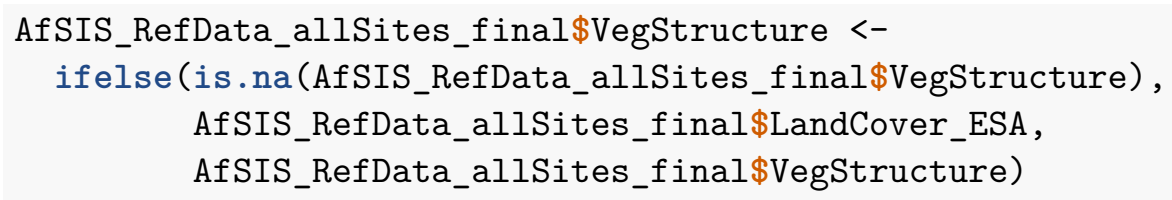

Merge land cover data into four groups: Forest, Cropland, Grassland, and Other (including mainly shrubland, bushland, and woodland):

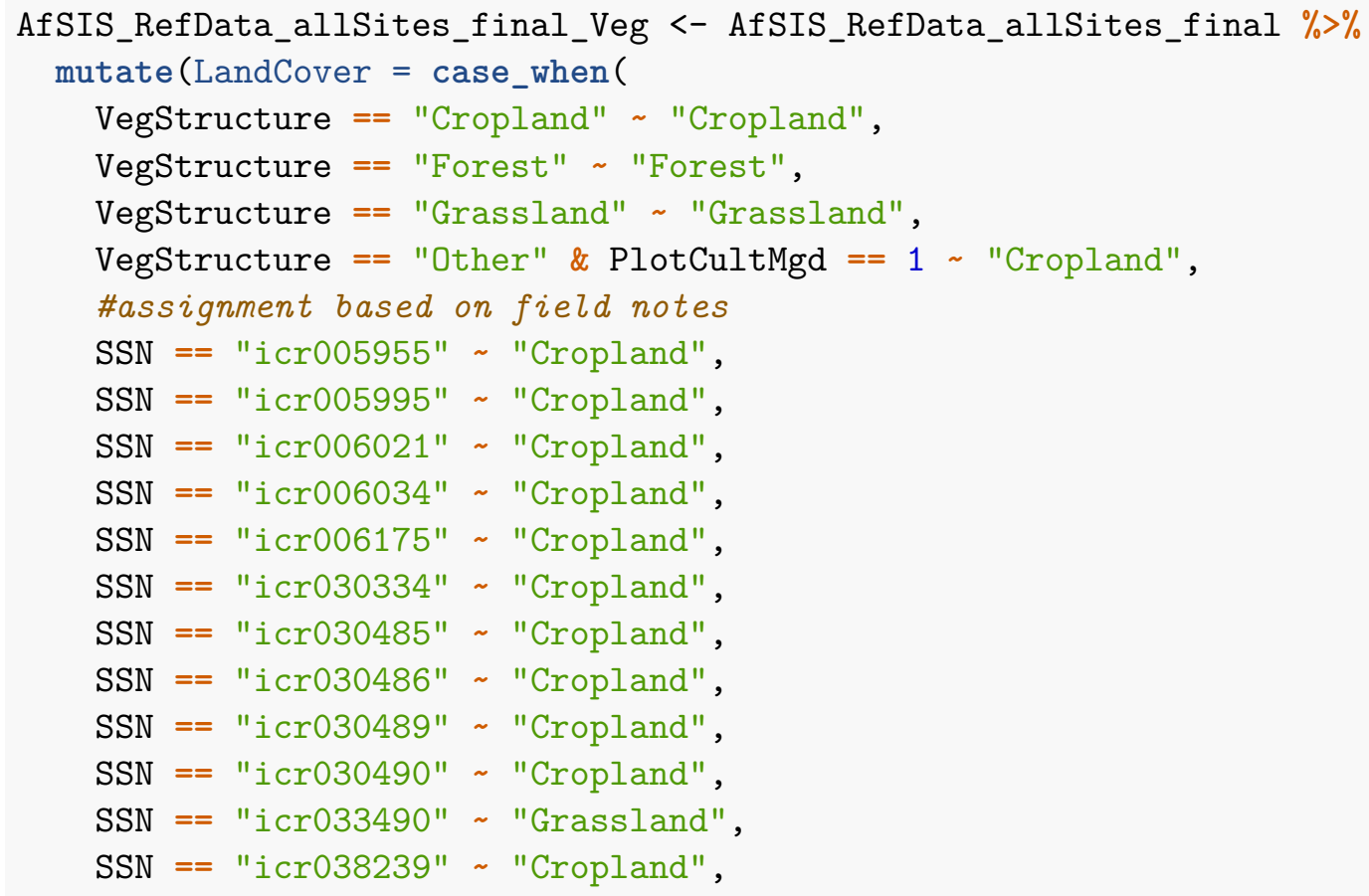




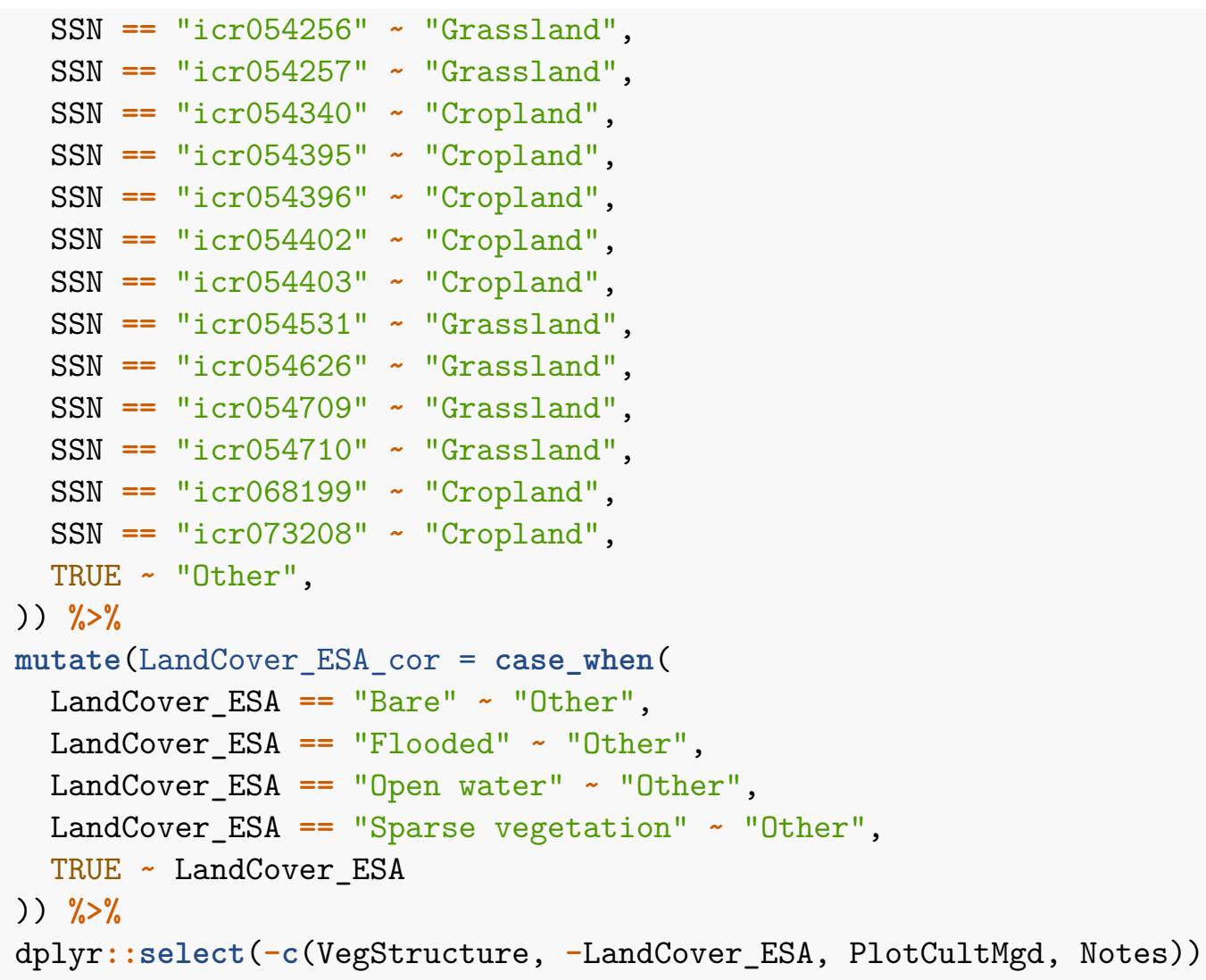

\subsection{Filtering}

This section contains the code for filtering the data, so that only meaningful (e.g., no NA's, no negative or unrealistic) values are included in the final dataset for further analysis.

Drop samples with missing values:

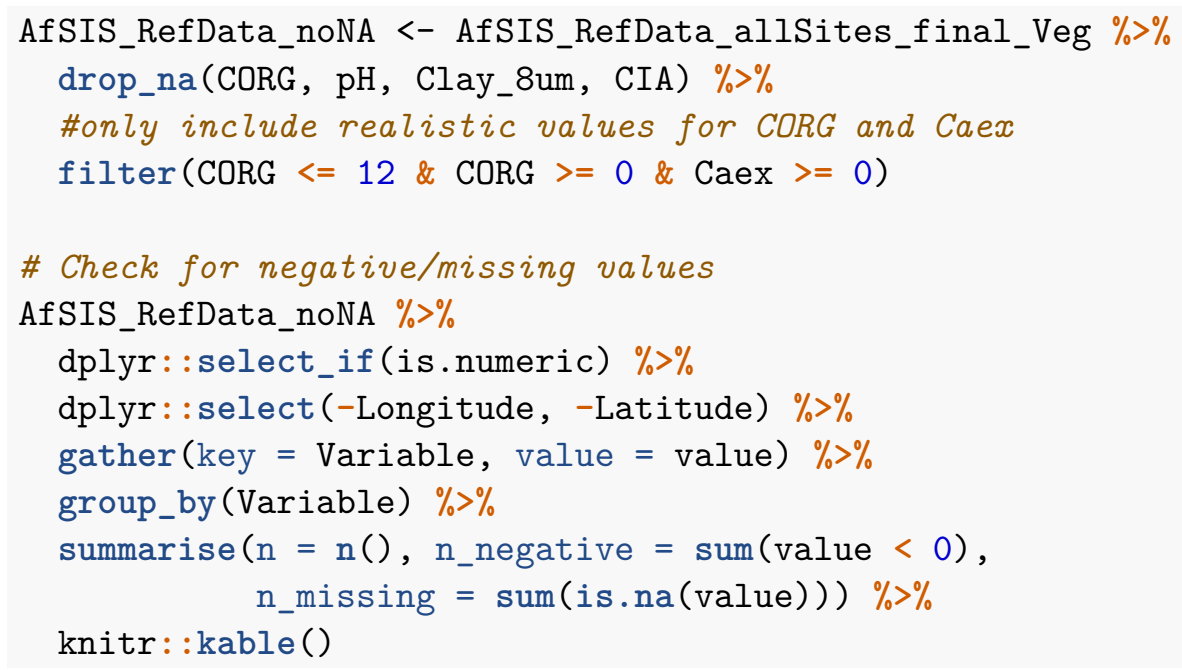




\begin{tabular}{lrrr}
\hline Variable & $\mathrm{n}$ & $\mathrm{n}$ _negative & $\mathrm{n}$ _missing \\
\hline Al & 1601 & 0 & 0 \\
Alox & 1601 & 0 & 0 \\
AridityIndex & 1601 & 0 & 0 \\
Ca & 1601 & 0 & 0 \\
Ca_IC & 1601 & 0 & 0 \\
Caex & 1601 & 0 & 0 \\
CIA & 1601 & 0 & 0 \\
CIA_uncor & 1601 & 0 & 0 \\
CINORG & 1601 & 0 & 0 \\
Clay_20um & 1601 & 0 & 0 \\
Clay_8um & 1601 & 0 & 0 \\
Cluster & 1601 & 0 & 0 \\
CORG & 1601 & 0 & 0 \\
Feox & 1601 & 0 & 0 \\
K & 1601 & 0 & 0 \\
MAP & 1601 & 0 & 0 \\
MAT & 1601 & 0 & 0 \\
Na & 1601 & 0 & 0 \\
PET & 1601 & 0 & 0 \\
pH & 1601 & 0 & 0 \\
Profile & 1601 & 0 & 0 \\
Total_C & 1601 & 0 & 0 \\
Total_N & 1601 & 0 & 0 \\
Total_PSD & 1601 & 0 & 0 \\
WetnessIndex_1 & 1601 & 0 & 0 \\
WetnessIndex_10 & 1601 & 0 & 0 \\
WetnessIndex_11 & 1601 & 0 & 0 \\
WetnessIndex_12 & 1601 & 0 & 0 \\
WetnessIndex_2 & 1601 & 0 & 0 \\
WetnessIndex_3 & 1601 & 0 & 0 \\
WetnessIndex_4 & 1601 & 0 & 0 \\
WetnessIndex_5 & 1601 & 0 & 0 \\
WetnessIndex_6 & 1601 & 1601 & 0 \\
WetnessIndex_7 & 1601 & 0 & 0 \\
WetnessIndex_8 & 1601 & 0 & 0 \\
WetnessIndex_9 & 1601 & 0 \\
\hline
\end{tabular}

Overall, 1601 samples of the AfSIS reference dataset are available for further analysis. None of the selected variables contain negative values anymore.

\subsection{Seasonality}

For modelling, the data is grouped by the monthly wetness index to create more homogenous groups based on their climate. 
Count number of wet months (based on monthly wetness index). Calculate mean for each month for each site and classify water regime:

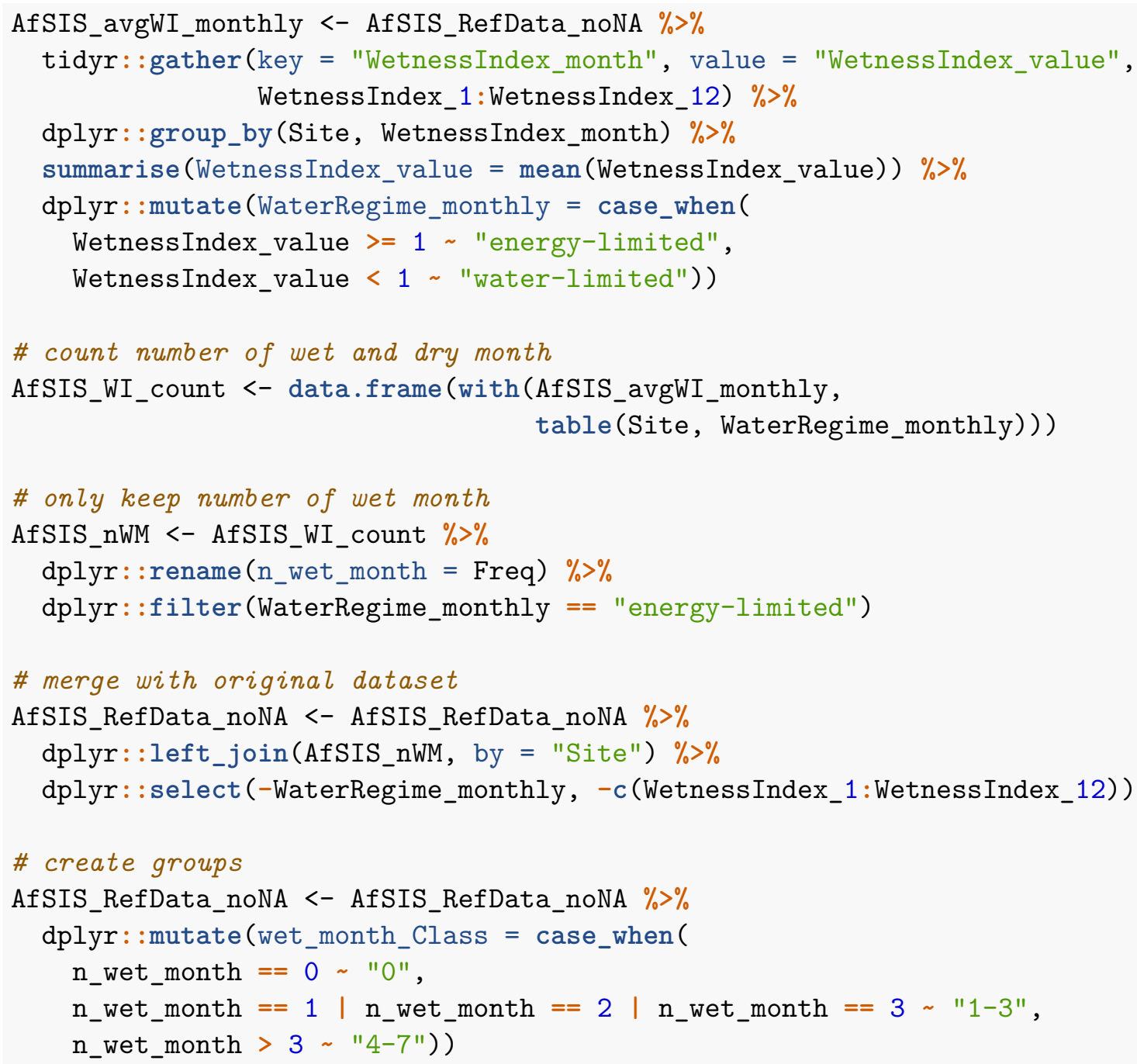

\section{$3.4 \mathrm{pH}_{\mathrm{H} 2 \mathrm{O}}$}

For modelling, the data is grouped by the numeric $\mathrm{pH}_{\mathrm{H} 20}$ values to create more homogenous groups based on their $\mathrm{pH}_{\mathrm{H} 20}$ values.

Group soil $\mathrm{pH}_{\mathrm{H} 2 \mathrm{O}}$ into four groups with equal number of samples within each group.

table(ggplot2: : cut_number(AfSIS_RefData_noNA\$pH, 4)) \# good group size + intervals

\#\#

\#\# [3.89,5.22] $(5.22,6.1] \quad(6.1,7.52] \quad(7.52,9.92]$

$\begin{array}{lllll}\text { \#\# } & 404 & 399 & 398 & 400\end{array}$ 
AfSIS_RefData_noNA $\$ \mathrm{pH} \_C l a s s<-$ as.numeric (ggplot2: :cut_number (AfSIS_RefData_noNA $\$ \mathrm{pH}, 4$ ))

Convert class numbers into meaningful pH classes:

AfSIS_RefData_noNA $\$ \mathrm{pH}$ _Class[AfSIS_RefData_noNA $\left.\$ \mathrm{pH} \_C l a s s==1\right]<-$ "strongly acidic" AfSIS_RefData_noNA $\$ \mathrm{pH}$ _Class[AfSIS_RefData_noNA $\$ \mathrm{pH}$ _Class == 2] <- "moderately acidic"

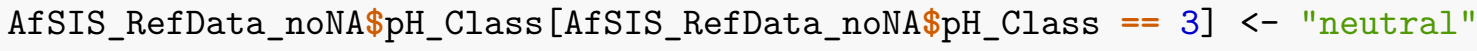
AfSIS_RefData_noNA $\$ \mathrm{pH}$ _Class[AfSIS_RefData_noNA $\left.\$ \mathrm{pH} \_C l a s s==4\right]<-$ "alkaline"

\subsection{CIA}

For modelling, the data is grouped by the numeric CIA (Chemical Index of Alteration) values to create more homogenous groups based on their CIA values.

Group CIA data into two groups with equal number of samples within each group.

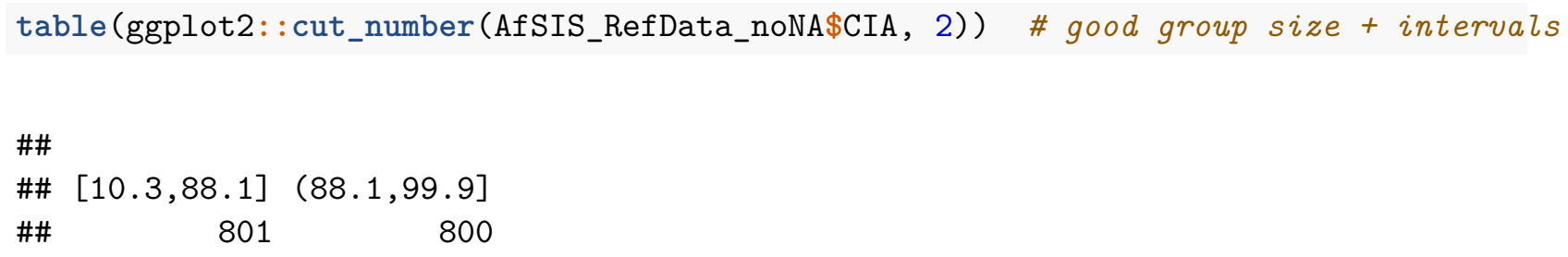

Convert class numbers into meaningful classes:

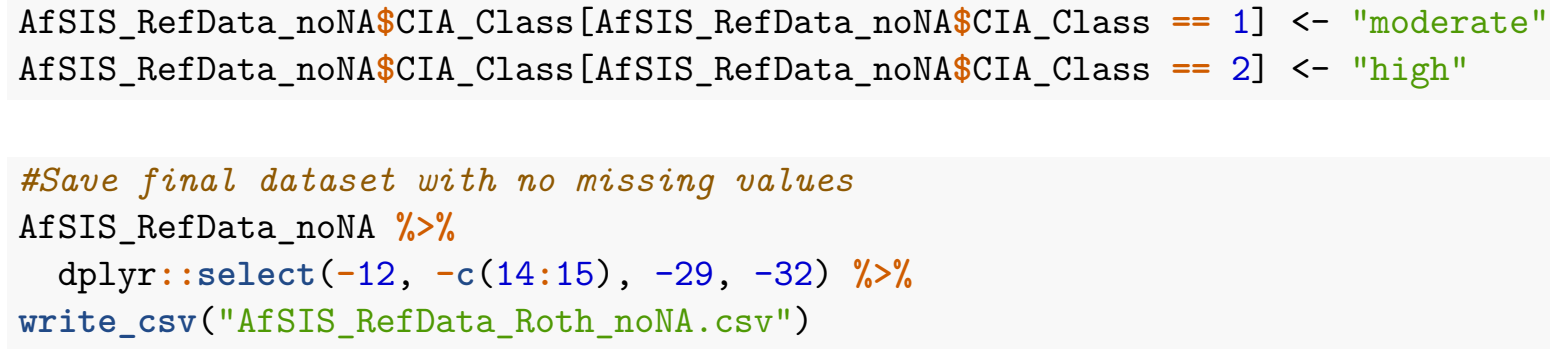

\section{Sampling locations}

This chapter contains the code to plot the sampling locations with the R package tmap, including an aridity map (PET/MAP) as a background map and one detailed overview map from one site (Didy, Madagascar) as an example for the sampling scheme.

Convert dataset into spatial format: 


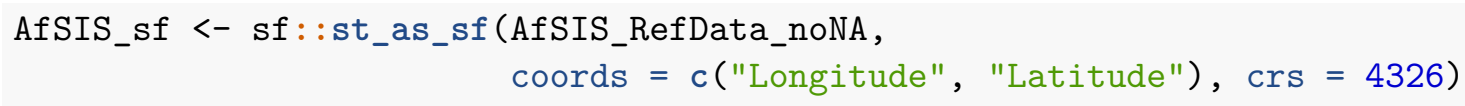

Extract map of Africa:

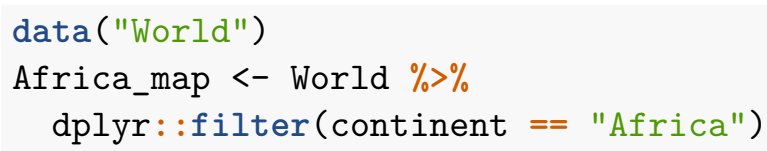

\subsection{Aridity index map}

The aridity map used here is based on the same global products used in the Global data chapter and calculated for the African continent.

Create boundaries for African contintent (based on Long/Lat) and convert into spatial format:

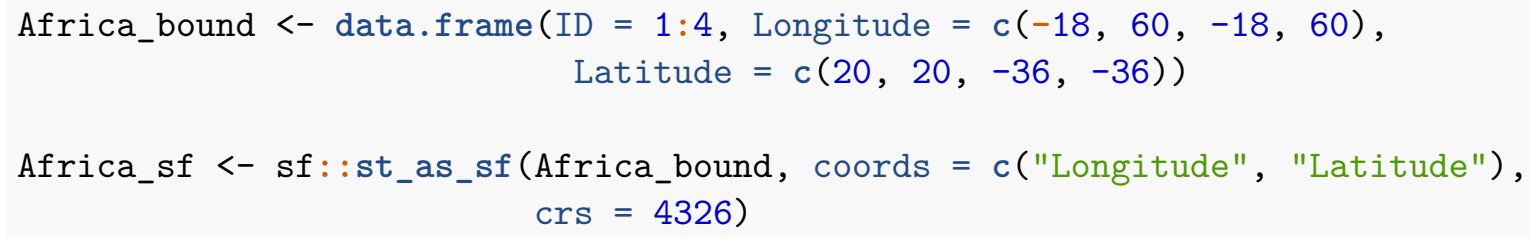

Load climate data and crop global maps based on Africa boundaries. Calculate Aridity Index (PET/MAP) for Africa:

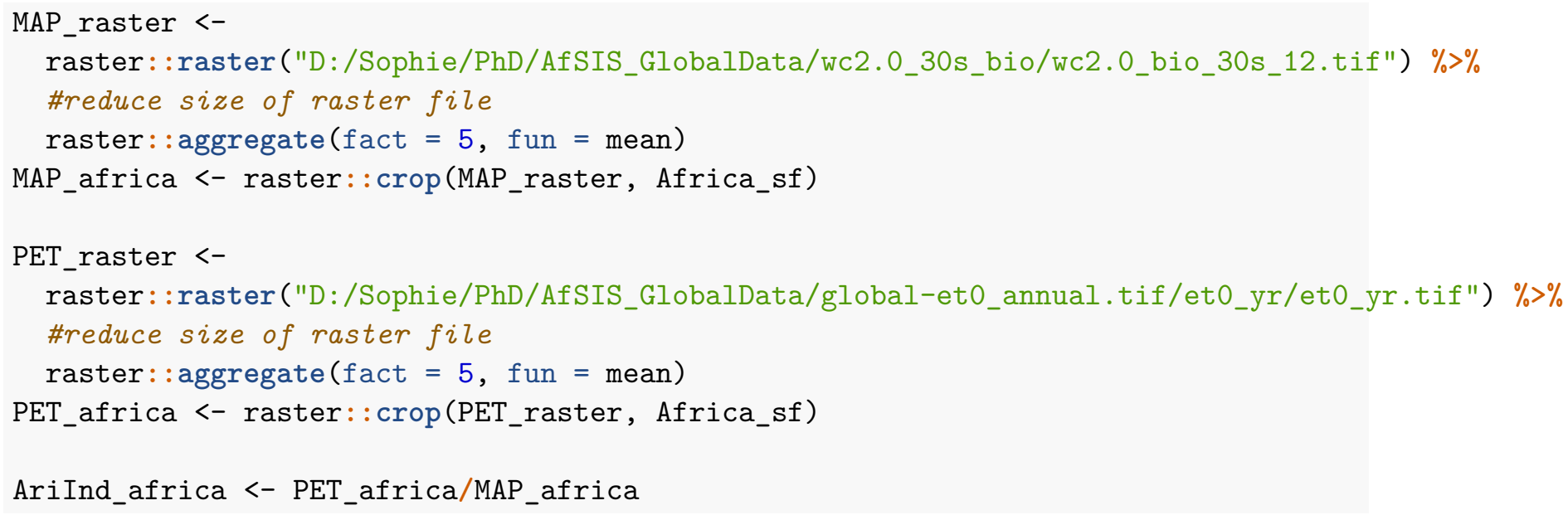

\subsection{Mapping}

The final map can be found in the manuscript (Figure 1). 


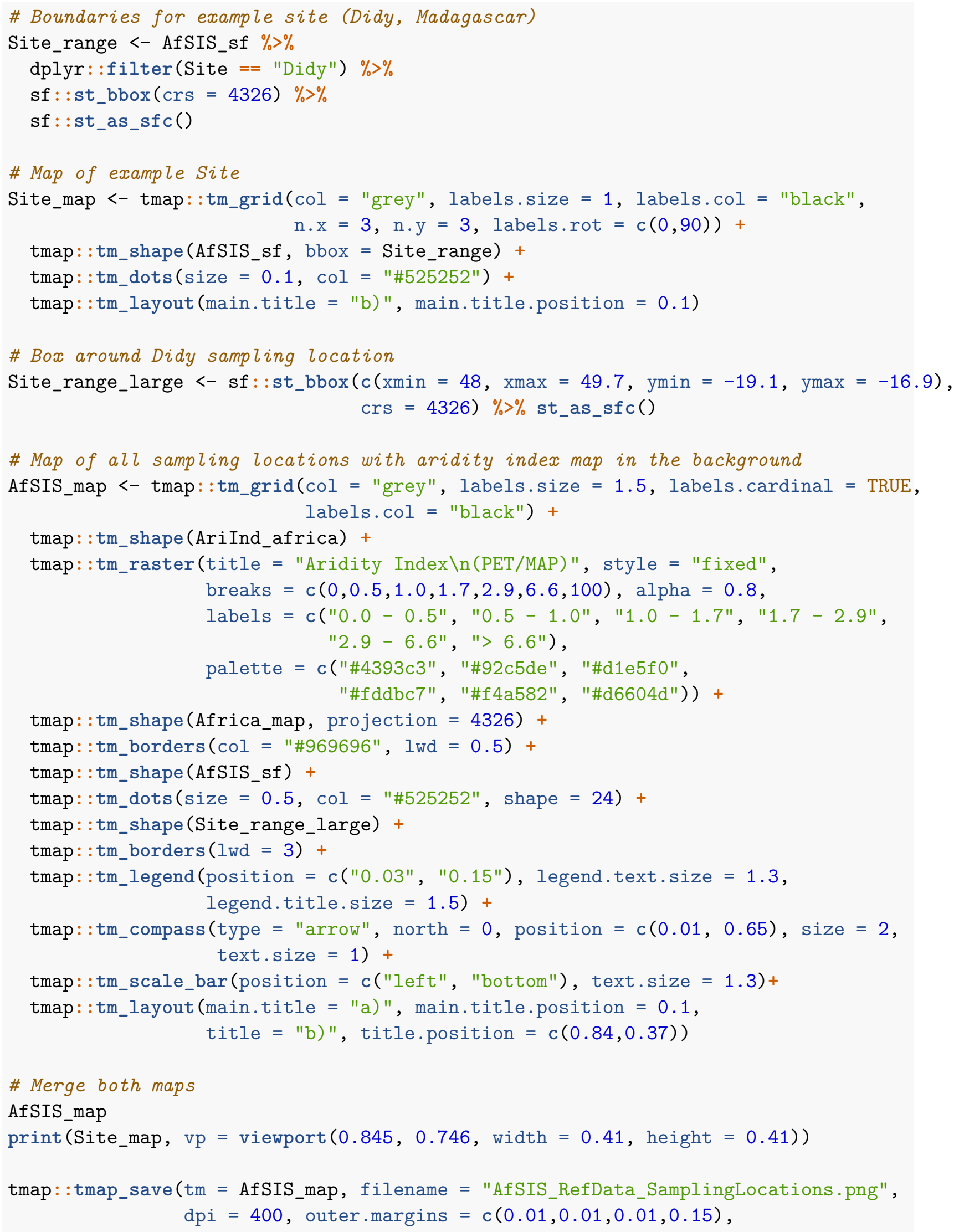




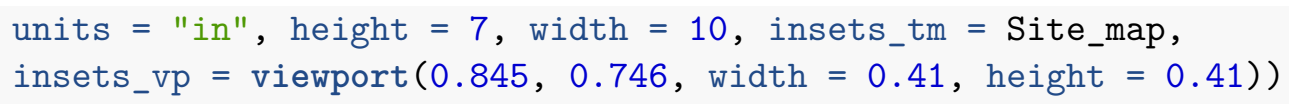

\section{Parameter distribution}

This section provides code to get an overview about the data, including summary statistics, skweness, kurtosis, histograms and shapiro-wilk tests. Some of the results are shown in Table 2 in the manuscript. In addition, it also contains the code for all the plots in the manuscript that show raw data (Figure 2, Figure 5, Figure A1 and Figure A4).

\subsection{Data distribution including skewness and kurtosis}

Table 1 in the manuscript.

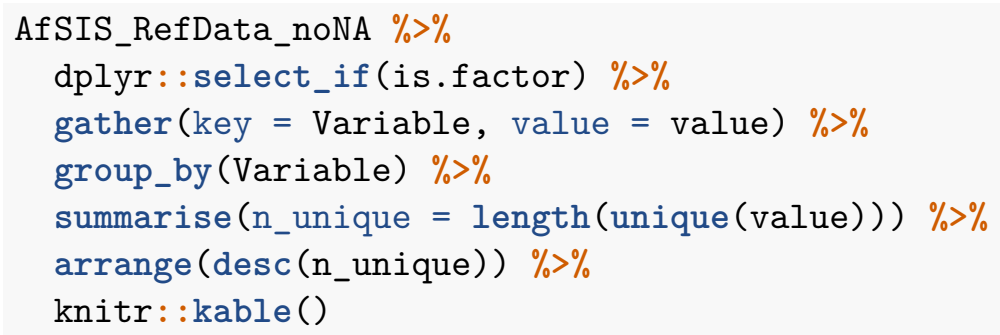

\begin{tabular}{lr}
\hline Variable & n_unique \\
\hline Site & 45 \\
Country & 17 \\
Cluster & 16 \\
Profile & 15 \\
LandCover & 4 \\
pH_Class & 4 \\
Region & 3 \\
wet_month_Class & 3 \\
ClA_Class & 2 \\
Depth & 2 \\
\hline
\end{tabular}

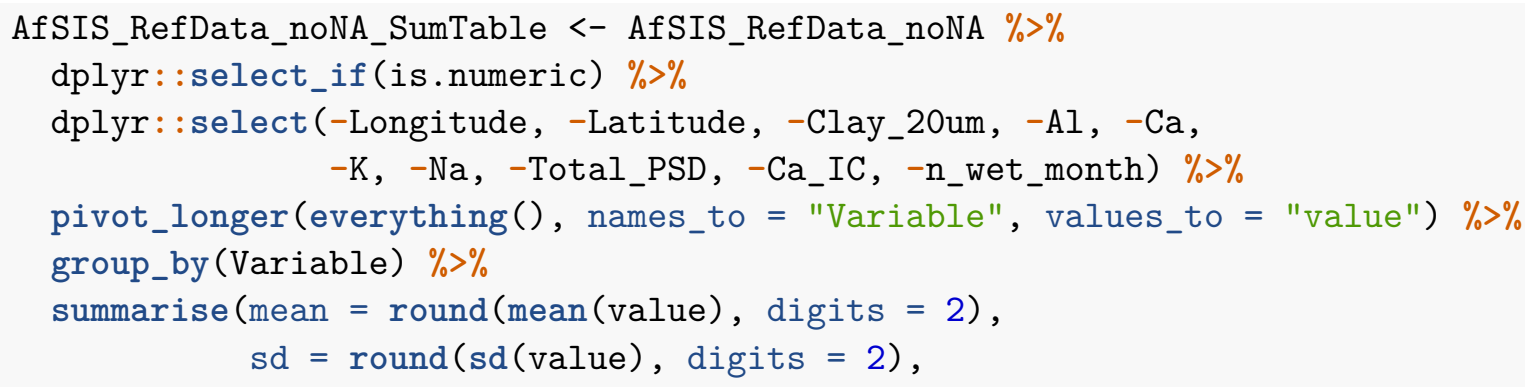




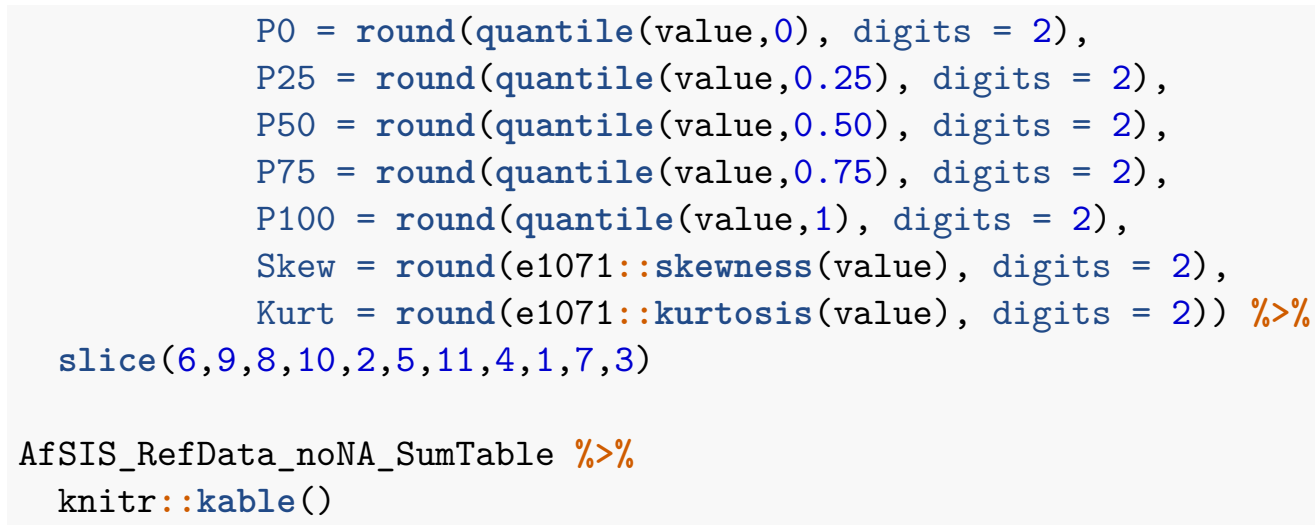

\begin{tabular}{lrrrrrrrrr}
\hline Variable & mean & sd & $\mathrm{P} 0$ & $\mathrm{P} 25$ & $\mathrm{P} 50$ & $\mathrm{P} 75$ & $\mathrm{P} 100$ & Skew & Kurt \\
\hline CORG & 1.84 & 1.51 & 0.07 & 0.65 & 1.42 & 2.54 & 9.19 & 1.42 & 2.23 \\
MAT & 21.67 & 3.24 & 13.74 & 19.80 & 21.52 & 22.95 & 29.82 & 0.17 & -0.12 \\
MAP & 1070.35 & 487.21 & 255.00 & 648.00 & 1057.00 & 1432.00 & 2708.00 & 0.29 & -0.63 \\
PET & 1810.33 & 310.46 & 1350.00 & 1571.00 & 1759.00 & 1933.00 & 2949.00 & 1.19 & 1.96 \\
Ariditylndex & 2.35 & 1.73 & 0.71 & 1.20 & 1.54 & 3.16 & 9.54 & 1.46 & 1.31 \\
Clay_8um & 55.42 & 22.64 & 0.12 & 37.70 & 57.92 & 74.73 & 100.00 & -0.26 & -1.00 \\
pH & 6.28 & 1.31 & 3.89 & 5.22 & 6.10 & 7.52 & 9.92 & 0.27 & -1.11 \\
ClA & 87.74 & 9.30 & 10.34 & 81.70 & 88.09 & 95.98 & 99.91 & -1.04 & 3.88 \\
Alox & 0.28 & 0.36 & 0.01 & 0.12 & 0.20 & 0.29 & 3.71 & 4.52 & 25.29 \\
Feox & 0.38 & 0.56 & 0.01 & 0.10 & 0.21 & 0.40 & 4.46 & 3.60 & 14.96 \\
Caex & 10.29 & 11.01 & 0.03 & 1.34 & 5.86 & 16.49 & 75.66 & 1.28 & 1.32 \\
\hline
\end{tabular}

\#Table as shown in the manuscript

AfSIS_RefData_noNA_SumTable_gt <-

AfSIS_RefData_noNA_SumTable $\%>\%$

gt()

\# gtsave(AfSIS_RefData_noNA_SumTable_gt,

\# filename $=$ "AfSIS_RefData_noNA_SumTable.rtf")

\section{Shapiro-Wilk-Test:}

Normal distribution: $p>0.05$

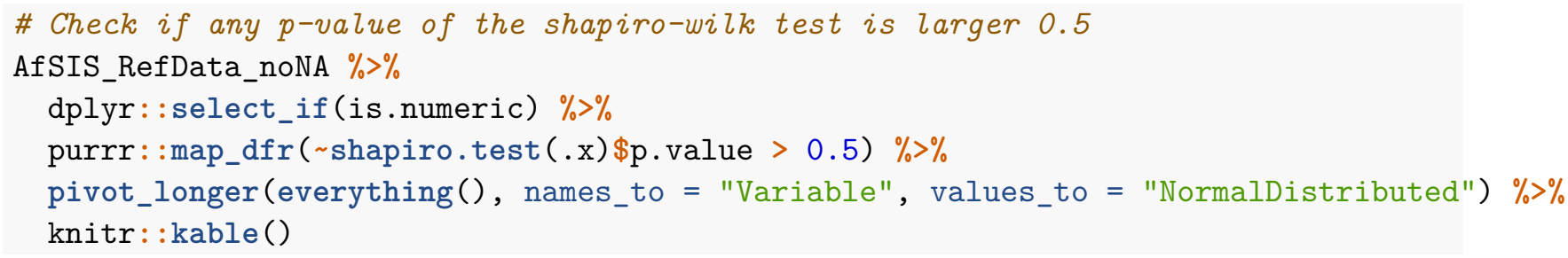




\begin{tabular}{ll}
\hline Variable & NormalDistributed \\
\hline Longitude & FALSE \\
Latitude & FALSE \\
Clay_8um & FALSE \\
Clay_20um & FALSE \\
CORG & FALSE \\
pH & FALSE \\
Alox & FALSE \\
Feox & FALSE \\
Caex & FALSE \\
Al & FALSE \\
Ca & FALSE \\
K & FALSE \\
Na & FALSE \\
Total_PSD & FALSE \\
MAT & FALSE \\
MAP & FALSE \\
PET & FALSE \\
Ariditylndex & FALSE \\
Ca_IC & FALSE \\
CIA & FALSE \\
n_wet_month & FALSE \\
\hline
\end{tabular}

Histograms:
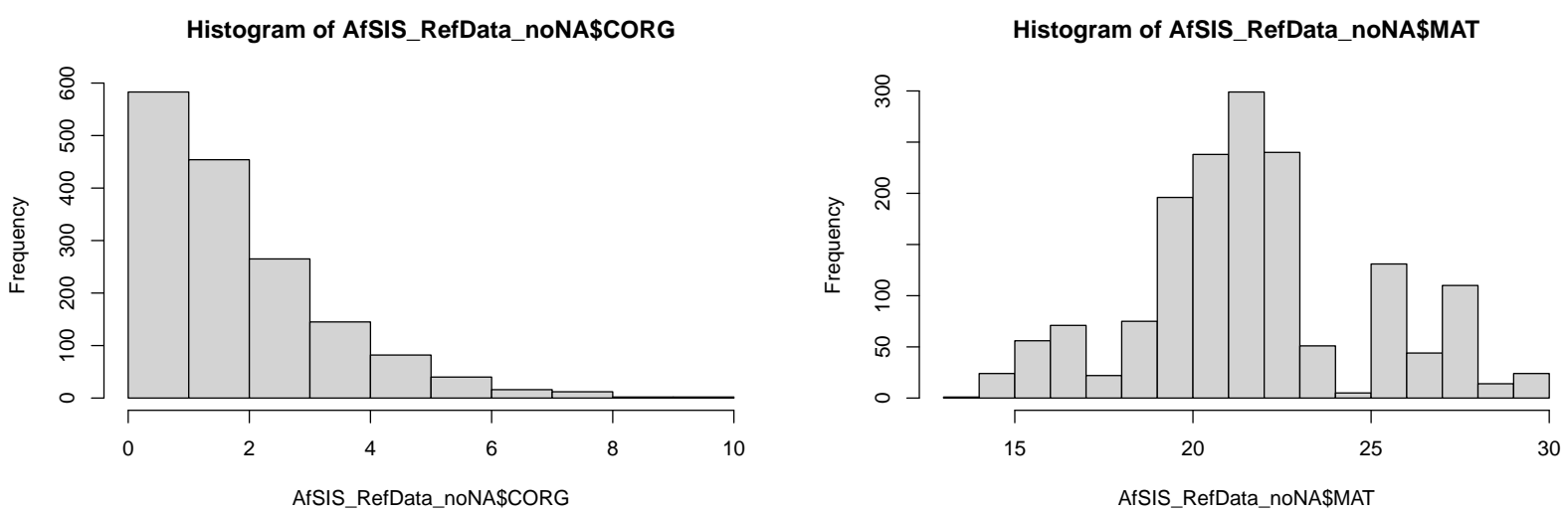


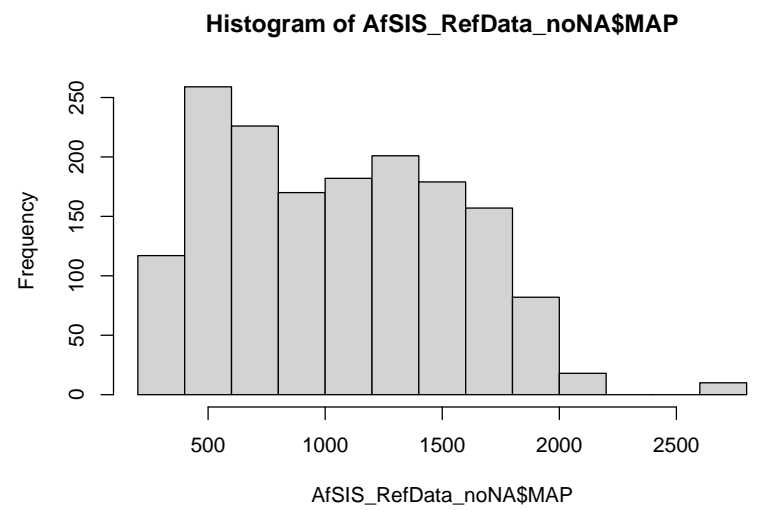

Histogram of AfSIS_RefData_noNA\$AridityIndex

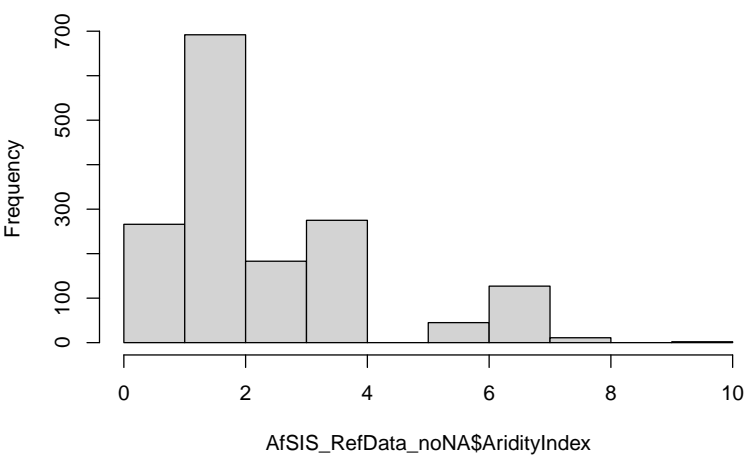

Histogram of AfSIS_RefData_noNA\$Alox

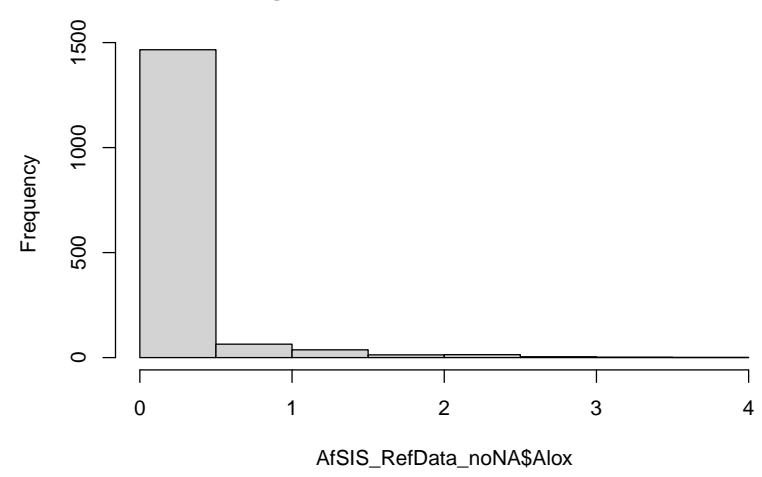

Histogram of AfSIS_RefData_noNA\$PET

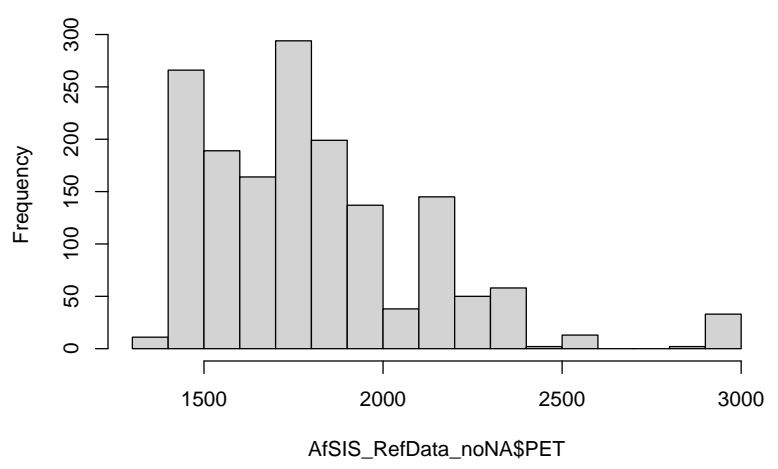

Histogram of AfSIS_RefData_noNA\$Clay_8um

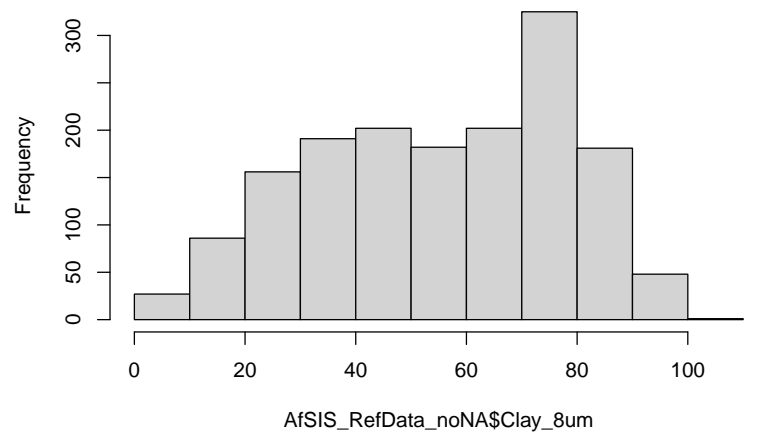

Histogram of AfSIS_RefData_noNA\$Feox

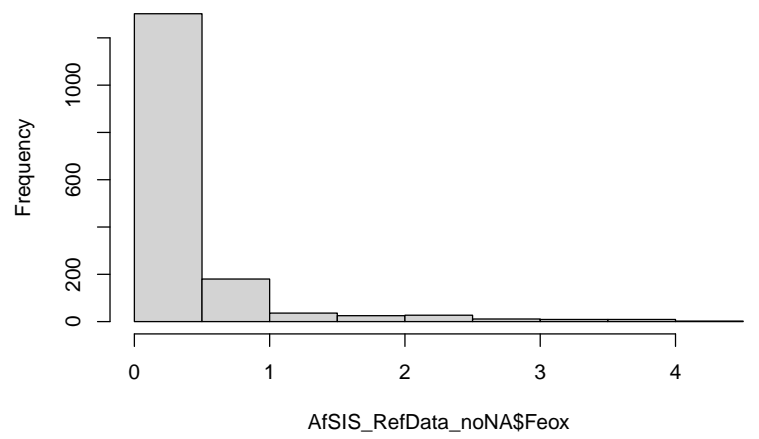



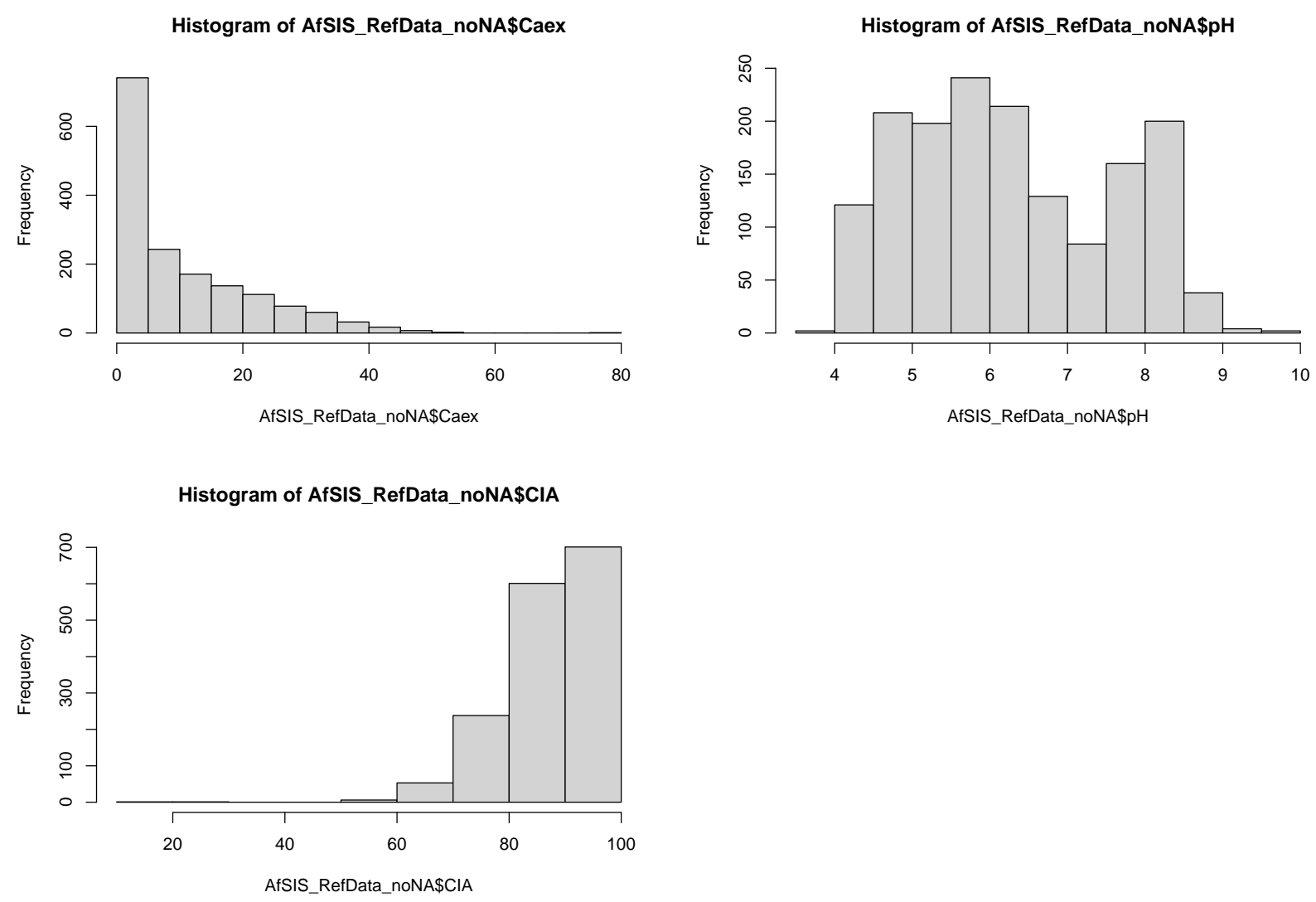

Based on Skweness, Kurtosis, Shapiro-Wilk-Test and Histograms none of the variables are normal distributed.

\subsection{Plotting}

\section{Land cover and clay content vs CORG by depth:}

Figure 2 in the manuscript and Figure $A 1$ in the supplement.

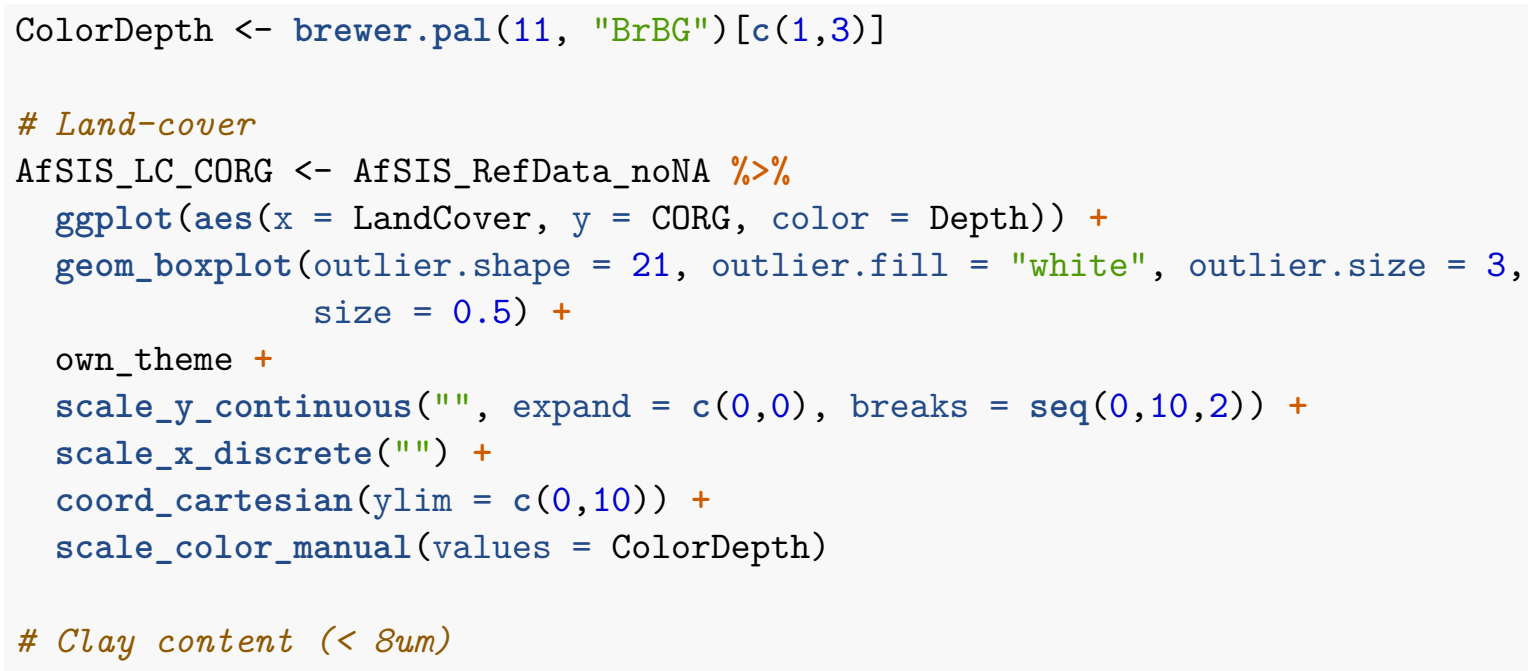




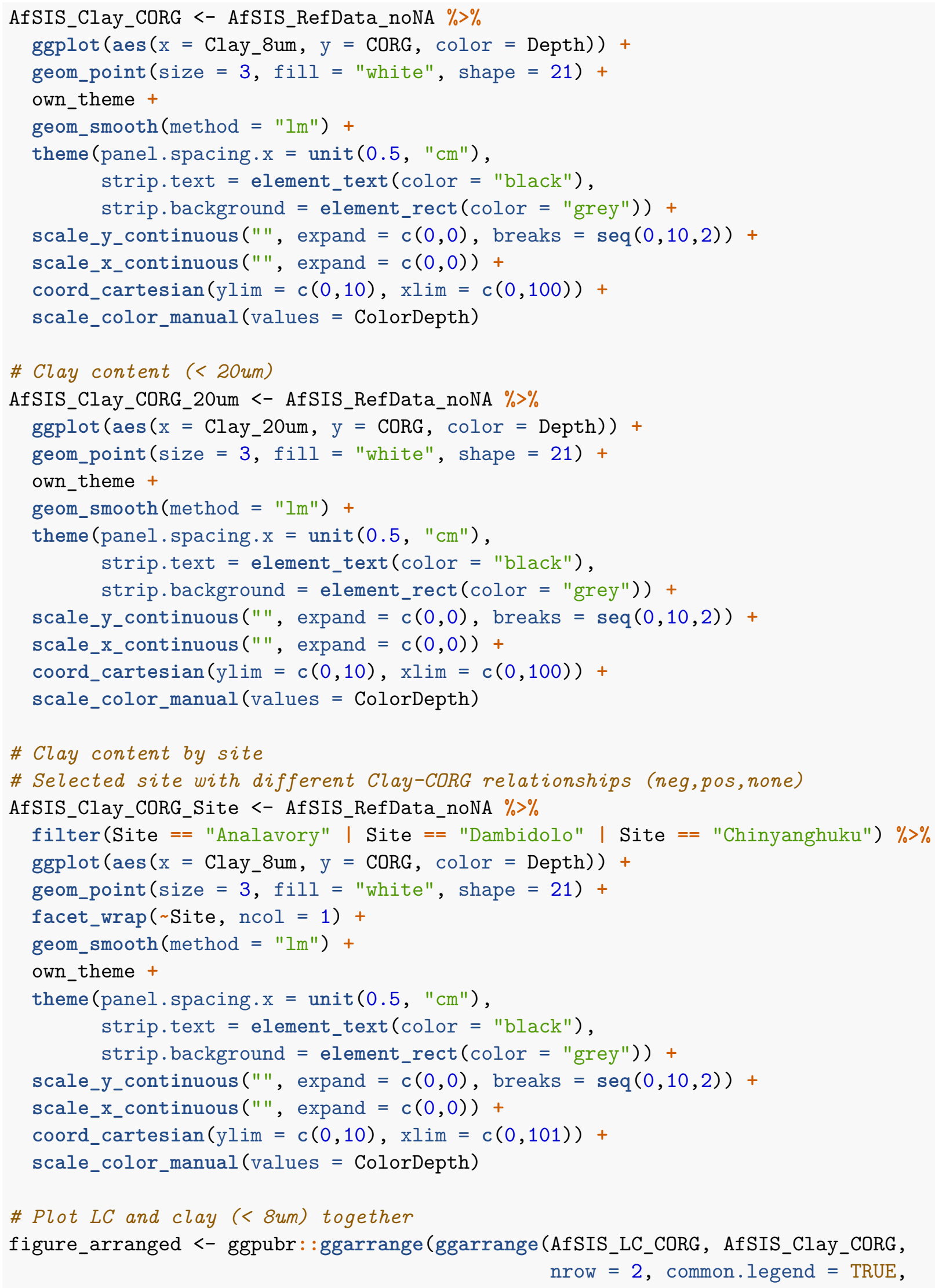




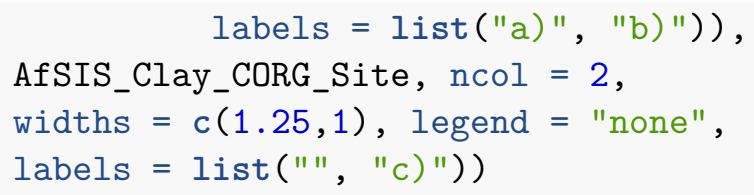

ggpubr: : annotate_figure(figure_arranged,

left $=$ text_grob $("$ SOC $[w t-\%] "$, color $=$ "black", rot $=90$, size $=14)$, bottom = text_grob("Clay + fine silt content [\%]", color $=$ "black", size $=14$, vjust $=-1$ ))

ggsave("AfSIS_RefData_Clay_LC_SOC_Depth.jpeg", height $=5$, width $=7$, dpi $=300$ )

\# Plot clay (< 8um) and clay (<20um) together

figure_arranged_clay <- ggarrange(AfSIS_Clay_CORG, AfSIS_Clay_CORG_20um, common.legend $=$ TRUE, label. $\mathrm{x}=0.051$, label. $\mathrm{y}=0.97$, labels $=c(" a)<8 \mu \mathrm{m} ", " b)<20 \mu \mathrm{m} "))$

annotate_figure(figure_arranged_clay, left $=$ text_grob("SOC $[\mathrm{wt}-\%] "$, color $=$ "black", rot $=90$, size $=12)$, bottom = text_grob("Clay + silt content $[\%] "$, color $=$ "black", size $=12$, vjust $=-1$ ))

ggsave("AfSIS_RefData_Clay_SOC_Depth.jpeg", height $=4$, width $=6$, dpi $=300$ )

\section{Clay content vs CORG by site:}

Figure $A 4$ in the supplement of the manuscript.

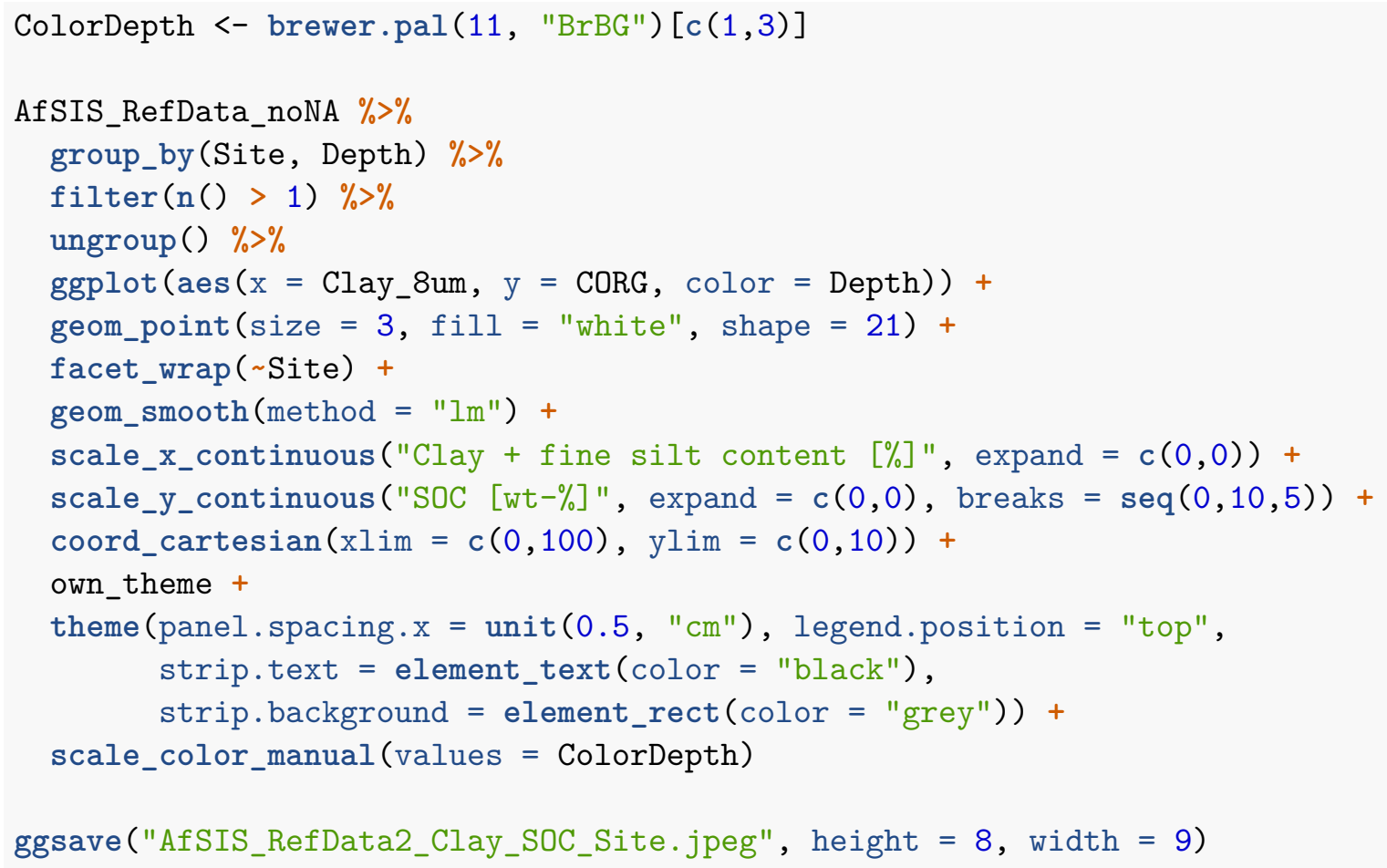




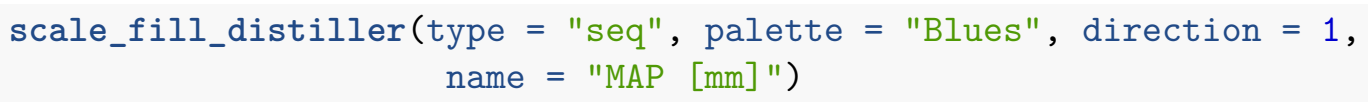

$\mathrm{Ca}_{\mathrm{ex}}$ vs CORG by pH groups:

Figure $5 a$ in the manuscript.

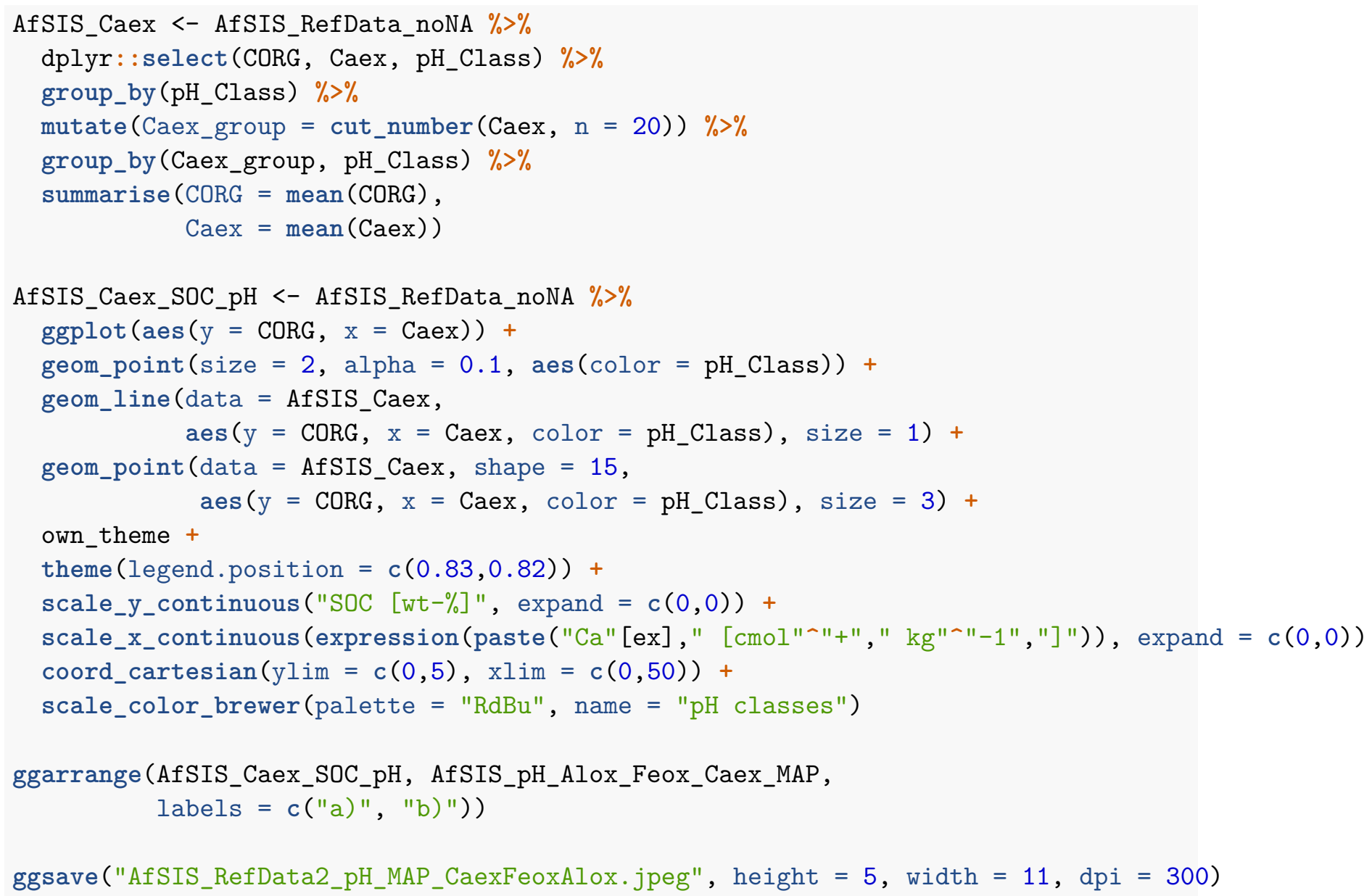

\section{Linear mixed-effects models}

This chapter contains all the code related to linear mixed-effects models, including normalization and standardization of the data, as well as all models for the entire dataset and for all the subgroups (depth, $\mathrm{pH}_{\mathrm{H} 20}$, seasonality, $\mathrm{ClA}$, land cover).

\subsection{Entire dataset models}

This section is dealing with the linear mixed-effects models for the entire dataset $(n=1,601)$. The results can be found in Figure 3 and Table B3 and B4 in the supplement of the manuscript. 


\subsubsection{All samples}

Normalize and standardize data; merge $\mathrm{Fe}_{\mathrm{ox}}$ and $\mathrm{Al}_{\mathrm{ox}}$ :

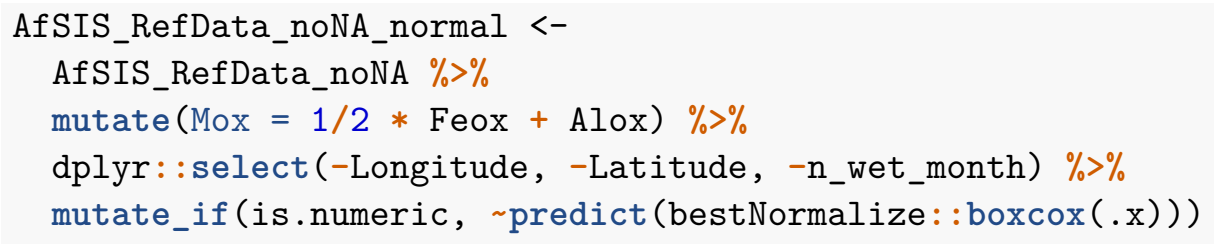

Build model (order of predictors based on a-priori knowledge):

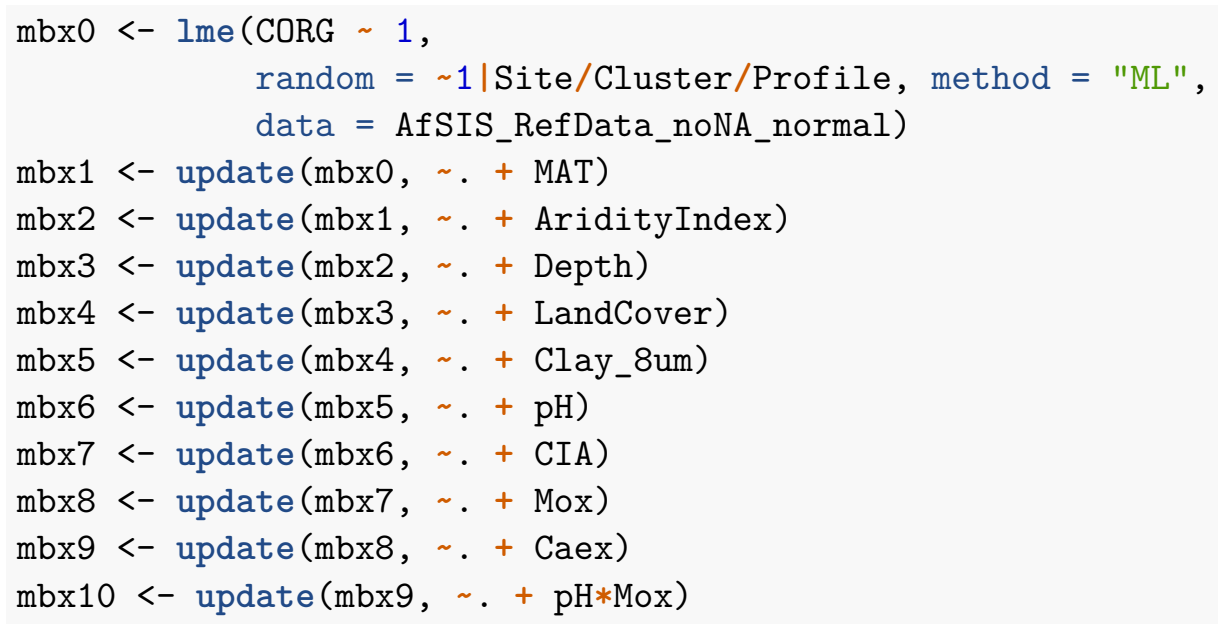

Summary output and diagnostic plots: 
summary $(\operatorname{mbx} 10)$

\#\# Linear mixed-effects model fit by maximum likelihood

\#\# Data: AfSIS_RefData_noNA_normal

\#\# AIC BIC logLik

\#\# $\quad 1599.155 \quad 1690.587 \quad-782.5773$

\#\#

\#\# Random effects:

\#\# Formula: 1 | Site

\#\# (Intercept)

\#\# StdDev: 0.3333004

\#\#

\#\# Formula: $\sim 1$ | Cluster $\%$ in $\%$ Site

\#\# (Intercept)

\#\# StdDev: 0.2256502

\#\#

\#\# Formula: $~ 1$ | Profile $\%$ in $\%$ Cluster $\%$ in $\%$ Site

\#\# (Intercept) Residual

\#\# StdDev: 0.26936150 .2428774

\#\#

\#\# Fixed effects: CORG MAT + AridityIndex + Depth + LandCover + Clay_8um + pH + \#\#

Value Std.Error DF t-value p-value

$\begin{array}{llllll}\text { \#\# (Intercept) } \quad-0.1264070 & 0.06345588 & 783 & -1.992045 & 0.0467\end{array}$

\#\# MAT

$\begin{array}{lll}-0.1414200 & 0.04566240783\end{array}$

$\begin{array}{ll}-3.097076 & 0.0020\end{array}$

\#\# AridityIndex

$\begin{array}{lll}-0.4273799 & 0.05648697783\end{array}$

$\begin{array}{ll}-7.565990 & 0.0000\end{array}$

\#\# Depth.L

$\begin{array}{lllll}-0.3086967 & 0.01158359 & 343 & -26.649481\end{array}$

0.0000

\#\# LandCoverForest

$\begin{array}{lll}0.2567720 & 0.08176803 & 783\end{array}$

3.140250

0.0018

\#\# LandCoverGrassland

$\begin{array}{llll}0.0249580 & 0.04219473 & 783\end{array}$

$0.591496 \quad 0.5544$

\#\# LandCoverOther

$\begin{array}{llll}0.0832766 & 0.03573248 & 783\end{array}$

2.330557

0.0200

\#\# Clay_8um

$\begin{array}{lll}0.0068470 & 0.01701617343\end{array}$

0.402380

0.6877

\#\# $\mathrm{pH}$

$\begin{array}{llll}-0.3667555 & 0.03017725 & 343 & -12.153378\end{array}$

0.0000

\#\# CIA

$\begin{array}{llll}-0.2174211 & 0.01991854 & 343 & -10.915514\end{array}$

0.0000

\#\# Mox

$\begin{array}{lllll}0.3211101 & 0.02198865 & 343 & 14.603450\end{array}$

0.0000

\#\# Caex

$\begin{array}{lllll}0.5262726 & 0.03003944 & 343 & 17.519387\end{array}$

0.0000

\#\# $\mathrm{pH}:$ Mox

$-0.1587035$

0.01740751343

$-9.116952$

0.0000

\#\# Correlation:

\#\#

(Intr) MAT ArdtyI Dpth.L LndCvF LndCvG LndCvO Cly_8m

\#\# MAT

$-0.188$

\#\# AridityIndex

$-0.188-0.020$

\#\# Depth.L

$\begin{array}{lll}-0.001 & 0.013 & -0.045\end{array}$

\#\# LandCoverForest

$-0.188$

$0.026 \quad 0.084$

\#\# LandCoverGrassland

\#\# LandCoverOther

$-0.279$

$0.051-0.073$

0.011

\#\# Clay_8um

\#\# $\mathrm{pH}$

\#\# CIA

$\begin{array}{llll}-0.338 & 0.038 & -0.098\end{array}$

0.0010 .152

$\begin{array}{lllll}0.032 & -0.006 & -0.003 & -0.254 & -0.002\end{array}$

0.520

$\begin{array}{lllll}0.020 & -0.028 & -0.139 & -0.094 & 0.005\end{array}$

0.042

$-0.048-0.026$

$0.065-0.105 \quad 0.044$

0.067

0.048

\#\# Mox

$0.028 \quad 0.086$

0.161

$\begin{array}{lllll}0.022 & -0.012-0.031 & -0.005 & -0.263\end{array}$ 


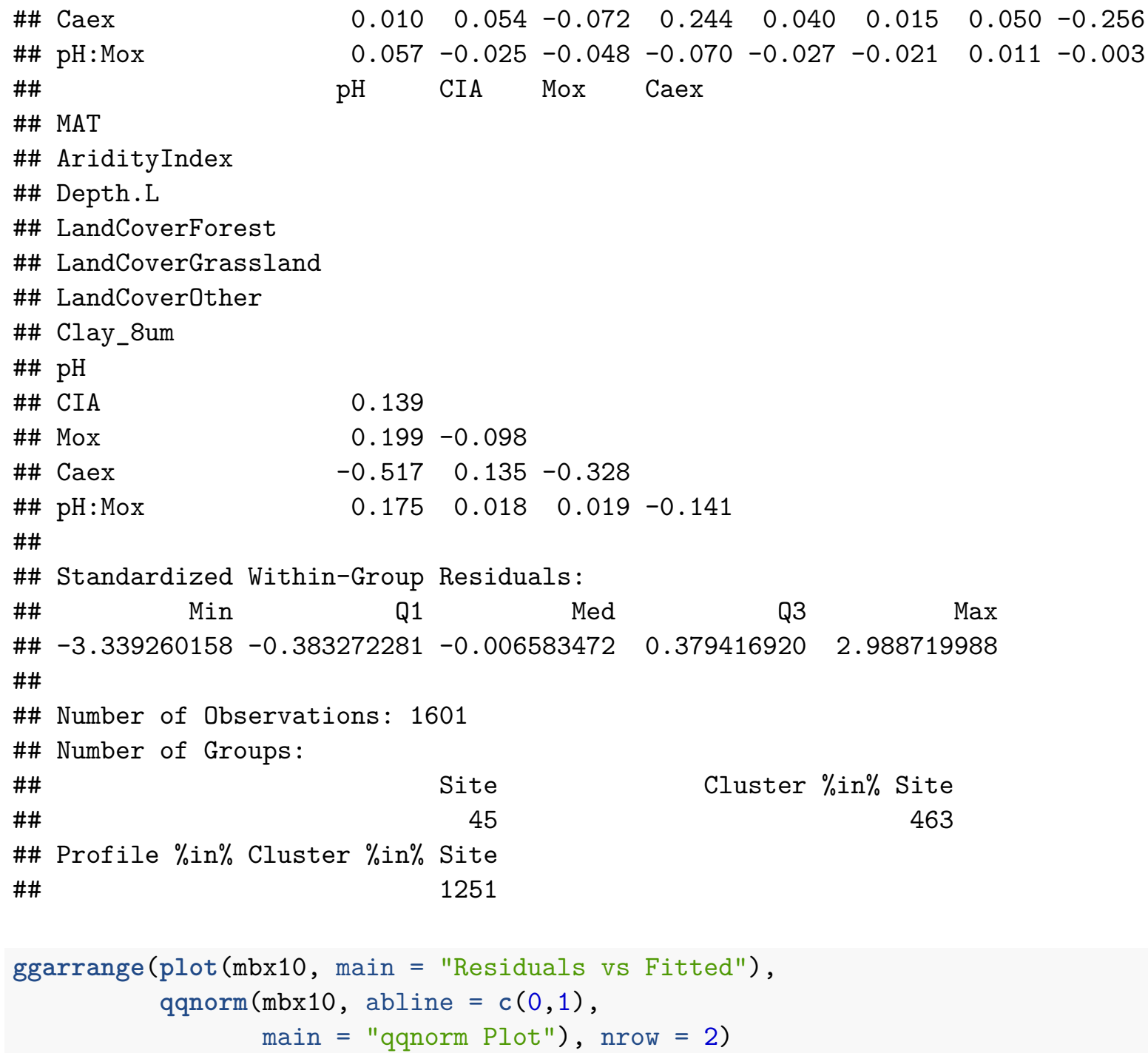



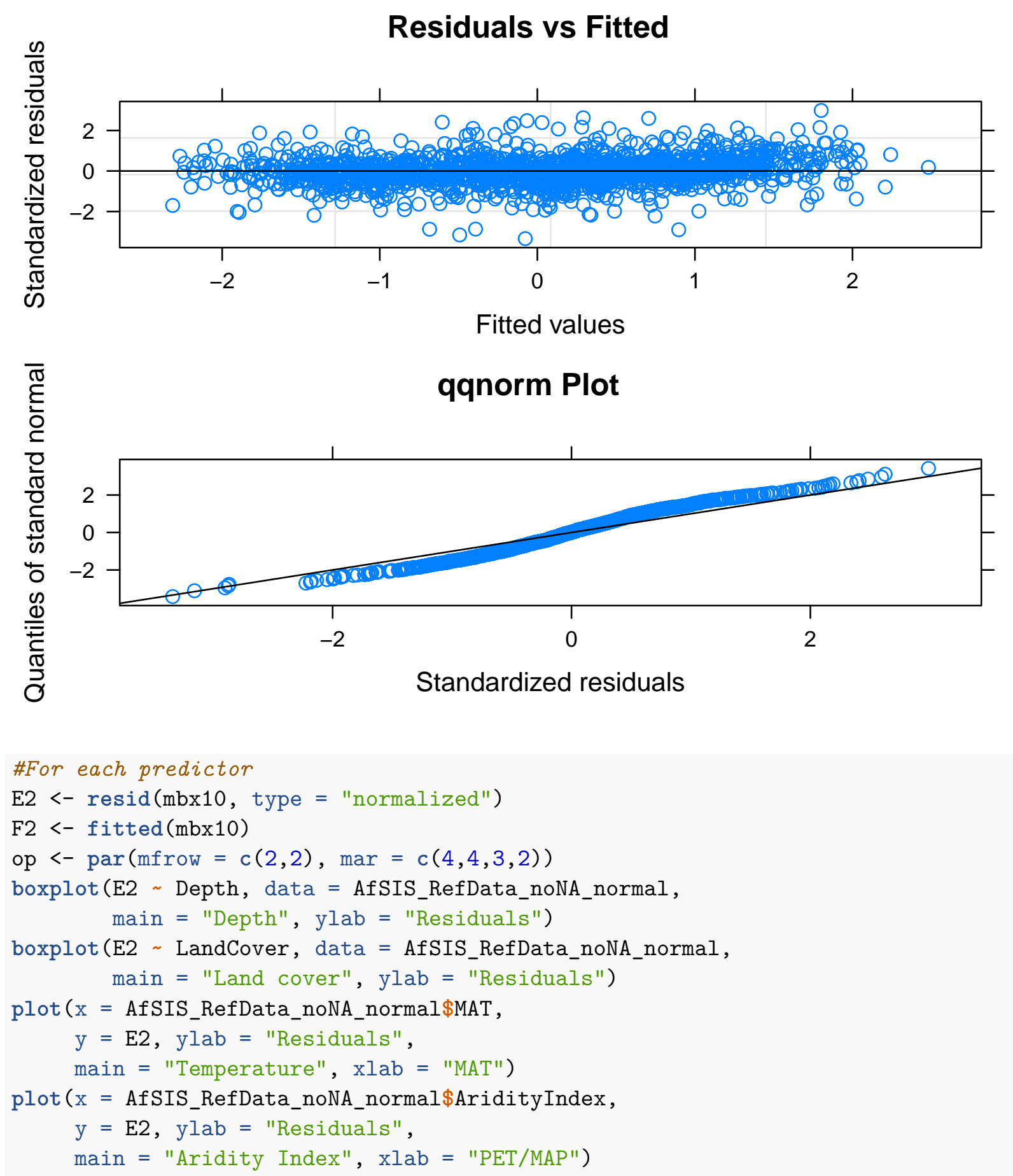
Depth

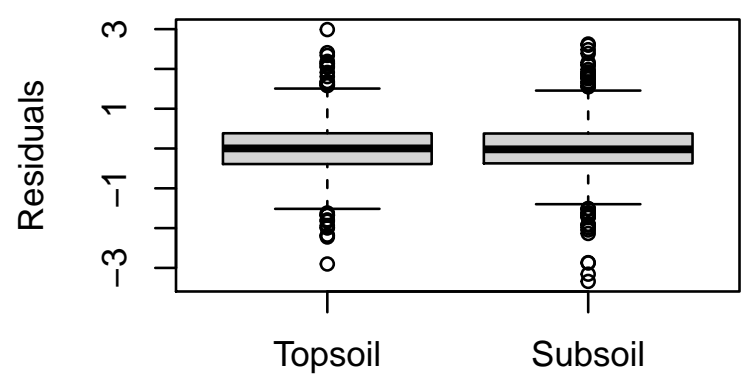

Depth

Temperature

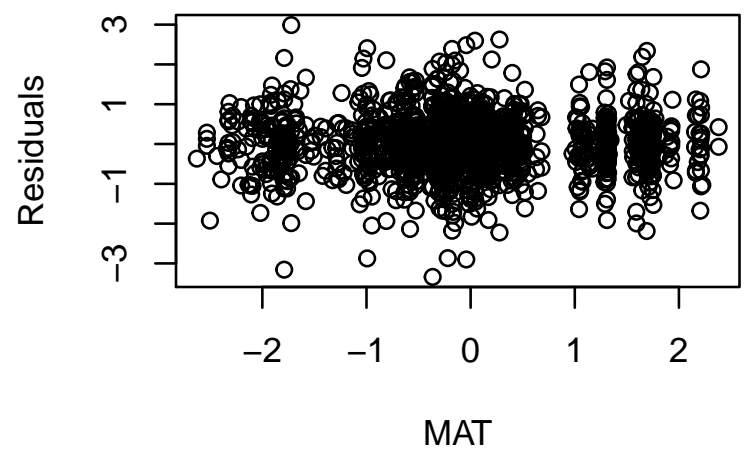

\section{Land cover}

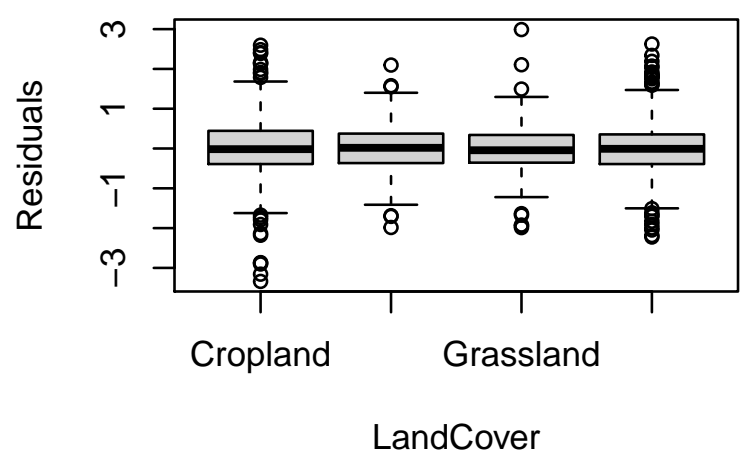

Aridity Index

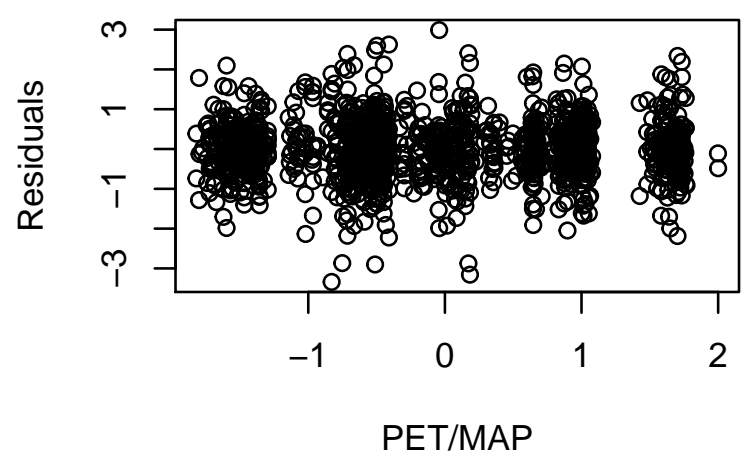

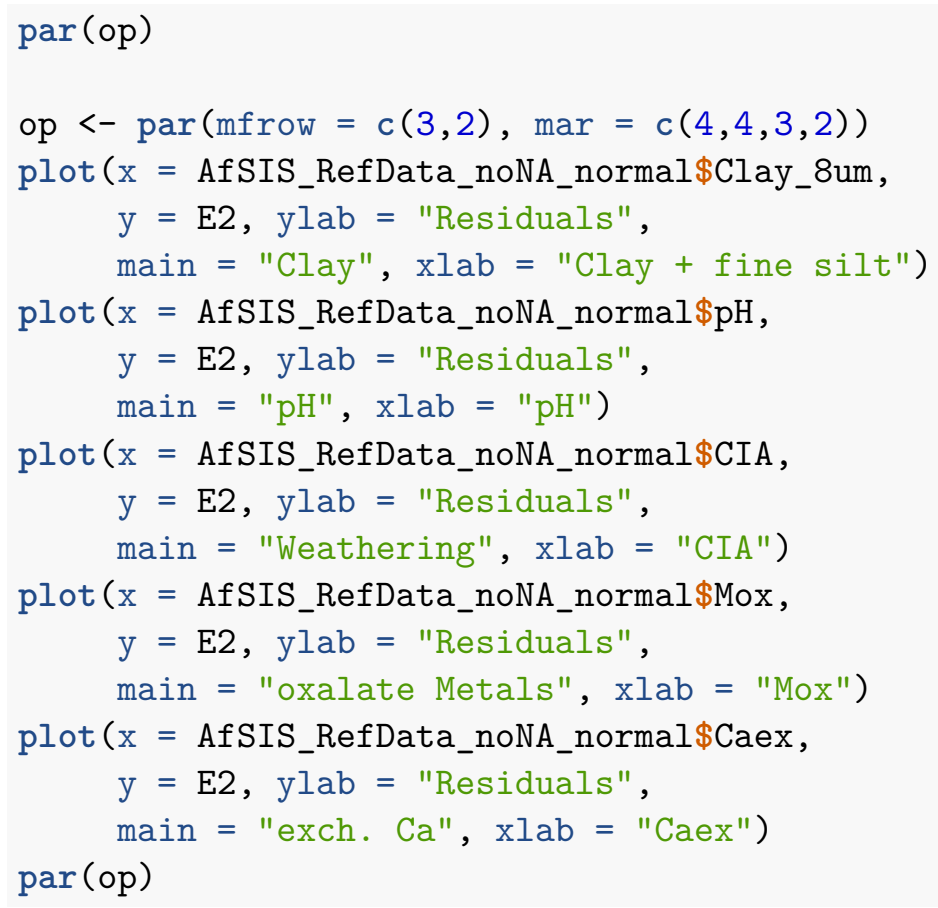


Clay

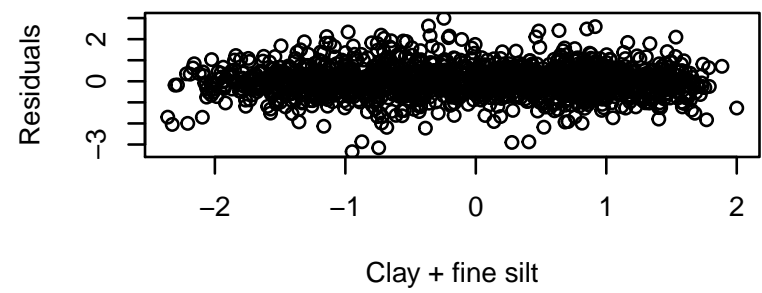

Weathering

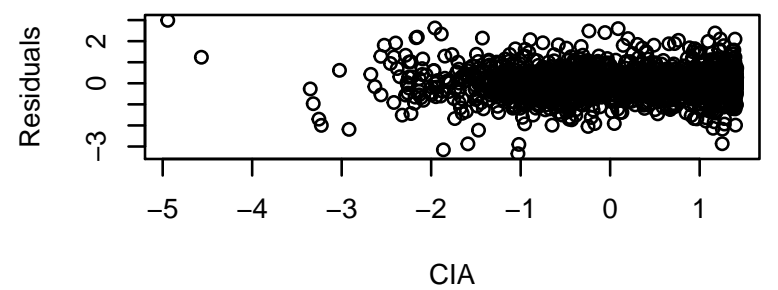

exch. Ca

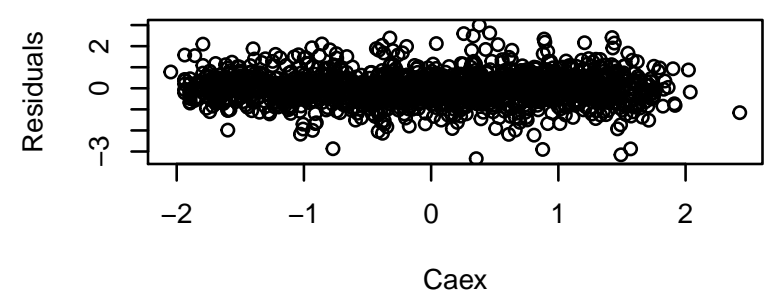

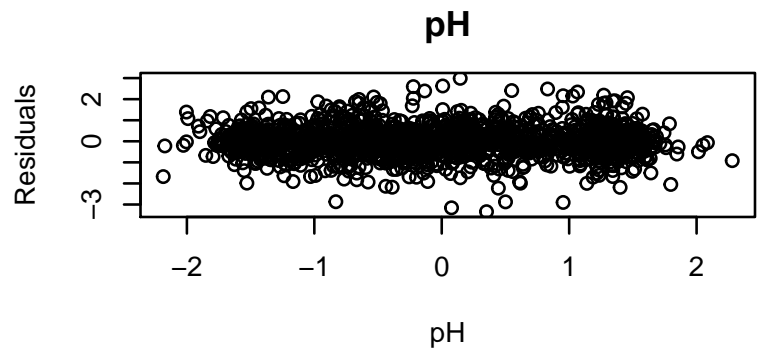

oxalate Metals

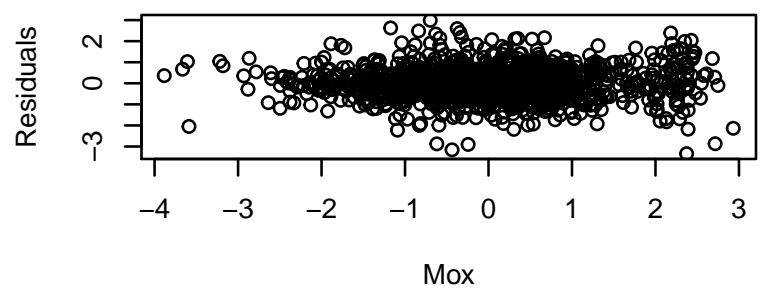

Model assumptions are met.

Anova output all models (step-wise) and $\mathrm{R}^{2}$ for full model:

ava_all <- anova (mbx0,mbx1, mbx2, mbx3, mbx4, mbx5, mbx6, mbx7, mbx8, mbx $9, m b x 10)$ ava_all

$\begin{array}{lrrrrrrrrr}\text { \#\# } & \text { Model } & \text { df } & \text { AIC } & \text { BIC } & \text { logLik } & \text { Test } & \text { L.Ratio } & \text { p-value } \\ \text { \#\# mbx0 } & 1 & 5 & 2993.221 & 3020.113 & -1491.6107 & & & & \\ \text { \#\# mbx1 } & 2 & 6 & 2968.995 & 3001.266 & -1478.4977 & 1 \text { vs } 2 & 26.2261 & <.0001 \\ \text { \#\# mbx2 } & 3 & 7 & 2932.498 & 2970.147 & -1459.2490 & 2 & \text { vs } 3 & 38.4972 & <.0001 \\ \text { \#\# mbx3 } & 4 & 8 & 2414.211 & 2457.238 & -1199.1053 & 3 & \text { vs } 4 & 520.2875 & <.0001 \\ \text { \#\# mbx4 } & 5 & 11 & 2416.057 & 2475.219 & -1197.0286 & 4 & \text { vs } 5 & 4.1534 & 0.2454 \\ \text { \#\# mbx5 } & 6 & 12 & 2340.403 & 2404.943 & -1158.2012 & 5 & \text { vs } 6 & 77.6547 & <.0001 \\ \text { \#\# mbx6 } & 7 & 13 & 2342.005 & 2411.923 & -1158.0023 & 6 \text { vs } 7 & 0.3980 & 0.5281 \\ \text { \#\# mbx7 } & 8 & 14 & 2248.878 & 2324.176 & -1110.4392 & 7 & \text { vs } 8 & 95.1260 & <.0001 \\ \text { \#\# mbx8 } & 9 & 15 & 1915.316 & 1995.992 & -942.6582 & 8 \text { vs } 9 & 335.5620 & <.0001 \\ \text { \#\# mbx9 } & 10 & 16 & 1678.088 & 1764.142 & -823.0440 & 9 & \text { vs } 10 & 239.2284 & <.0001 \\ \text { \#\# mbx10 } & 11 & 17 & 1599.155 & 1690.587 & -782.5773 & 10 \text { vs } 11 & 80.9334 & <.0001\end{array}$

\#Full model

r.squaredGLMM (mbx10) 
\#\# $\quad$ R2m $\quad$ R2c

$\begin{array}{llll}\text { \#\# }[1,] & 0.7162272 & 0.9429757\end{array}$

\subsubsection{Geochemistry model}

Build models:

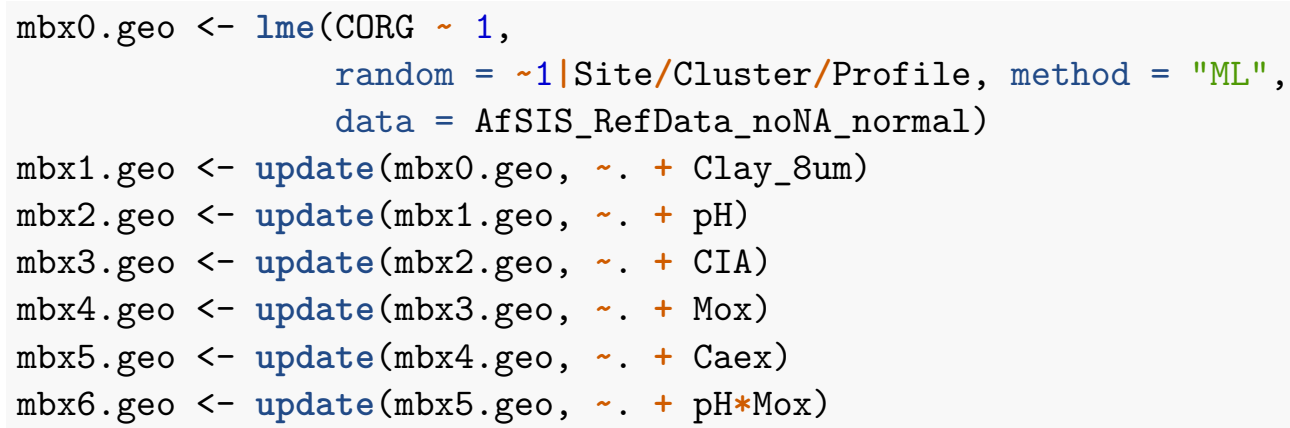

Diagnostic plots:

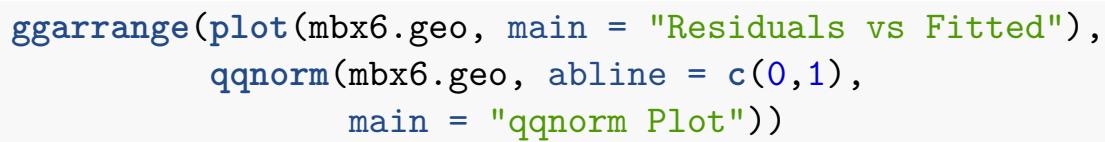

Residuals vs Fitted

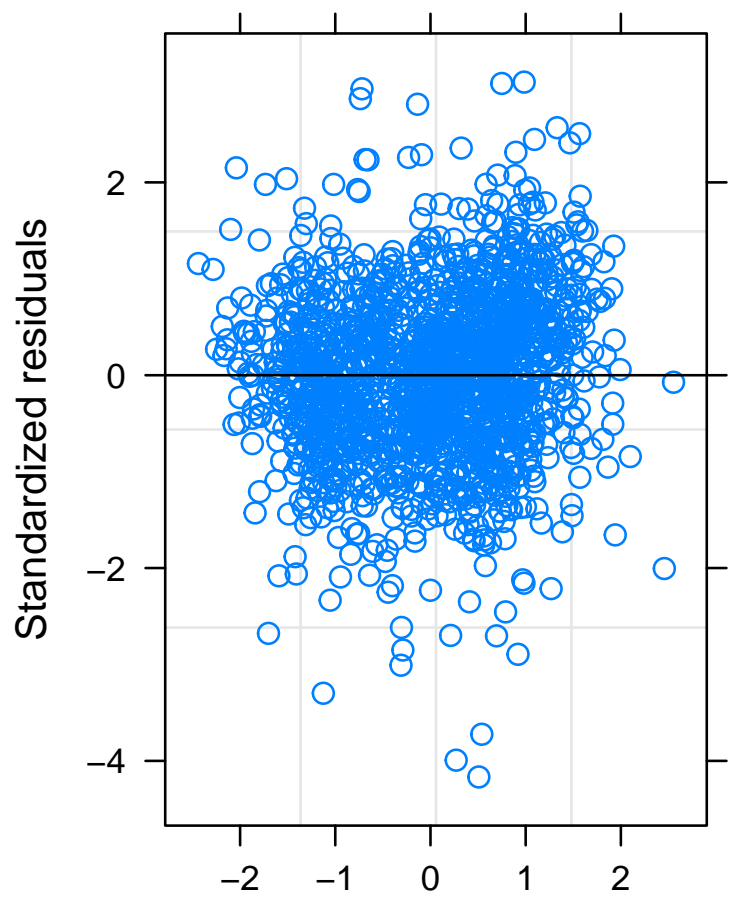

Fitted values qqnorm Plot

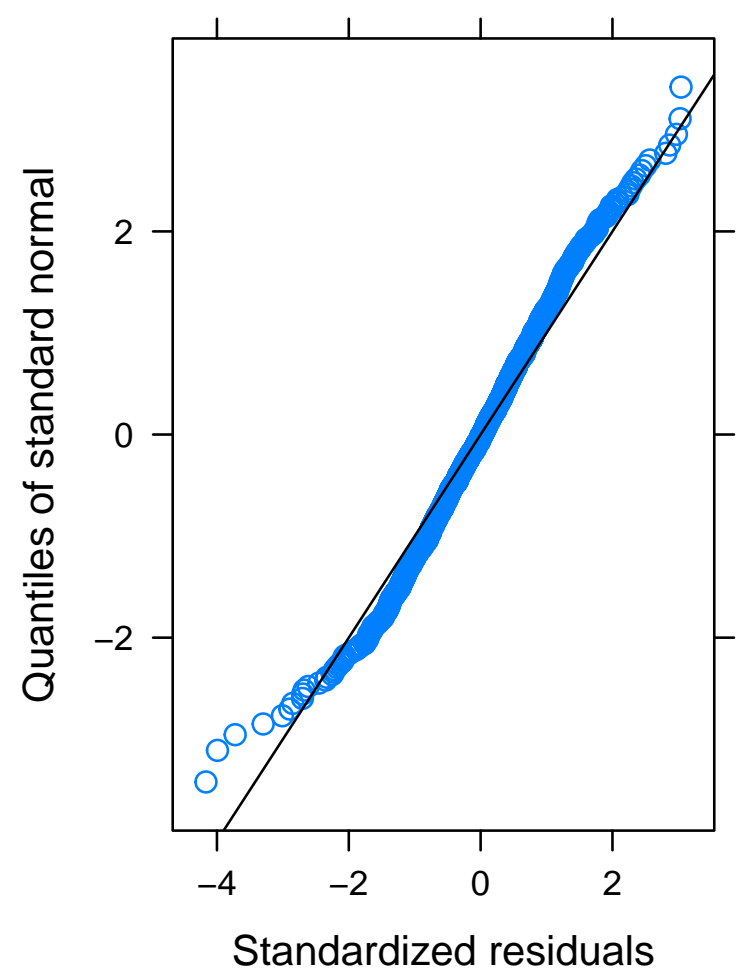


Model assumptions are met.

Anova output for all geochemistry models (step-wise):

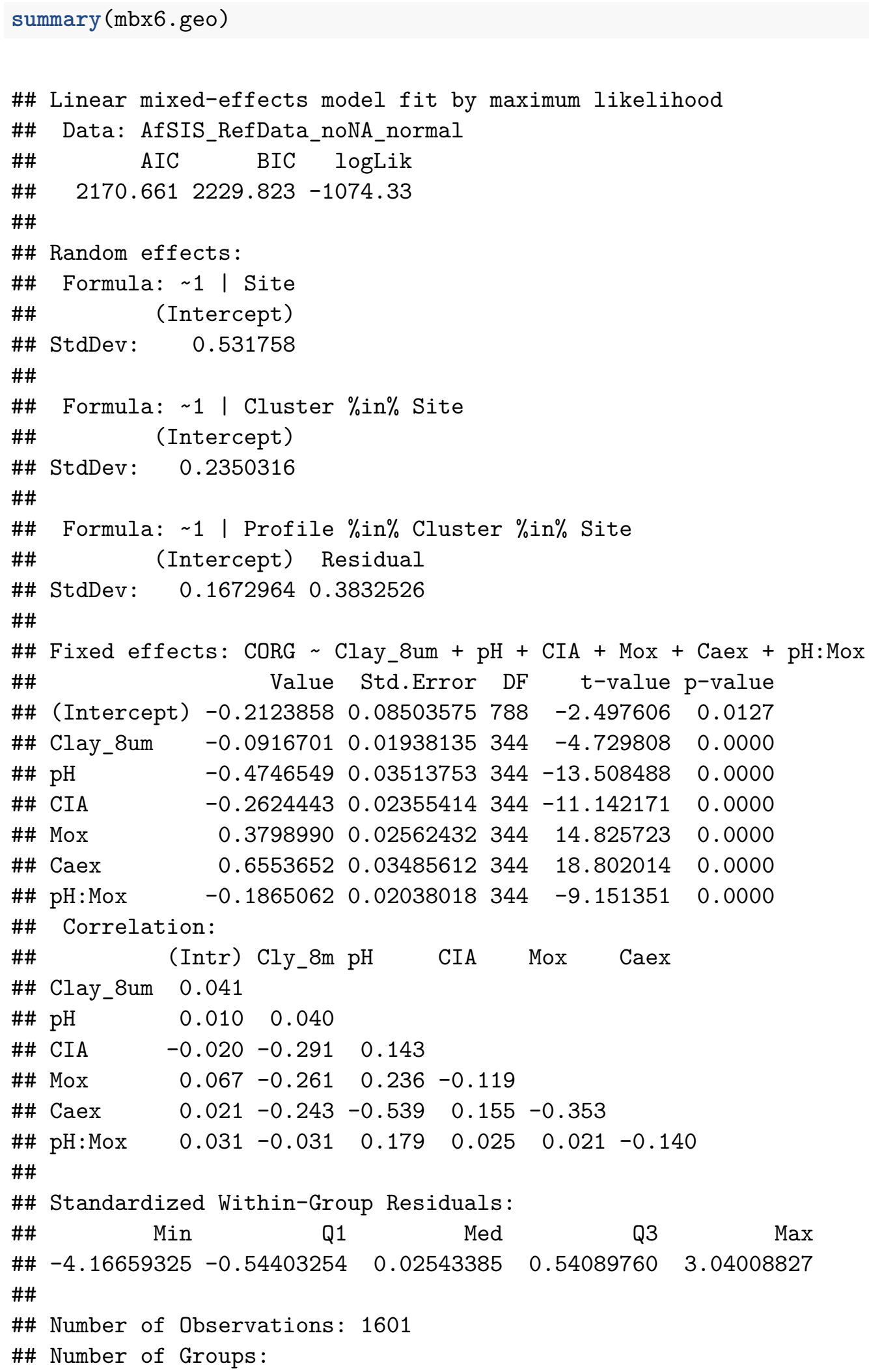




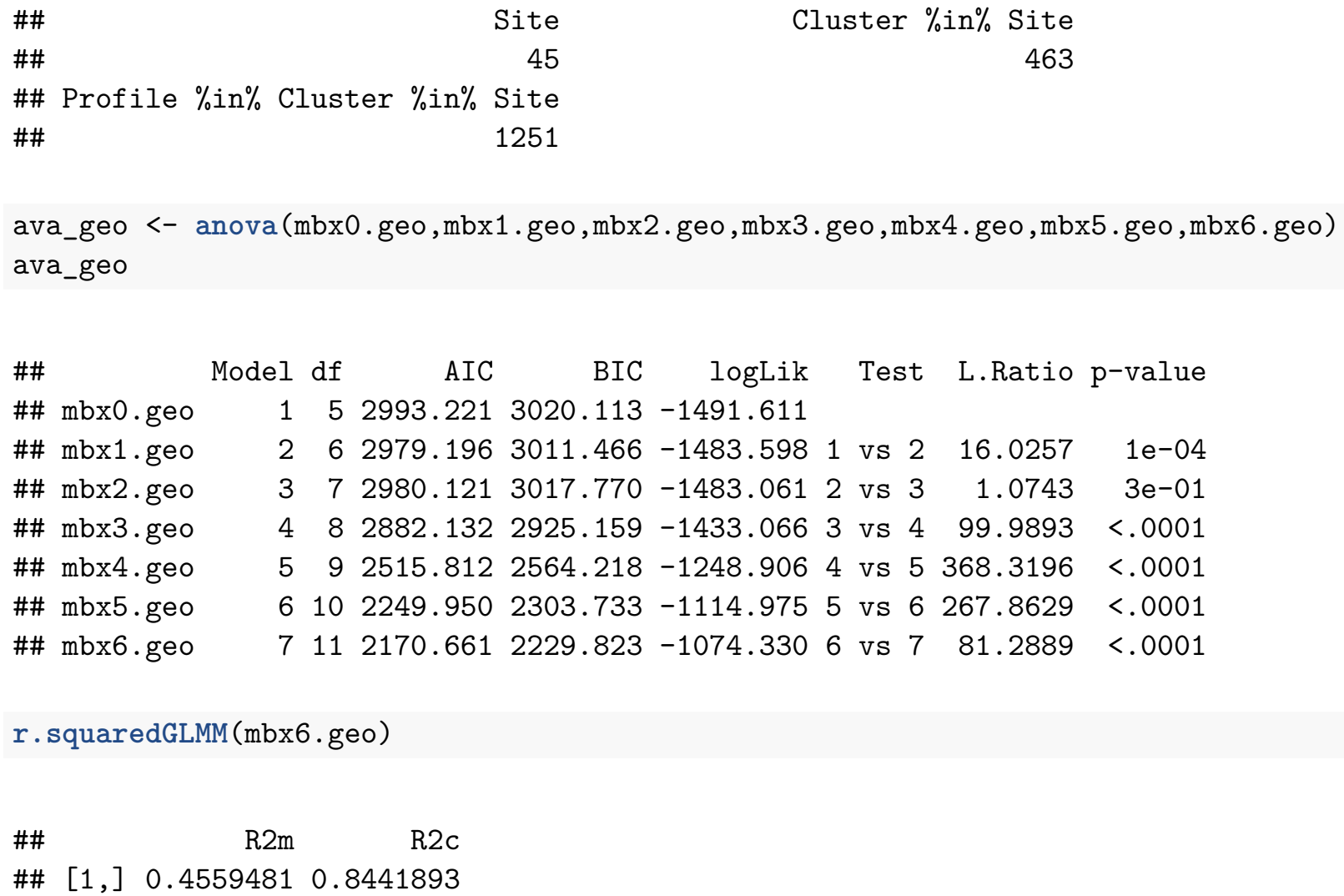

\subsubsection{Climate model}

Build models:

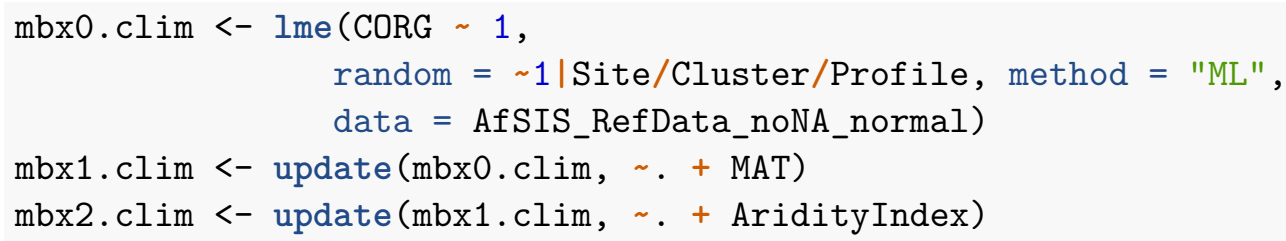

Diagnostic plots:

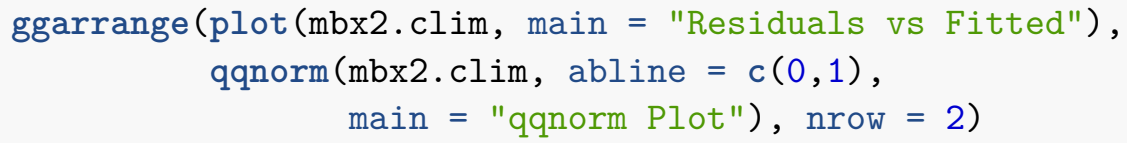




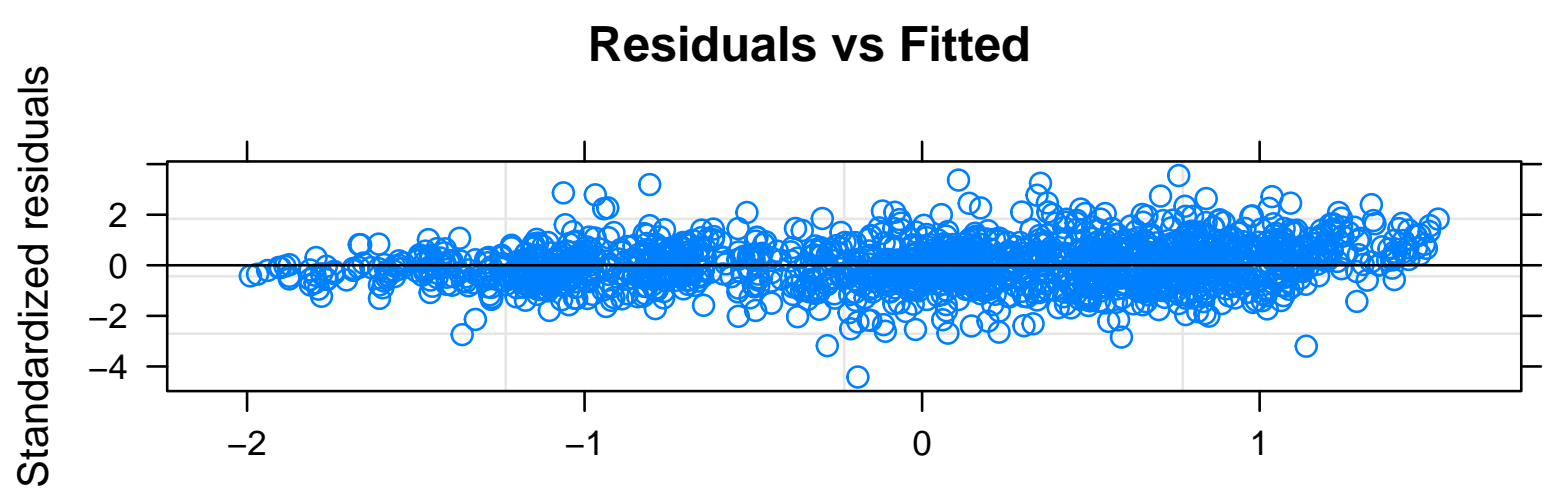

Fitted values

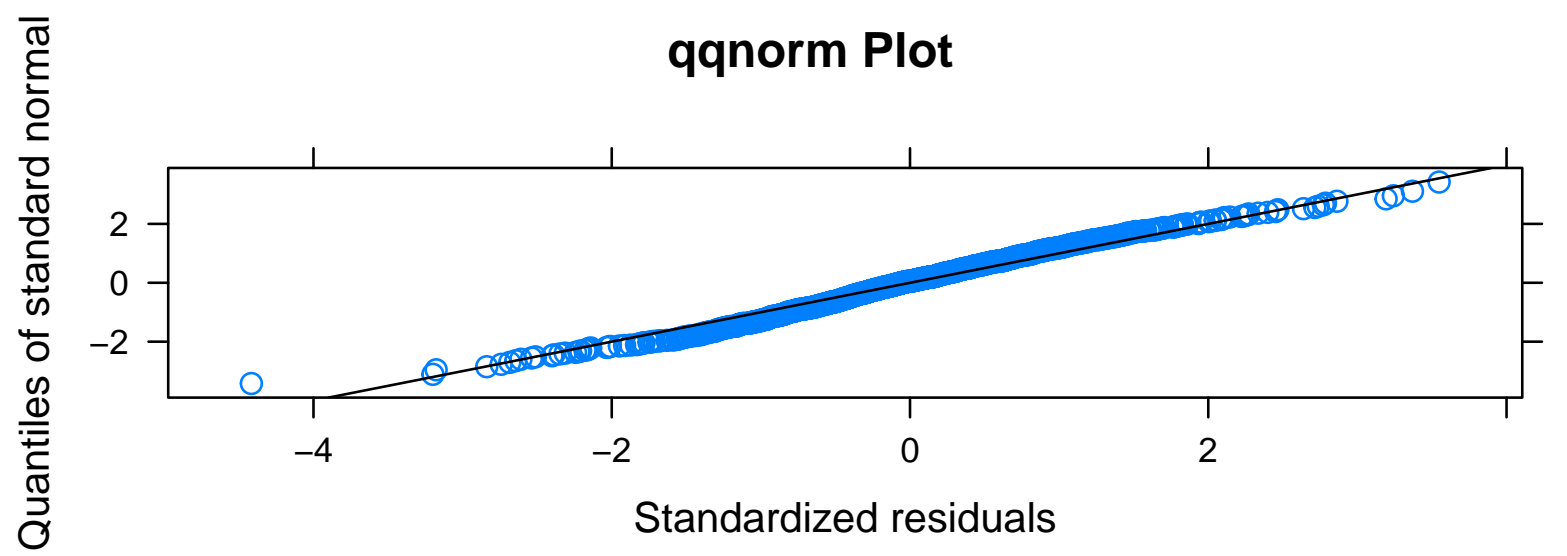

Model assumptions are met.

Anova output for all climate models (step-wise):

summary (mbx2.clim)

\#\# Linear mixed-effects model fit by maximum likelihood

\#\# Data: AfSIS_RefData_noNA_normal

\#\# AIC BIC logLik

$\begin{array}{llll}\text { \#\# } \quad 2932.498 & 2970.147 & -1459.249\end{array}$

\#\#

\#\# Random effects:

\#\# Formula: $\sim 1$ | Site

\#\# (Intercept)

\#\# StdDev: 0.5021366

\#\#

\#\# Formula: $\sim 1$ | Cluster \%in\% Site

\#\# (Intercept)

\#\# StdDev: 0.3043013

\#\#

\#\# Formula: $\sim 1$ | Profile $\%$ in $\%$ Cluster $\%$ in $\%$ Site

\#\# (Intercept) Residual

\#\# StdDev: 0.19306230 .497936 


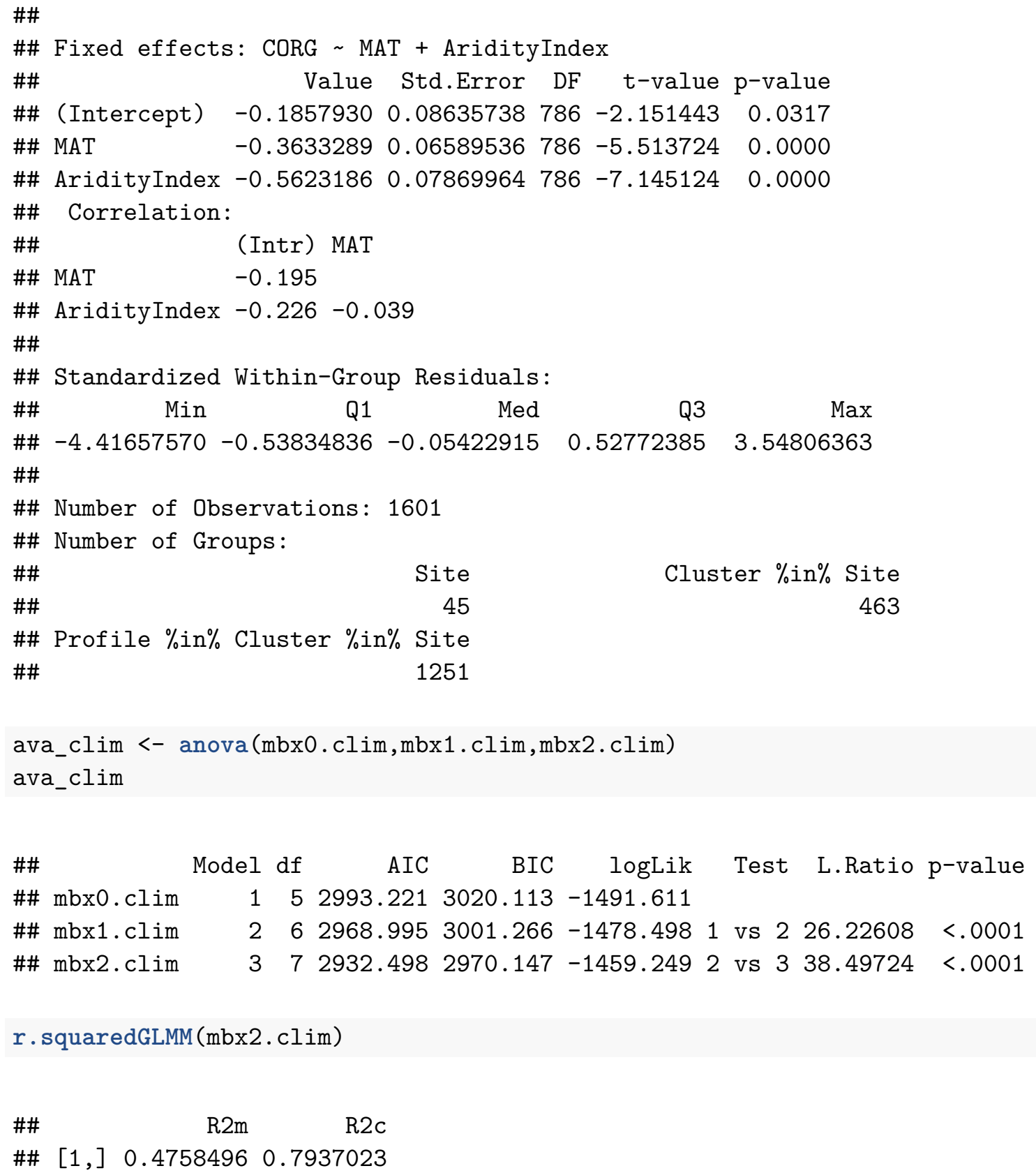

\subsubsection{Summary tables}

The summary tables can be found in Figure 3 and Table B4-B8.

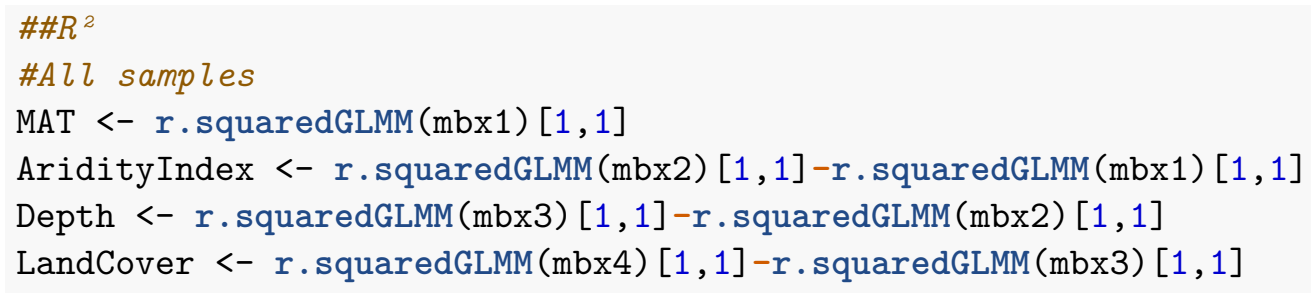




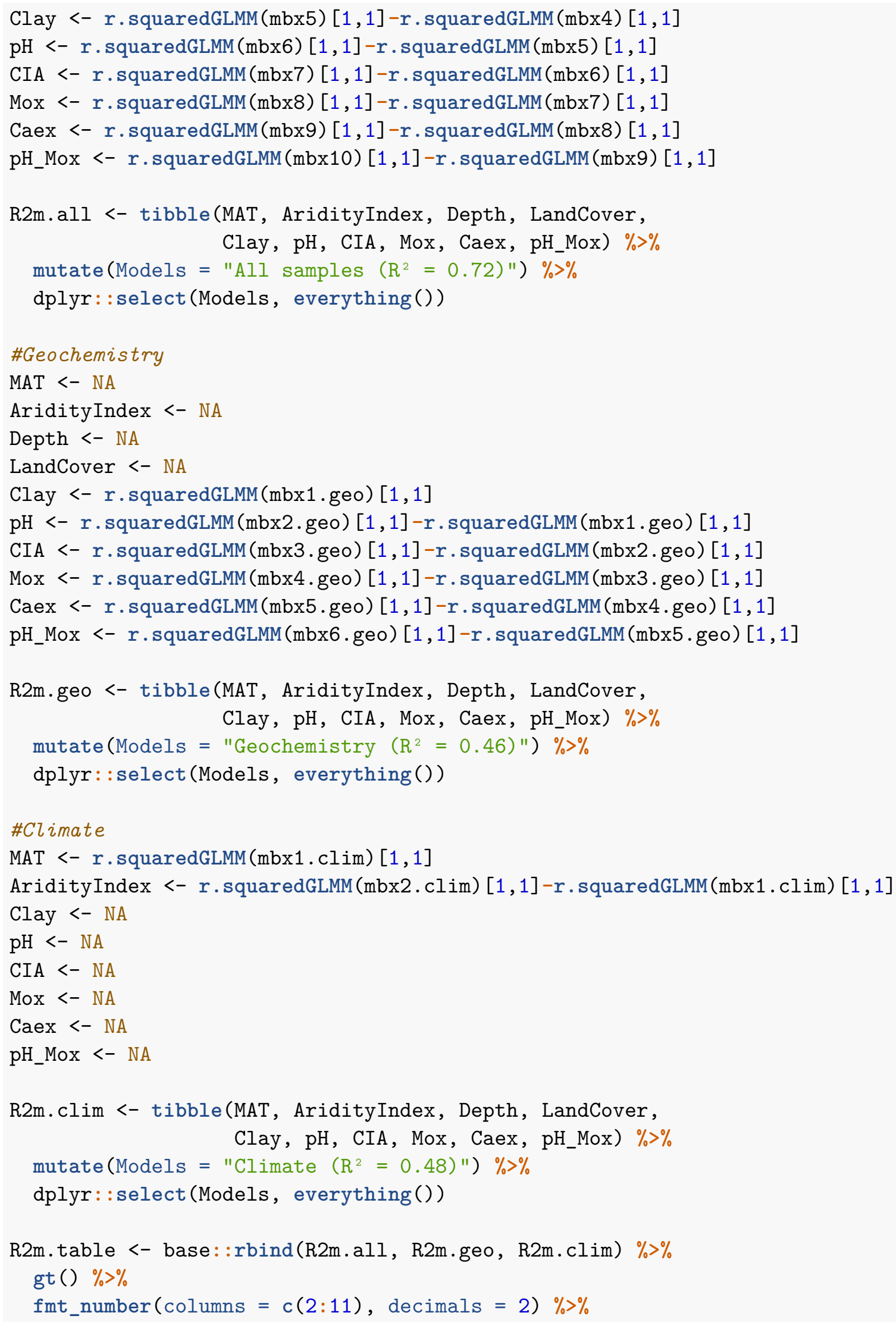




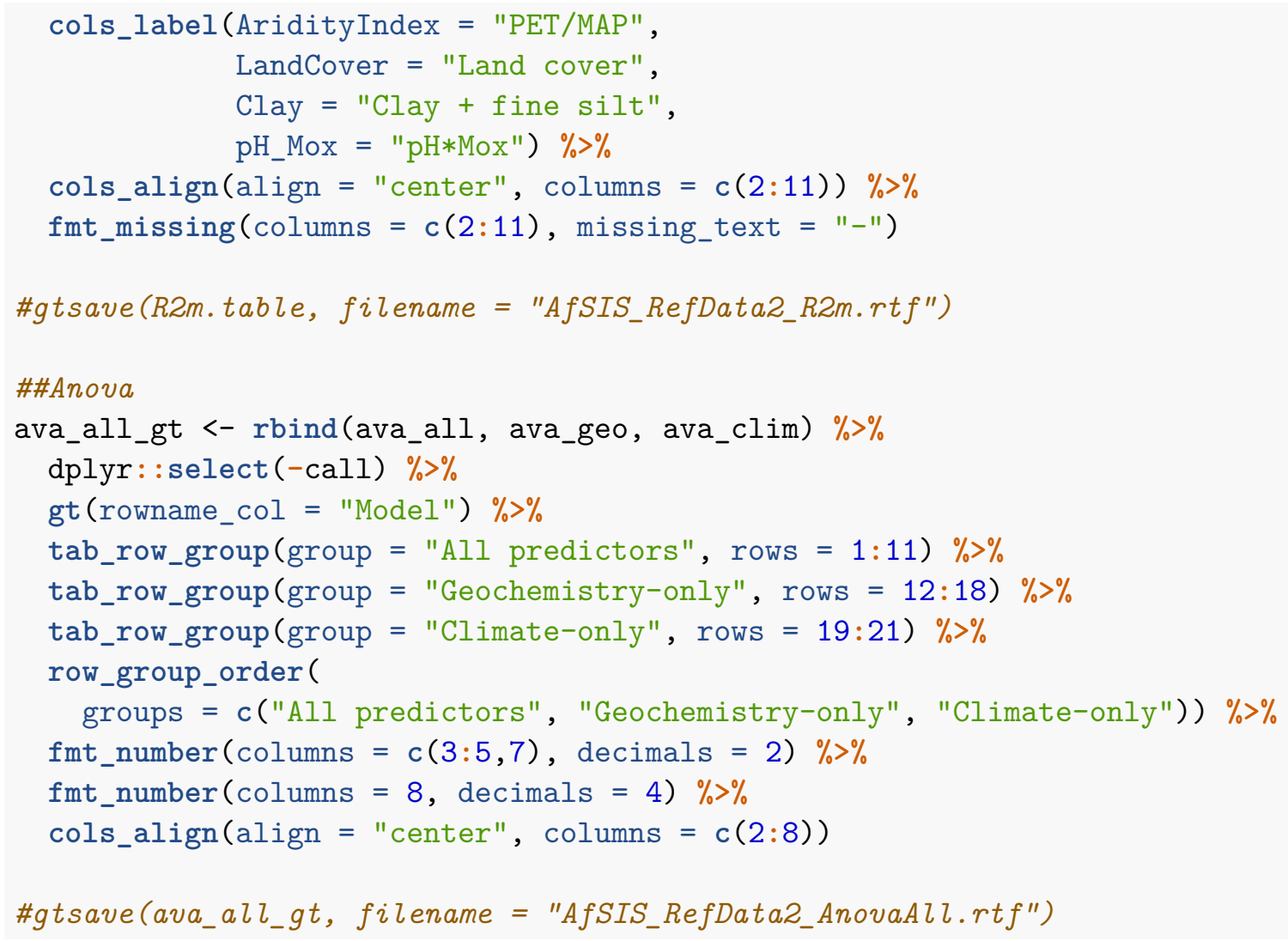

\subsubsection{Variation partitioning}

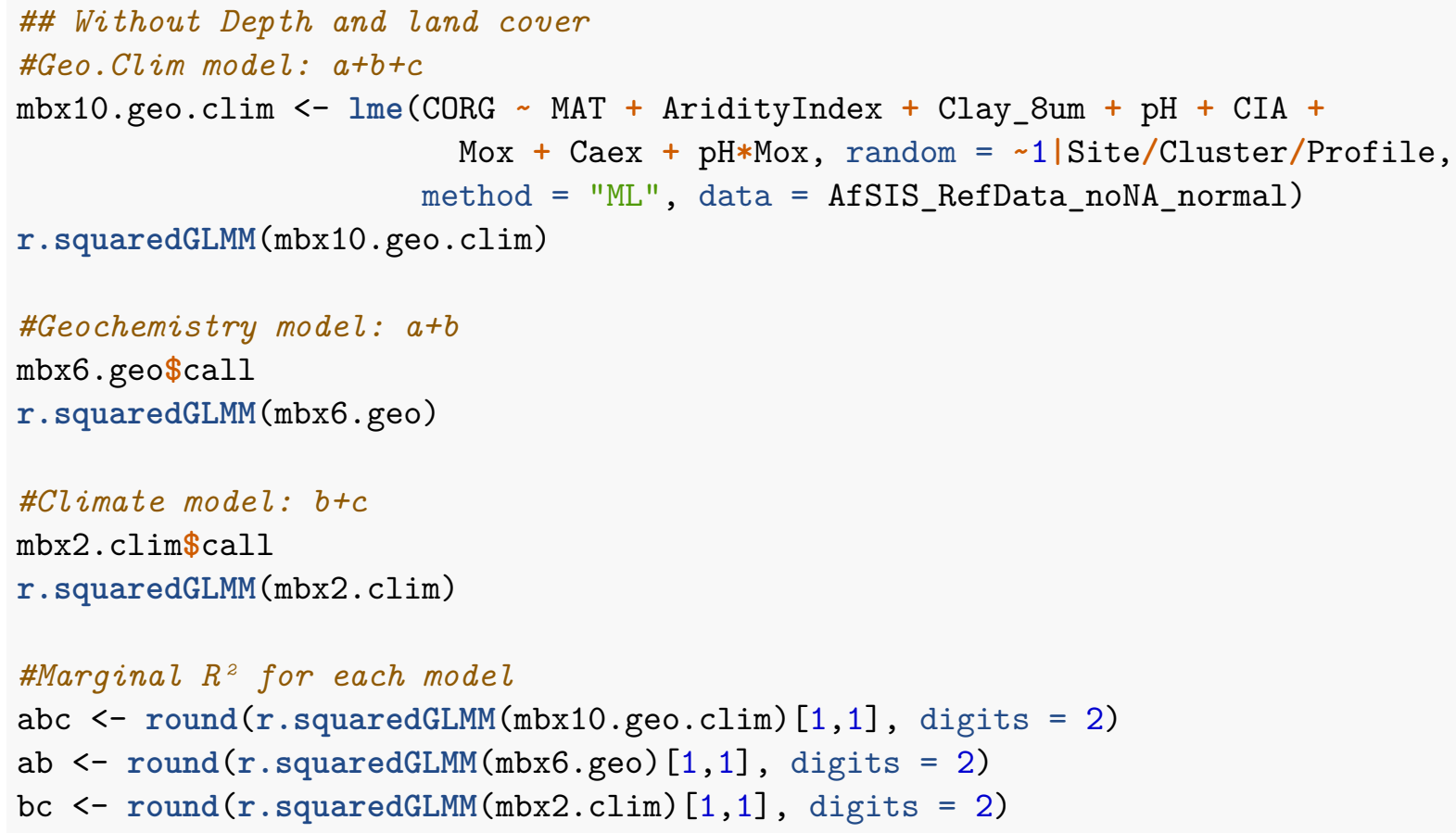




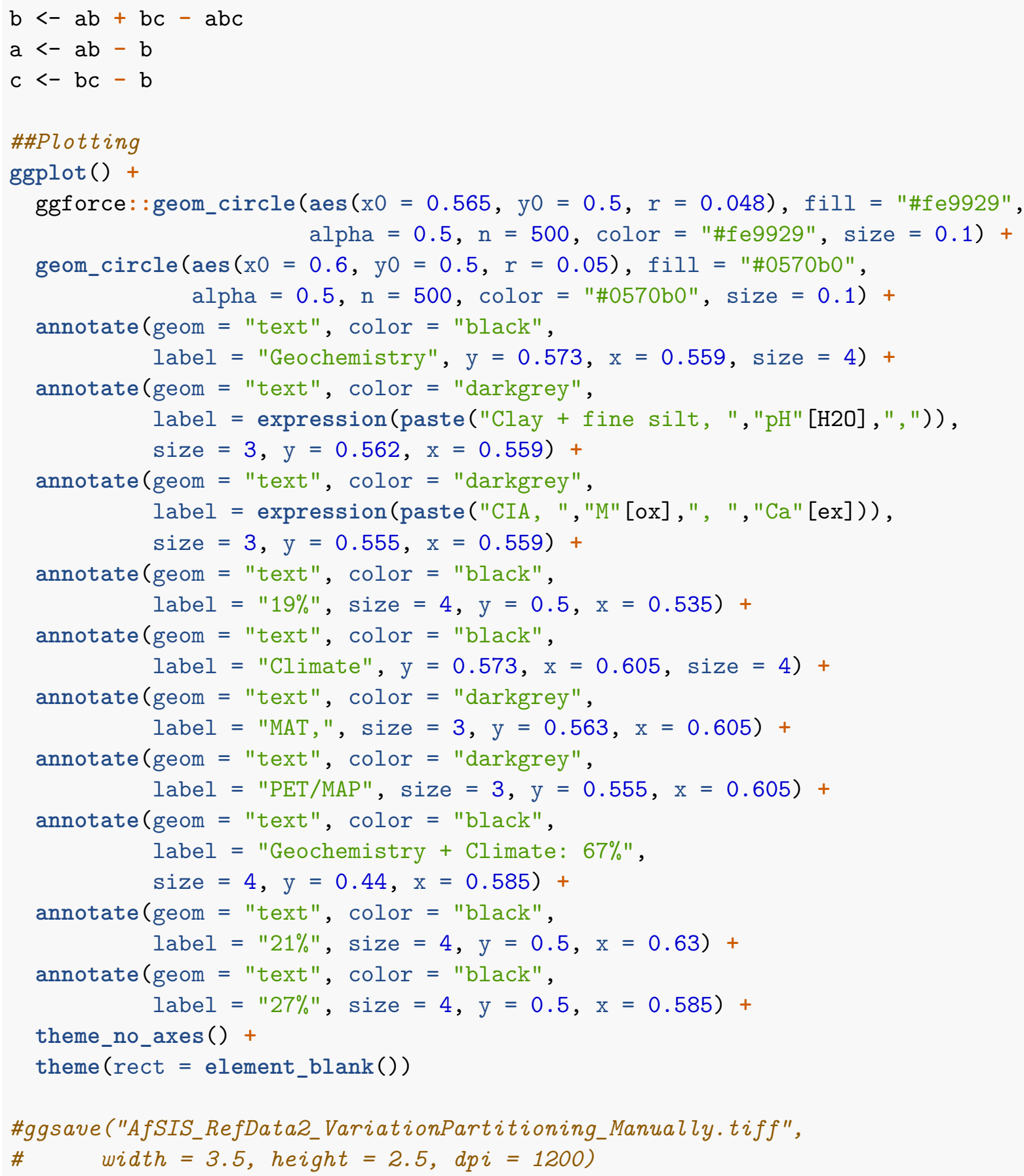

\subsubsection{Clay and land cover models}

\section{Clay-only model (< 8um):}

LMM_Clay <- nlme: :lme(CORG Clay_8um, random = 1|Site/Cluster/Profile, method = "ML", data = AfSIS_RefData_noNA_normal) 
summary (LMM_Clay) \$AIC

\#\# [1] 2979.196

summary(LMM_Clay) $\$$ tTable $\%>\%$ knitr: :kable()

\begin{tabular}{lrrrrr}
\hline & Value & Std.Error & DF & t-value & $p$-value \\
\hline (Intercept) & -0.4036670 & 0.1129380 & 788 & -3.574237 & 0.0003726 \\
Clay_8um & 0.0877243 & 0.0210309 & 349 & 4.171208 & 0.0000383 \\
\hline
\end{tabular}

$\mathrm{R}^{2}$ results of final model:

\#\# $\quad$ R2m $\quad$ R2c

\#\# $[1] \quad 0.008495354 \quad$,

Clay-only model (<20um):

LMM_Clay_20<- nlme::lme(CORG Clay_20um, random $=\sim 1 \mid$ Site/Cluster/Profile, method $=$ "ML", data $=$ AfSIS_RefData_noNA_normal)

summary (LMM_Clay_20) \$AIC

\#\# [1] 2942.657

summary(LMM_Clay_20) $\$$ tTable $\%>\%$ knitr: :kable()

\begin{tabular}{lrrrrr}
\hline & Value & Std.Error & DF & t-value & p-value \\
\hline (Intercept) & -0.3641492 & 0.1049396 & 788 & -3.470082 & 0.0005486 \\
Clay_20um & 0.1647142 & 0.0217156 & 349 & 7.585056 & 0.0000000 \\
\hline
\end{tabular}

$\mathrm{R}^{2}$ results of final model:

\#\# $\quad$ R2m $\quad$ R2c

\#\# [1, ] $0.03233415 \quad 0.6836811$

Land cover-only model:

LMM_LC <- nlme::lme(CORG LandCover, random = $\sim 1 \mid$ Site/Cluster/Profile, method $=$ "ML", data $=$ AfSIS_RefData_noNA_normal)

summary (LMM_LC) \$AIC 
summary (LMM_LC) $\$$ tTable $\%>\%$ knitr: :kable()

\begin{tabular}{lrrrrr}
\hline & Value & Std.Error & DF & t-value & p-value \\
\hline Intercept) & -0.5048850 & 0.1252783 & 785 & -4.0301074 & 0.0000612 \\
LandCoverForest & 0.2855258 & 0.1153232 & 785 & 2.4758744 & 0.0135009 \\
LandCoverGrassland & 0.0428359 & 0.0588019 & 785 & 0.7284775 & 0.4665386 \\
LandCoverOther & 0.0590269 & 0.0499577 & 785 & 1.1815377 & 0.2377470 \\
\hline
\end{tabular}

$\mathrm{R}^{2}$ results of final model:

\#\# $\quad$ R2m $\quad$ R2c

$\begin{array}{llll}\text { \#\# }[1,] & 0.008188464 & 0.7498529\end{array}$

\section{Clay + land cover-only model:}

LMM_Clay_LC <- nlme::lme(CORG Clay_8um + LandCover, random $=\sim 1 \mid$ Site/Cluster/Profile, method $=$ "ML", data $=$ AfSIS_RefData_noNA_normal)

summary (LMM_Clay_LC) \$AIC

\#\# [1] 2977.265

summary (LMM_Clay_LC) $\$$ tTable $\%>\%$ knitr: :kable()

\begin{tabular}{lrrrrr}
\hline & Value & Std.Error & DF & t-value & p-value \\
\hline (Intercept) & -0.4734388 & 0.1177611 & 785 & -4.0203314 & 0.0000637 \\
Clay_8um & 0.0911308 & 0.0210673 & 349 & 4.3256952 & 0.0000199 \\
LandCoverForest & 0.2941654 & 0.1133265 & 785 & 2.5957335 & 0.0096153 \\
LandCoverGrassland & 0.0529993 & 0.0579815 & 785 & 0.9140715 & 0.3609600 \\
LandCoverOther & 0.0758906 & 0.0494496 & 785 & 1.5347039 & 0.1252595 \\
\hline
\end{tabular}

$\mathrm{R}^{2}$ results of final model:

$\begin{array}{rrr}\# \# & R 2 m & R 2 c \\ \# \# \quad[1,] & 0.02154288 & 0.713932\end{array}$

\subsection{Sub-models}

This section contains the code for all sub-models. The results can be found in Figure 4 and Table B2. 


\subsubsection{Depth models}

This section contains the two models for the topsoil and subsoil samples, respectively.

\section{Normalize and standardize each sub-group:}

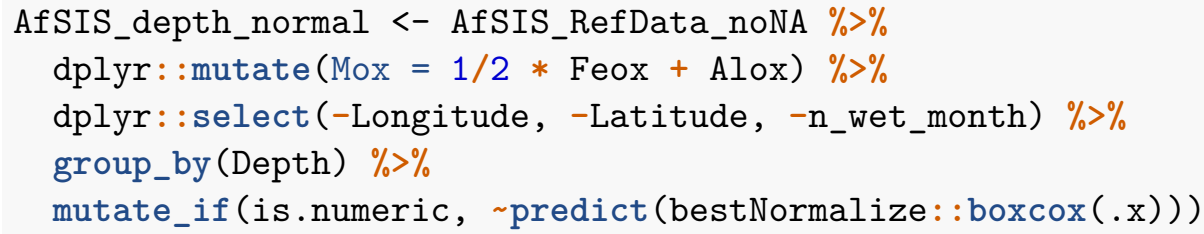

\section{Topsoil:}

Build models:

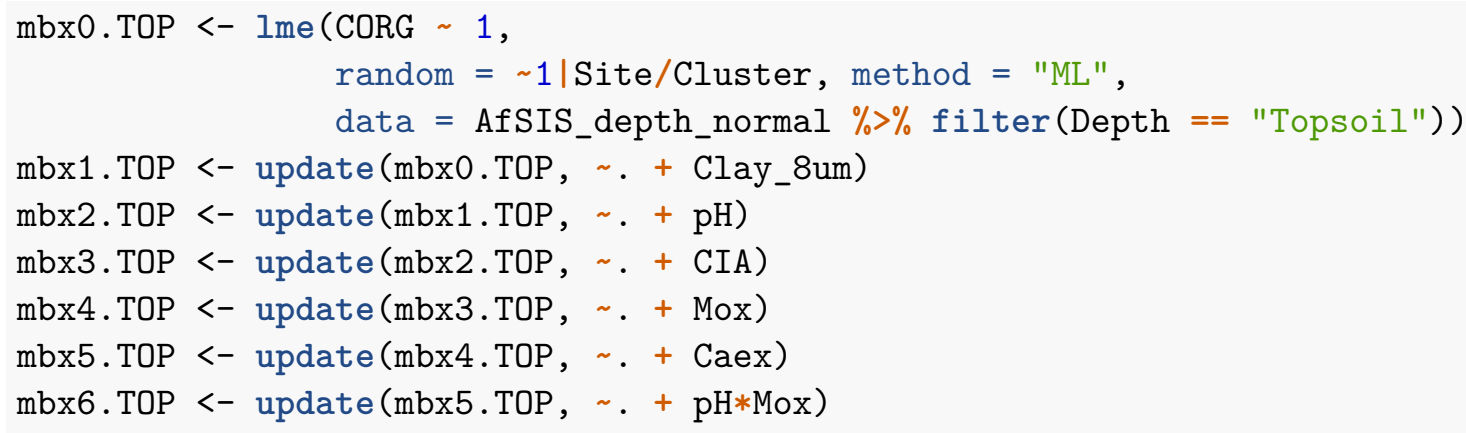

Autocorrelation:

vif_top <- as.data.frame (car: :vif (mbx6.TOP))

vif_top_test <- $\max \left(v i f \_t o p \$ " c a r:: v i f(\operatorname{mbx} 6 . T 0 P) "\right)<3.0$

VIF < 3.0: TRUE -> no autocorrelation

Diagnostic plots:

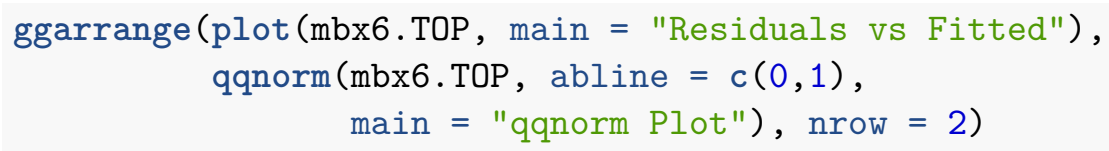




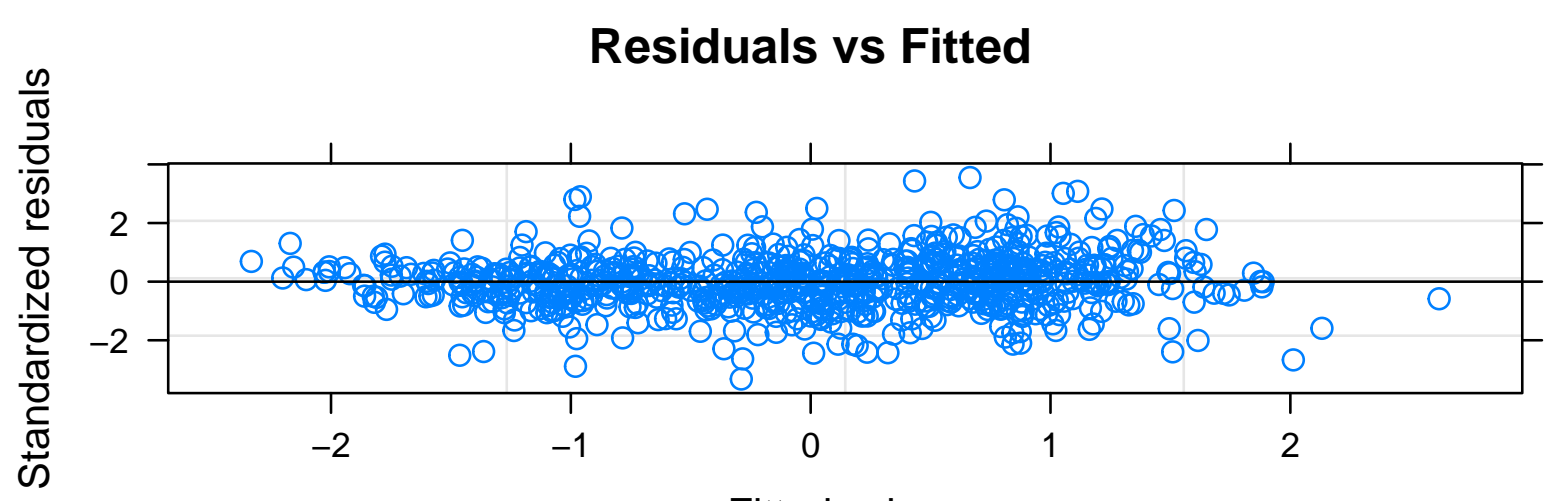

Fitted values

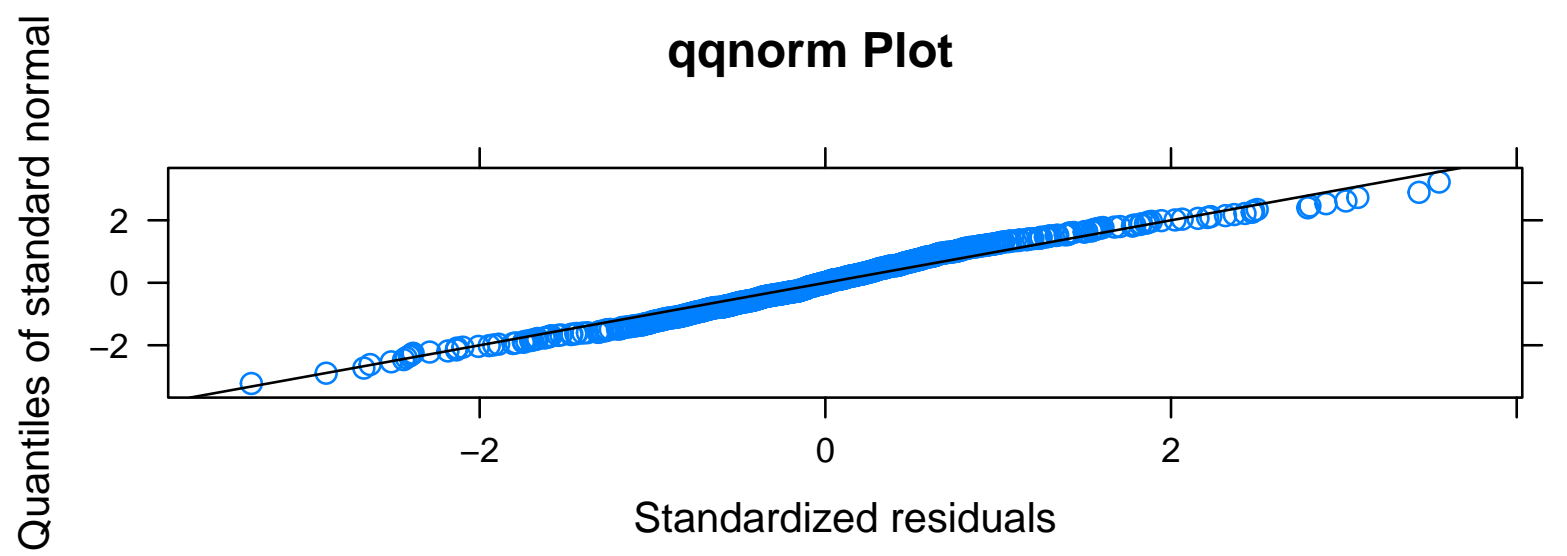

Model assumptions are met.

Anova output for all topsoil models (step-wise):

summary (mbx6.TOP)

\#\# Linear mixed-effects model fit by maximum likelihood

\#\# Data: AfSIS_depth_normal \% $\%$ filter(Depth == "Topsoil")

\#\# AIC BIC logLik

$\begin{array}{llll}\text { \#\# } \quad 967.1072 & 1013.84 & -473.5536\end{array}$

\#\#

\#\# Random effects:

\#\# Formula: $\sim 1$ | Site

\#\# (Intercept)

\#\# StdDev: 0.4680077

\#\#

\#\# Formula: $\sim 1$ | Cluster \%in\% Site

\#\# (Intercept) Residual

$\begin{array}{lll}\text { \#\# StdDev: } 0.2141417 & 0.3675949\end{array}$

\#\#

\#\# Fixed effects: CORG Clay_8um + pH + CIA + Mox + Caex + pH:Mox

\#\# Value Std.Error DF t-value p-value

$\begin{array}{lllllll}\text { \#\# (Intercept) } & -0.2490844 & 0.07838700 & 425 & -3.177624 & 0.0016\end{array}$ 


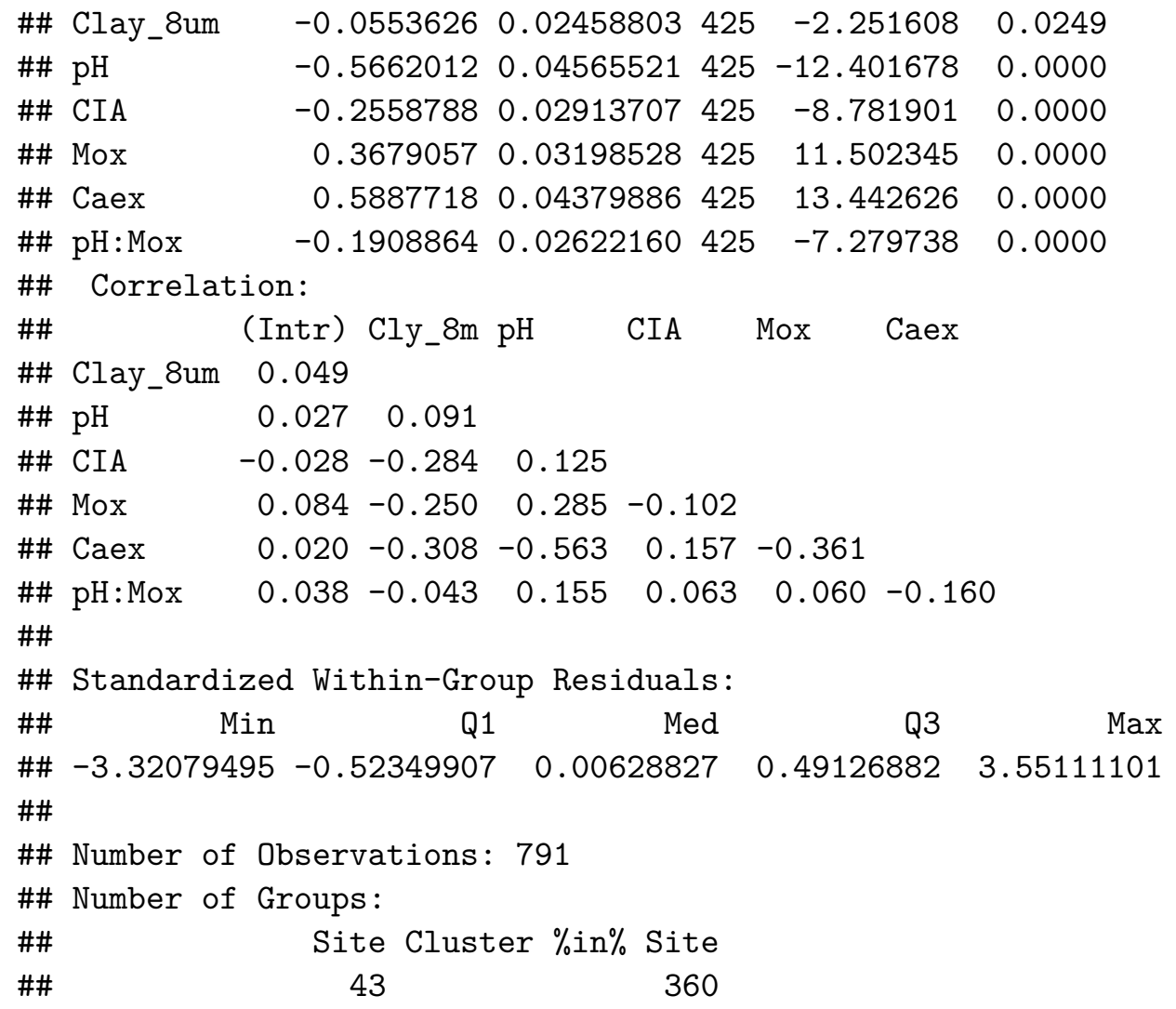

\section{Subsoil:}

Build model: 


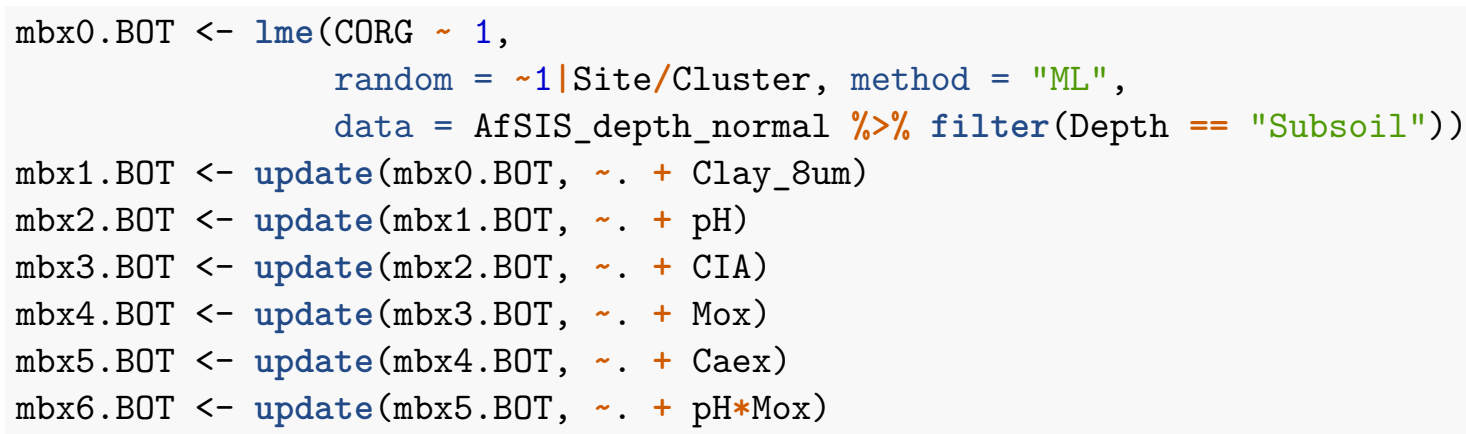

Autocorrelation:

vif_bot <- as.data.frame(vif (mbx6.BOT))

vif_bot_test <- $\max ($ vif_bot $\$ " v i f(\operatorname{mbx} 6 . B 0 T) ")<3.0$

VIF < 3.0: TRUE -> no autocorrelation

Diagnostic plots:
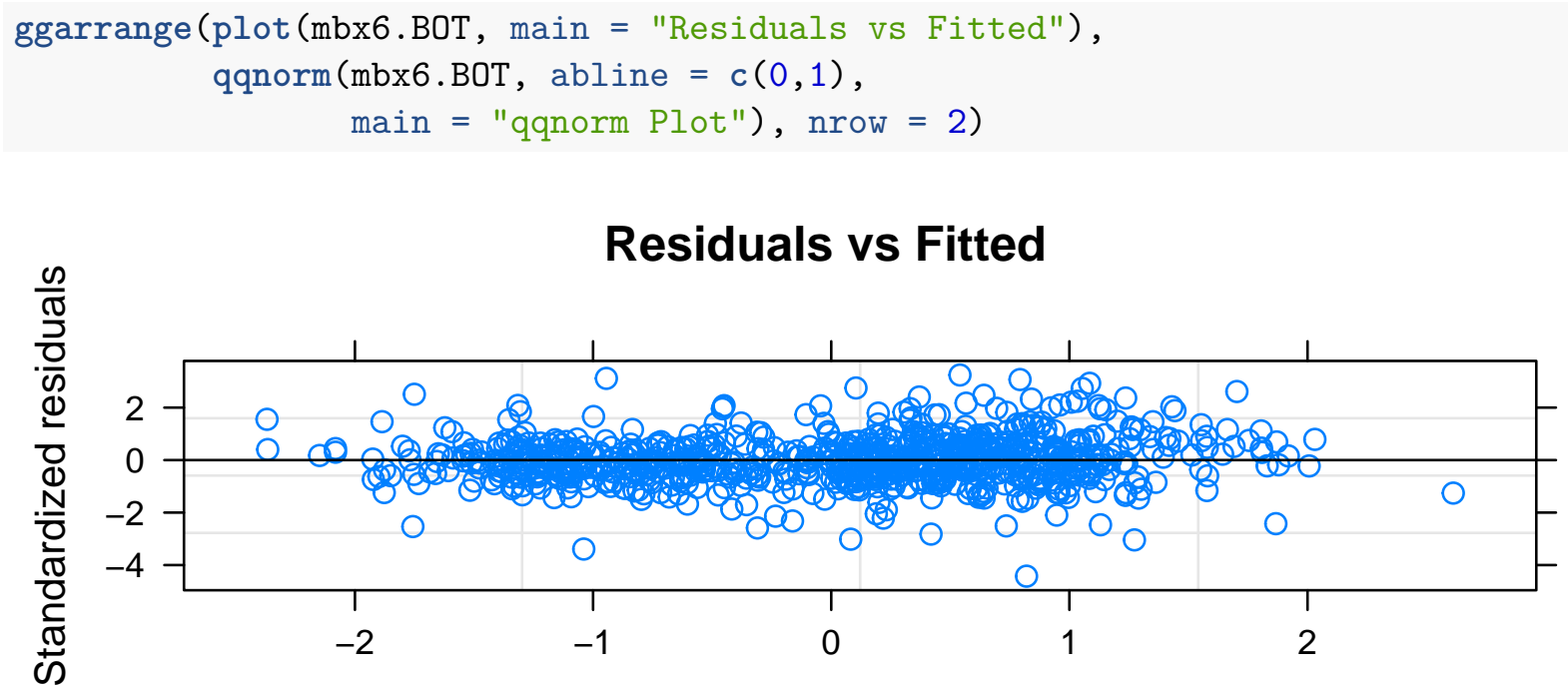

Fitted values

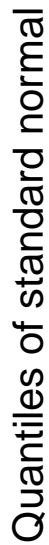

qqnorm Plot

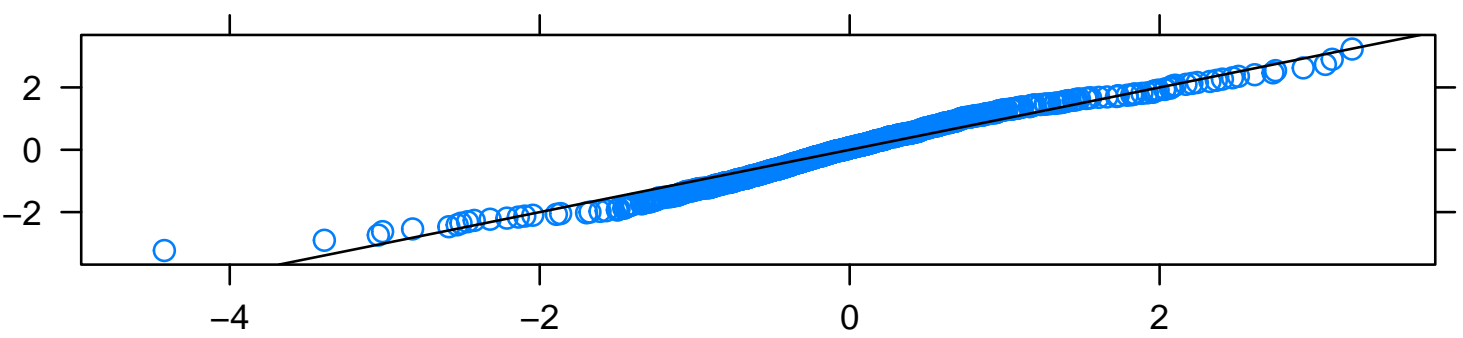

Standardized residuals 
Model assumptions are met.

Anova output for all subsoil models (step-wise):

summary (mbx6. BOT)

\#\# Linear mixed-effects model fit by maximum likelihood

\#\# Data: AfSIS_depth_normal \% $\%$ filter(Depth == "Subsoil")

\#\# AIC BIC logLik

\#\# $\quad 1106.115 \quad 1153.085 \quad-543.0574$

\#\#

\#\# Random effects:

\#\# Formula: 1 | Site

\#\# (Intercept)

\#\# StdDev: 0.4897167

\#\#

\#\# Formula: 1 | Cluster \%in\% Site

\#\# (Intercept) Residual

\#\# StdDev: 0.25429650 .3891222

\#\#

\#\# Fixed effects: CORG Clay_8um + pH + CIA + Mox + Caex + pH:Mox

\#\# Value Std.Error DF t-value $p$-value

\#\# (Intercept) $-0.1983574 \quad 0.08284013 \quad 446 \quad-2.394461 \quad 0.0171$

$\begin{array}{lllllll}\text { \#\# Clay_8um } & 0.0455868 & 0.02635944 & 446 & 1.729428 & 0.0844\end{array}$

$\begin{array}{llllll}\# \# \mathrm{pH} & -0.3290670 & 0.04712241 & 446 & -6.983238 & 0.0000\end{array}$

$\begin{array}{llllll}\text { \#\# CIA } & -0.1040680 & 0.03215945 & 446 & -3.236000 & 0.0013\end{array}$

$\begin{array}{lllllll}\text { \#\# Mox } & 0.4147492 & 0.03339600 & 446 & 12.419131 & 0.0000\end{array}$

$\begin{array}{lllllll}\text { \#\# Caex } & 0.4186212 & 0.04937509 & 446 & 8.478390 & 0.0000\end{array}$

$\begin{array}{lllllll}\text { \#\# } \mathrm{pH}: \operatorname{Mox} & -0.1517777 & 0.02667293 & 446 & -5.690326 & 0.0000\end{array}$

\#\# Correlation:

\#\# (Intr) $\mathrm{Cly}_{-} 8 \mathrm{~m} \mathrm{pH} \quad$ CIA Mox Caex

\#\# Clay_8um 0.055

$\begin{array}{lll}\# \# \mathrm{pH} & -0.010 & 0.054\end{array}$

$\begin{array}{llll}\text { \#\# CIA } & -0.041 & -0.222 & 0.205\end{array}$

$\begin{array}{llllll}\text { \#\# Mox } & 0.075 & -0.283 & 0.177 & -0.202\end{array}$

$\begin{array}{llllll}\text { \#\# Caex } & 0.029 & -0.303 & -0.558 & 0.101 & -0.298\end{array}$

$\begin{array}{llllllll}\text { \#\# } \mathrm{pH}: \operatorname{Mox} & 0.041 & 0.005 & 0.261 & 0.034 & -0.027 & -0.166\end{array}$

\#\#

\#\# Standardized Within-Group Residuals:

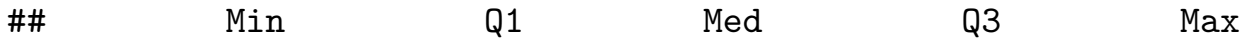

$\begin{array}{llllll}\# \# & -4.42191470 & -0.51362238 & -0.04447791 & 0.48136537 & 3.24220899\end{array}$

\#\#

\#\# Number of Observations: 810

\#\# Number of Groups:

\#\#

\#\#

Site Cluster \%in\% Site

$42 \quad 358$ 
ava_BOT <- anova (mbx0.BOT, mbx1.BOT, mbx2.BOT, mbx3.BOT, mbx4.BOT, mbx5 .BOT, mbx6.BOT) ava_BOT

\begin{tabular}{|c|c|c|c|c|c|c|c|c|c|}
\hline \#\# & Model & $d f$ & AIC & BIC & $\operatorname{logLik}$ & Tes & & L.Ratio & $\mathrm{p}$-value \\
\hline \#\# mbx0.BOT & 1 & 4 & 1460.723 & 1479.512 & -726.3617 & & & & \\
\hline \#\# mbx1.BОT & 2 & 5 & 1373.421 & 1396.907 & -681.7107 & $1 \mathrm{vs}$ & 2 & 89.30198 & $<.0001$ \\
\hline \#\# mbx2.BOT & 3 & 6 & 1372.978 & 1401.160 & -680.4888 & $2 \mathrm{vs}$ & 3 & 2.44387 & 0.1180 \\
\hline \#\# mbx3.BOT & 4 & 7 & 1373.422 & 1406.301 & -679.7109 & $3 \mathrm{vs}$ & 4 & 1.55580 & 0.2123 \\
\hline \#\# mbx4.BOT & 5 & 8 & 1188.602 & 1226.178 & -586.3011 & $4 \mathrm{vs}$ & 5 & 186.81959 & $<.0001$ \\
\hline \#\# mbx5.B0T & 6 & 9 & 1135.713 & 1177.987 & -558.8567 & $5 \mathrm{vs}$ & 6 & 54.88874 & $<.0001$ \\
\hline \#\# mbx6.BOT & 7 & 10 & 1106.115 & 1153.085 & -543.0574 & $6 \mathrm{vs}$ & 7 & 31.59862 & $<.0001$ \\
\hline \multicolumn{10}{|c|}{ r.squaredGLMM (mbx6.BOT) } \\
\hline \#\# & R2m & & R2c & & & & & & \\
\hline$\# \#[1] \quad 0.43$, & 320004 & & 3549 & & & & & & \\
\hline
\end{tabular}

\section{Anova Summary table:}

Table B5 in the supplement of the manuscript.

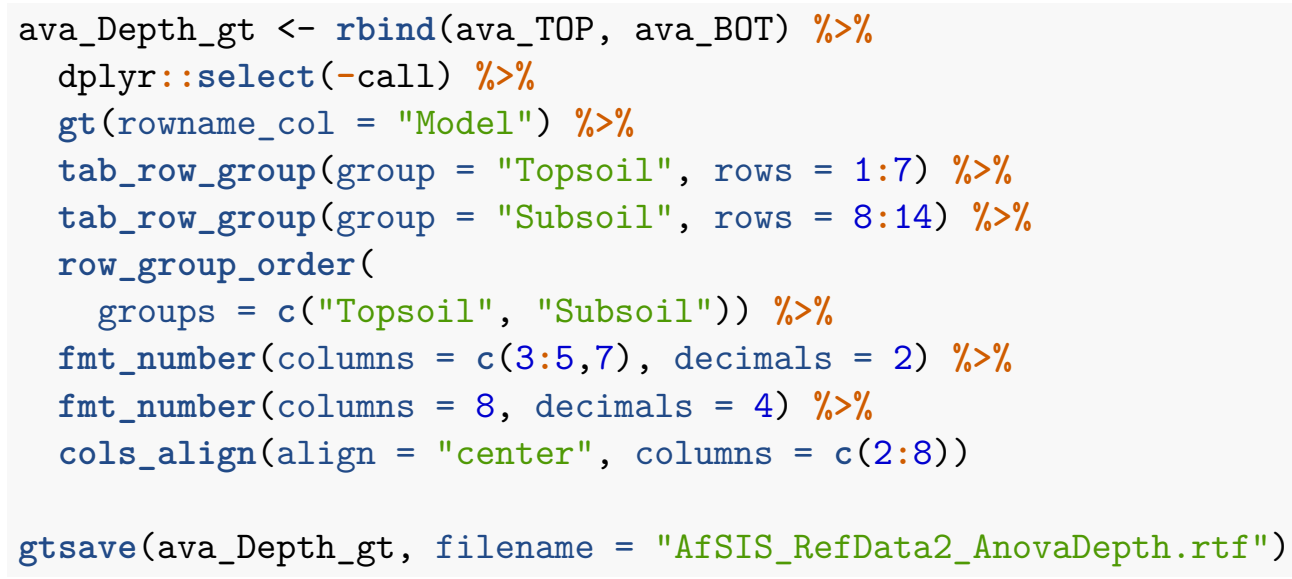

\subsection{2 $\mathrm{pH}_{\mathrm{H} 2 \mathrm{O}}$ models}

This section contains the code for the four $\mathrm{pH}$ models.

\section{Normalize and standardize each sub-group:}

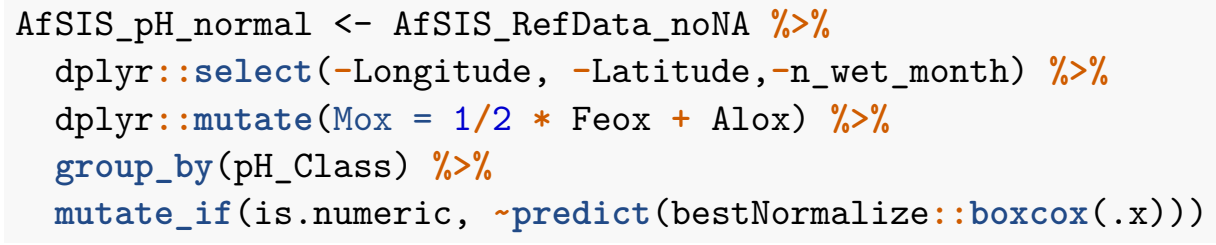


\#\# 'mutate_if()' ignored the following grouping variables:

\#\# Column 'pH_Class'

\section{Strongly acidic:}

\section{Build models:}

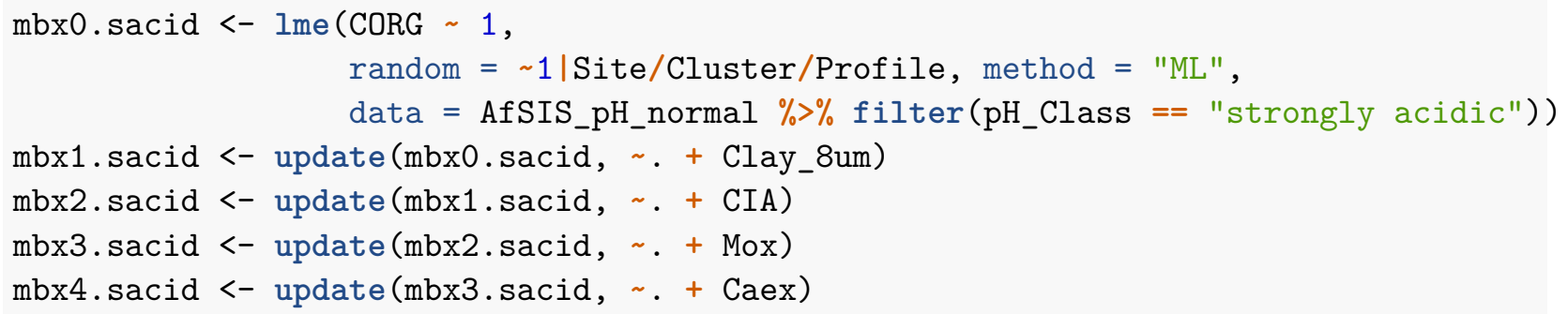

Autocorrelation:

vif_sacid <- as.data.frame(vif (mbx4.sacid))

vif_sacid_test <- $\max ($ vif_sacid $\$ " v i f(\operatorname{mbx} 4$. sacid)" $)<3.0$

VIF < 3.0: TRUE -> no autocorrelation

Diagnostic plots:

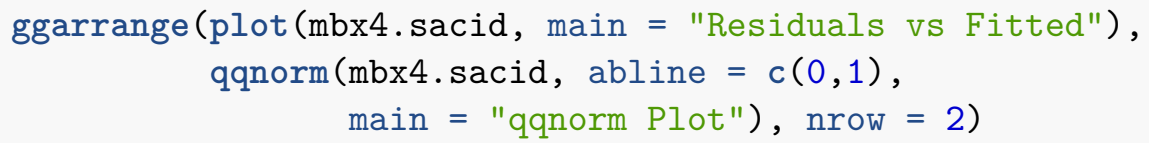




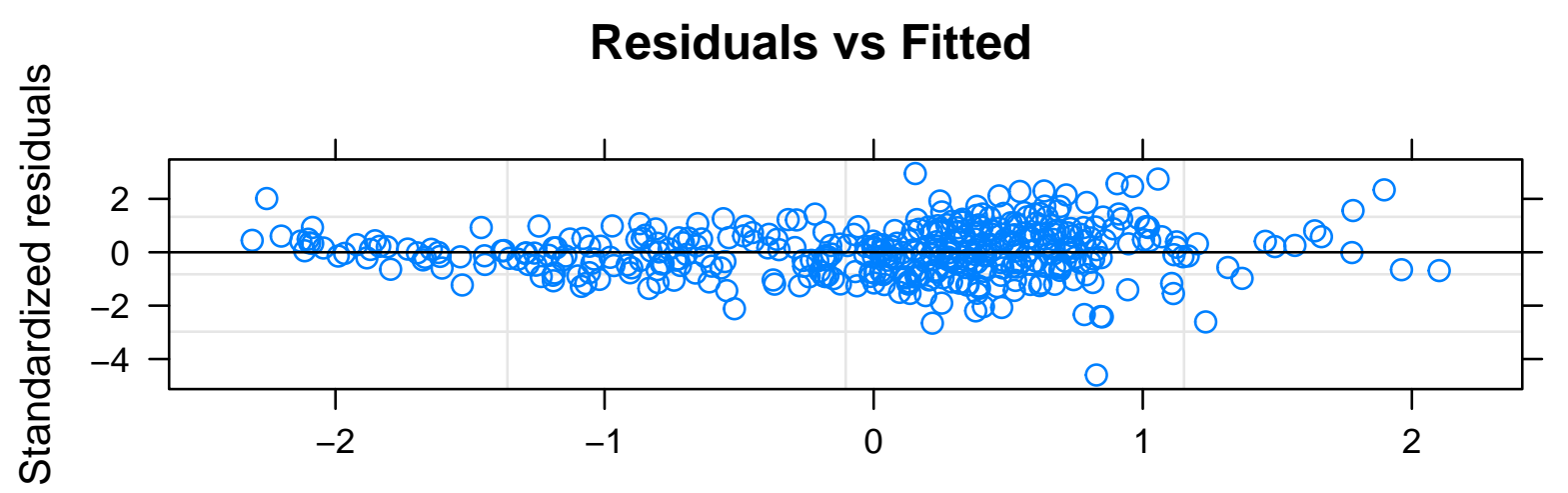

Fitted values

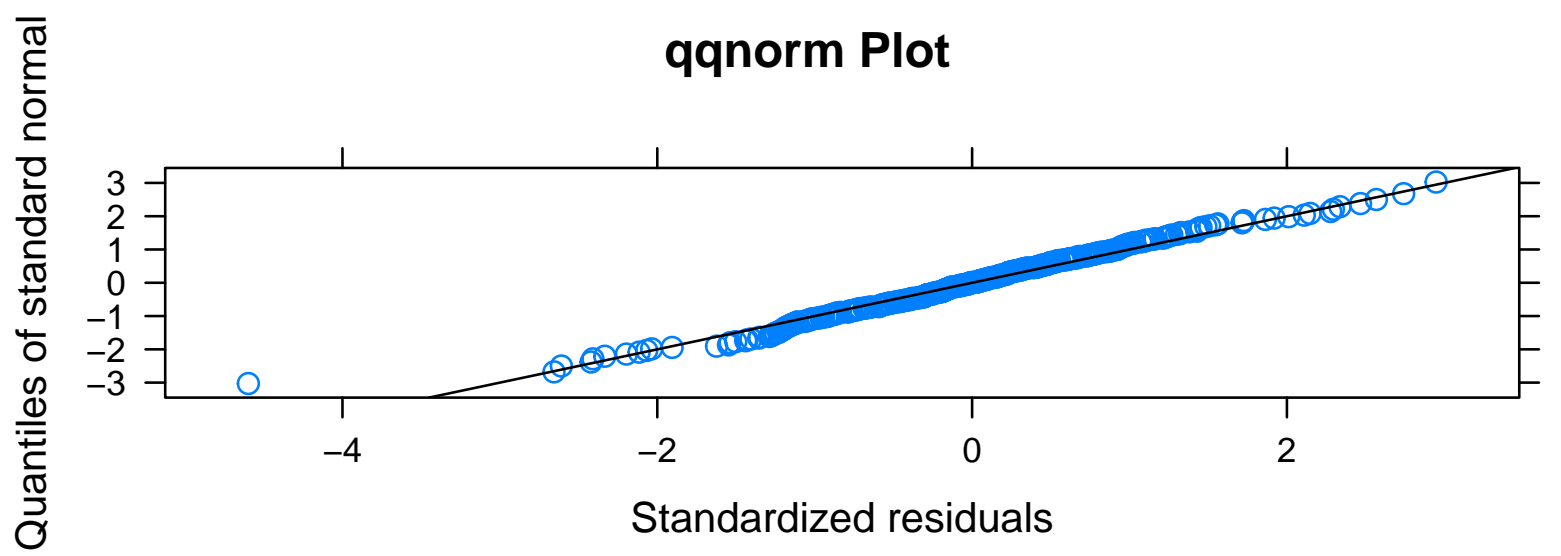

Model assumptions are met. The first plot is a little bit borderline.

Anova output for all strongly acid models (step-wise):

summary (mbx4.sacid)

\#\# Linear mixed-effects model fit by maximum likelihood

\#\# Data: AfSIS_pH_normal $\% \%$ filter(pH_Class == "strongly acidic")

\#\# AIC BIC logLik

\#\# $\quad 690.6762726 .689-336.3381$

\#\#

\#\# Random effects:

\#\# Formula: 1 | Site

\#\# (Intercept)

\#\# StdDev: 0.4719538

\#\#

\#\# Formula: $\sim 1$ | Cluster \%in\% Site

\#\# (Intercept)

\#\# StdDev: 0.2024134

\#\#

\#\# Formula: 1 | Profile \%in\% Cluster \%in\% Site

\#\# (Intercept) Residual

\#\# StdDev: 7.007453e-05 0.4991287 


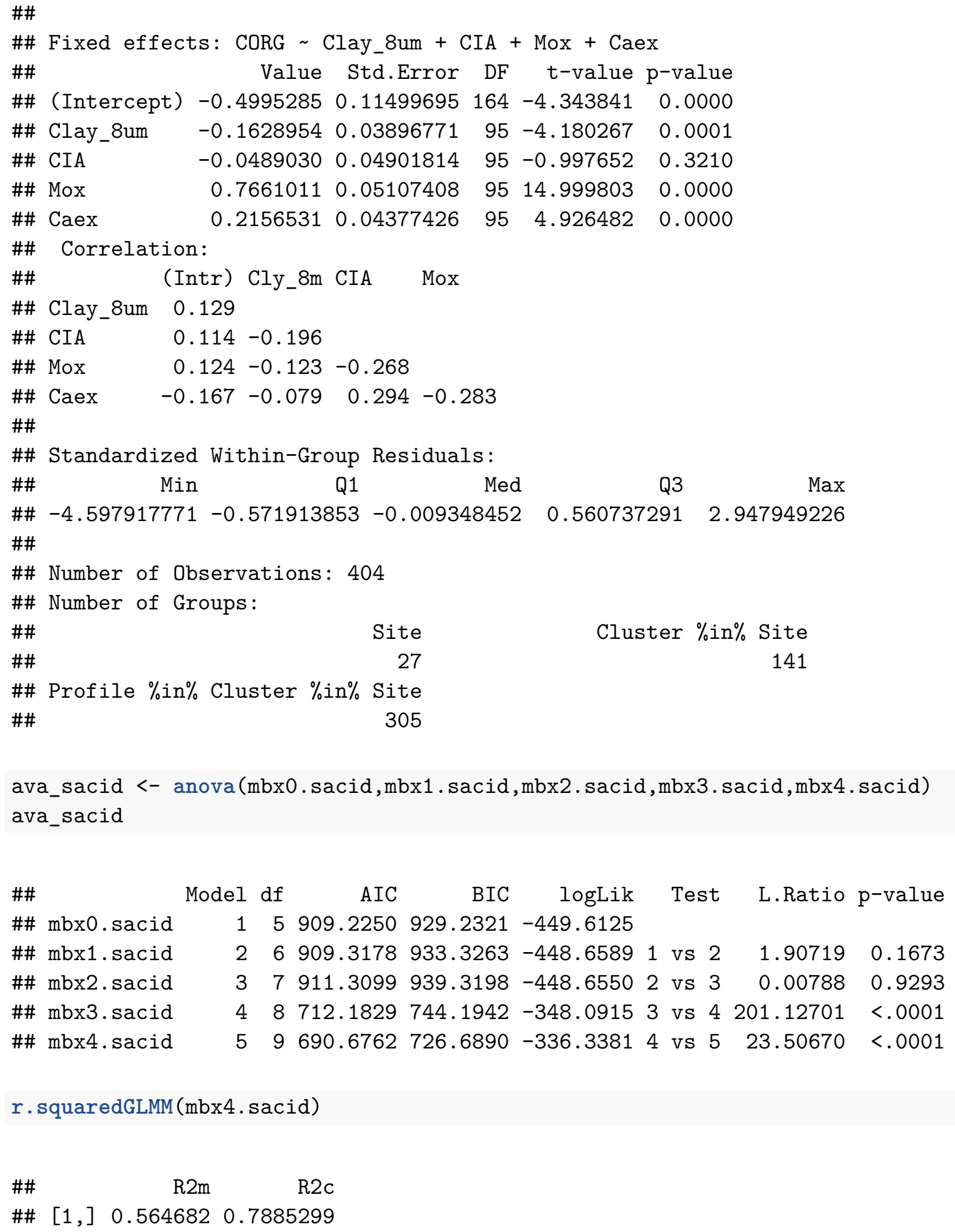

Moderately acidic:

Build models: 


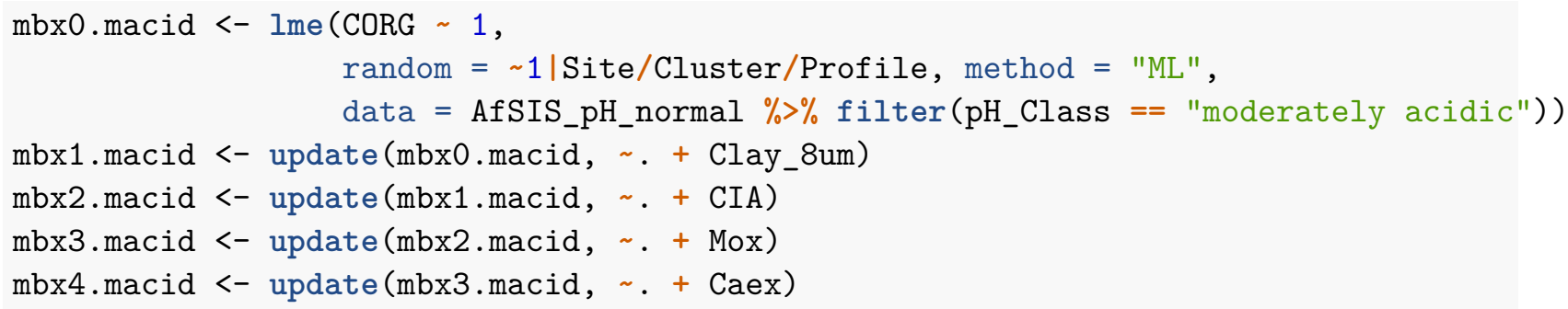

Autocorrelation:

vif_macid <- as.data.frame (vif (mbx4.macid))

vif_macid_test <- $\max \left(v i f \_m a c i d \$ " v i f(\operatorname{mbx} 4 . \operatorname{macid}) "\right)<3.0$

VIF < 3.0: TRUE -> no autocorrelation

Diagnostic plots:
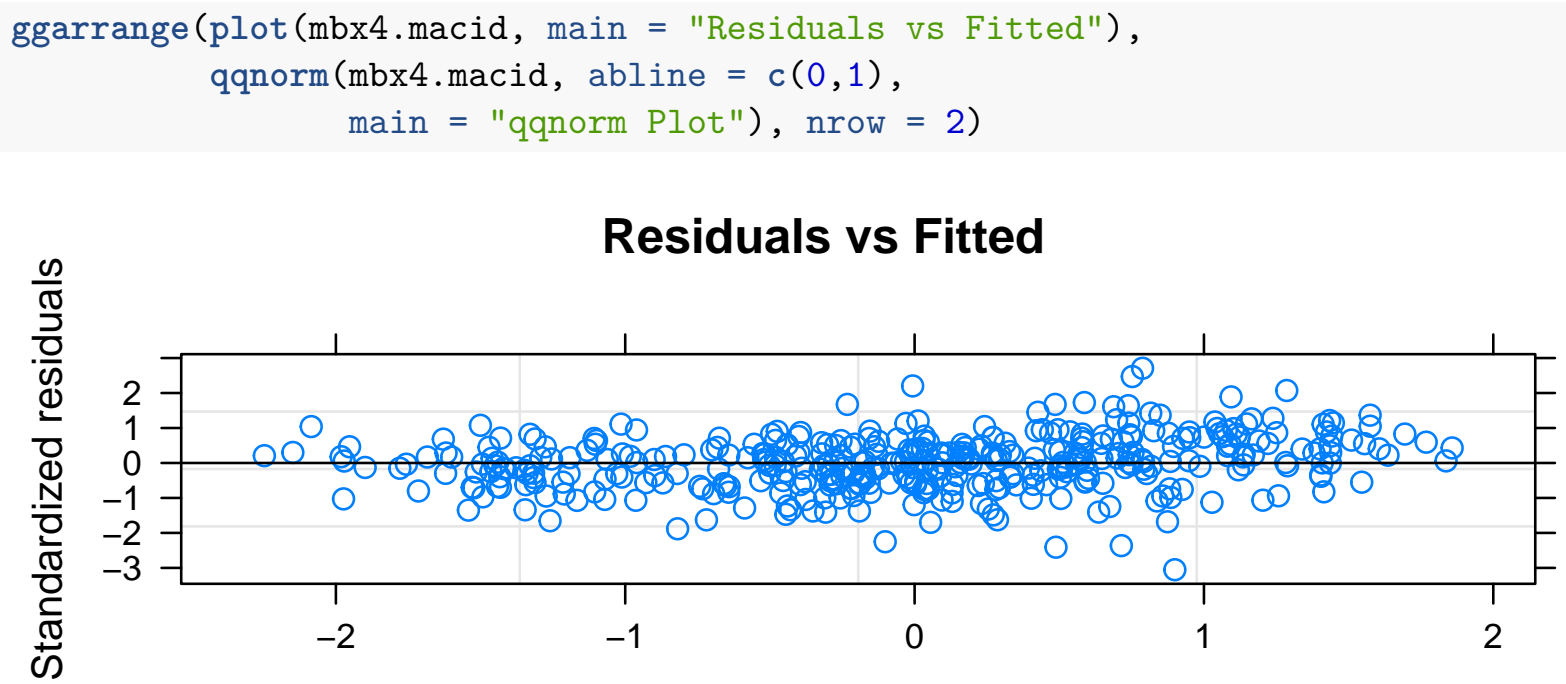

Fitted values

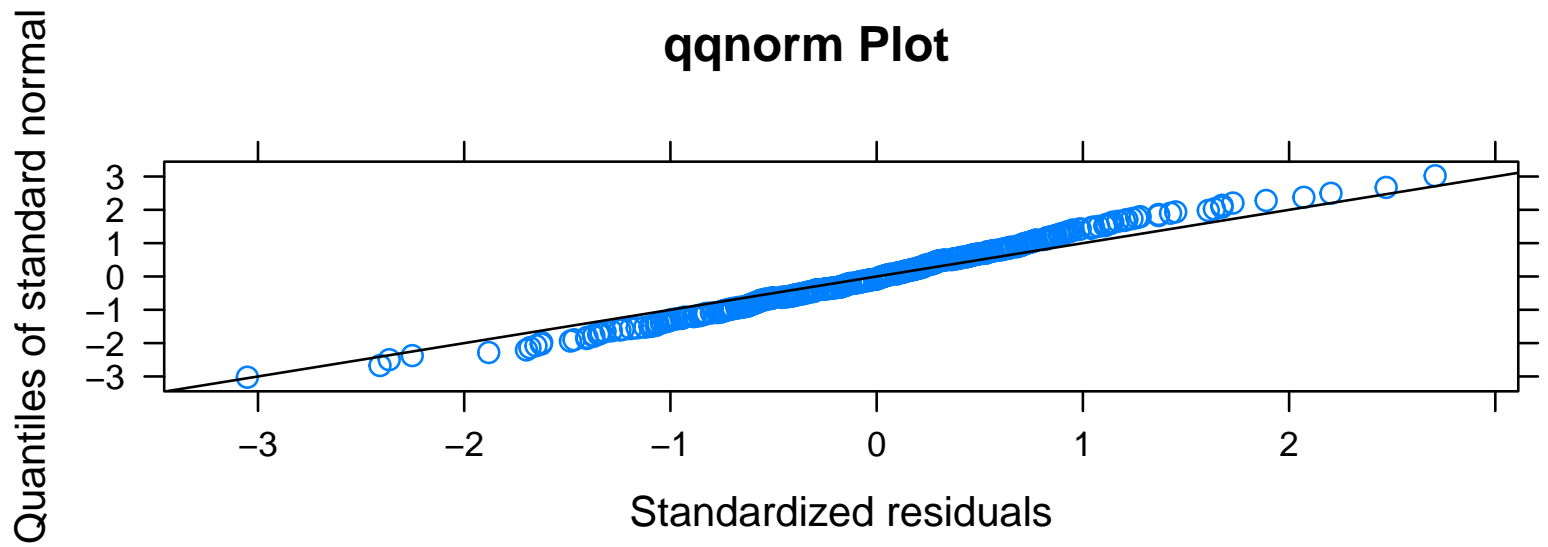

Model assumptions are met.

Anova output for all moderately acid models (step-wise): 


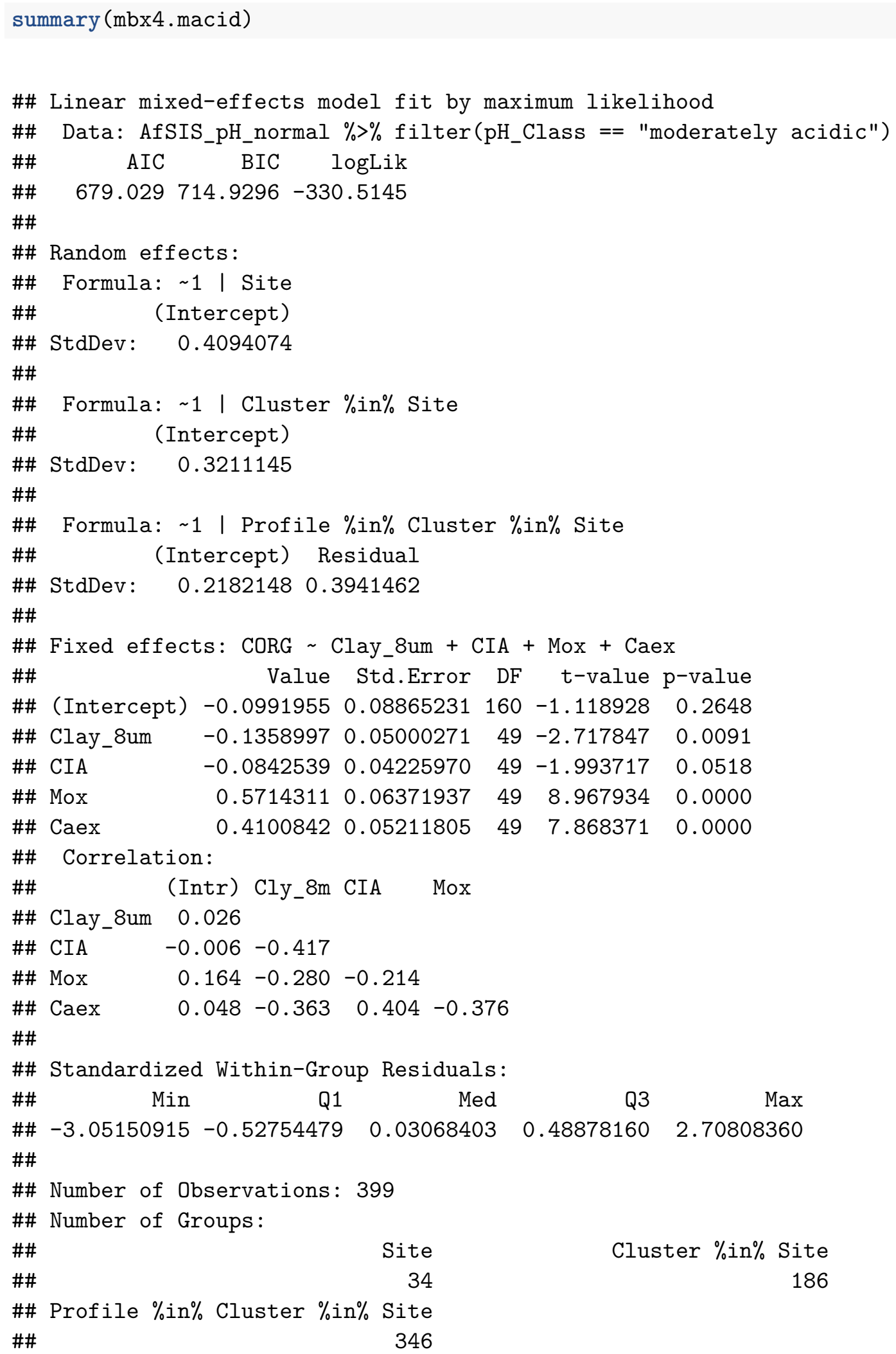




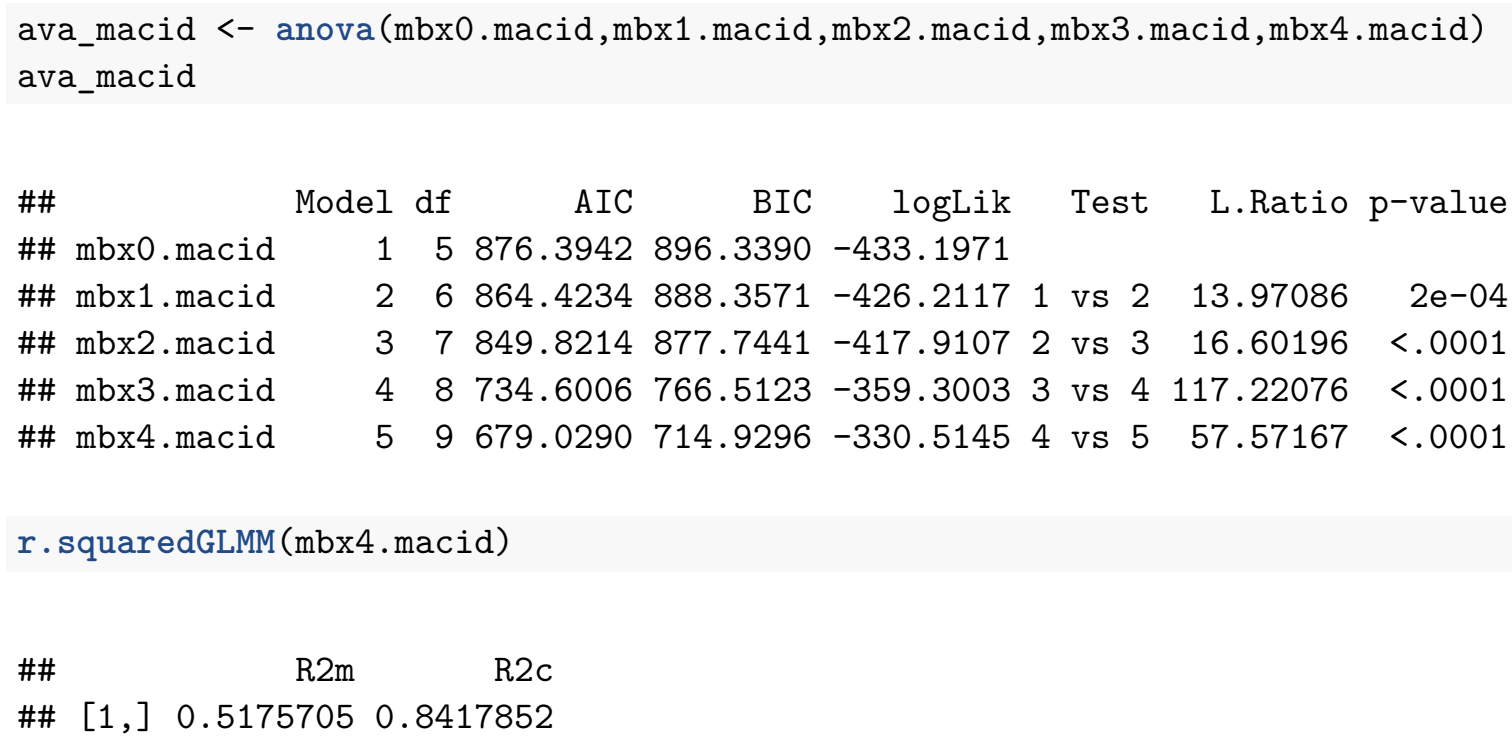

\section{Neutral:}

Build models:

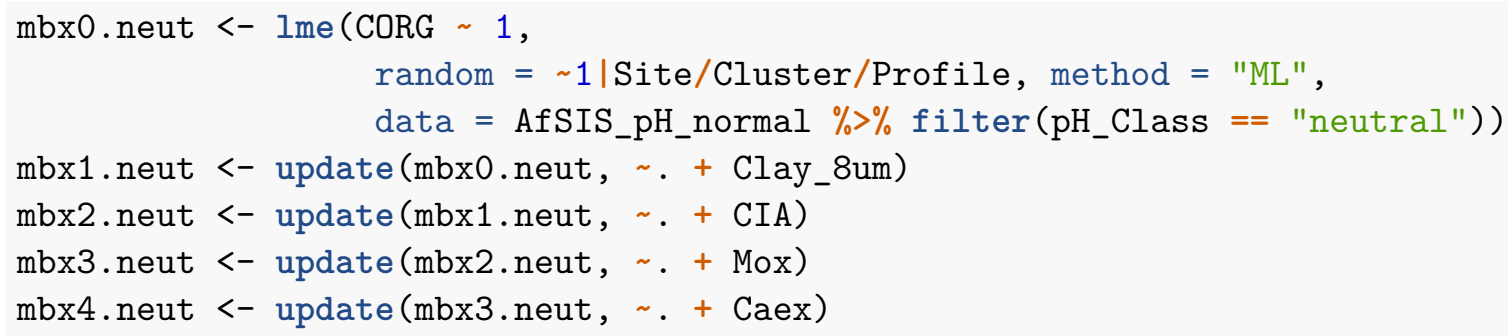

\section{Autocorrelation:}

vif_neut <- as.data.frame (vif (mbx4.neut))

vif_neut_test <- $\max \left(v i f \_n e u t \$ " v i f(\operatorname{mbx} 4 . n e u t) "\right)<3.0$

VIF < 3.0: TRUE -> no autocorrelation

Diagnostic plots:

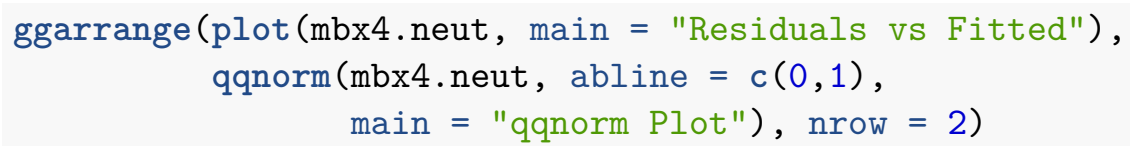




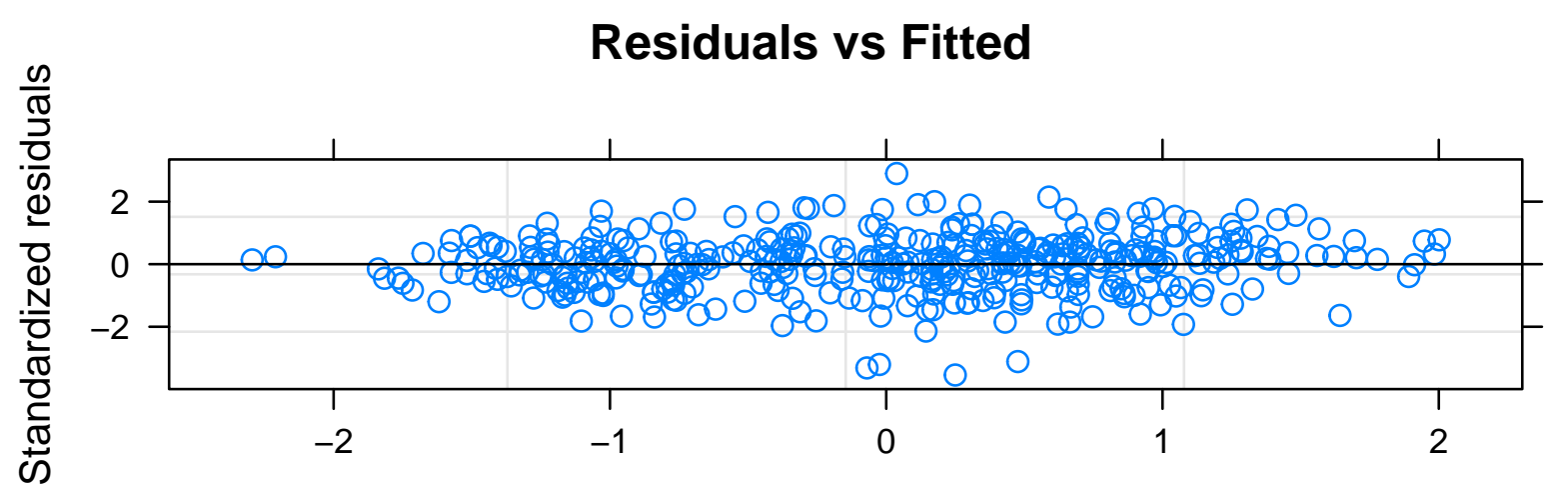

Fitted values

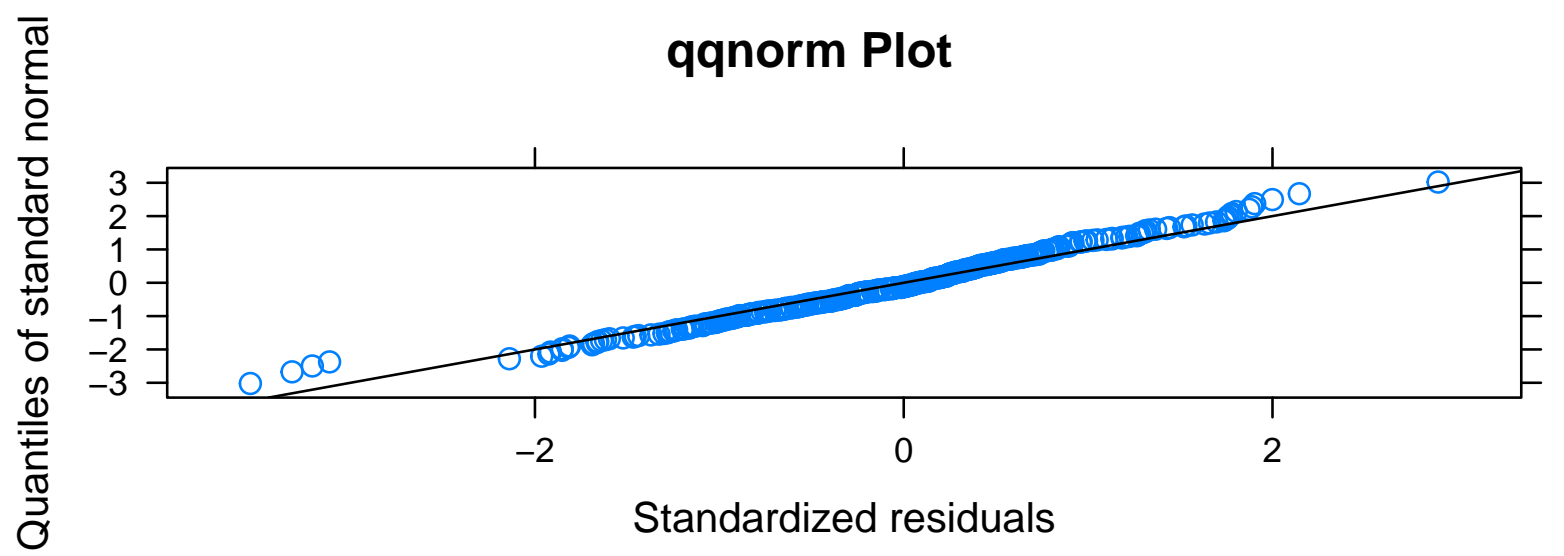

Model assumptions are met.

Anova output for all neutral models (step-wise):

summary (mbx4.neut)

\#\# Linear mixed-effects model fit by maximum likelihood

\#\# Data: AfSIS_pH_normal \% $\% \%$ filter ( $\mathrm{pH}$ _Class == "neutral")

\#\# AIC BIC logLik

\#\# $\quad 581.0317 \quad 616.9097 \quad-281.5158$

\#\#

\#\# Random effects:

\#\# Formula: 1 | Site

\#\# (Intercept)

\#\# StdDev: 0.477398

\#\#

\#\# Formula: $\sim 1$ | Cluster \%in\% Site

\#\# (Intercept)

\#\# StdDev: 0.2130193

\#\#

\#\# Formula: 1 | Profile \%in\% Cluster \%in\% Site

\#\# (Intercept) Residual

\#\# StdDev: $1.820411 \mathrm{e}-050.4118728$ 


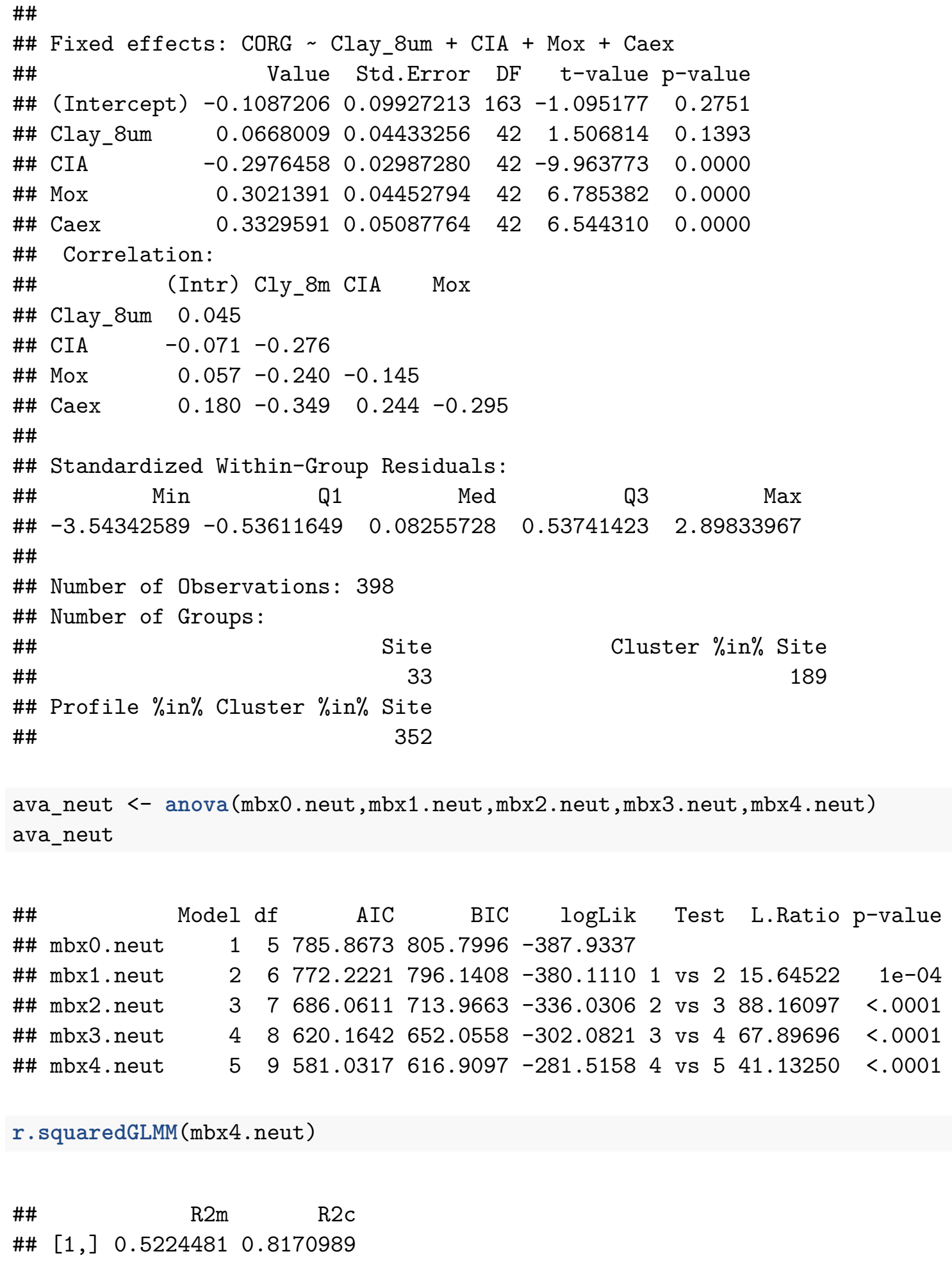

\section{Alkaline:}

Build models: 


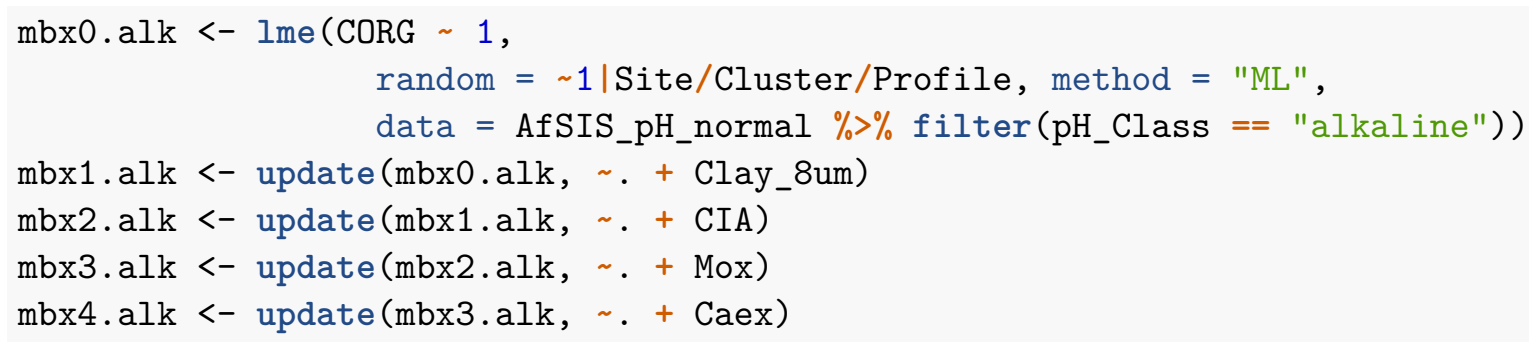

Autocorrelation:

vif_alk <- as.data.frame(vif (mbx4.alk))

vif_alk_test <- $\max \left(v i f \_a l k \$ " v i f(\operatorname{mbx} 4 . a l k) "\right)<3.0$

VIF < 3.0: TRUE -> no autocorrelation

Diagnostic plots:
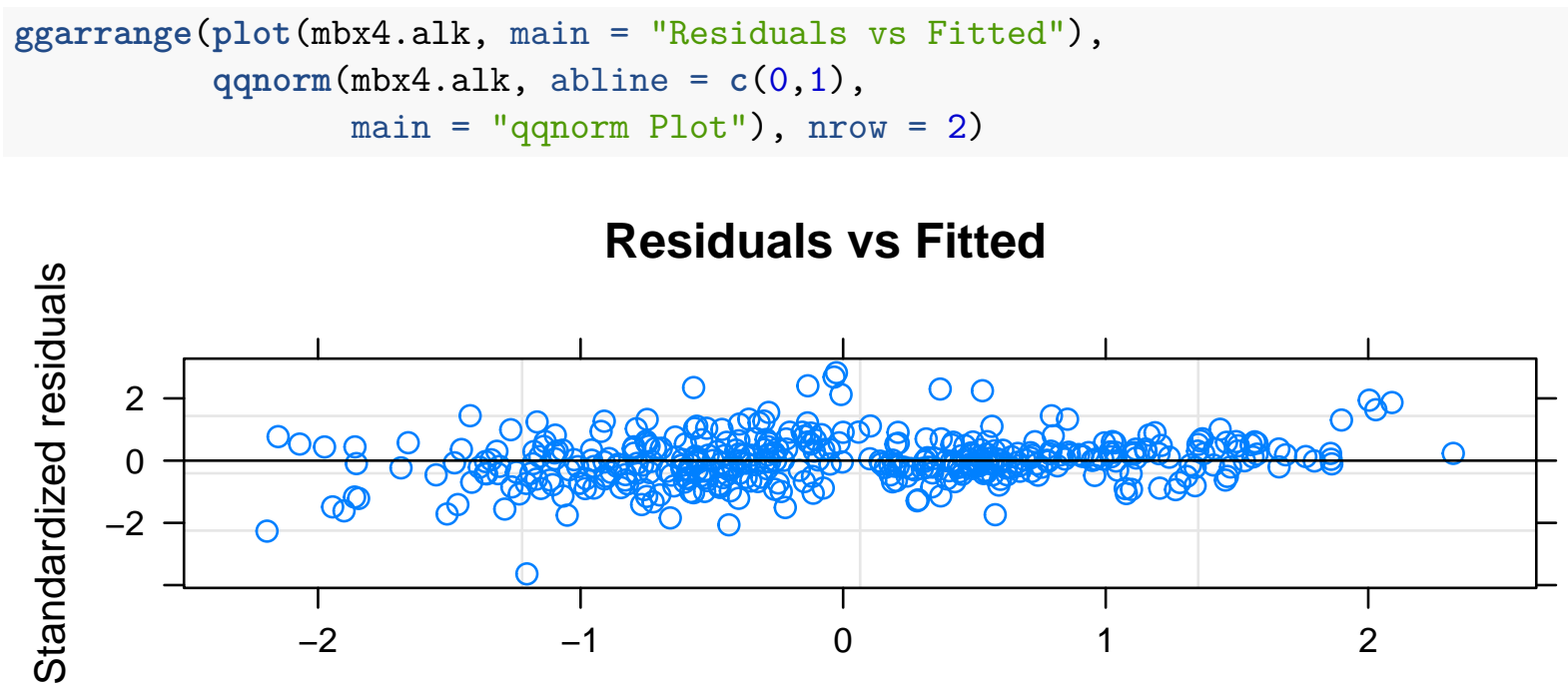

Fitted values

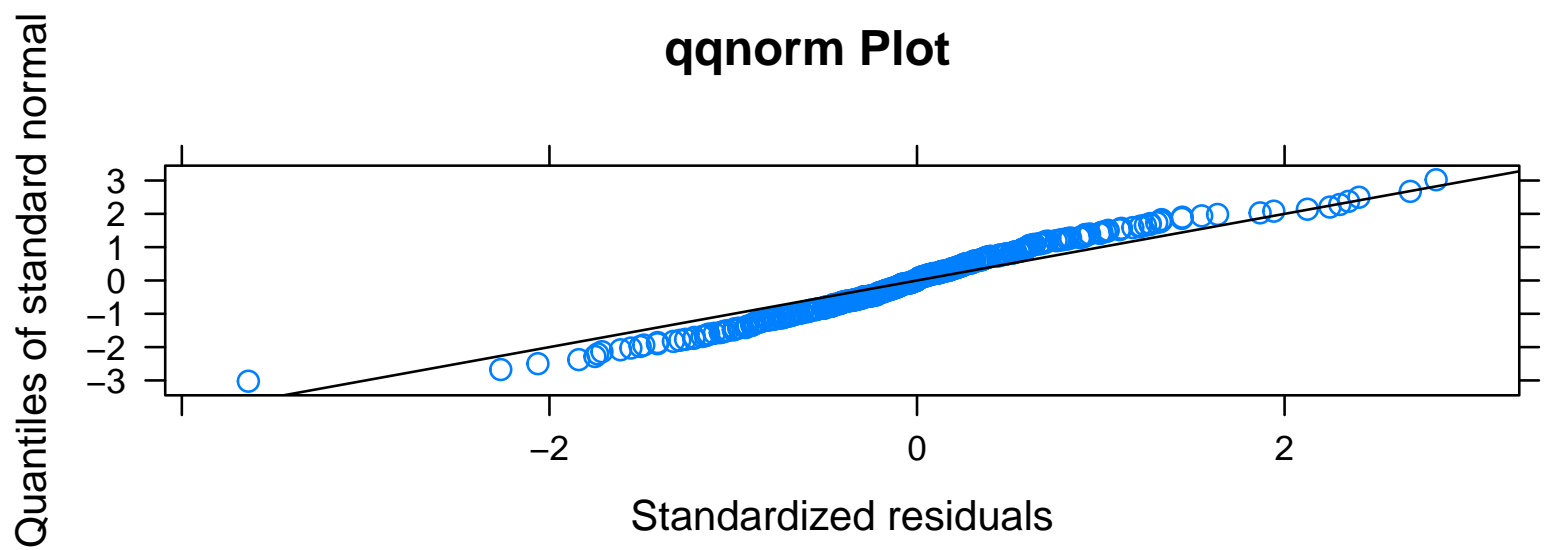

Model assumptions are met.

Anova output for all alkaline models (step-wise): 
$\operatorname{summary}(\operatorname{mbx} 4 . \mathrm{alk})$

\#\# Linear mixed-effects model fit by maximum likelihood

\#\# Data: AfSIS_pH_normal \% $\%$ filter(pH_Class == "alkaline")

\#\# AIC BIC logLik

\#\# $\quad 592.5831628 .5063 \quad-287.2915$

\#\#

\#\# Random effects:

\#\# Formula: 1 | Site

\#\# (Intercept)

\#\# StdDev: 0.595955

\#\#

\#\# Formula: $\sim 1$ | Cluster \%in\% Site

\#\# (Intercept)

\#\# StdDev: 0.1441046

\#\#

\#\# Formula: $~ 1$ | Profile \%in\% Cluster \%in\% Site

\#\# (Intercept) Residual

\#\# StdDev: 0.3173860 .3438606

\#\#

\#\# Fixed effects: CORG Clay_8um + CIA + Mox + Caex

\#\# Value Std.Error DF t-value p-value

$\begin{array}{llllll}\text { \#\# (Intercept) } & -0.0826417 & 0.16256046 & 221 & -0.508375 & 0.6117\end{array}$

$\begin{array}{llllll}\text { \#\# Clay_8um } & -0.1350680 & 0.03947549 & 76 & -3.421567 & 0.0010\end{array}$

$\begin{array}{llllll}\text { \#\# CIA } & -0.0458182 & 0.03441432 & 76 & -1.331372 & 0.1870\end{array}$

$\begin{array}{llllll}\text { \#\# Mox } & 0.1186411 & 0.03966816 & 76 & 2.990840 & 0.0037\end{array}$

$\begin{array}{lllllll}\text { \#\# Caex } & 0.3845744 & 0.04763336 & 76 & 8.073636 & 0.0000\end{array}$

\#\# Correlation:

\#\# (Intr) $\mathrm{Cly}_{-}$8m CIA Mox

\#\# Clay_8um 0.024

$\begin{array}{lll}\text { \#\# CIA } & -0.125 & -0.102\end{array}$

$\begin{array}{llll}\text { \#\# Mox } & 0.003 & -0.185 & 0.094\end{array}$

\#\# Caex $\quad 0.066-0.500-0.201-0.360$

\#\#

\#\# Standardized Within-Group Residuals:

\#\# $\quad$ Min $\quad$ Q1 $\quad$ Med $\quad$ Q3

$\begin{array}{llllll}\text { \#\# } & -3.638093299 & -0.411829300 & -0.005296146 & 0.370981139 & 2.824614787\end{array}$

\#\#

\#\# Number of Observations: 400

\#\# Number of Groups:

\#\# Site Cluster $\%$ in\% Site

\#\# 17

\#\# Profile \%in\% Cluster \%in\% Site

\#\#

320 


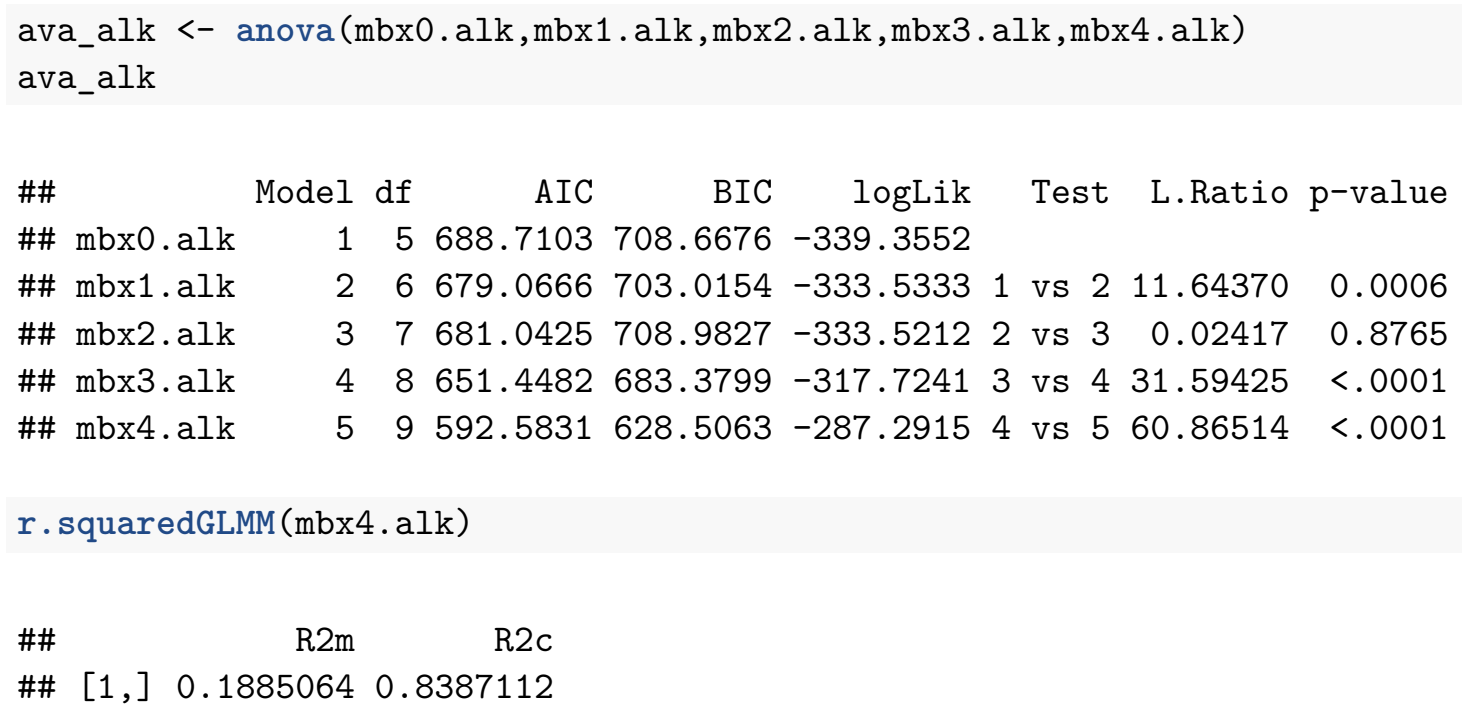

\section{Anova Summary table:}

Table $B 6$ in the supplement of the manuscript.

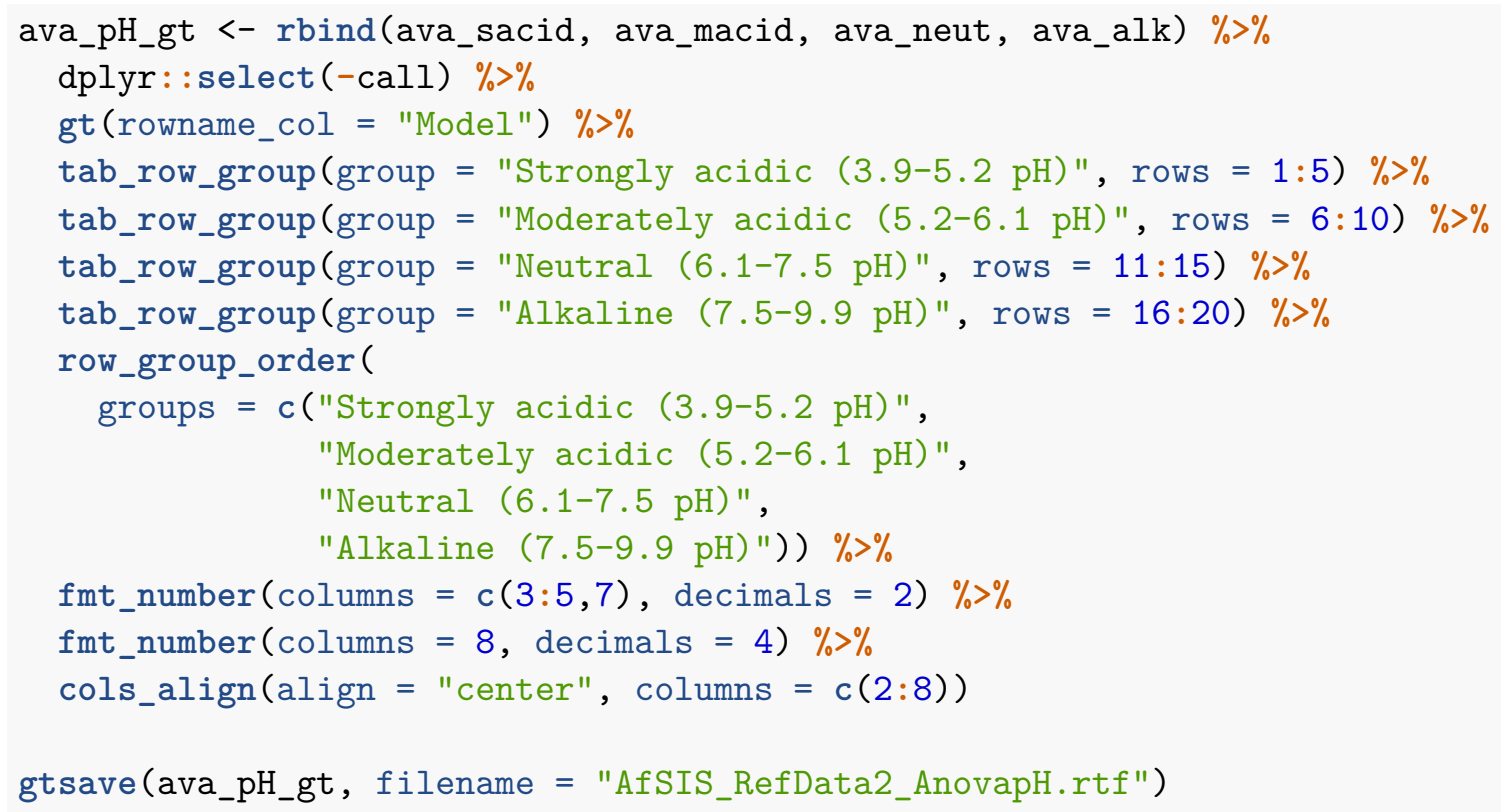

6.2.2.1 CIA models This section is providing the code for the moderately and highly weathered models, respectively.

\section{Normalize and standardize each sub-group:}

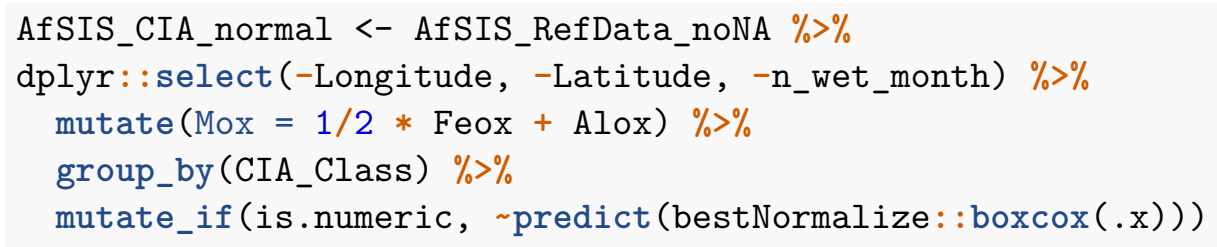




\section{Moderately weathered:}

\section{Build models:}

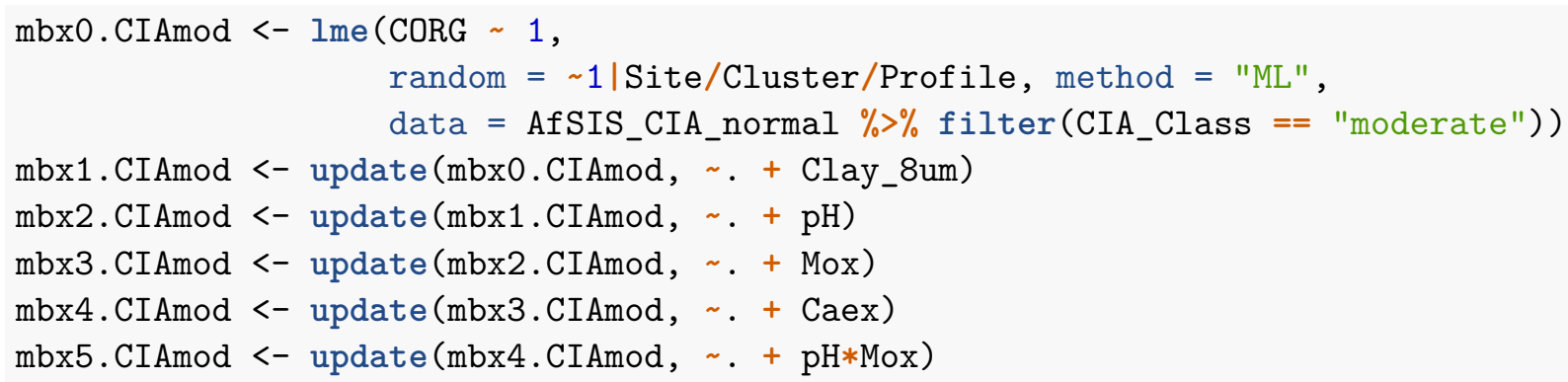

Autocorrelation:

vif_mod <- as.data.frame (vif (mbx5.CIAmod))

vif_mod_test <- $\max ($ vif_mod\$"vif (mbx5.CIAmod)" $)<3.0$

VIF $<3.0:$ TRUE -> no autocorrelation

Diagnostic plots:

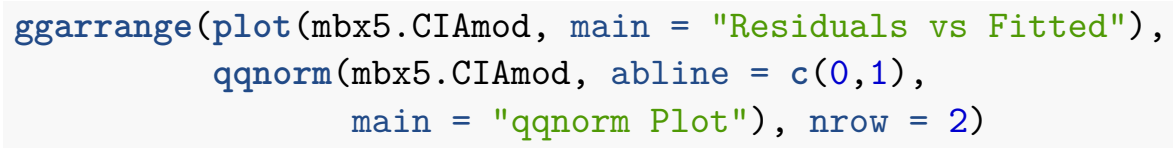

\section{Residuals vs Fitted}

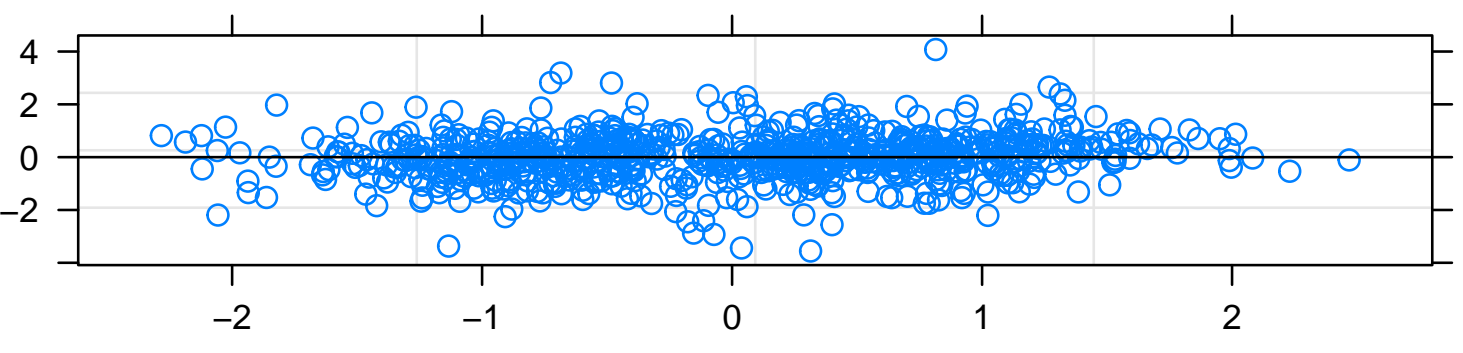

Fitted values

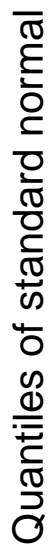

\section{qqnorm Plot}

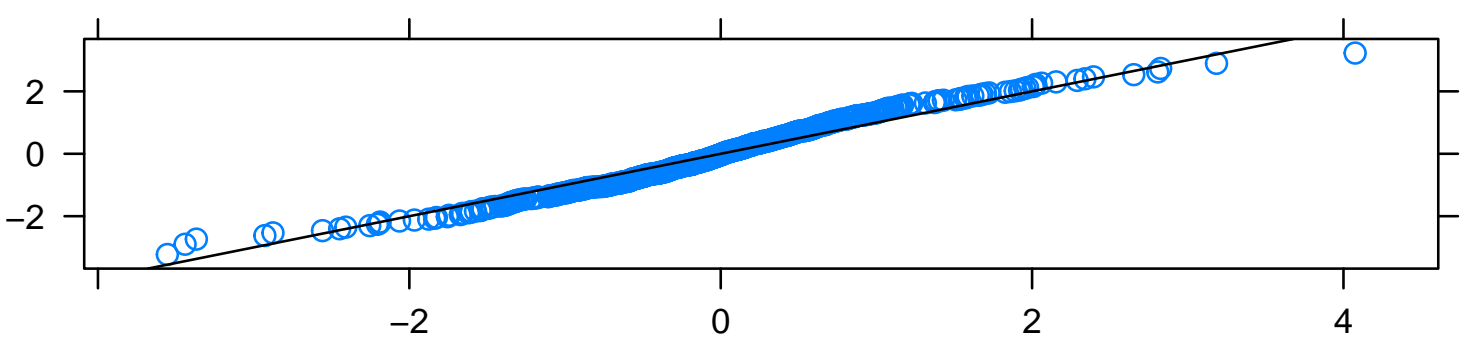

Standardized residuals 
Model assumptions are met.

Anova output for all moderately weathered models (step-wise):

summary (mbx5. CIAmod)

\#\# Linear mixed-effects model fit by maximum likelihood

\#\# Data: AfSIS_CIA_normal \% $\%$ filter(CIA_Class == "moderate")

\#\# AIC BIC logLik

\#\# $\quad 1151.672 \quad 1198.53-565.8358$

\#\#

\#\# Random effects:

\#\# Formula: 1 | Site

\#\# (Intercept)

\#\# StdDev: 0.4759728

\#\#

\#\# Formula: 1 | Cluster \%in\% Site

\#\# (Intercept)

\#\# StdDev: 0.1941322

\#\#

\#\# Formula: 1 | Profile \%in\% Cluster \%in $\%$ Site

\#\# (Intercept) Residual

\#\# StdDev: 0.191248 0.3958591

\#\#

\#\# Fixed effects: CORG Clay_8um + pH + Mox + Caex + pH:Mox

\#\# Value Std.Error DF t-value p-value

$\begin{array}{llllll}\text { \#\# (Intercept) } & -0.0577251 & 0.09352627 & 429 & -0.617207 & 0.5374\end{array}$

\#\# Clay_8um $\quad-0.1262774 \quad 0.02949055 \quad 121 \quad-4.281961 \quad 0.0000$

\#\# pH $\quad-0.3048058 \quad 0.03623837 \quad 121 \quad-8.411136 \quad 0.0000$

$\begin{array}{lllllll}\text { \#\# Mox } & 0.1752278 & 0.03131202 & 121 & 5.596184 & 0.0000\end{array}$

$\begin{array}{llllll}\text { \#\# Caex } & 0.5624563 & 0.03758580 & 121 & 14.964595 & 0.0000\end{array}$

$\begin{array}{lllllll}\text { \#\# } \mathrm{pH}: \text { Mox } & -0.0860093 & 0.01935993 & 121 & -4.442646 & 0.0000\end{array}$

\#\# Correlation:

\#\# (Intr) $\mathrm{Cly}_{-} 8 \mathrm{~m} \mathrm{pH} \quad$ Mox Caex

\#\# Clay_8um 0.042

\#\# $\mathrm{pH} \quad 0.126 \quad 0.053$

$\begin{array}{llll}\text { \#\# Mox } & 0.013 & -0.209 & 0.216\end{array}$

\#\# Caex $\quad 0.101-0.435-0.323-0.398$

$\begin{array}{llllll}\text { \#\# } \mathrm{pH}: \operatorname{Mox} & -0.015 & -0.097 & 0.198 & 0.291 & -0.099\end{array}$

\#\#

\#\# Standardized Within-Group Residuals:

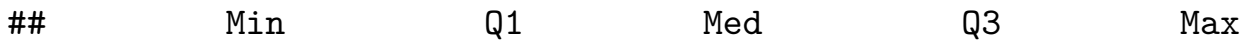

$\begin{array}{llllll}\text { \#\# } & -3.55417923 & -0.46816528 & 0.01375396 & 0.47170274 & 4.07478271\end{array}$

\#\#

\#\# Number of Observations: 801

\#\# Number of Groups:

\#\#

Site

Cluster $\%$ in $\%$ Site

\#\#

35

246 
\#\# Profile \%in\% Cluster \%in\% Site

\#\#

675

ava_CIAmod <- anova (mbx0.CIAmod,mbx1.CIAmod,mbx2.CIAmod,mbx3.CIAmod, mbx4.CIAmod, mbx5.CIAmod) ava_CIAmod

\begin{tabular}{|c|c|c|c|c|c|c|c|c|}
\hline \#\# & Model & & AIC & BIC & logLik & Test & L.Ratio & p-value \\
\hline \#\# mbx0.CIAmod & 1 & 5 & 1535.350 & 8.779 & -762.6749 & & & \\
\hline$T$ & 2 & 6 & .429 & 523.544 & -741.7144 & $1 \mathrm{vs} 2$ & 92114 & \\
\hline & 3 & 7 & 1487.129 & 1519.930 & -736.5645 & 2 vs 3 & 9961 & \\
\hline & 4 & 8 & 94 & 1390 & -668.3468 & 3 vs 4 & 43540 & \\
\hline & 5 & 9 & 1169.173 & 1211.346 & -575.5865 & 4 vs 5 & 185.52061 & \\
\hline bx5. CIAmod & 6 & & 1151.671 & 1198.530 & -565.8358 & 5 vs 6 & 19.50155 & $<.00$ \\
\hline
\end{tabular}

r.squaredGLMM (mbx5.CIAmod)

\#\# $\quad$ R2m $\quad$ R2c

\#\# [1,] 0.4342010 .8062081

\section{Higly weathered:}

Build models:

mbx0.CIAhigh $<-$ Ime $($ CORG $\sim 1$, random $=\sim 1 \mid$ Site/Cluster $/$ Profile, method = "ML", data $=$ AfSIS_CIA_normal $\%$ filter $($ CIA_Class == "high"))

mbx1.CIAhigh <- update (mbx0.CIAhigh, . + Clay_8um)

mbx2.CIAhigh <- update (mbx1.CIAhigh, . + $\mathrm{pH})$

mbx3.CIAhigh <- update (mbx2.CIAhigh, . + Mox)

mbx4.CIAhigh <- update (mbx3.CIAhigh, . + Caex)

mbx5.CIAhigh <- update (mbx4.CIAhigh, . + $\mathrm{pH} *$ Mox $)$

\section{Autocorrelation:}

vif_high <- as.data.frame (vif(mbx5.CIAhigh))

vif_high_test <- $\max \left(v i f \_h i g h \$ " v i f(\operatorname{mbx} 5\right.$. CIAhigh)" $)<3.0$

VIF < 3.0: TRUE -> no autocorrelation

Diagnostic plots:

ggarrange(plot(mbx5.CIAhigh, main = "Residuals vs Fitted"), qqnorm(mbx5. CIAhigh, abline $=c(0,1)$, main = "qqnorm Plot"), nrow = 2) 


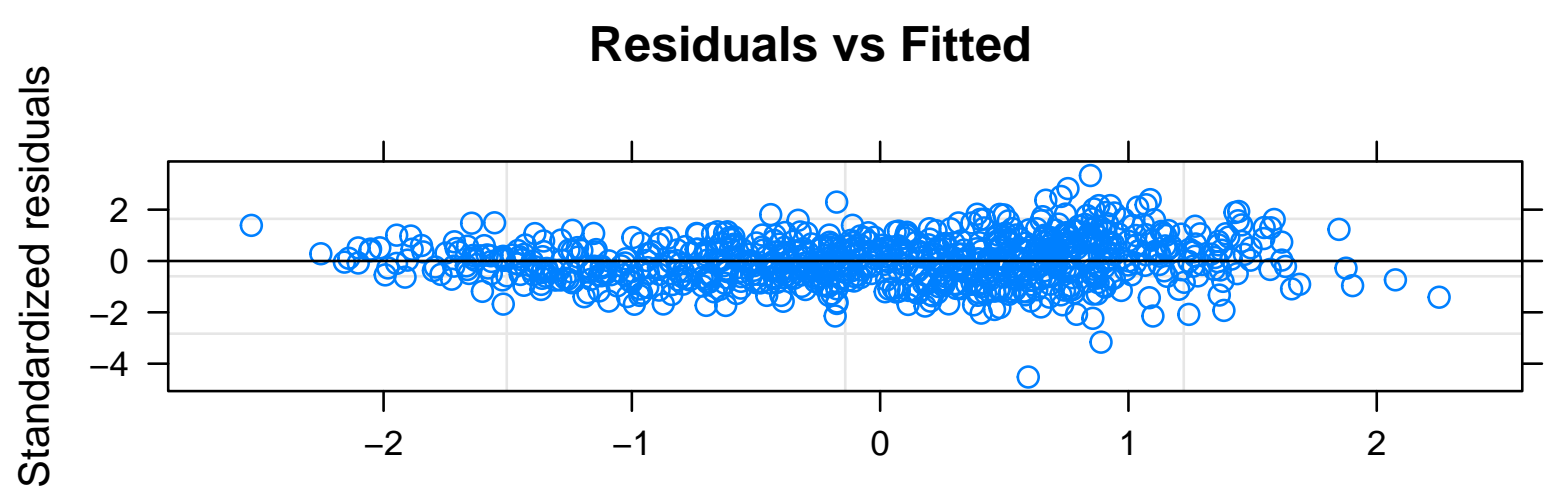

Fitted values

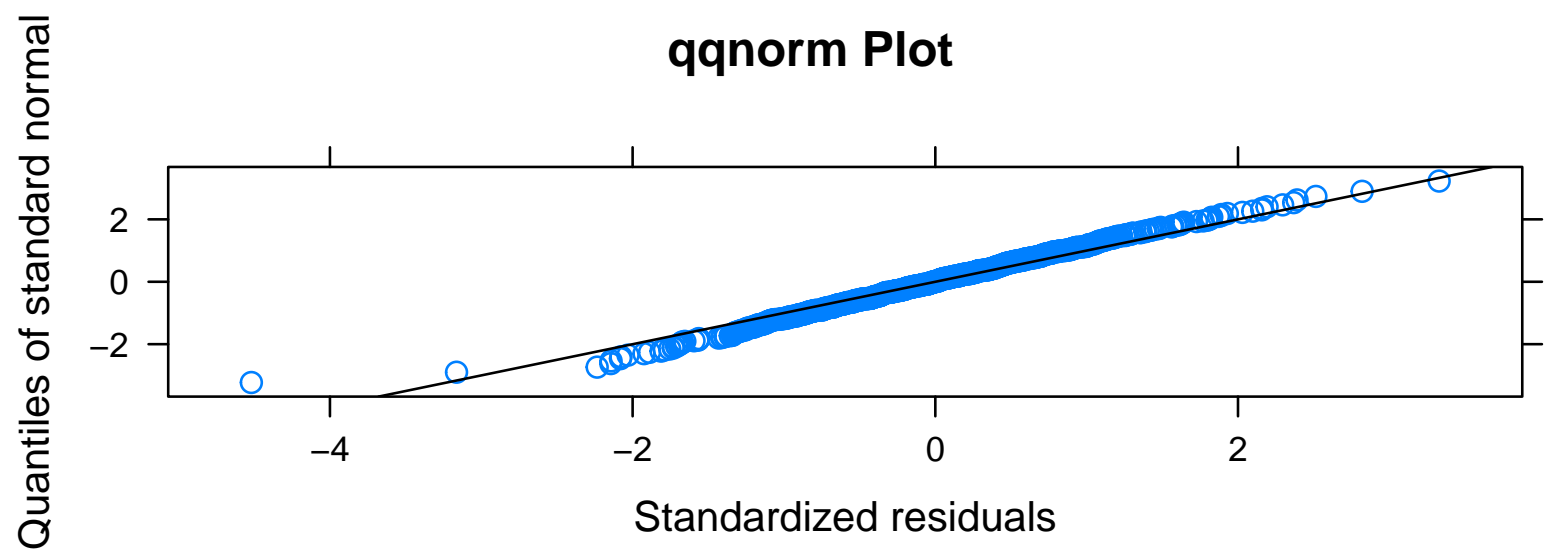

Model assumptions are met.

Anova output for all highly weathered models (step-wise):

summary (mbx5. CIAhigh)

\#\# Linear mixed-effects model fit by maximum likelihood

\#\# Data: AfSIS_CIA_normal \% $\%$ filter(CIA_Class == "high")

\#\# AIC BIC logLik

$\begin{array}{llll}\text { \#\# } & 1215.271 & 1262.117 & -597.6357\end{array}$

\#\#

\#\# Random effects:

\#\# Formula: 1 | Site

\#\# (Intercept)

\#\# StdDev: 0.5005484

\#\#

\#\# Formula: $~ 1$ | Cluster \%in\% Site

\#\# (Intercept)

\#\# StdDev: 0.2307733

\#\#

\#\# Formula: 1 | Profile \%in\% Cluster \%in\% Site

\#\# (Intercept) Residual

\#\# StdDev: 0.15921320 .4173483 


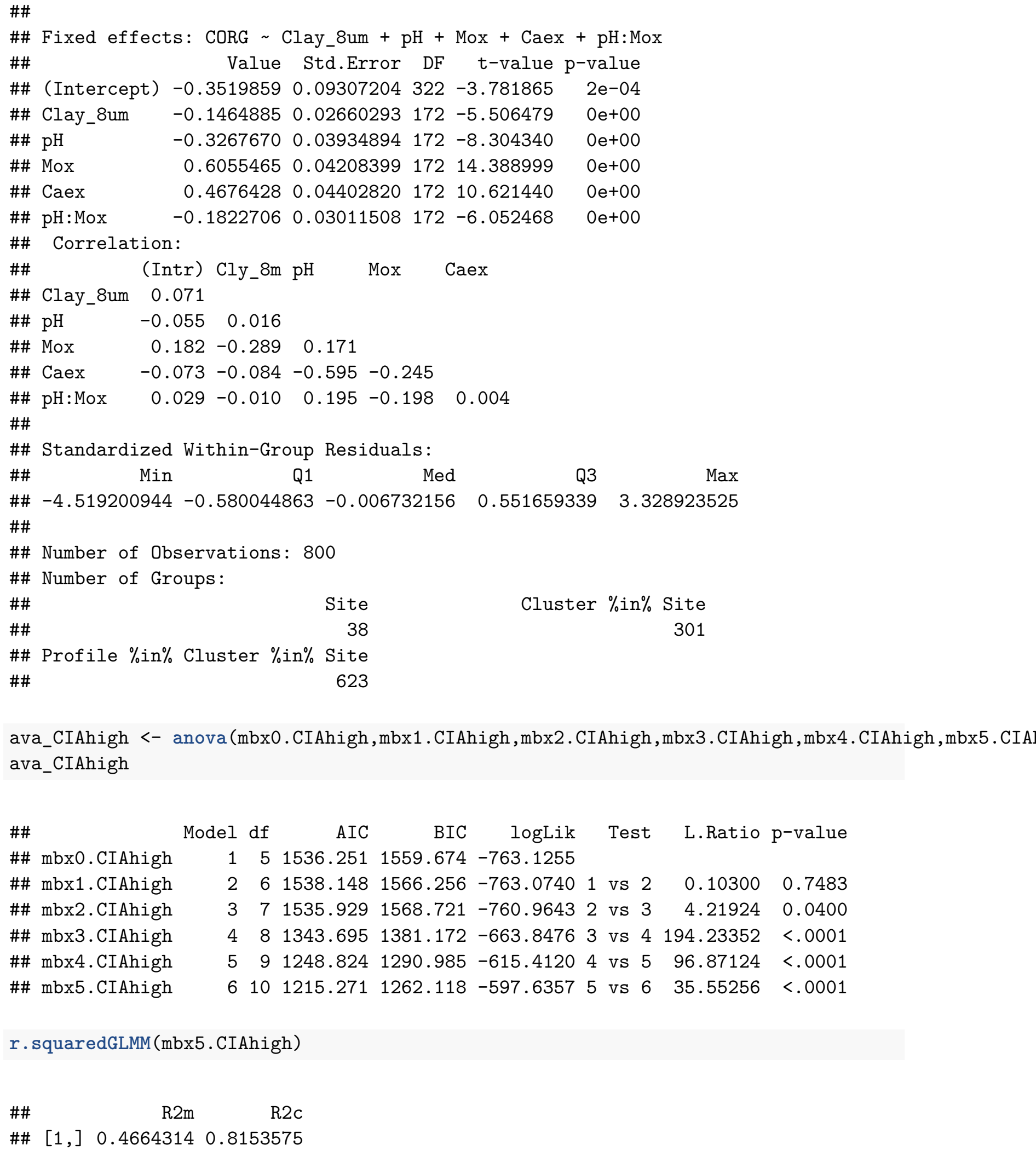




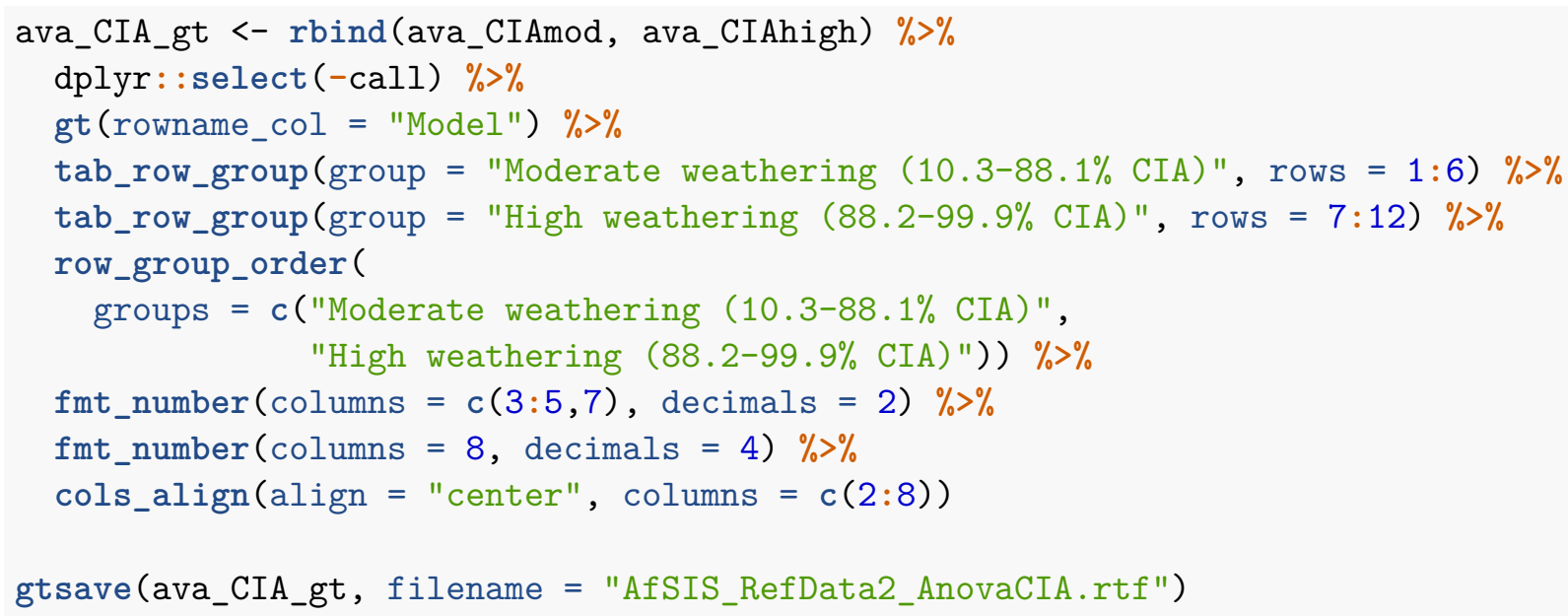

\subsubsection{Seasonality models}

This section provides all the code for the modelling of the three seasonality groups $(0,1-3$, and $4-7$ number of wet months).

\section{Normalize and standardize by sub-groups:}

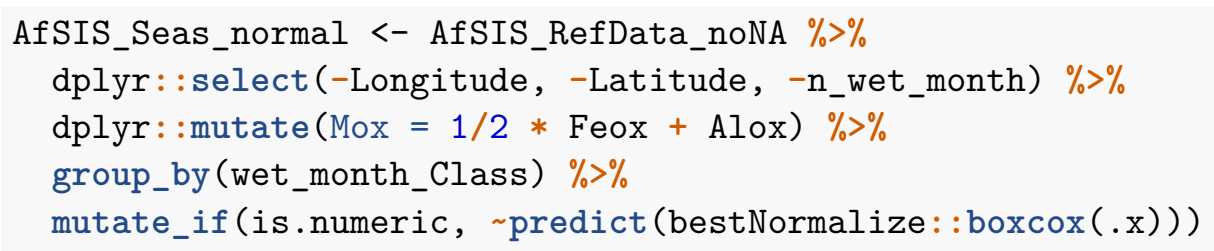

\section{0 number of wet months:}

Build models:

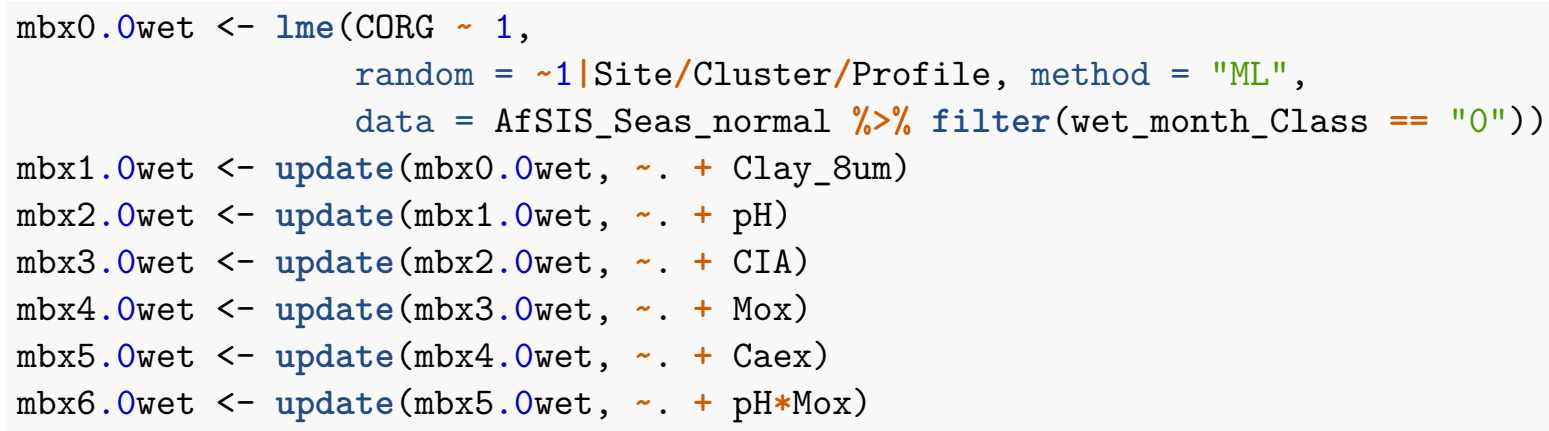

Autocorrelation:

vif_Owet <- as.data.frame (vif (mbx6.0wet))

vif_Owet_test <- $\max ($ vif_Owet\$"vif(mbx6.0wet)" $)<3.0$ 
VIF < 3.0: TRUE -> no autocorrelation

Diagnostic plots:
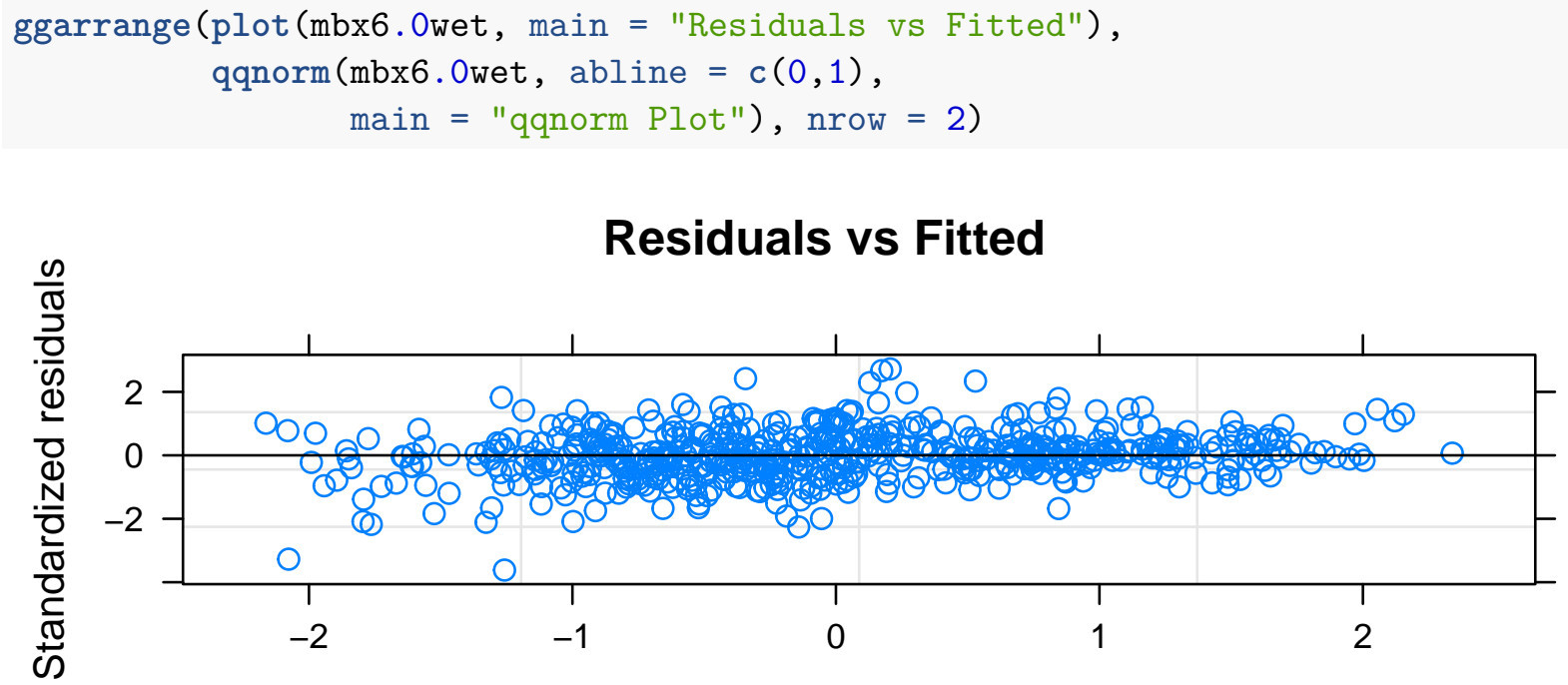

Fitted values

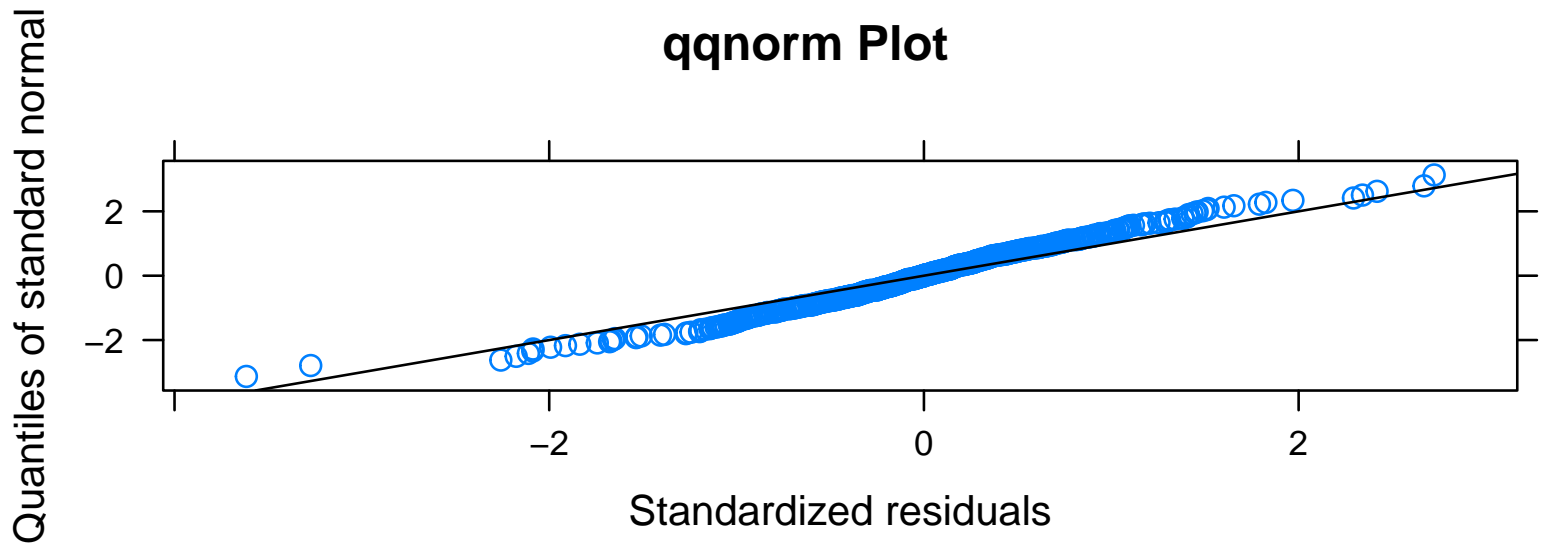

Model assumptions are met.

Anova output for all 0 number of wet months models (step-wise):

summary (mbx6. Owet)

\#\# Linear mixed-effects model fit by maximum likelihood

\#\# Data: AfSIS_Seas_normal \% $\%$ filter (wet_month_Class == "0")

\#\# AIC BIC logLik

\#\# $\quad 840.0764 \quad 887.9169 \quad-409.0382$

\#\#

\#\# Random effects:

\#\# Formula: 1 | Site

\#\# (Intercept)

\#\# StdDev: 0.5621932

\#\# 


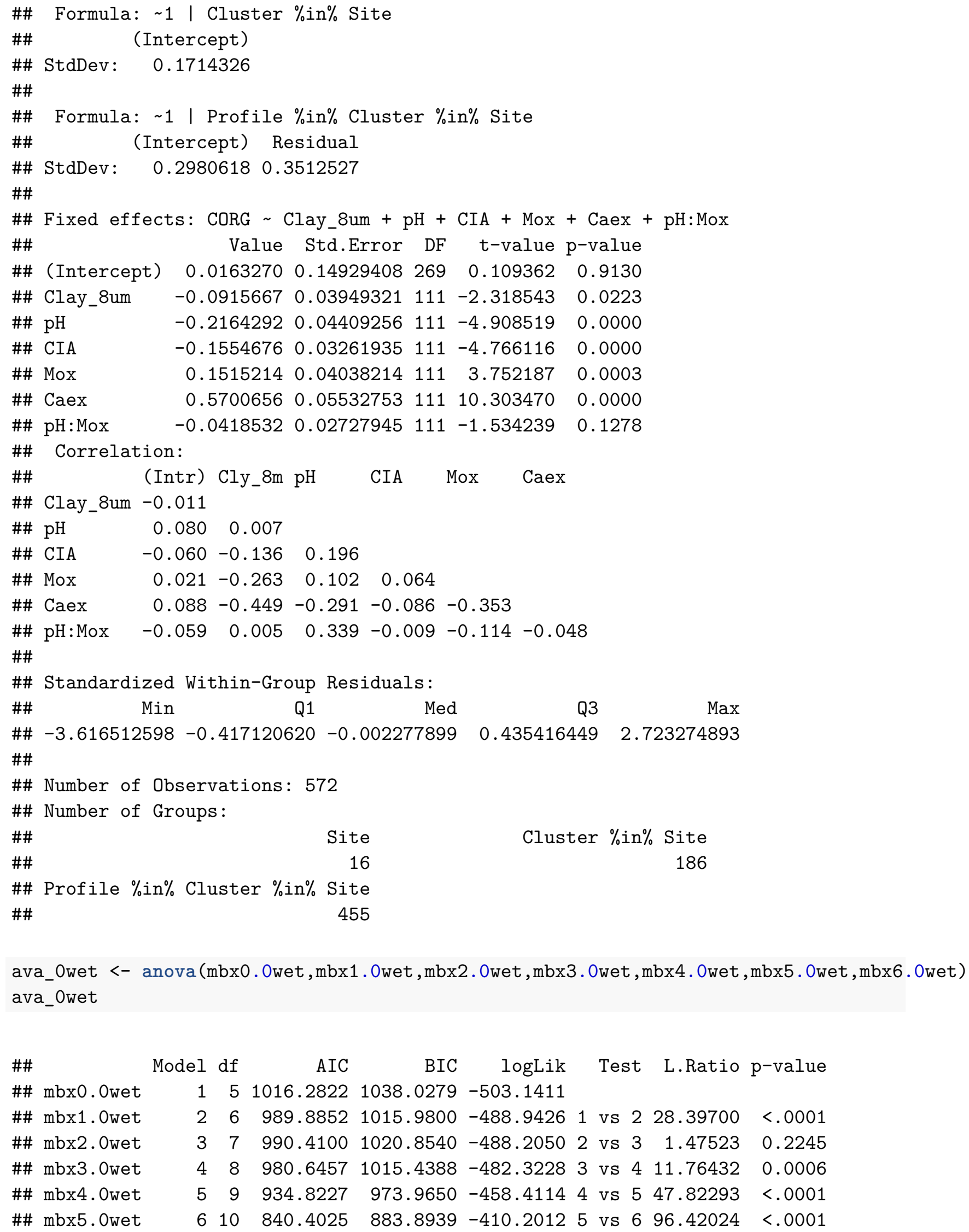


r.squaredGLMM (mbx5. 0wet)

\#\# $\quad$ R2m $\quad$ R2c

\#\# [1, ] $0.330346 \quad 0.8595547$

\section{1-3 number of wet months:}

Build models:

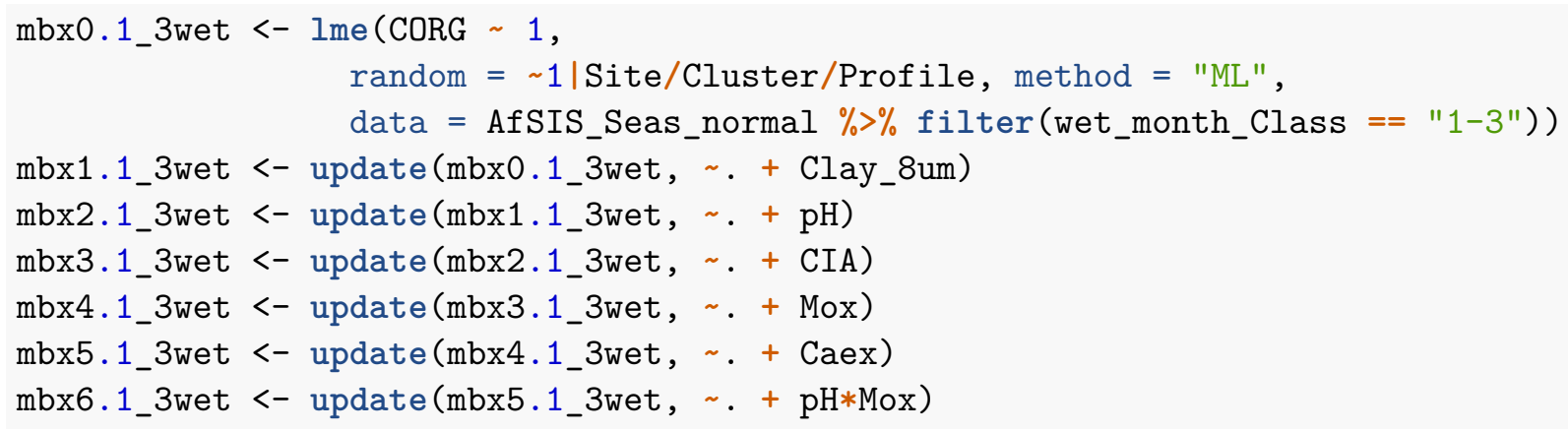

Autocorrelation:

vif_1_3wet <- as.data.frame (vif (mbx6.1_3wet))

vif_1_3wet_test <- $\max ($ vif_1_3wet $\$ " v i f(\operatorname{mbx} 6.1$ _3wet $) ")<3.0$

VIF < 3.0: TRUE -> no autocorrelation

Diagnostic plots:

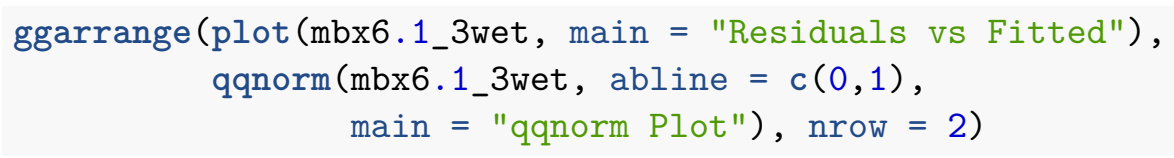




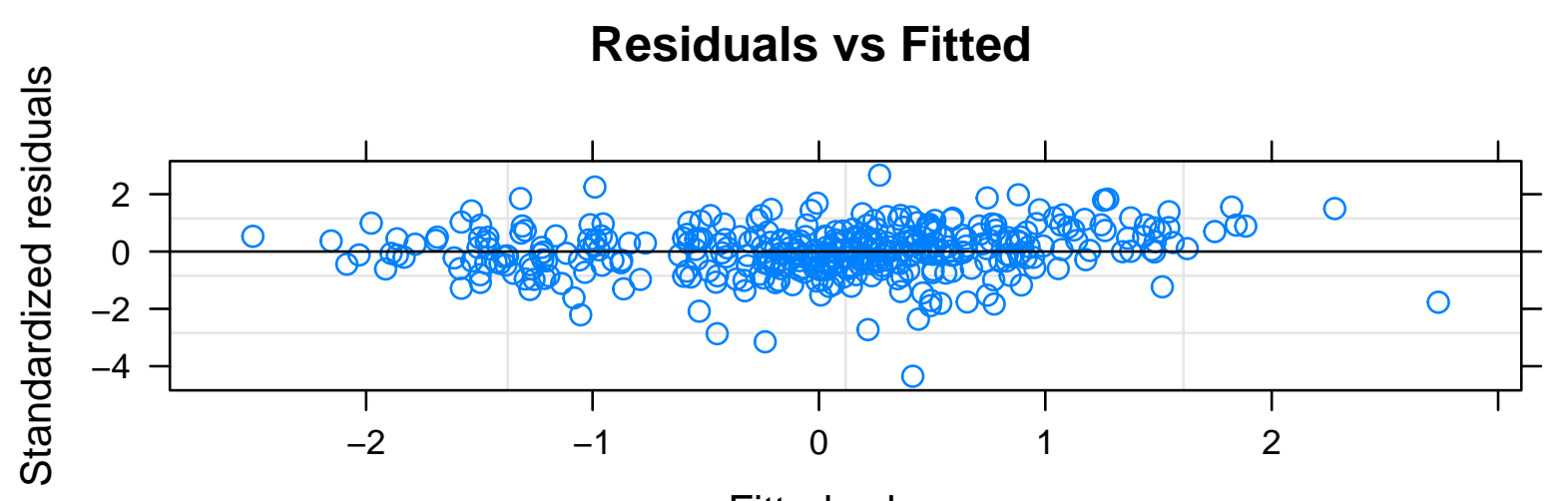

Fitted values

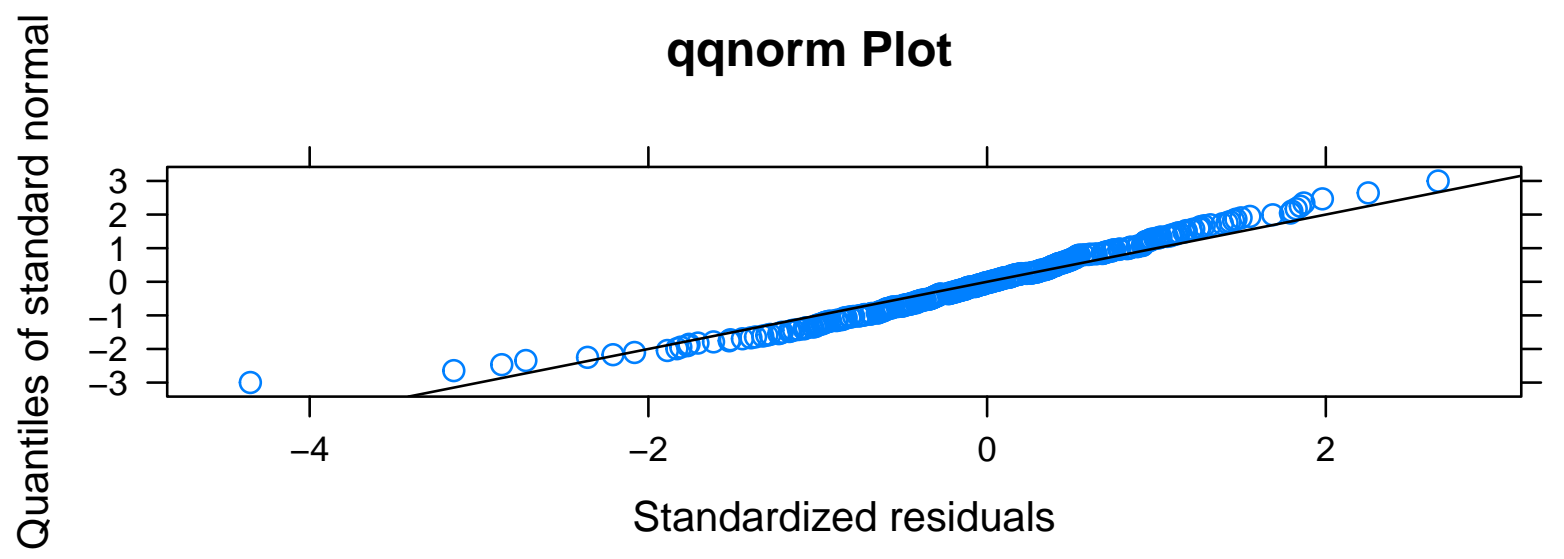

Model assumptions are met.

Anova output for all 1-3 number of wet months models (step-wise):

summary (mbx6.1_3wet)

\#\# Linear mixed-effects model fit by maximum likelihood

\#\# Data: AfSIS_Seas_normal \% $\%$ filter (wet_month_Class == "1-3")

\#\# AIC BIC logLik

\#\# $\quad 599.7013 \quad 642.6603-288.8506$

\#\#

\#\# Random effects:

\#\# Formula: 1 | Site

\#\# (Intercept)

\#\# StdDev: 0.4256601

\#\#

\#\# Formula: 1 | Cluster \%in\% Site

\#\# (Intercept)

\#\# StdDev: 0.2880689

\#\#

\#\# Formula: 1 | Profile $\%$ in $\%$ Cluster $\%$ in $\%$ Site

\#\# (Intercept) Residual

\#\# StdDev: 0.18067910 .4273154 


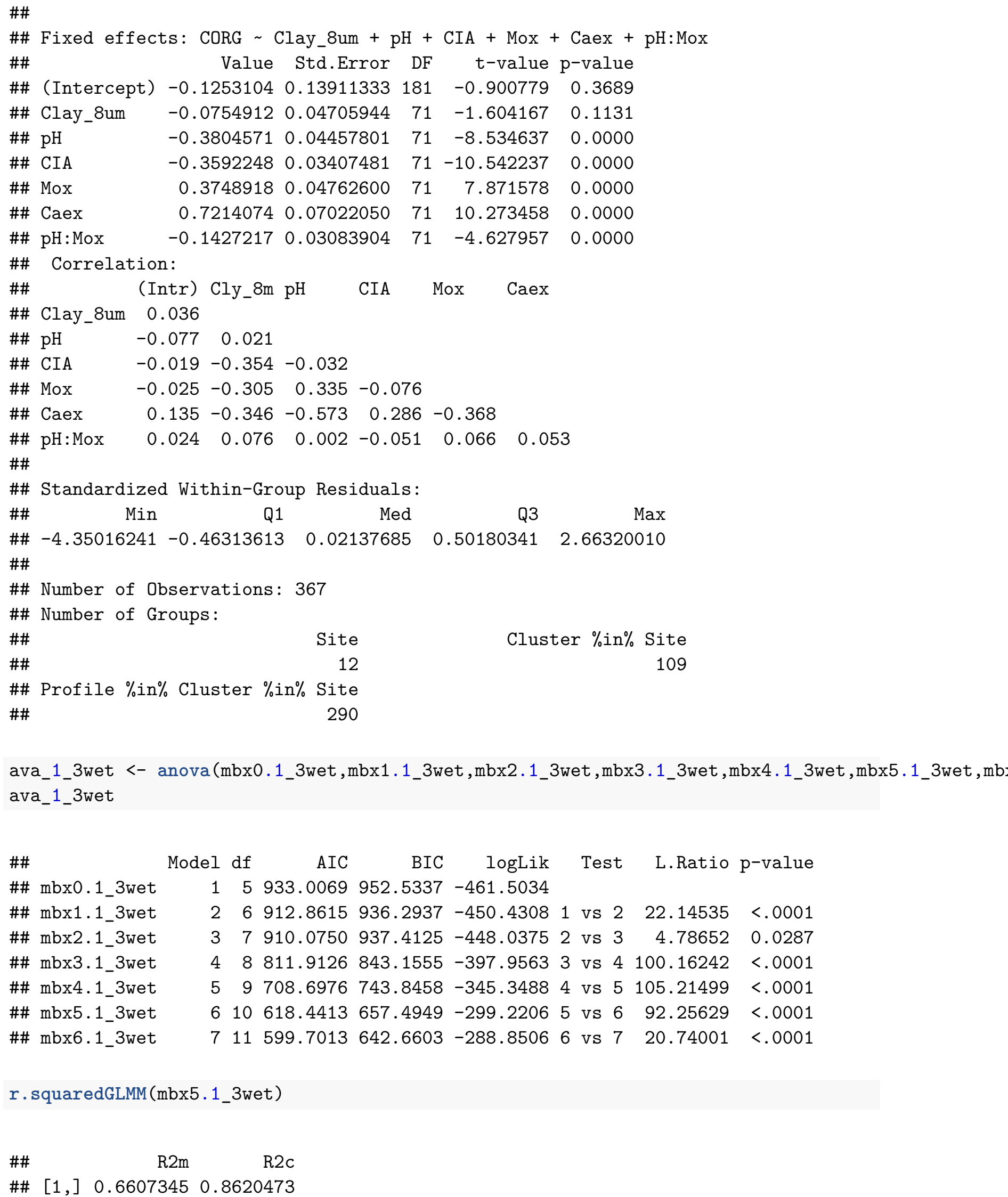




\section{4-7 number of wet months:}

Build models:

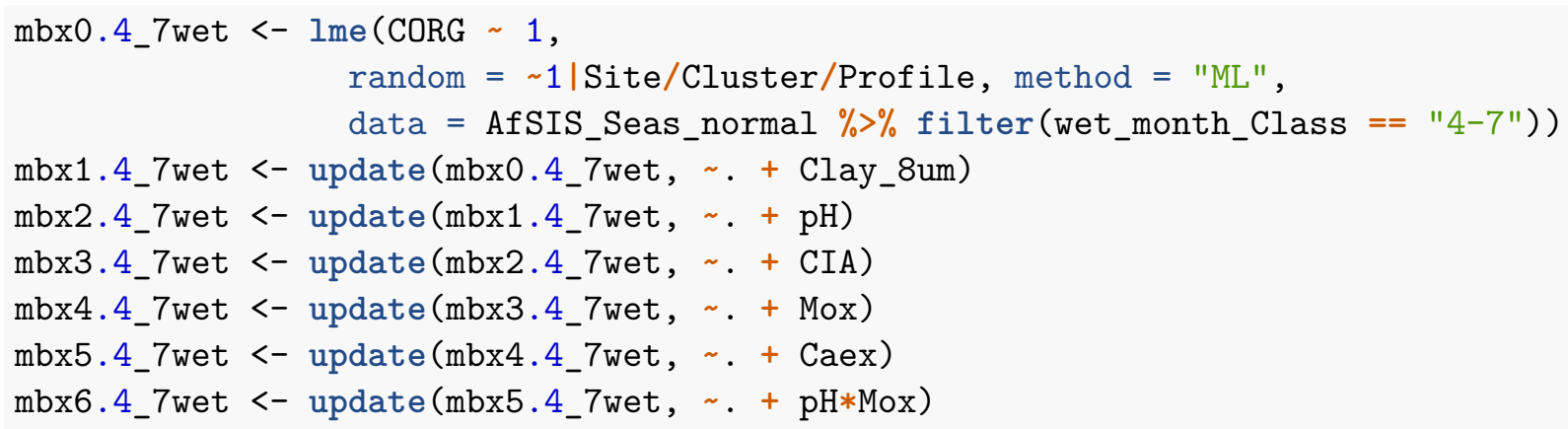

Autocorrelation:

vif_4_7wet <- as.data.frame (vif (mbx6.4_7wet))

vif_4_7wet_test <- $\max ($ vif_4_7wet $\$ " \operatorname{vif}(\operatorname{mbx} 6.4$ _ 7 wet $) ")<3.0$

VIF < 3.0: TRUE -> no autocorrelation

Diagnostic plots:

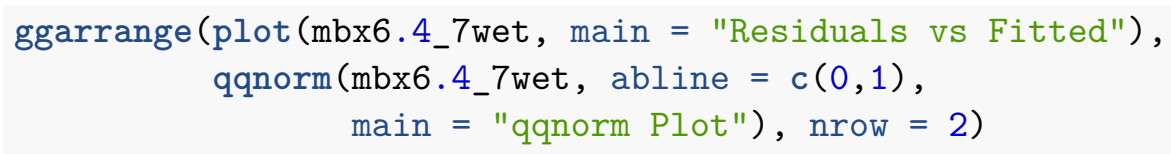



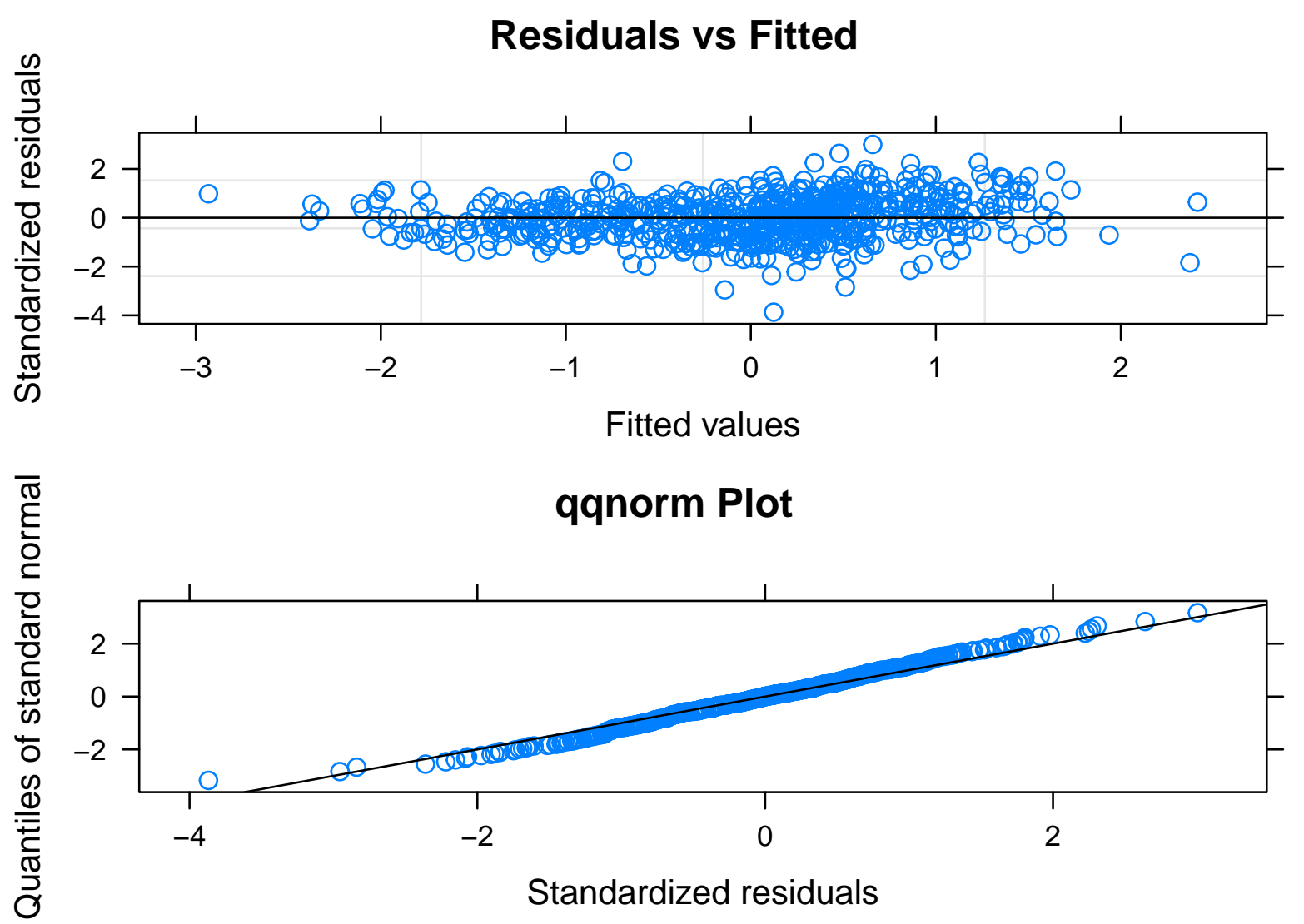

Model assumptions are met.

Anova output for all 4-7 number of wet months models (step-wise):

$\operatorname{summary}\left(\operatorname{mbx} 6.4 \_7 \mathrm{wet}\right)$

\#\# Linear mixed-effects model fit by maximum likelihood

\#\# Data: AfSIS_Seas_normal $\%$ filter (wet_month_Class == "4-7")

\#\# $\quad$ AIC $\quad$ BIC logLik

$\begin{array}{llll}\text { \#\# } \quad 1237.137 & 1286.585 & -607.5683\end{array}$

\#\#

\#\# Random effects:

\#\# Formula: 1 | Site

\#\# (Intercept)

\#\# StdDev: 0.4988179

\#\#

\#\# Formula: $\sim 1$ | Cluster \%in\% Site

\#\# (Intercept)

\#\# StdDev: 0.3261345

\#\#

\#\# Formula: $~ 1$ | Profile \%in\% Cluster \%in\% Site

\#\# (Intercept) Residual

$\begin{array}{lll}\text { \#\# StdDev: } 0.2217827 & 0.4942388\end{array}$ 


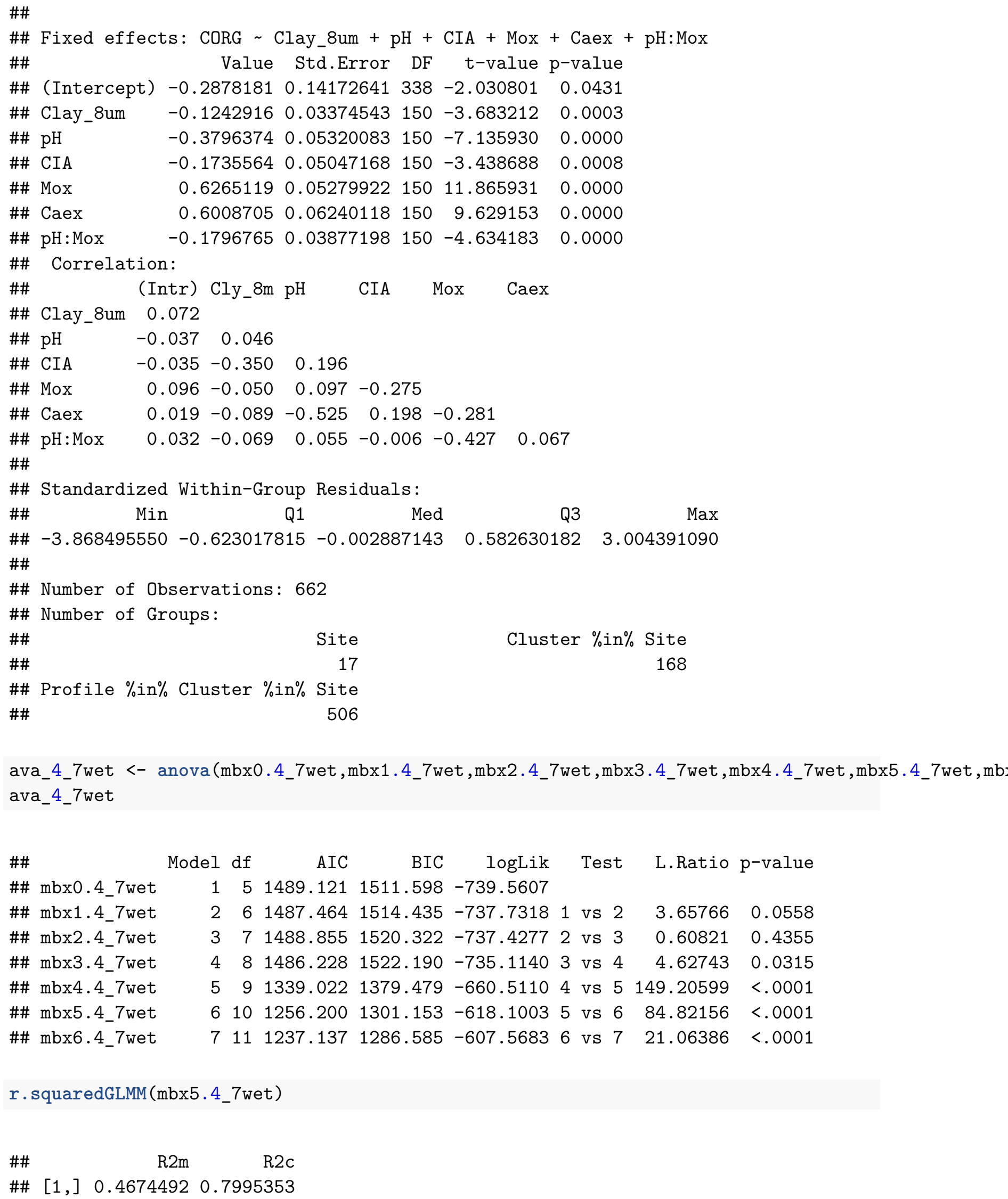




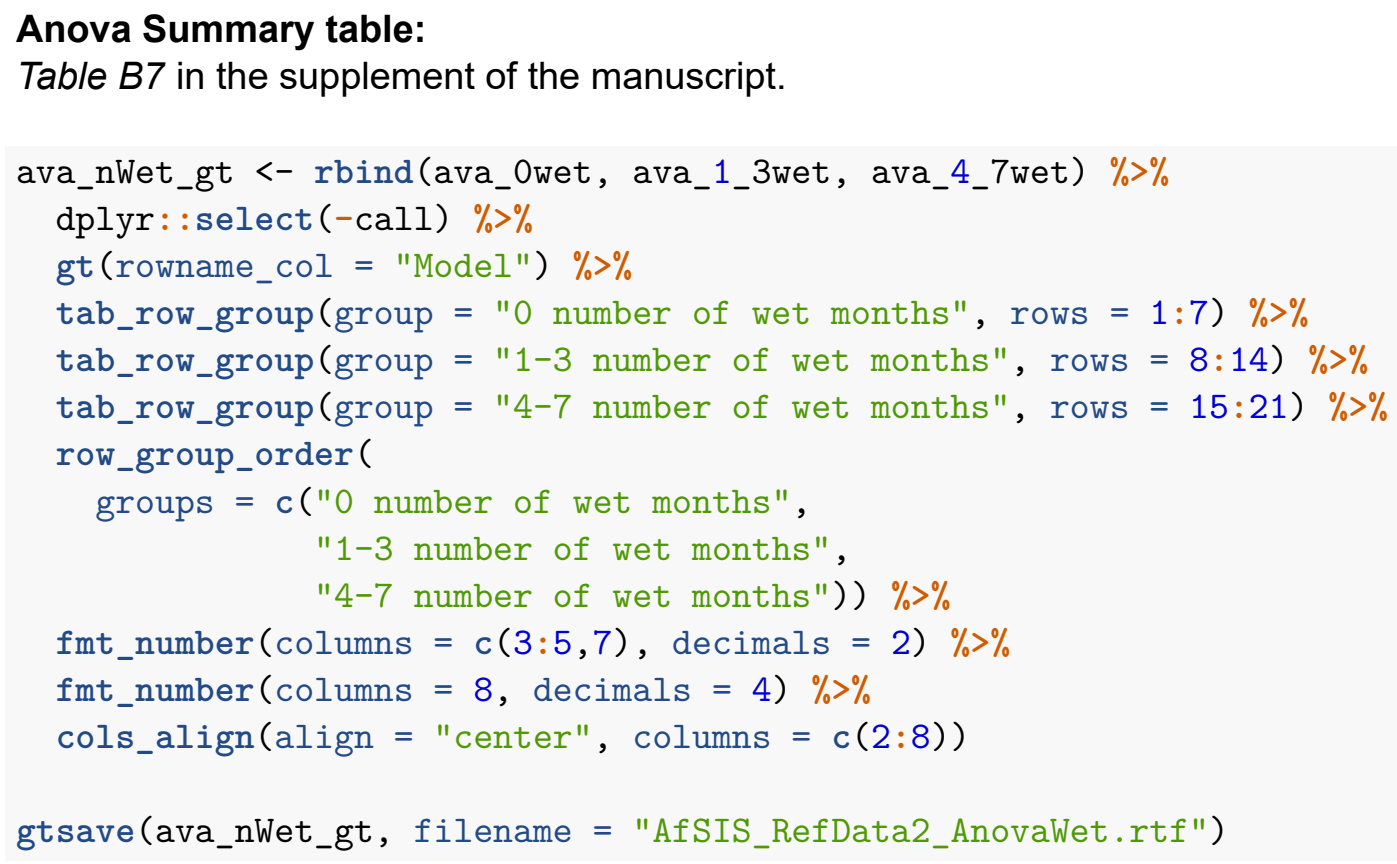

\subsubsection{Land cover models}

This section contains the four models for the forest, cropland, grassland and other samples, respectively.

\section{Normalize and standardize each sub-group:}

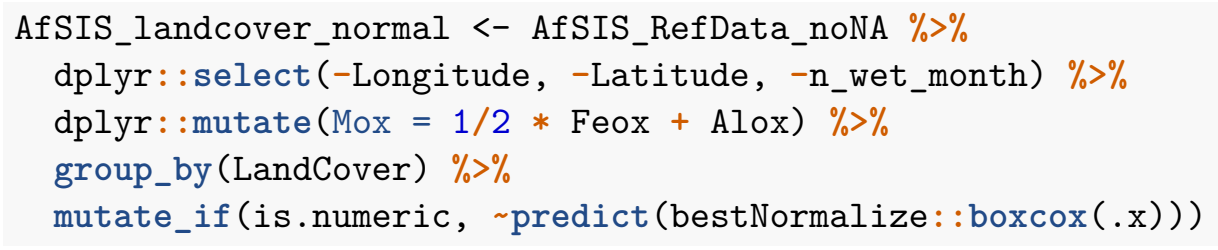

\section{Cropland:}

Build models:

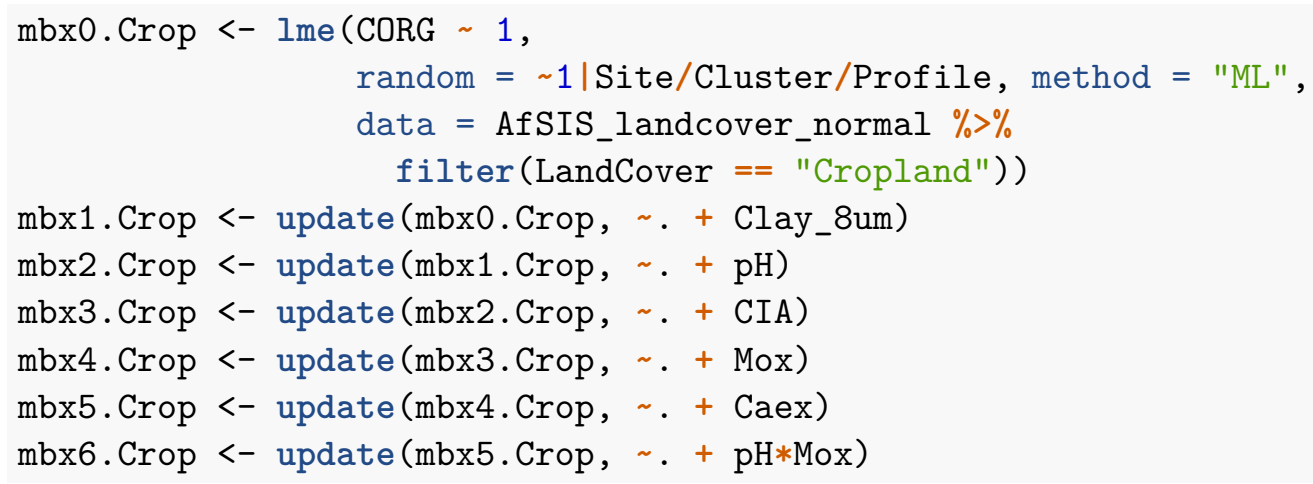


Autocorrelation:

vif_Crop <- as.data.frame (vif (mbx6.Crop))

vif_Crop_test <- $\max \left(v i f \_C r o p \$ " v i f(m b x 6 . C r o p) "\right)<3.0$

VIF < 3.0: TRUE -> no autocorrelation

Diagnostic plots:
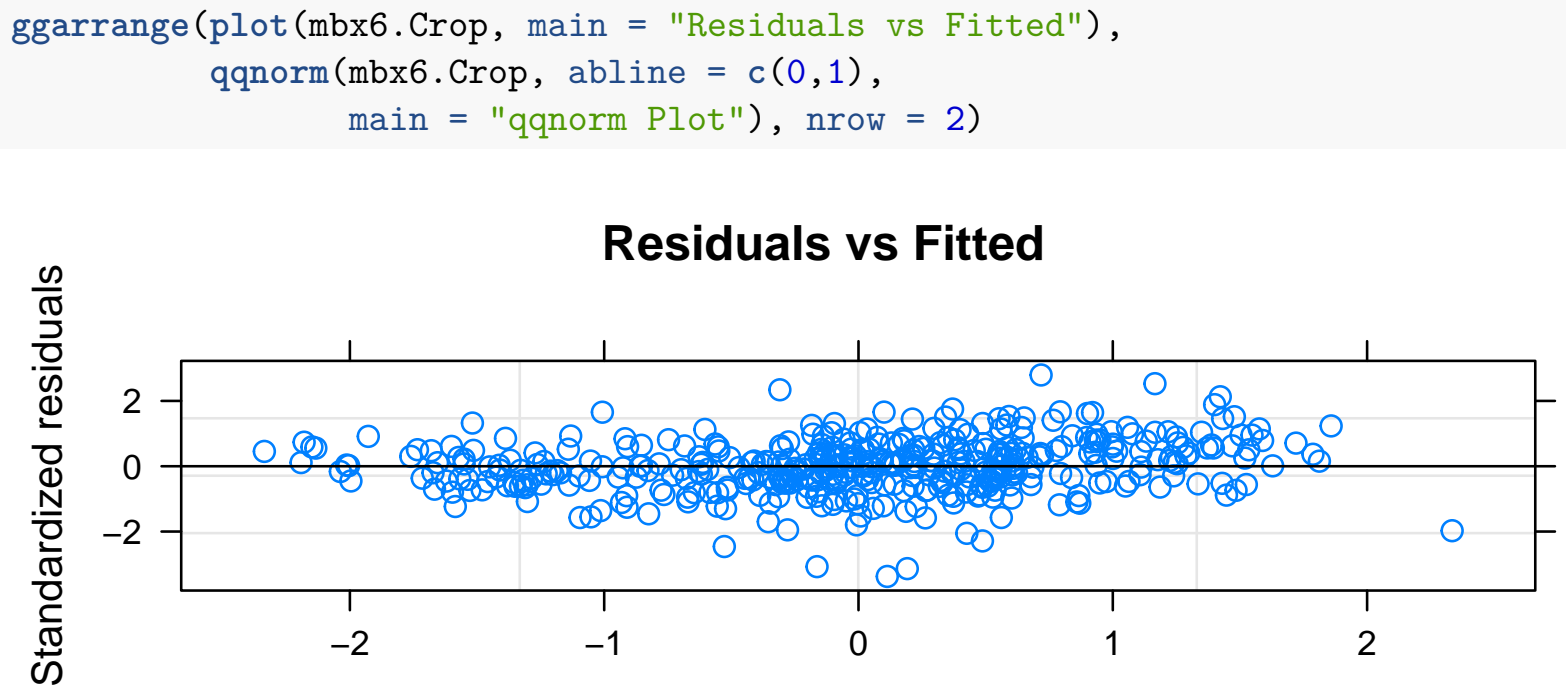

Fitted values

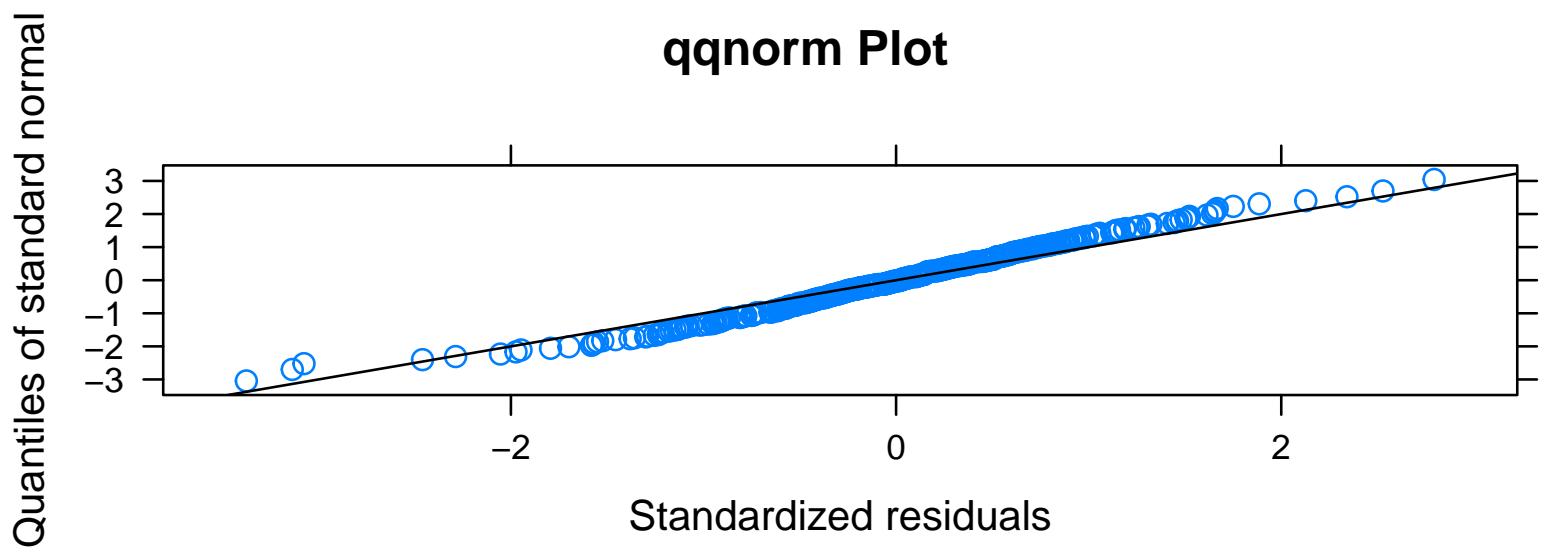

Model assumptions are met.

Anova output for all cropland models (step-wise):

summary (mbx6. Crop)

\#\# Linear mixed-effects model fit by maximum likelihood

\#\# Data: AfSIS_landcover_normal \% $\%$ filter(LandCover == "Cropland")

\#\# AIC BIC logLik

\#\# $\quad 736.8044 \quad 781.4804-357.4022$ 


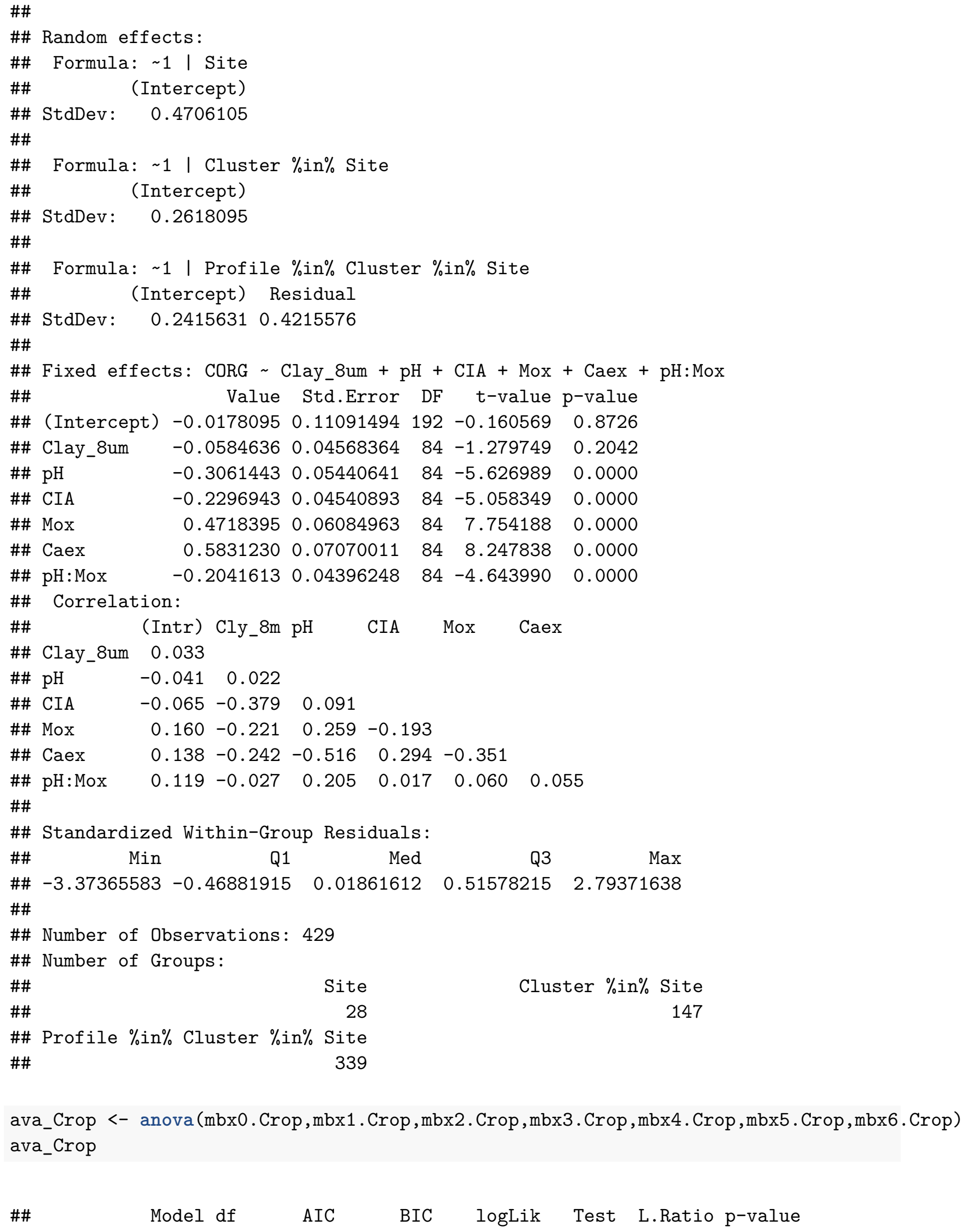




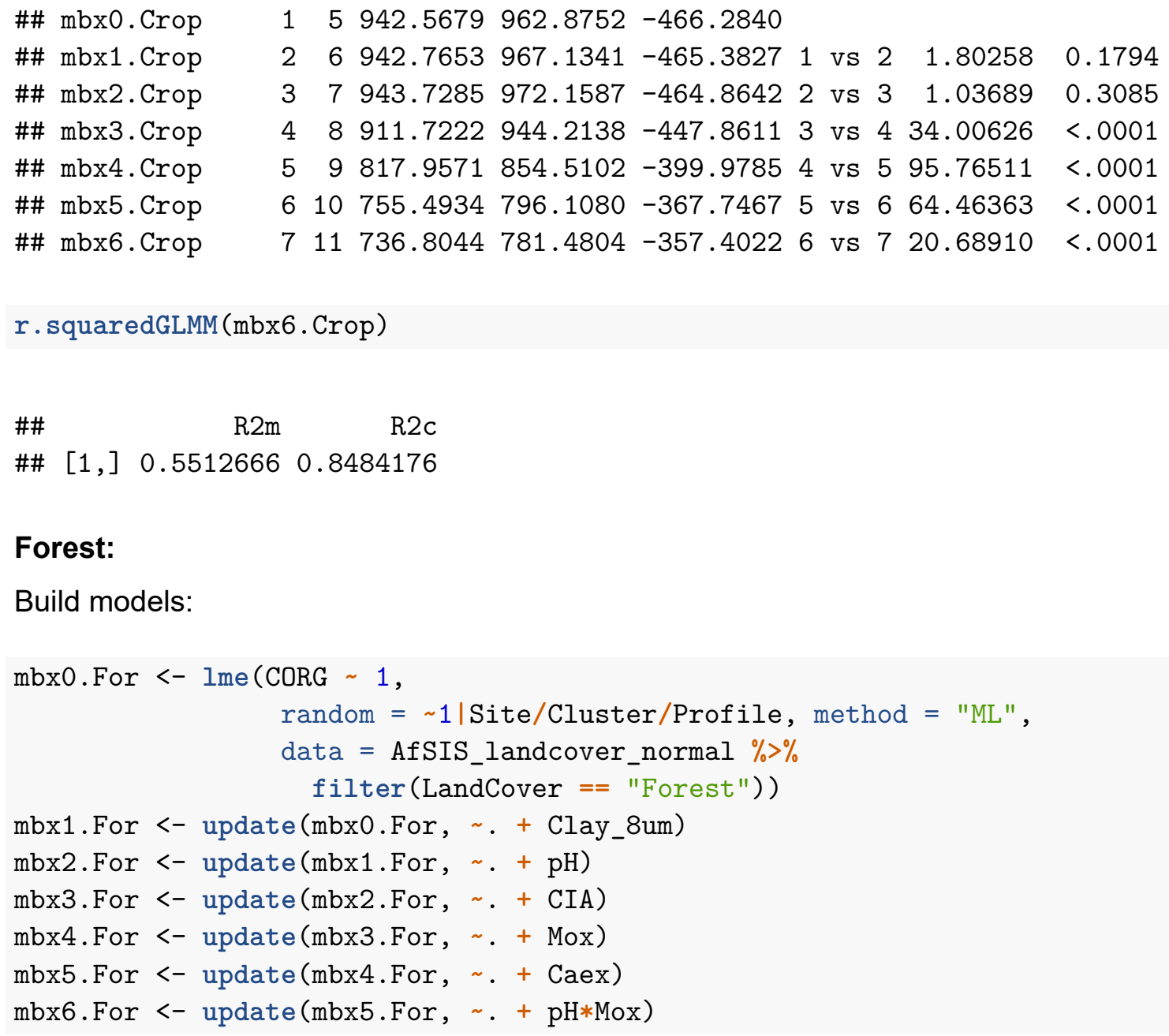

Forest:

Build models:

\section{Autocorrelation:}

vif_For <- as.data.frame(vif (mbx6.For))

vif_For_test <- $\max ($ vif_For $\$ " v i f(\operatorname{mbx} 6$. For $) ")<3.0$

VIF < 3.0: TRUE -> no autocorrelation

Diagnostic plots:

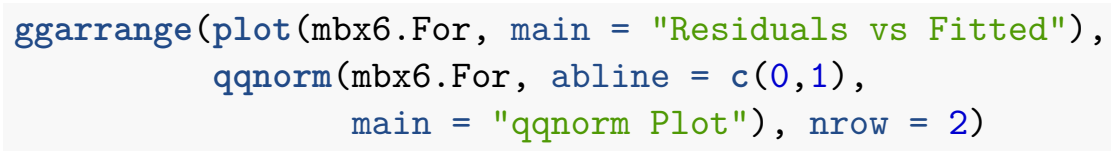




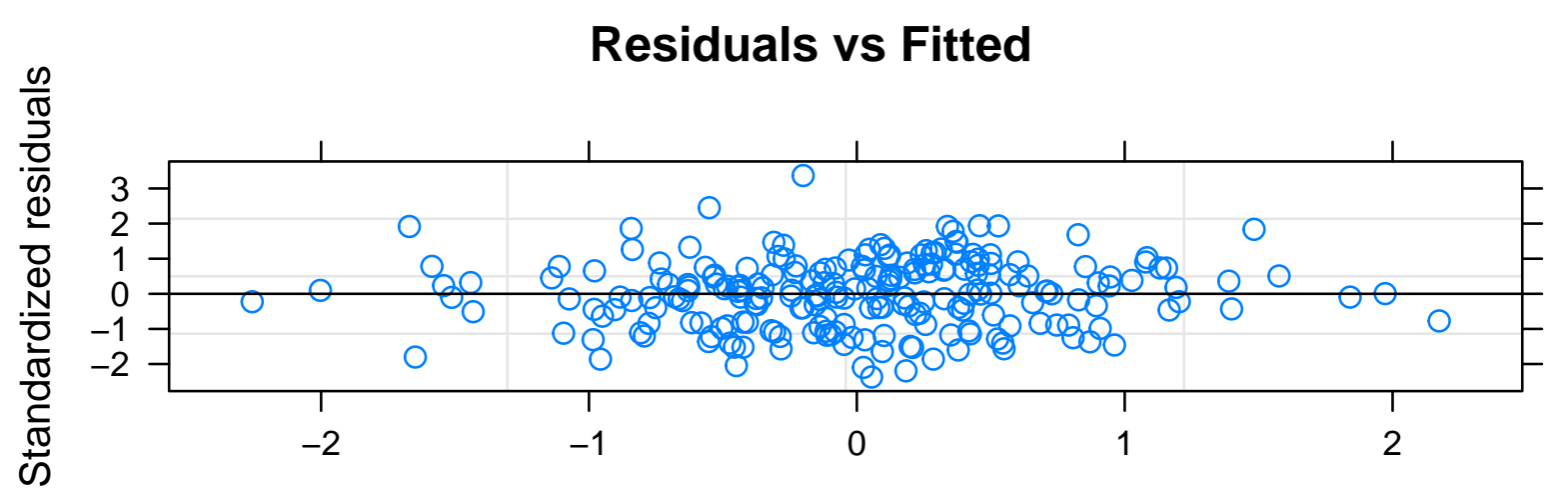

Fitted values

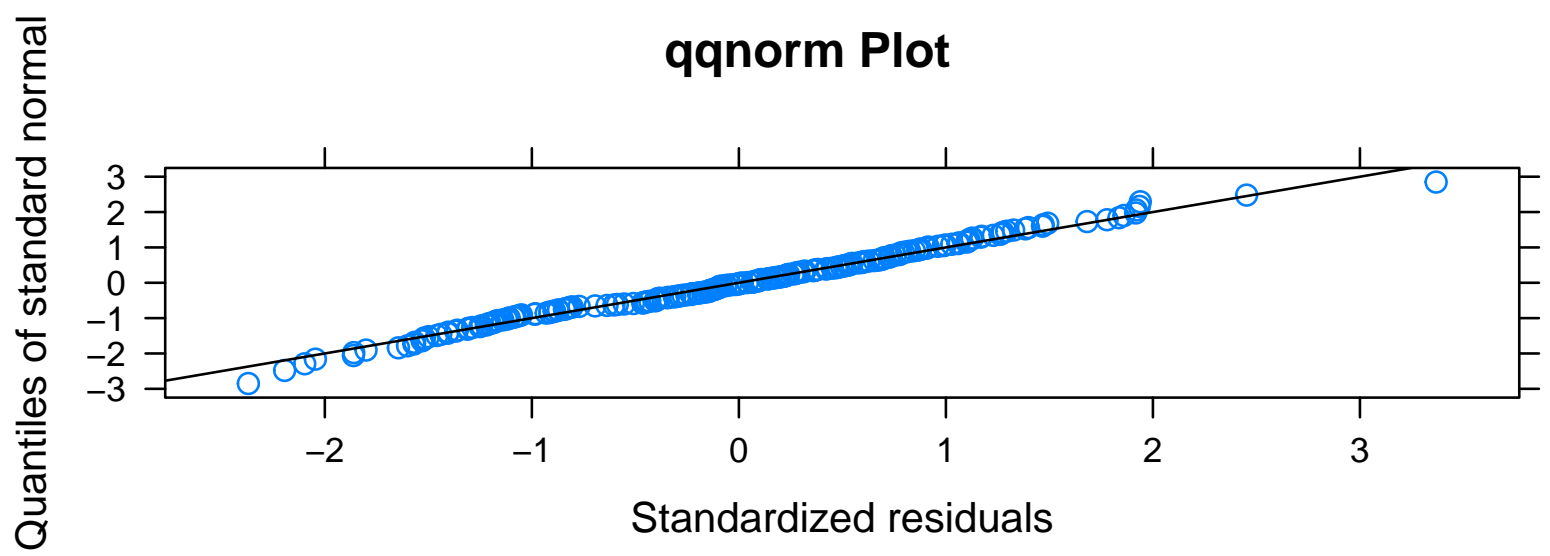

Model assumptions are met.

Anova output for all forest models (step-wise):

summary (mbx6. For)

\#\# Linear mixed-effects model fit by maximum likelihood

\#\# Data: AfSIS_landcover_normal \% $\%$ filter (LandCover == "Forest")

\#\# AIC BIC logLik

$\begin{array}{llll}\text { \#\# } \quad 532.3299 & 570.0527 & -255.1649\end{array}$

\#\#

\#\# Random effects:

\#\# Formula: $\sim 1$ | Site

\#\# (Intercept)

\#\# StdDev: 0.4733846

\#\#

\#\# Formula: $\sim 1$ | Cluster \%in\% Site

\#\# (Intercept)

\#\# StdDev: 0.2334054

\#\#

\#\# Formula: 1 | Profile \%in\% Cluster \%in\% Site

\#\# (Intercept) Residual

\#\# StdDev: $5.099371 \mathrm{e}-050.698566$ 


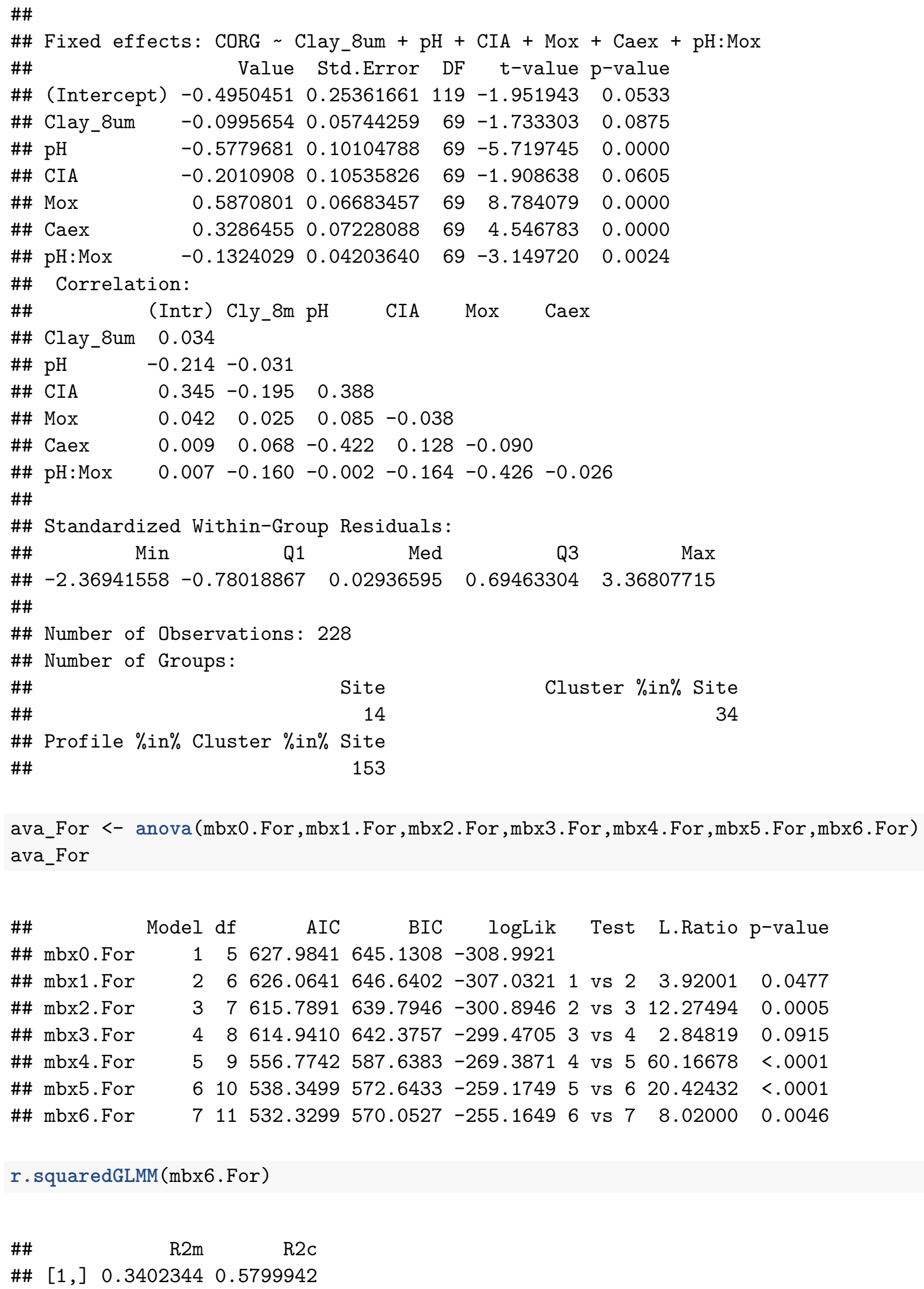




\section{Grassland:}

Build models:

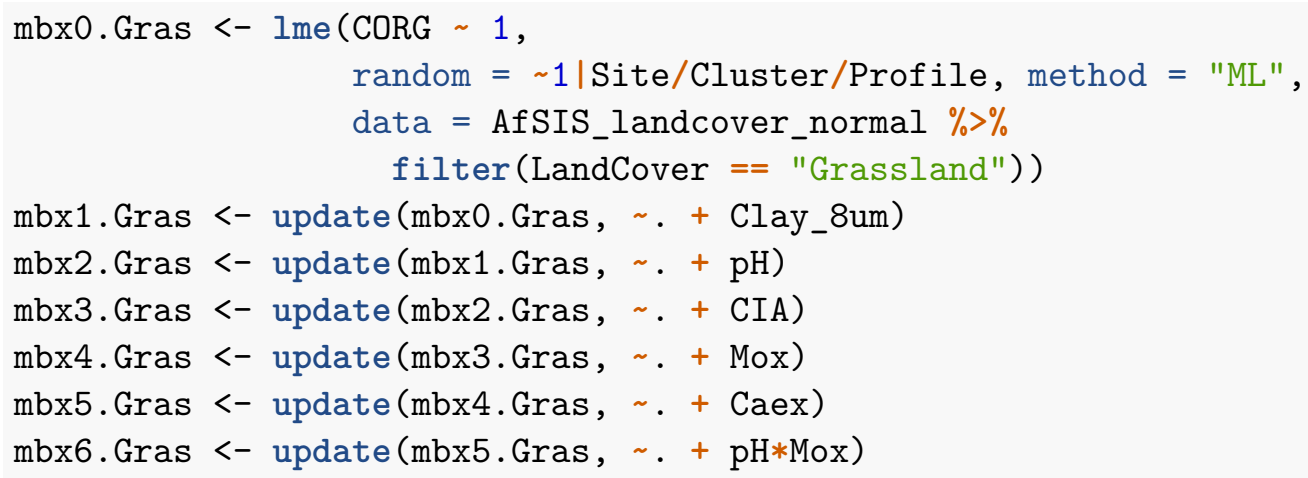

Autocorrelation:

vif_Gras <- as.data.frame (vif (mbx6.Gras))

vif_Gras_test <- max(vif_Gras\$"vif(mbx6.Gras)") < 3.0

VIF < 3.0: TRUE -> no autocorrelation

Diagnostic plots:

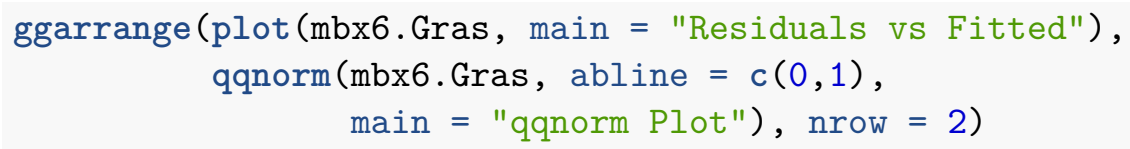




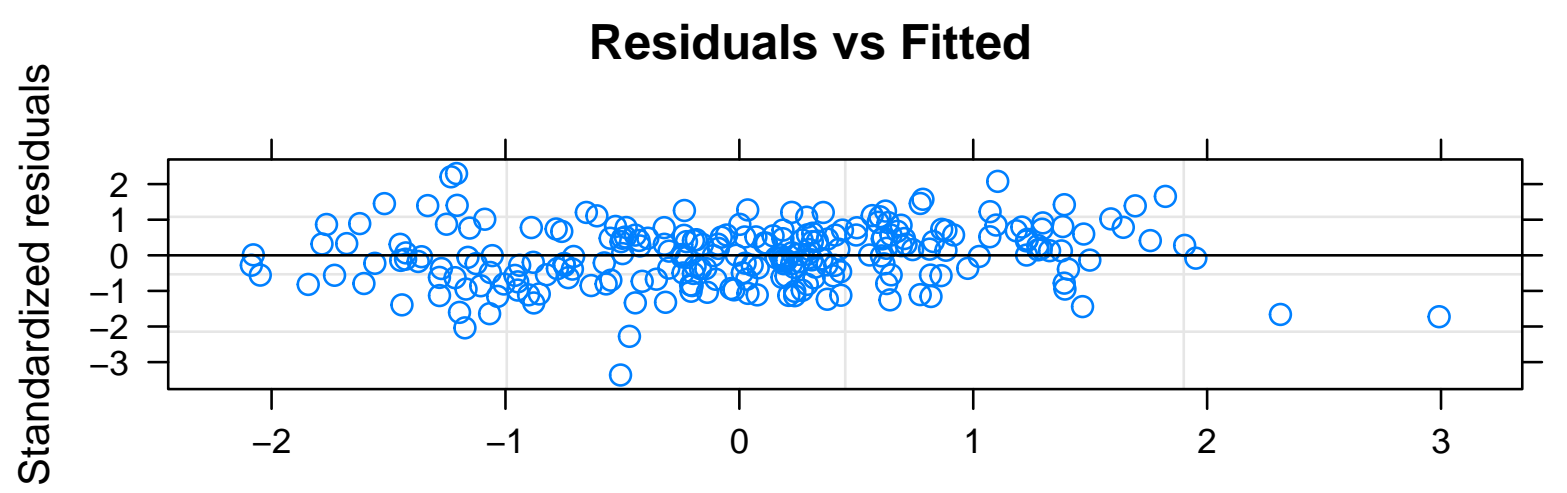

Fitted values

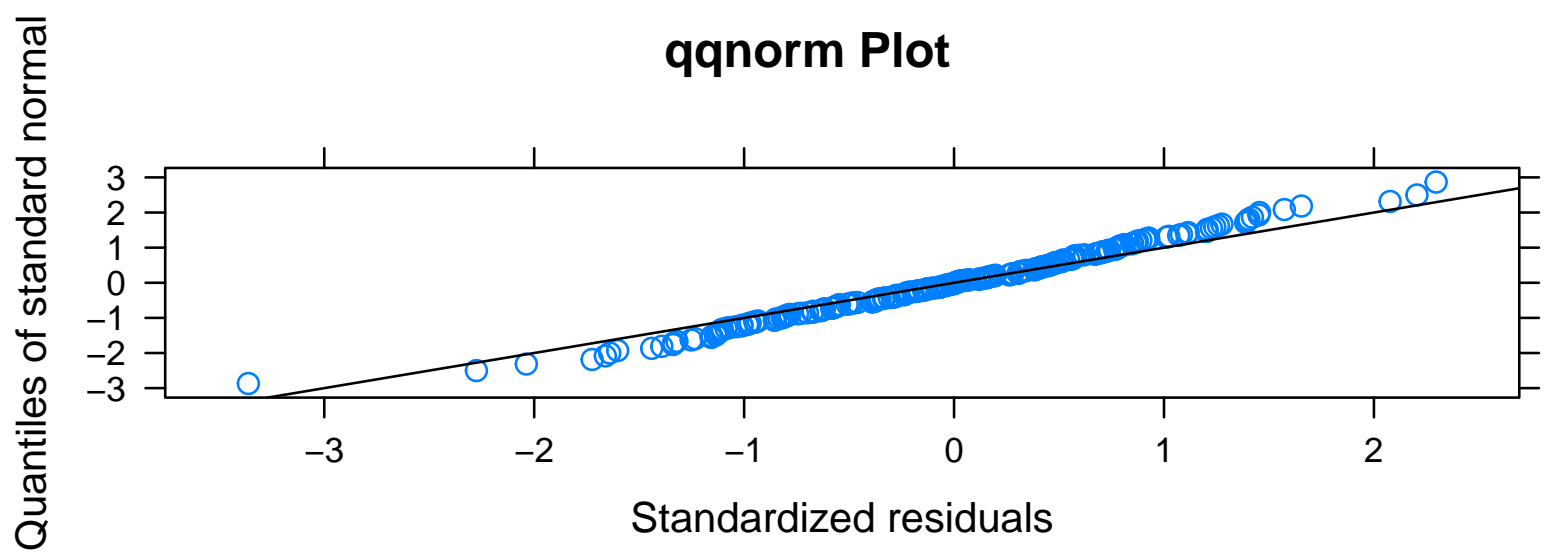

Model assumptions are met.

Anova output for all grassland models (step-wise):

summary (mbx6.Gras)

\#\# Linear mixed-effects model fit by maximum likelihood

\#\# Data: AfSIS_landcover_normal \% $\%$ filter(LandCover == "Grassland")

\#\# AIC BIC logLik

\#\# $\quad 352.6606 \quad 391.0389-165.3303$

\#\#

\#\# Random effects:

\#\# Formula: 1 | Site

\#\# (Intercept)

\#\# StdDev: 0.4777654

\#\#

\#\# Formula: 1 | Cluster \%in\% Site

\#\# (Intercept)

\#\# StdDev: 0.3017503

\#\#

\#\# Formula: 1 | Profile \%in\% Cluster \%in $\%$ Site

\#\# (Intercept) Residual

\#\# StdDev: 2.171951e-05 0.3467708 


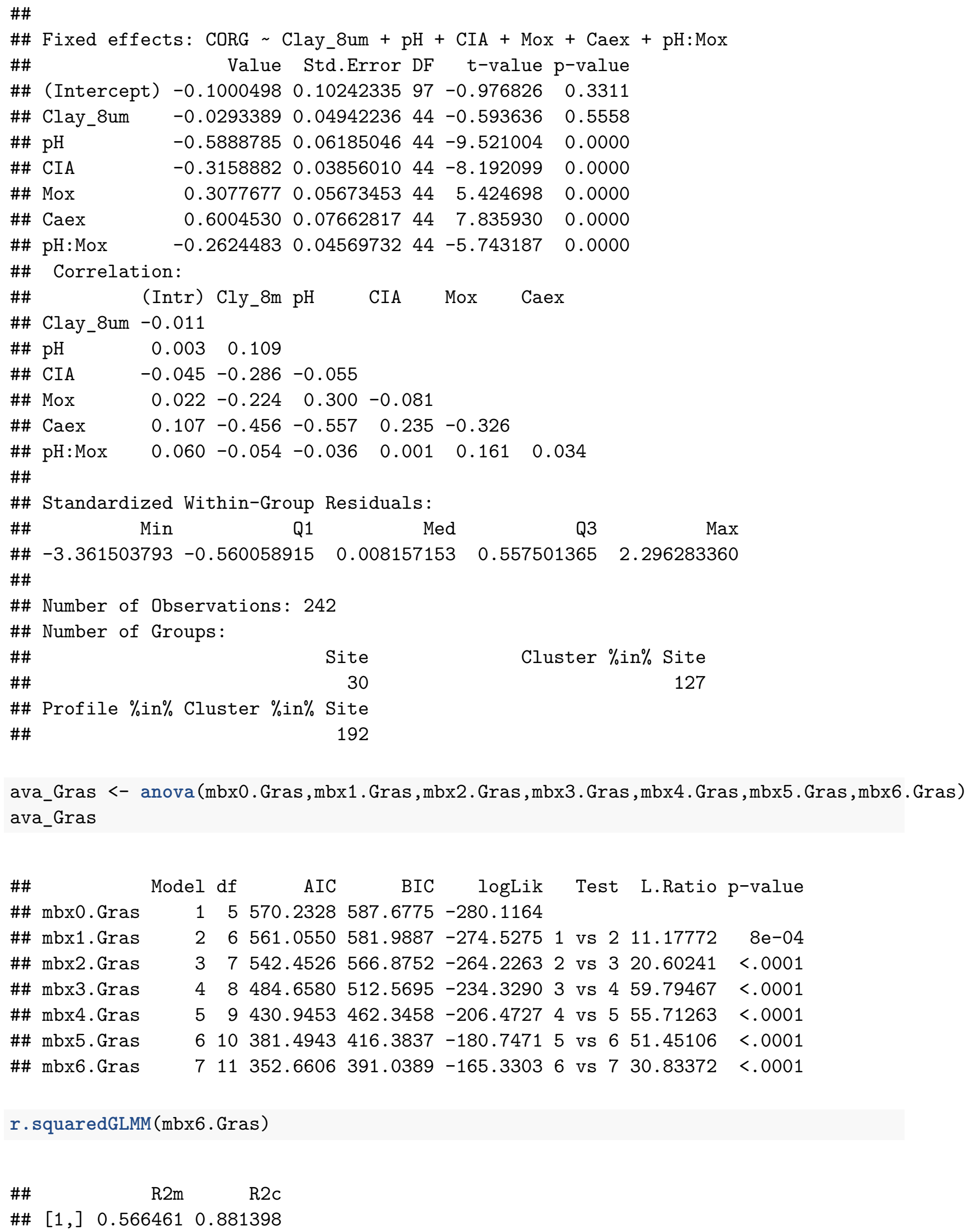




\section{Other:}

Build models:

$\operatorname{mbx} 0.0$ th $<-1 \mathrm{me}(\mathrm{CORG} \sim 1$,

random $=\sim 1 \mid$ Site $/$ Cluster $/$ Profile, method = "ML",

data $=$ AfSIS_landcover_normal $\%>\%$

filter (LandCover $==$ "Other"))

mbx1.0th <- update (mbx0.0th, . + Clay_8um)

mbx2.0th <- update (mbx1.0th, . + pH)

mbx3.0th <- update $(\operatorname{mbx} 2.0 t h, \sim .+$ CIA $)$

mbx4.0th <- update (mbx3.0th, . + Mox)

mbx5.0th <- update $(\operatorname{mbx} 4.0$ th, $\sim+$ Caex $)$

mbx6.0th <- update(mbx5.0th, . + pH*Mox)

\section{Autocorrelation:}

vif_Oth <- as.data.frame(vif (mbx6.0th))

vif_Oth_test <- $\max ($ vif_Oth\$"vif(mbx6.0th)" $)<3.0$

VIF < 3.0: TRUE -> no autocorrelation

Diagnostic plots:

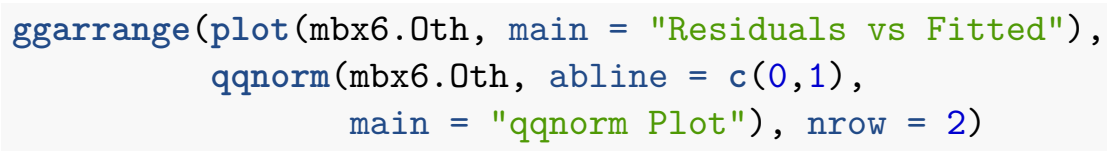




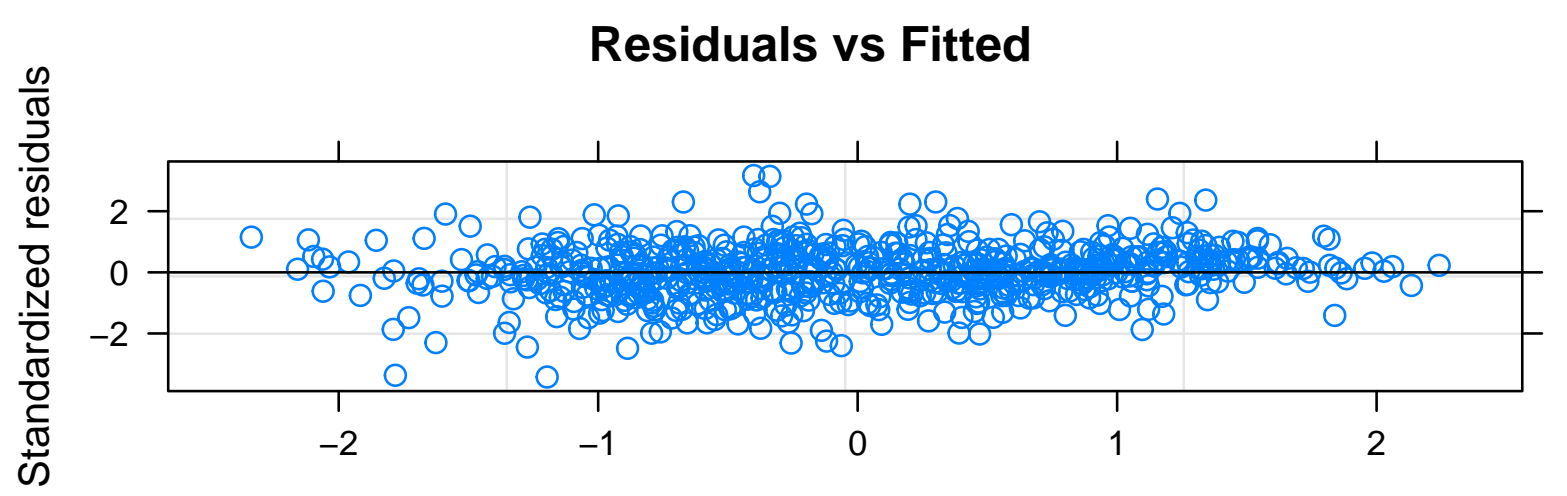

Fitted values

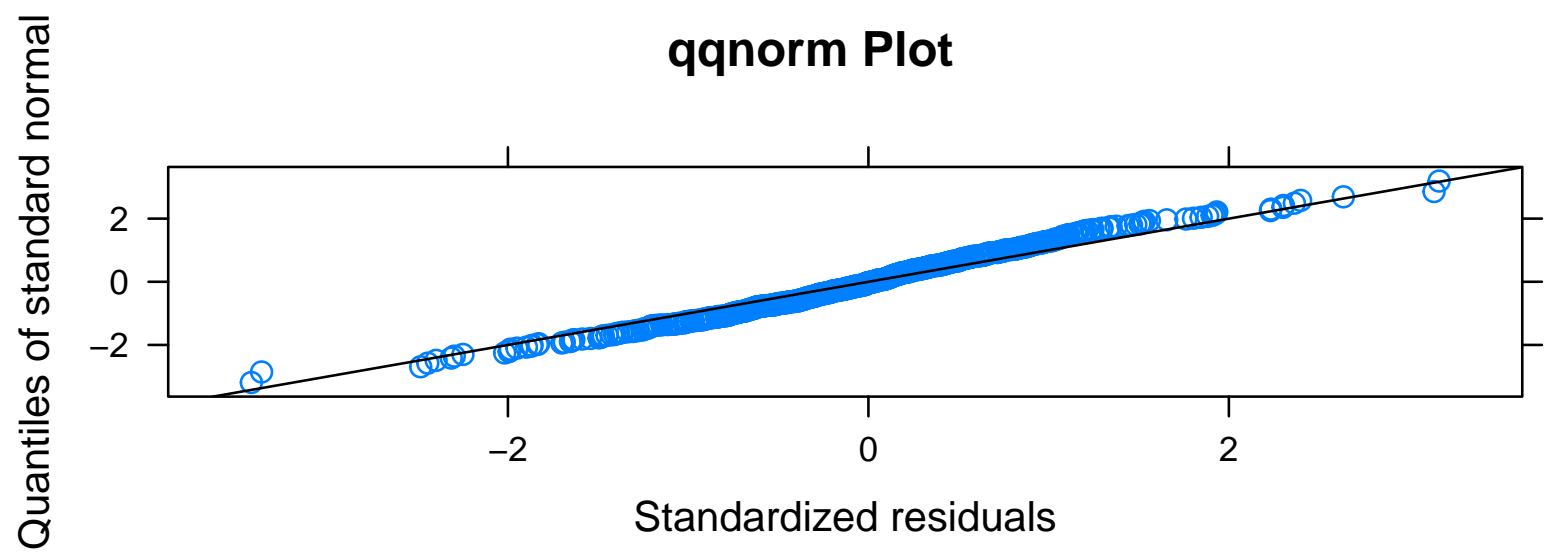

Model assumptions are met.

Anova output for all other models (step-wise):

summary (mbx6.0th)

\#\# Linear mixed-effects model fit by maximum likelihood

\#\# Data: AfSIS_landcover_normal \% \% filter (LandCover == "Other")

\#\# AIC BIC logLik

\#\# $\quad 1011.661 \quad 1061.754 \quad-494.8305$

\#\#

\#\# Random effects:

\#\# Formula: $\sim 1$ | Site

\#\# (Intercept)

\#\# StdDev: 0.5366225

\#\#

\#\# Formula: $\sim 1$ | Cluster \%in\% Site

\#\# (Intercept)

\#\# StdDev: 0.2049804

\#\#

\#\# Formula: $~ 1$ | Profile \%in\% Cluster \%in\% Site

\#\# (Intercept) Residual

\#\# StdDev: 0.19051830 .3808144 


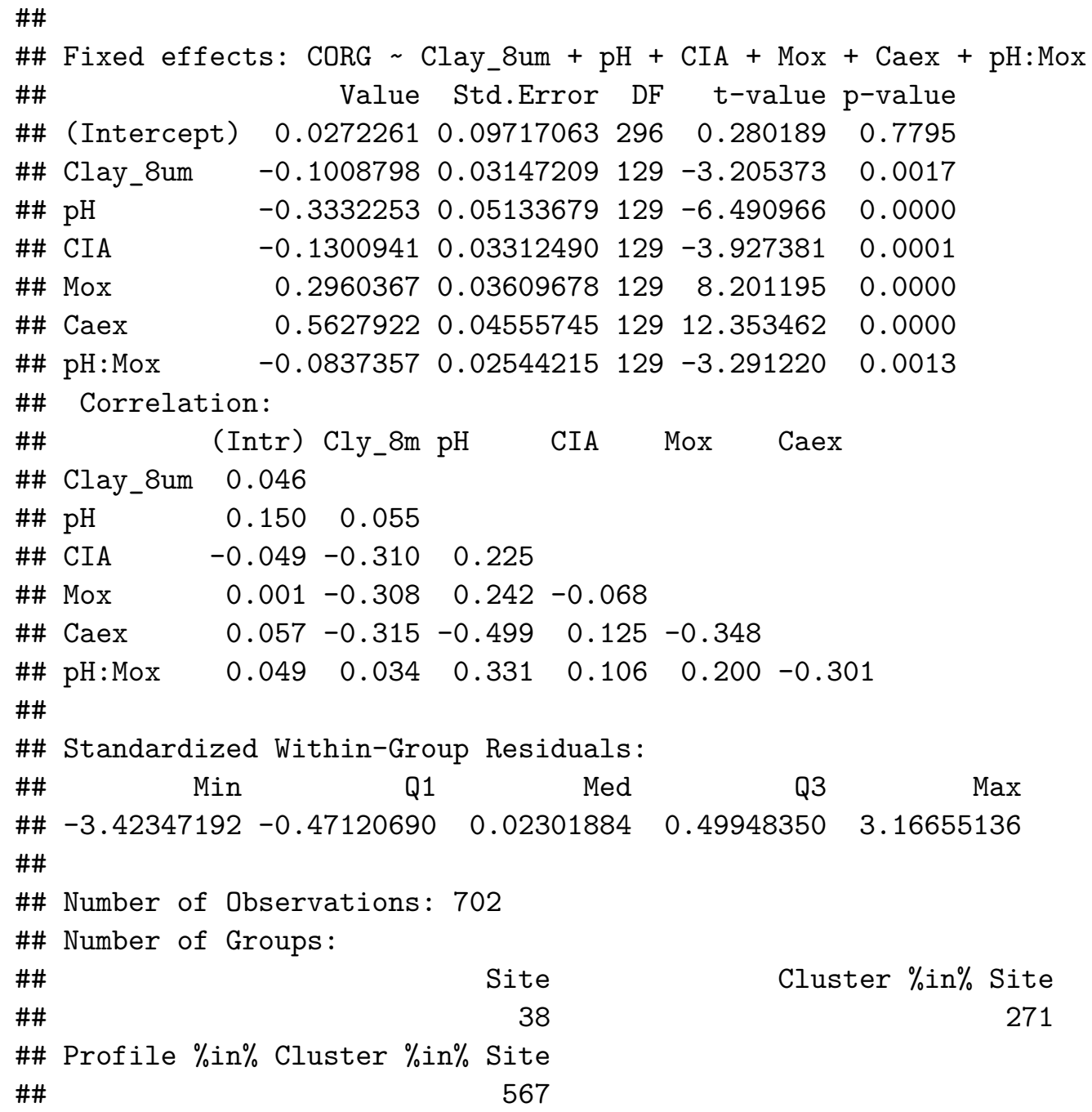




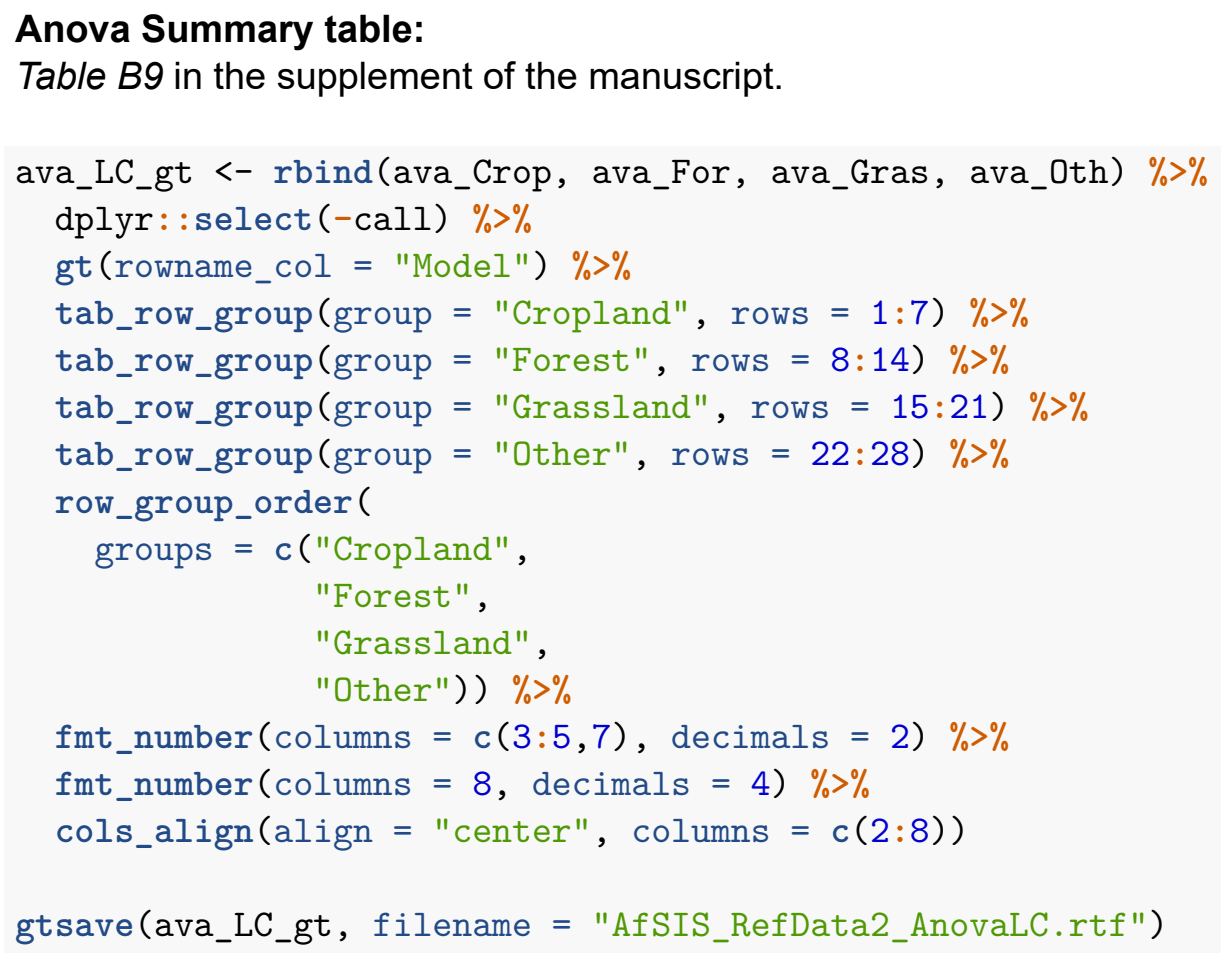

\subsubsection{Plotting}

This section contains the code to reproduce Figure 4.

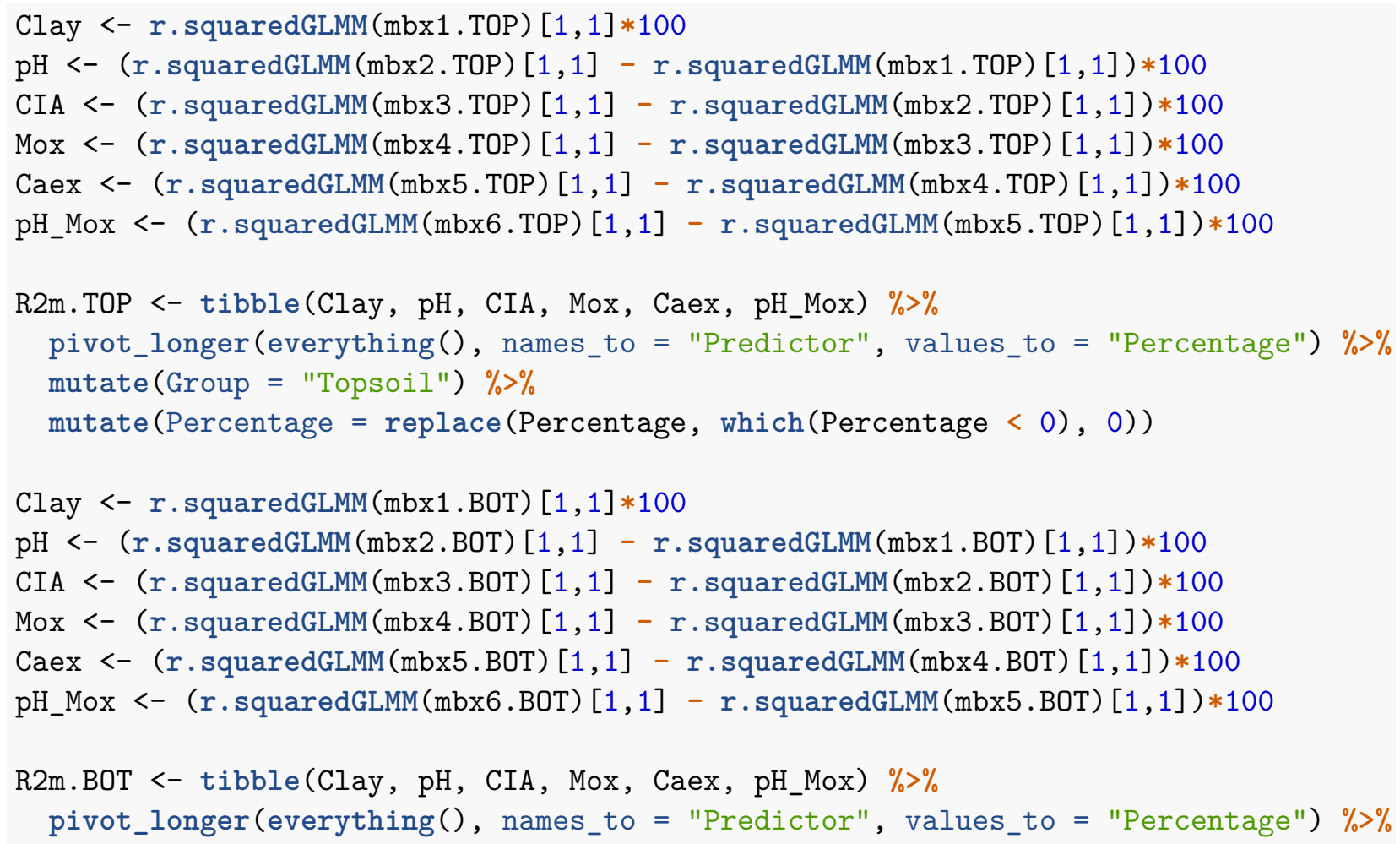




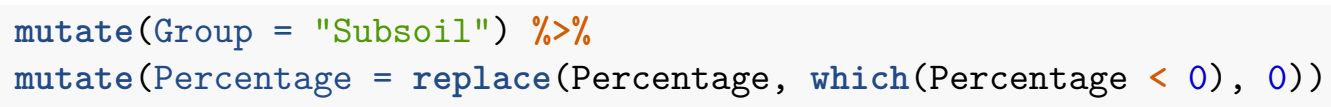


R2m.alk <- tibble(Clay, pH, CIA, Mox, Caex, pH_Mox) \% \%

pivot_longer(everything (), names_to = "Predictor", values_to = "Percentage") $\%>$ mutate (Group = "alkaline") $\%>\%$

mutate $($ Percentage $=\operatorname{replace}($ Percentage, which $($ Percentage $<0), 0))$

R2m.pH <- rbind(R2m.sacid, R2m.macid, R2m.neut, R2m.alk)

$\mathrm{R} 2 \mathrm{~m} \cdot \mathrm{pH} \$$ Group <- factor $(\mathrm{R} 2 \mathrm{~m} \cdot \mathrm{pH} \$$ Group,

$$
\begin{gathered}
\text { levels = c("strongly acidic", "moderately acidic", } \\
\text { "neutral", "alkaline")) }
\end{gathered}
$$

R2m.pH\$Predictor <- factor $(\mathrm{R} 2 \mathrm{~m} \cdot \mathrm{pH} \$$ Predictor,

$$
\begin{array}{r}
\text { levels = c("Depth", "LandCover", } \\
\text { "Clay", "pH", "CIA", "Mox", "Caex", "pH_Mox")) }
\end{array}
$$

\#\#\# CIA

CIA $<-0$

Clay <- r.squaredGLMM (mbx 1.CIAmod) $[1,1] * 100$

$\mathrm{pH}<-(\mathrm{r}$.squaredGLMM (mbx2.CIAmod) $[1,1]-\mathrm{r}$.squaredGLMM (mbx1.CIAmod) $[1,1]) * 100$

Mox <- (r.squaredGLMM (mbx3.CIAmod) $[1,1]-r$.squaredGLMM (mbx2.CIAmod) $[1,1]) * 100$

Caex <- (r.squaredGLMM (mbx4.CIAmod) $[1,1]$ - r.squaredGLMM (mbx3.CIAmod) $[1,1]) * 100$

pH_Mox <- (r.squaredGLMM (mbx5.CIAmod) $[1,1]$ - r.squaredGLMM (mbx4.CIAmod) $[1,1]) * 100$

R2m.CIAmod <- tibble(Clay, pH, CIA, Mox, Caex, pH_Mox) $\%>\%$

pivot_longer(everything(), names_to = "Predictor", values_to = "Percentage") $\%>$

mutate (Group = "moderate") $\%>\%$

mutate $($ Percentage $=$ replace $($ Percentage, which $($ Percentage $<0), 0))$

Clay <- r.squaredGLMM (mbx1. CIAhigh) $[1,1] * 100$

$\mathrm{pH}<-$ ( $r$.squaredGLMM (mbx2.CIAhigh) $[1,1]$ - r.squaredGLMM (mbx1.CIAhigh) $[1,1]) * 100$

Mox <- (r.squaredGLMM (mbx3.CIAhigh) $[1,1]$ - r.squaredGLMM (mbx2.CIAhigh) $[1,1]) * 100$

Caex <- (r.squaredGLMM (mbx4.CIAhigh) $[1,1]$ - r.squaredGLMM (mbx3.CIAhigh) $[1,1]) * 100$

pH_Mox <- (r.squaredGLMM (mbx5.CIAhigh) $[1,1]$ - r.squaredGLMM (mbx4.CIAhigh) $[1,1]) * 100$

R2m.CIAhigh <- tibble(Clay, pH, CIA, Mox, Caex, pH_Mox) $\%>\%$

pivot_longer(everything (), names_to = "Predictor", values_to $=$ "Percentage") $\%>\%$

mutate (Group = "high") $\%>\%$

mutate $($ Percentage $=\operatorname{replace}($ Percentage, which $($ Percentage $<0), 0))$

R2m.CIA <- rbind(R2m.CIAmod, R2m.CIAhigh)

R2m.CIA\$Group <- factor (R2m.CIA\$Group,

levels = c("moderate", "high"))

R2m.CIA\$Predictor <- factor (R2m.CIA\$Predictor,

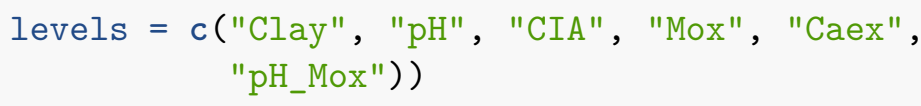

\#\#\# Number of wet month 
Clay <- r.squaredGLMM (mbx1.0wet) $[1,1] * 100$

$\mathrm{pH}<-(r$.squaredGLMM (mbx2.0wet) $[1,1]-r$.squaredGLMM (mbx1.0wet) $[1,1]) * 100$

CIA <- (r.squaredGLMM (mbx3.0wet) $[1,1]$ - r.squaredGLMM (mbx2.0wet) $[1,1]) * 100$

Mox <- (r.squaredGLMM $(\operatorname{mbx} 4.0$ wet $)[1,1]$ - r.squaredGLMM (mbx3.0wet) $[1,1]) * 100$

Caex <- (r.squaredGLMM (mbx5.0wet) $[1,1]-r$.squaredGLMM (mbx 4.0wet) $[1,1]) * 100$

pH_Mox <- $(r$.squaredGLMM $(\operatorname{mbx} 6$.0wet $)[1,1]-r$.squaredGLMM $(\operatorname{mbx} 5.0$ wet $)[1,1]) * 100$

R2m.0wt <- tibble(Clay, pH, CIA, Mox, Caex, pH_Mox) \%>\%

pivot_longer(everything(), names_to = "Predictor", values_to = "Percentage") $\%>\%$ mutate (Group = "0") $\%>\%$

mutate $($ Percentage $=$ replace $($ Percentage, which $($ Percentage $<0), 0))$

Clay <- r.squaredGLMM (mbx1.1_3wet) $[1,1] * 100$

$\mathrm{pH}<-\left(r\right.$.squaredGLMM (mbx2.1_3wet) $[1,1]$ - r.squaredGLMM $\left.\left(\operatorname{mbx} 1.1 \_3 w e t\right)[1,1]\right) * 100$

CIA <- (r.squaredGLMM (mbx3.1_3wet) $[1,1]$ - r.squaredGLMM (mbx2.1_3wet) $[1,1]) * 100$

Mox <- (r.squaredGLMM (mbx4.1_3wet) $[1,1]$ - r.squaredGLMM (mbx3.1_3wet) $[1,1]) * 100$

Caex <- (r.squaredGLMM (mbx5.1_3wet) $[1,1]$ - r.squaredGLMM (mbx4.1_3wet) $[1,1]) * 100$

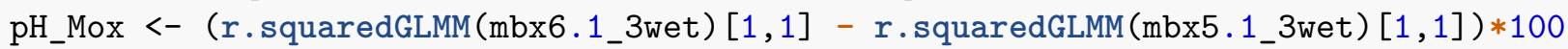

R2m.1_3wt <- tibble(Clay, pH, CIA, Mox, Caex, pH_Mox) $\%>\%$

pivot_longer(everything (), names_to = "Predictor", values_to = "Percentage") $\%>\%$ mutate (Group = "1-3") $\%>\%$

mutate $($ Percentage $=$ replace $($ Percentage, which $($ Percentage $<0), 0))$

Clay <- r.squaredGLMM (mbx1.4_7wet) $[1,1] * 100$

$\mathrm{pH}<-\left(r\right.$.squaredGLMM $\left(\mathrm{mbx} 2.4 \_7 \mathrm{wet}\right)[1,1]$ - r.squaredGLMM $\left.\left(\operatorname{mbx} 1.4 \_7 \mathrm{wet}\right)[1,1]\right) * 100$

CIA <- (r.squaredGLMM (mbx3.4_7wet) $[1,1]$ - r.squaredGLMM $\left(m b x 2.4 \_7\right.$ wet $\left.)[1,1]\right) * 100$

Mox <- (r.squaredGLMM (mbx4.4_7wet) $[1,1]$ - r.squaredGLMM (mbx3.4_7wet) $[1,1]) * 100$

Caex <- (r.squaredGLMM (mbx5.4_7wet) $[1,1]$ - r.squaredGLMM $\left.\left(\operatorname{mbx} 4.4 \_7 \mathrm{wet}\right)[1,1]\right) * 100$

pH_Mox <- (r.squaredGLMM $\left(\operatorname{mbx} 6.4 \_7\right.$ wet $)[1,1]$ - r.squaredGLMM $(\operatorname{mbx} 5.4$ _ 7 wet $\left.)[1,1]\right) * 100$

R2m.4_7wt <- tibble(Clay, pH, CIA, Mox, Caex, pH_Mox) $\%>\%$

pivot_longer(everything () , names_to = "Predictor", values_to = "Percentage") $\%>$ mutate (Group = "4-7") $\%>\%$

mutate $($ Percentage $=$ replace $($ Percentage, which $($ Percentage $<0), 0))$

R2m.nWet <- rbind (R2m.0wt, R2m.1_3wt, R2m.4_7wt)

R2m.nWet\$Group <- factor (R2m.nWet\$Group,

levels = c("0", "1-3", "4-7"))

R2m.nWet\$Predictor <- factor $(R 2 m . n W e t \$ P r e d i c t o r$,

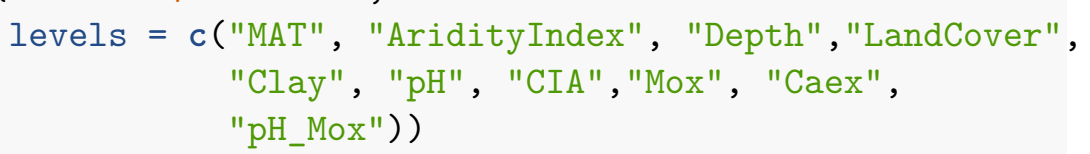

\#\#\# LandCover

Clay <- r.squaredGLMM (mbx1.Crop) $[1,1] * 100$ 
$\mathrm{pH}<-(r$.squaredGLMM (mbx2.Crop) $[1,1]-r$.squaredGLMM (mbx1.Crop) $[1,1]) * 100$ CIA <- (r.squaredGLMM (mbx3.Crop) $[1,1]$ - r.squaredGLMM (mbx2.Crop) $[1,1]) * 100$ Mox <- (r.squaredGLMM (mbx4.Crop) $[1,1]$ - r.squaredGLMM (mbx3.Crop) $[1,1]) * 100$ Caex <- (r.squaredGLMM (mbx5.Crop) $[1,1]$ - r.squaredGLMM (mbx4.Crop) $[1,1]) * 100$ pH_Mox <- (r.squaredGLMM (mbx6.Crop) $[1,1]$ - r.squaredGLMM (mbx5.Crop) $[1,1]) * 100$

R2m.Crop <- tibble(Clay, pH, CIA, Mox, Caex, pH_Mox) \%>\% pivot_longer(everything(), names_to = "Predictor", values_to = "Percentage") $\%>$ mutate (Group = "Cropland") $\%>\%$

mutate $($ Percentage $=$ replace $($ Percentage, which $($ Percentage $<0), 0))$

Clay <- r.squaredGLMM (mbx1.For) $[1,1] * 100$

$\mathrm{pH}<-$ ( $r$.squaredGLMM (mbx2.For) $[1,1]$ - r.squaredGLMM (mbx1.For) $[1,1]) * 100$

CIA <- (r.squaredGLMM (mbx3.For) $[1,1]$ - r.squaredGLMM (mbx2.For) $[1,1]) * 100$

Mox <- (r.squaredGLMM (mbx4.For) $[1,1]$ - r.squaredGLMM (mbx3.For) $[1,1]) * 100$

Caex <- (r.squaredGLMM (mbx5.For) $[1,1]$ - r.squaredGLMM (mbx4.For) $[1,1]) * 100$

pH_Mox <- (r.squaredGLMM (mbx6.For) $[1,1]$ - r.squaredGLMM (mbx5.For) $[1,1]) * 100$

R2m.For <- tibble(Clay, pH, CIA, Mox, Caex, pH_Mox) $\%>\%$

pivot_longer(everything(), names_to = "Predictor", values_to = "Percentage") $\%>\%$ mutate (Group = "Forest") $\%>\%$

mutate $($ Percentage $=$ replace $($ Percentage, which $($ Percentage $<0), 0))$

Clay <- r.squaredGLMM (mbx1.Gras) $[1,1] * 100$

$\mathrm{pH}<-(r . s q u a r e d G L M M(\operatorname{mbx} 2$.Gras) $[1,1]$ - r.squaredGLMM (mbx1.Gras) $[1,1]) * 100$

CIA <- (r.squaredGLMM (mbx3.Gras) $[1,1]$ - r.squaredGLMM (mbx2.Gras) $[1,1]) * 100$

Mox <- (r.squaredGLMM (mbx4.Gras) [1,1] - r.squaredGLMM (mbx3.Gras) $[1,1]) * 100$

Caex <- (r.squaredGLMM (mbx5.Gras) [1,1] - r.squaredGLMM (mbx4.Gras) $[1,1]) * 100$

pH_Mox <- (r.squaredGLMM (mbx6.Gras) $[1,1]$ - r.squaredGLMM (mbx5.Gras) $[1,1]) * 100$

R2m.Gras <- tibble(Clay, pH, CIA, Mox, Caex, pH_Mox) $\%>\%$ pivot_longer(everything (), names_to = "Predictor", values_to = "Percentage") $\%>\%$ mutate (Group = "Grassland") $\%>\%$

mutate $($ Percentage $=$ replace $($ Percentage, which $($ Percentage $<0), 0))$

Clay <- r.squaredGLMM (mbx1.0th) $[1,1] * 100$

$\mathrm{pH}<-(\mathrm{r}$. squaredGLMM $(\operatorname{mbx} 2.0 \mathrm{th})[1,1]-\mathrm{r}$.squaredGLMM $(\operatorname{mbx} 1.0 \mathrm{th})[1,1]) * 100$

CIA <- (r.squaredGLMM (mbx3.0th) $[1,1]$ - r.squaredGLMM $(\operatorname{mbx} 2.0$ th $)[1,1]) * 100$

Mox <- (r.squaredGLMM (mbx4.0th) [1,1] - r.squaredGLMM (mbx3.0th) $[1,1]) * 100$

Caex <- (r.squaredGLMM (mbx5.0th) $[1,1]$ - r.squaredGLMM $(\operatorname{mbx} 4.0$ th $)[1,1]) * 100$

pH_Mox <- $(r . s q u a r e d G L M M(m b x 6.0 t h)[1,1]$ - r.squaredGLMM $(\operatorname{mbx} 5.0 t h)[1,1]) * 100$

R2m.Oth <- tibble(Clay, pH, CIA, Mox, Caex, pH_Mox) $\%>\%$

pivot_longer(everything () , names_to = "Predictor", values_to = "Percentage") $\%>\%$ mutate (Group = "Other") $\%>\%$

mutate $($ Percentage $=$ replace $($ Percentage, which $($ Percentage $<0), 0))$ 
R2m.LC <- rbind(R2m.Crop, R2m.For, R2m.Gras, R2m.Oth)

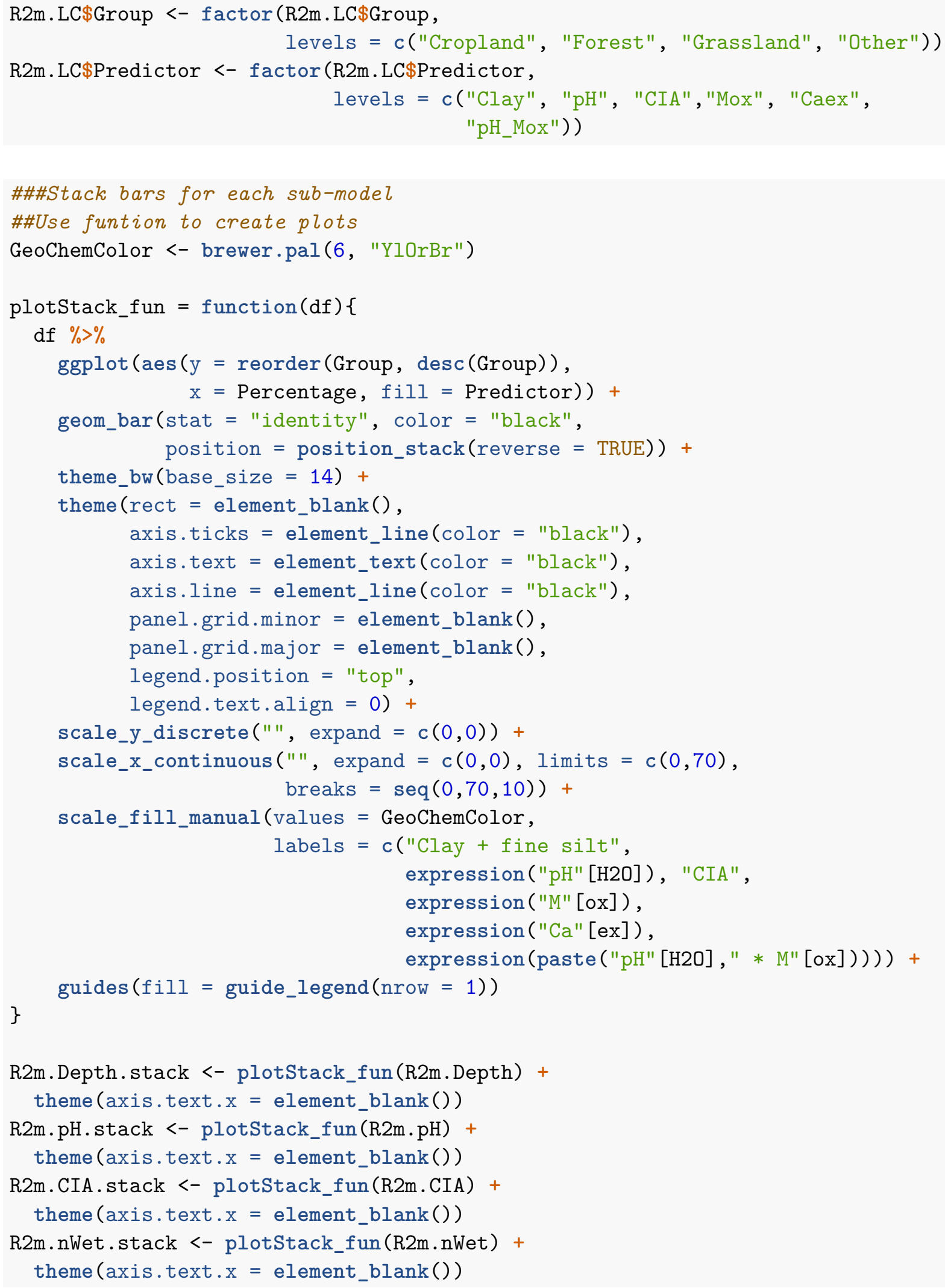




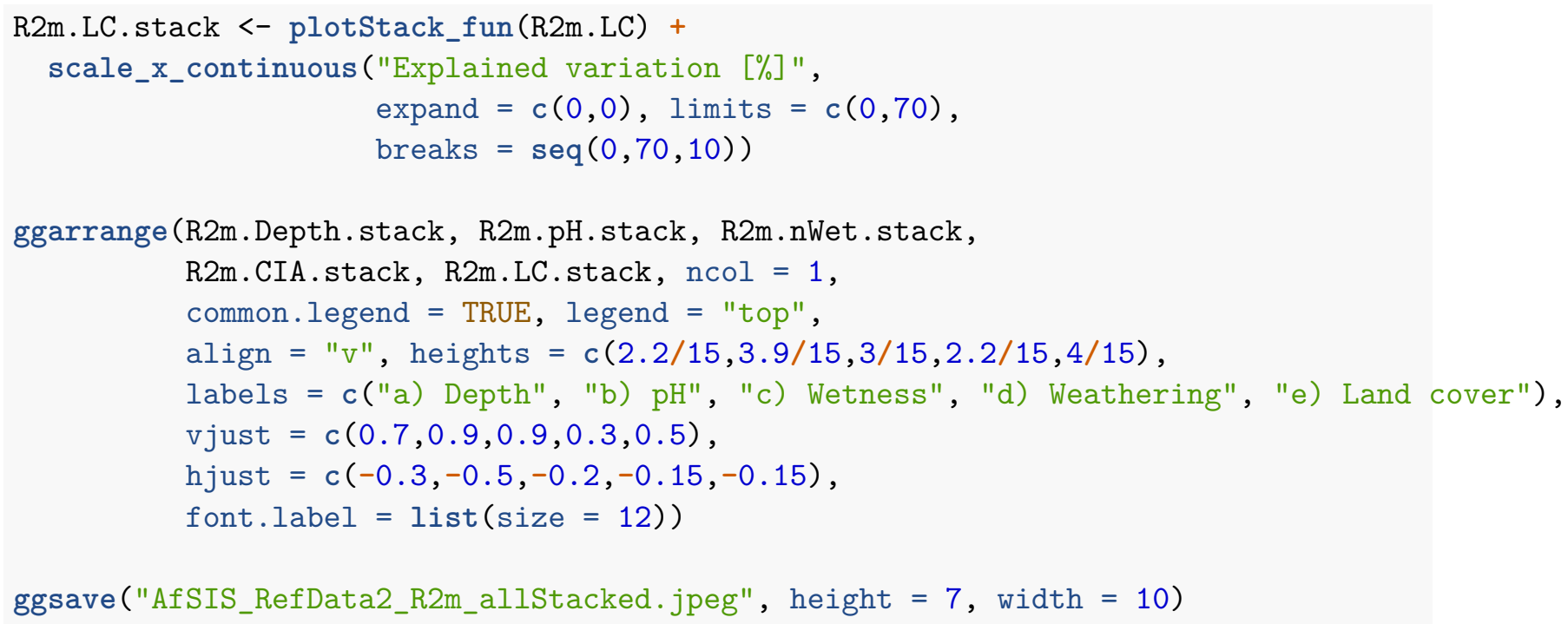

\subsubsection{Summary table}

This section contains the code to reproduce Table B2.

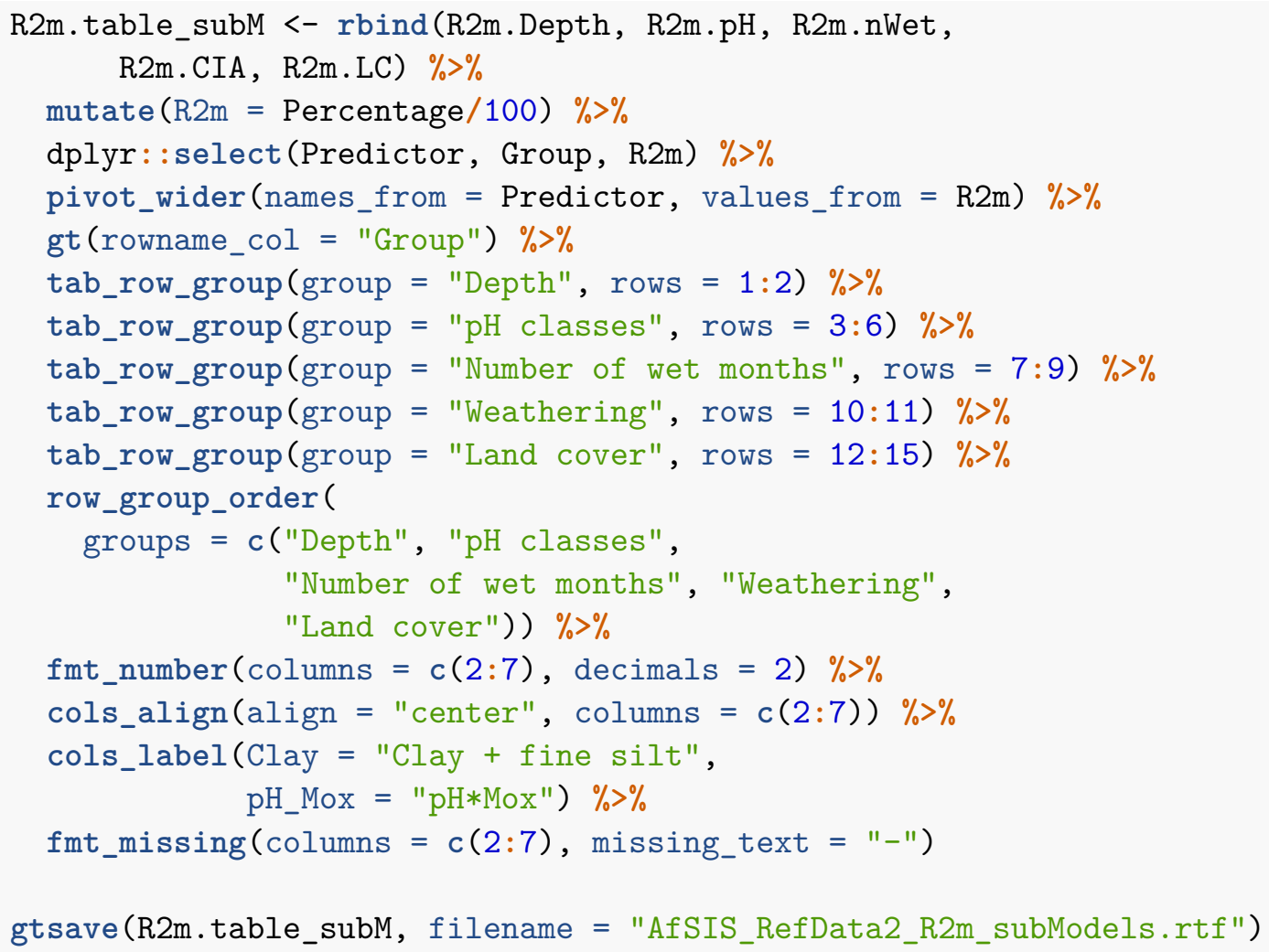




\section{Regression tree analysis}

This chapter contains all the code for the regression tree analyses, including the pruning, the spatial cross-validation and plotting of the trees. The modelling has been done for both depth intervals seperately.

\subsection{Modelling}

Two seperate trees will be build (topsoil vs subsoil). Otherwise, depth will be used most of the time to build the trees.

\section{Topsoil:}

Filter the dataset:

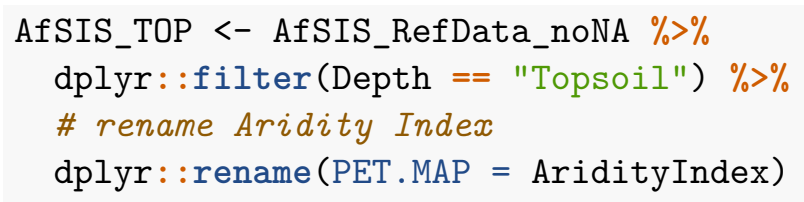

Build regression tree:

RT_TOP <- rpart: $: \operatorname{rpart}($ formula $=$ CORG $\sim$ MAT + PET.MAP + pH + Alox + CIA + Caex +
Feox + Clay_8um + LandCover, data $=$ AfSIS_TOP, method $=$ "anova" $)$

Prune model with grid search:

Code source: https://uc-r.github.io/regression_trees

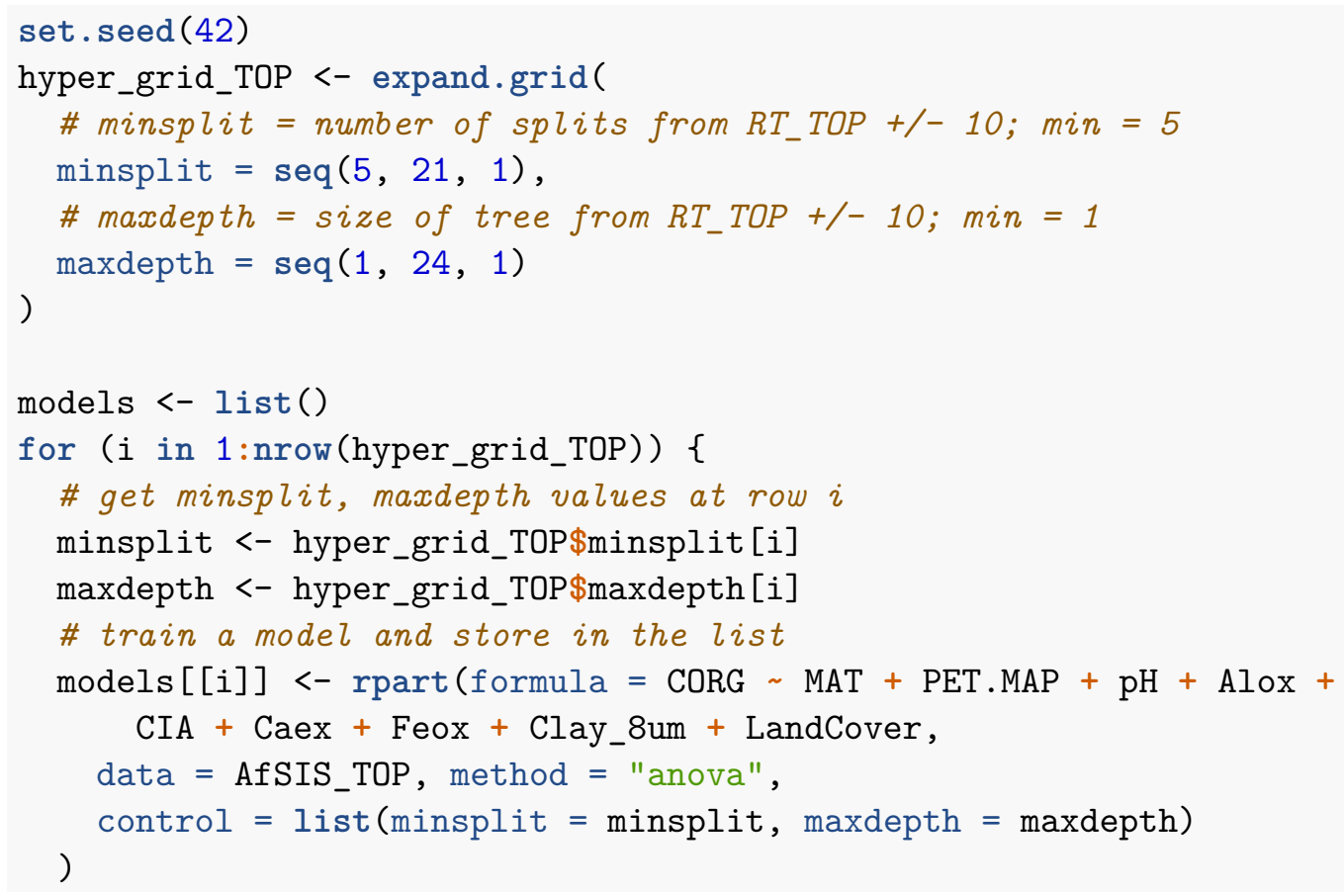




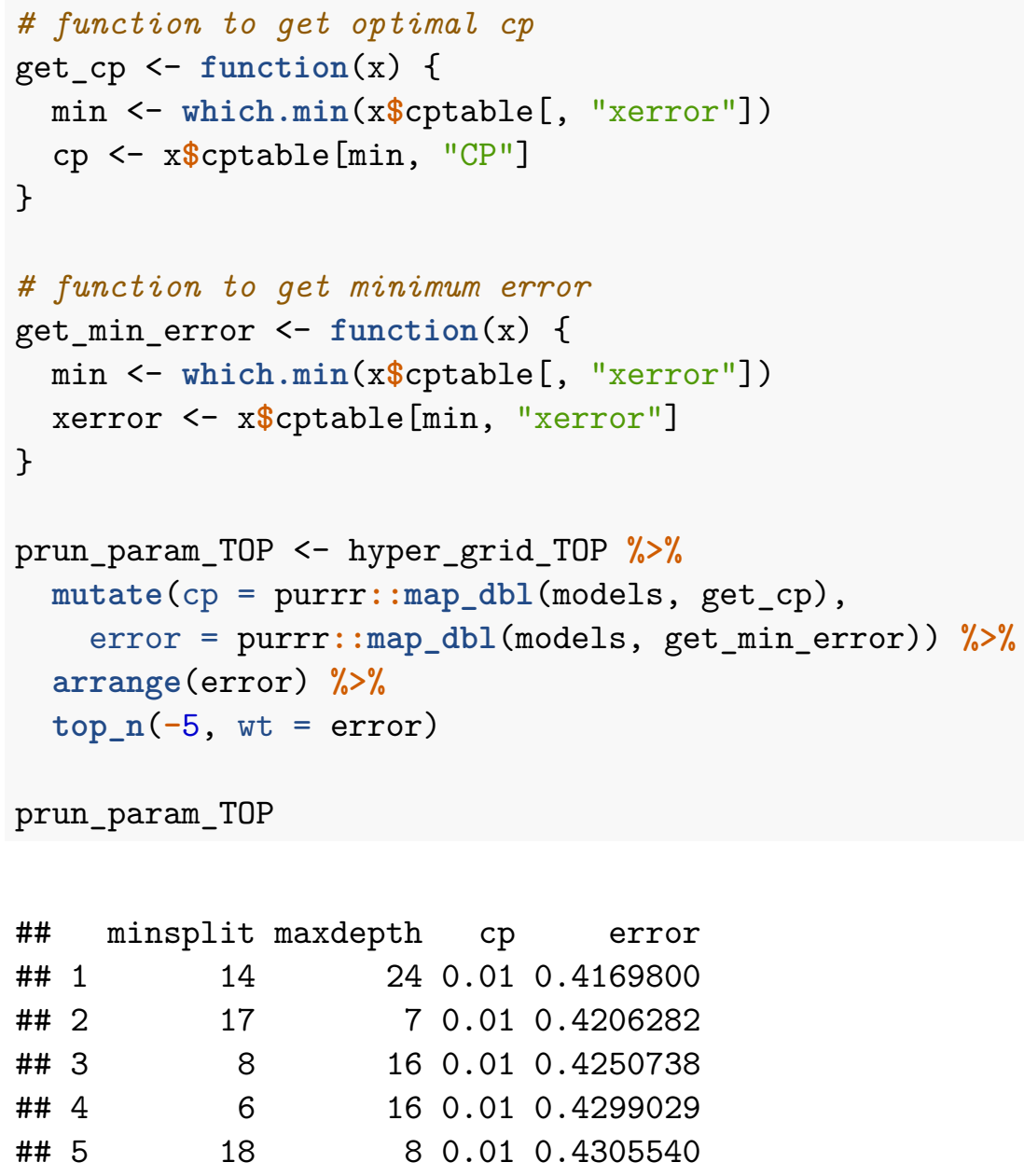

\section{Pruned regression tree:}

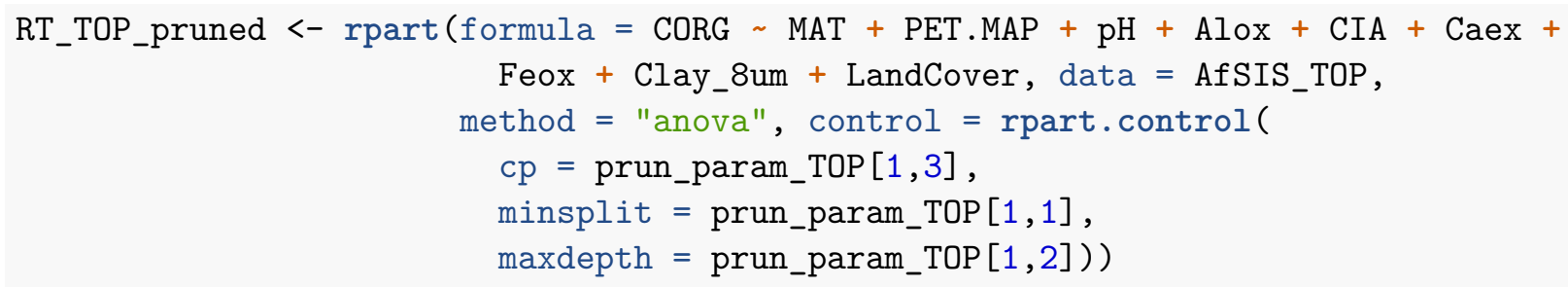

\section{Subsoil:}

\section{Filter dataset:}

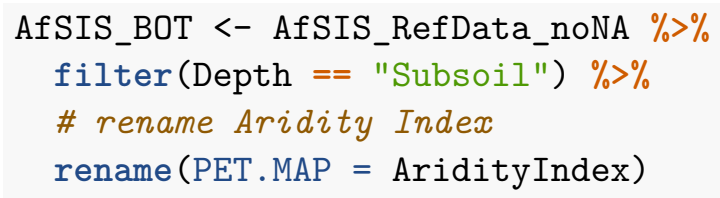




\section{Build regression tree:}

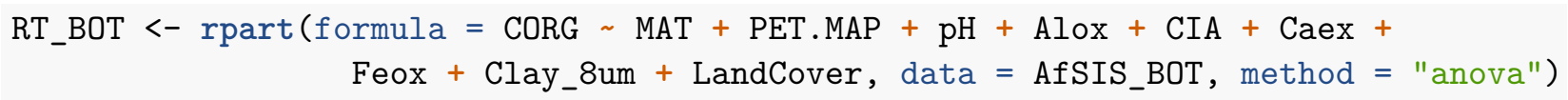

\section{Prune model with grid search:}

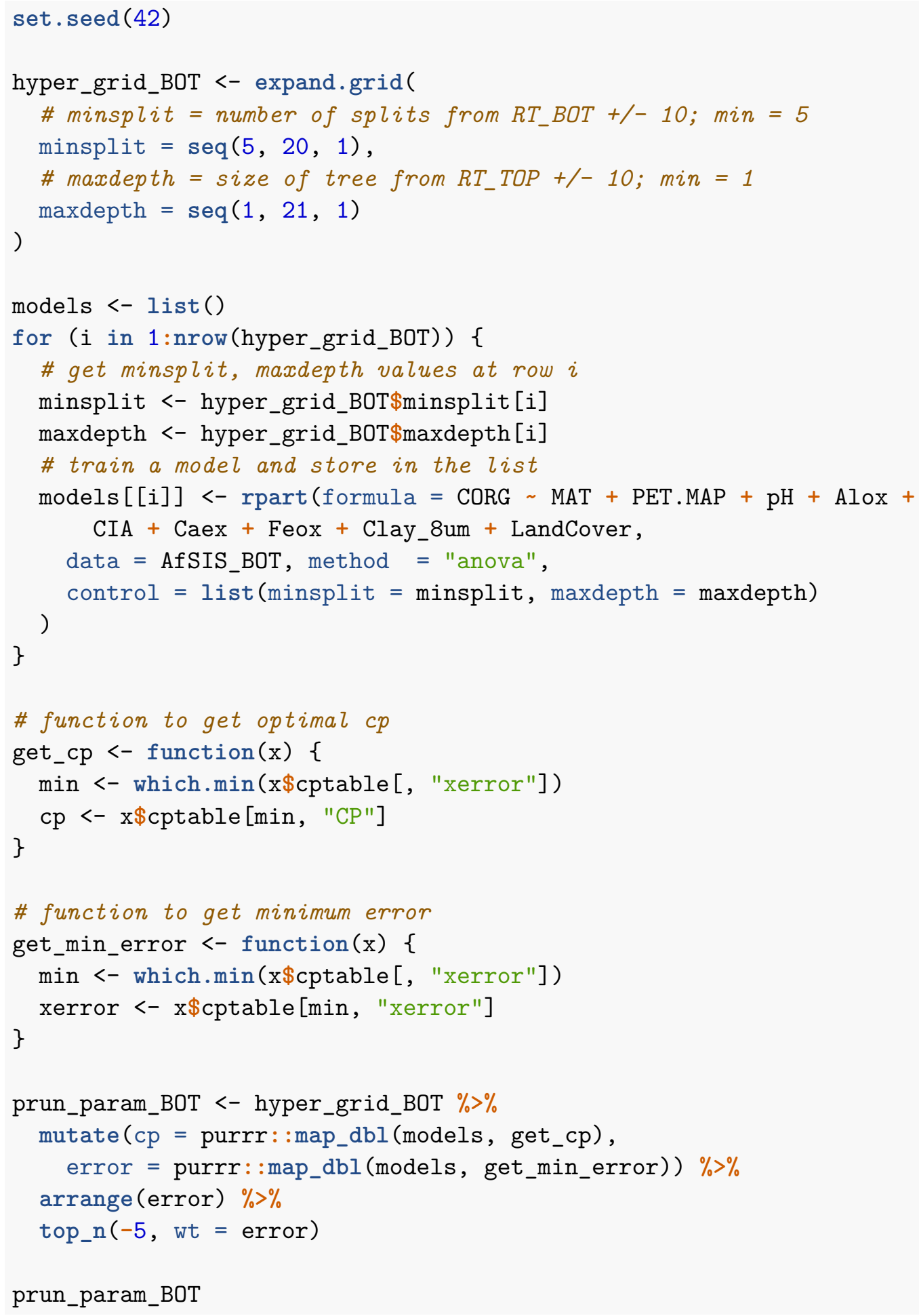




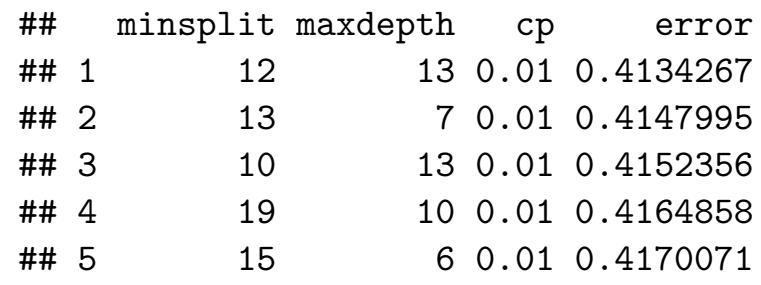

Pruned regression tree:

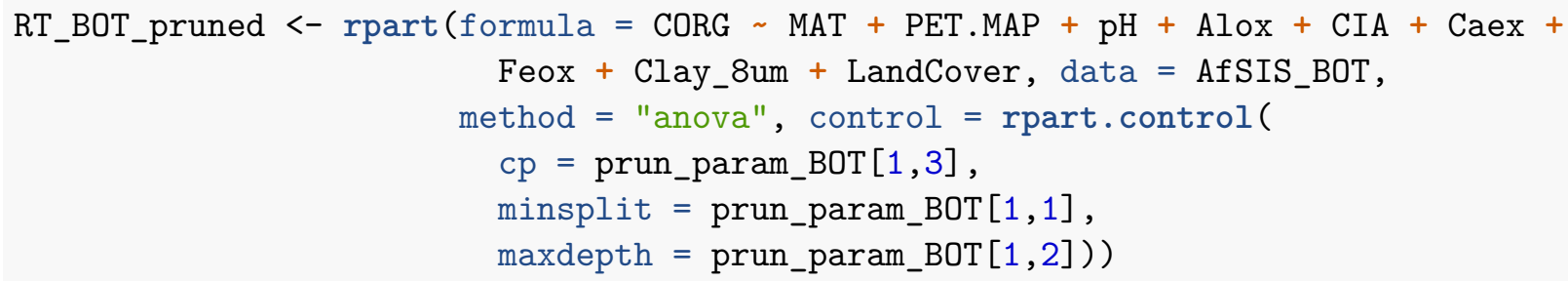

\section{Plotting:}

Figure $A 5$ in the supplement of the manuscript.

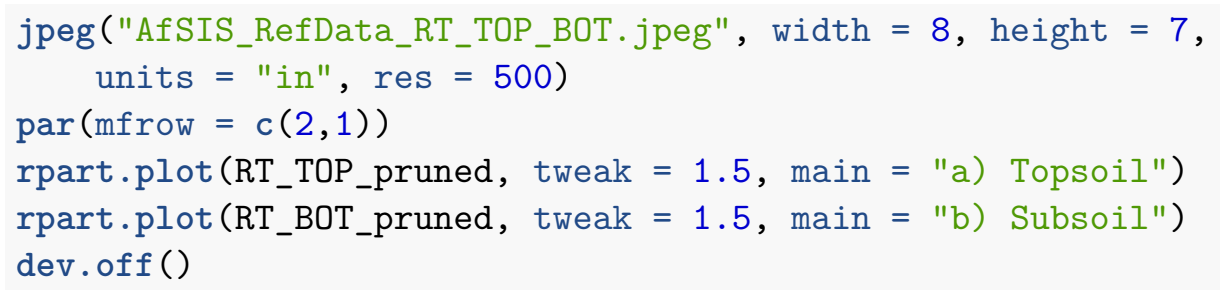

\subsection{Spatial cross-validation}

Code source: https://bookdown.org/robinlovelace/geocompr/spatial-cv.html Extract coordinates:

coords_TOP $<-$ AfSIS_TOP [,c("Longitude", "Latitude")]
coords_BOT $<-$ AfSIS_BOT[,c("Longitude", "Latitude")]

Select response and predictor variables:

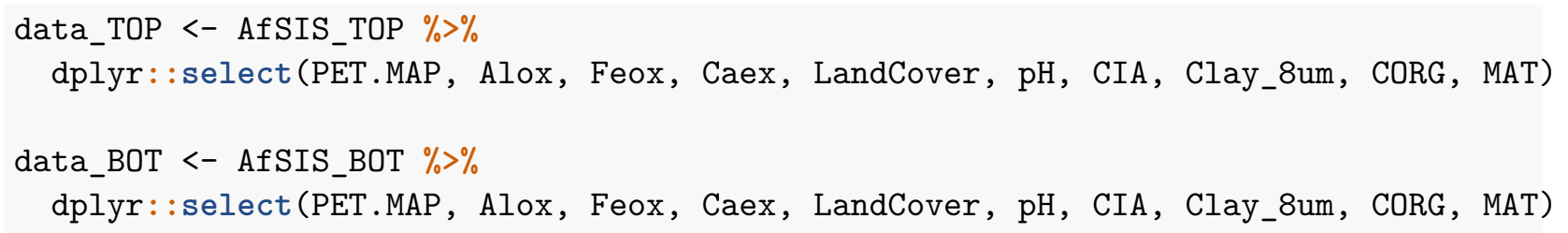

Create tasks: 
task_TOP_RT <- mlr: :makeRegrTask (data $=$ data_TOP, target $=$ "CORG", coordinates $=$ coords_TOP)

task_BOT_RT <- mlr: :makeRegrTask (data $=$ data_BOT, target $=$ "CORG", coordinates $=$ coords_BOT)

Create learners with tuning parameters from pruned regression tree:

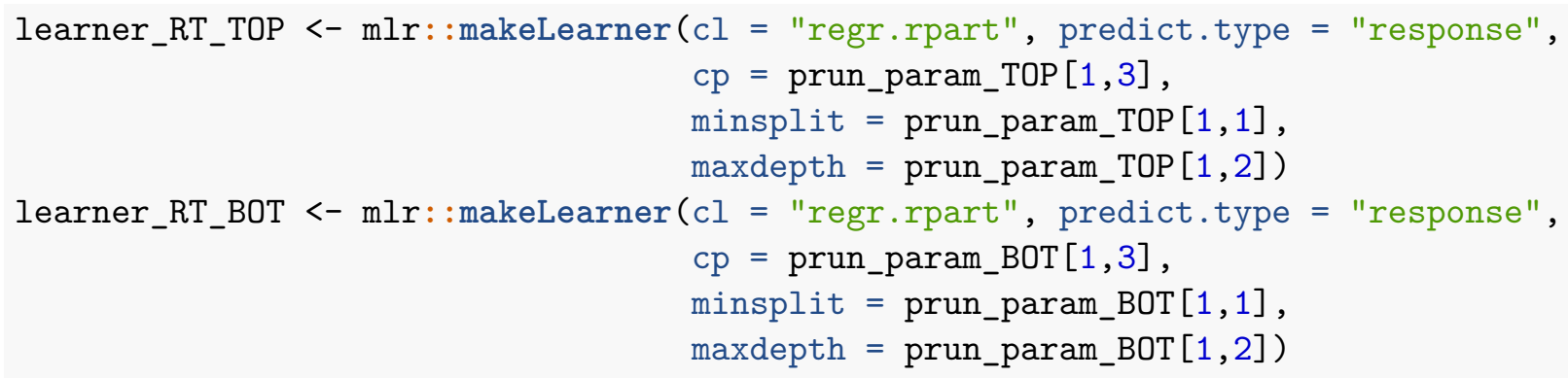

Define spatial partitioning:

rdesc <- mlr::makeResampleDesc(method = "SpRepCV", folds $=5$, reps $=100$ )

Resample: Topsoil

set.seed (42)

sp_cv_TOP_RT <- mlr: :resample(learner_RT_TOP, task_TOP_RT, resampling = rdesc, measures $=$ mlr::rmse, extract $=$ getLearnerModel, models $=$ TRUE)

\section{Summary RMSE and relative RMSE:}

summary (sp_cv_TOP_RT\$measures.test\$rmse)

\#\# Min. 1st Qu. Median Mean 3rd Qu. Max.

$\begin{array}{lllllll}\text { \#\# } & 0.7962 & 1.3713 & 1.4734 & 1.4897 & 1.7403 & 3.1142\end{array}$

\# relative RMSE

median (sp_cv_TOP_RT\$measures.test $\$$ rmse) $/$ mean (AfSIS_TOP\$CORG)

\#\# [1] 0.6482931

Resample: Subsoil

set.seed (42)

sp_cv_BOT_RT <- mlr: :resample(learner_RT_BOT, task_BOT_RT, resampling = rdesc, measures $=$ mlr::rmse, extract $=$ getLearnerModel, models $=$ TRUE $)$

Summary RMSE and relative RMSE: 
summary (sp_cv_BOT_RT\$measures.test\$rmse)

\#\# Min. 1st Qu. Median Mean 3rd Qu. Max.

$\begin{array}{lllllll}\# \# & 0.4398 & 0.5356 & 0.6715 & 0.9079 & 1.2313 & 2.2626\end{array}$

\# relative RMSE

median (sp_cv_BOT_RT\$measures.test\$rmse)/mean (AfSIS_BOT\$CORG)

\#\# [1] 0.4759412

\section{Plotting:}

Figure $A 3$ in the supplement of the manuscript.

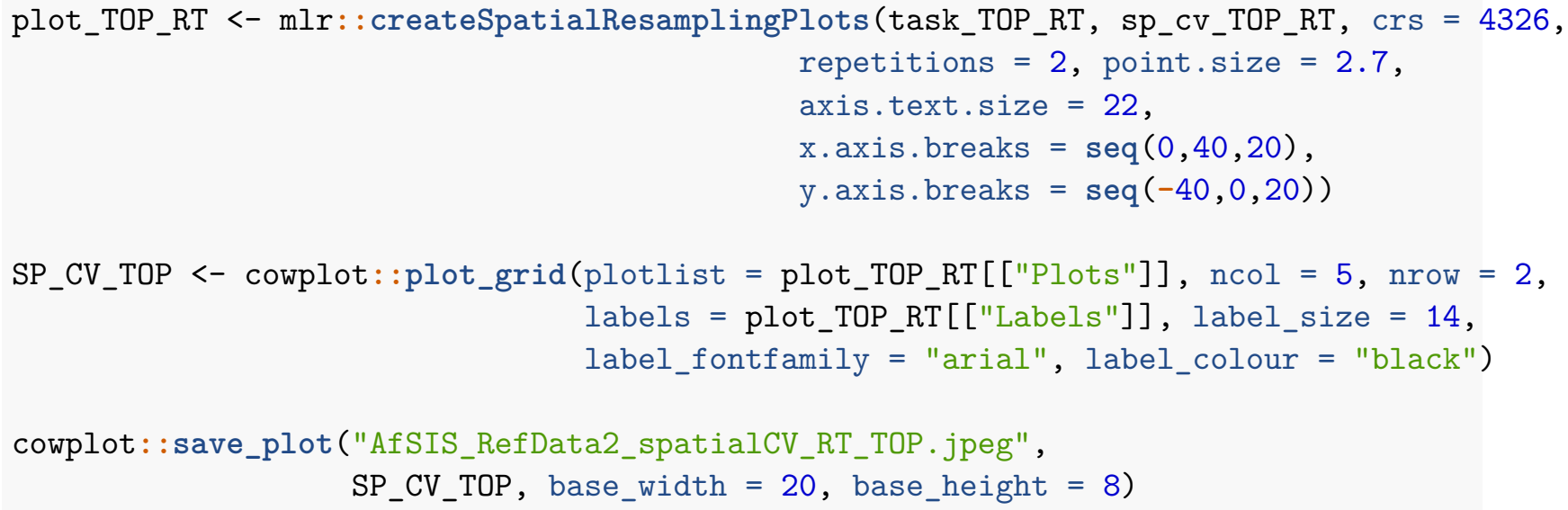

\section{Random forest}

This section contains all the code for the regression tree analyses and the partial dependence plots. Same as for the regression trees, the two depth layers were modelled sperately.

Code source: https://bookdown.org/robinlovelace/geocompr/eco.html

\subsection{Modelling}

\section{Topsoil:}

Filter dataset:

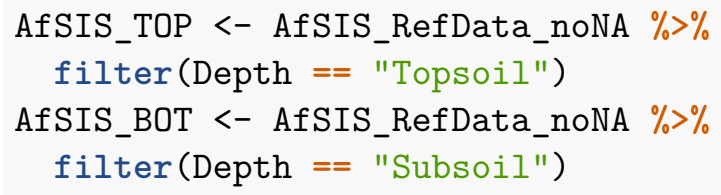




\section{Extract coordinates:}

coords_TOP <- AfSIS_TOP[,c("Longitude", "Latitude")]

coords_BOT <- AfSIS_BOT[,c("Longitude", "Latitude")]

Select response and predictor variables (also for the clay- and land cover-only models):

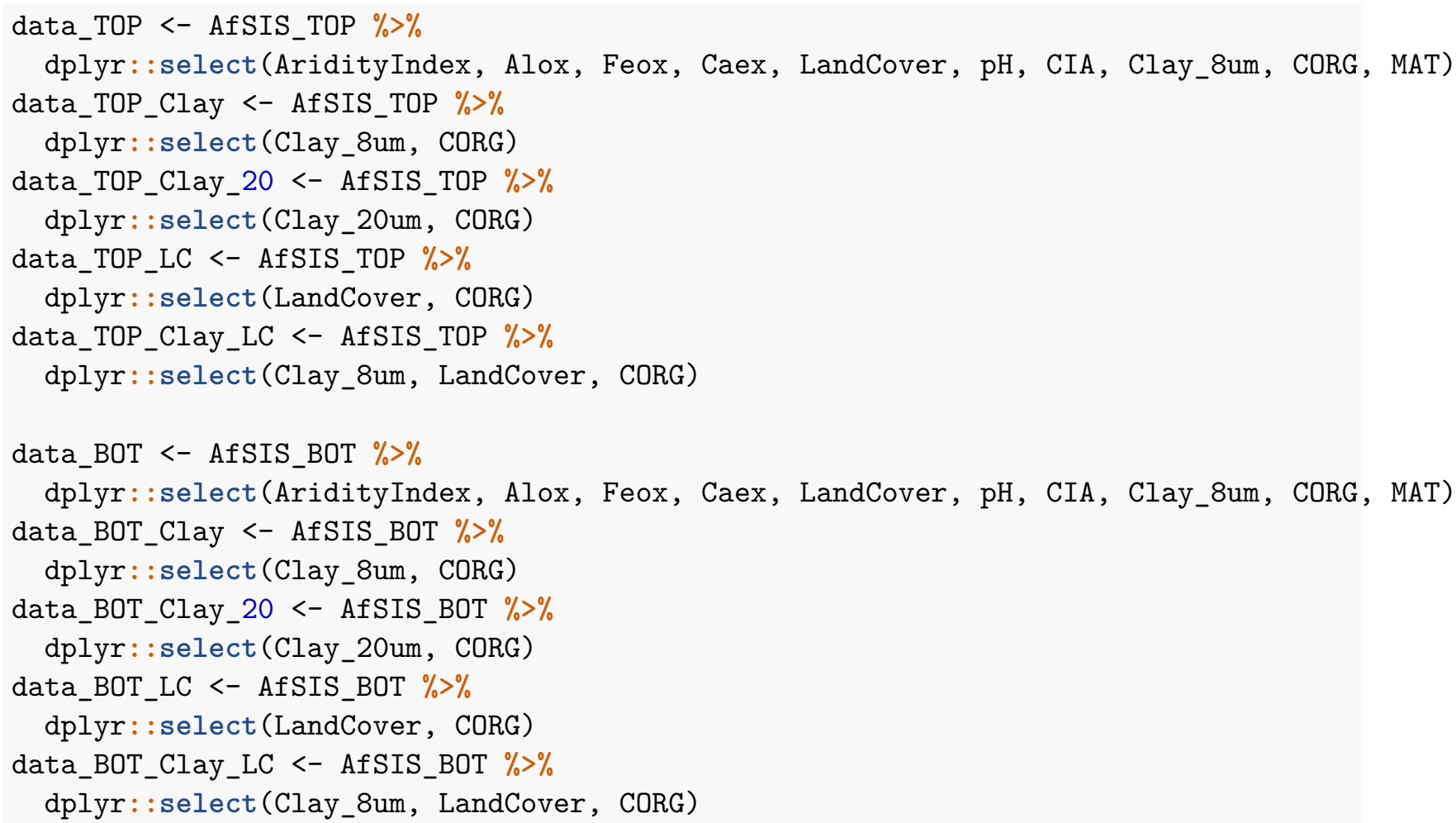

\section{Create tasks:}

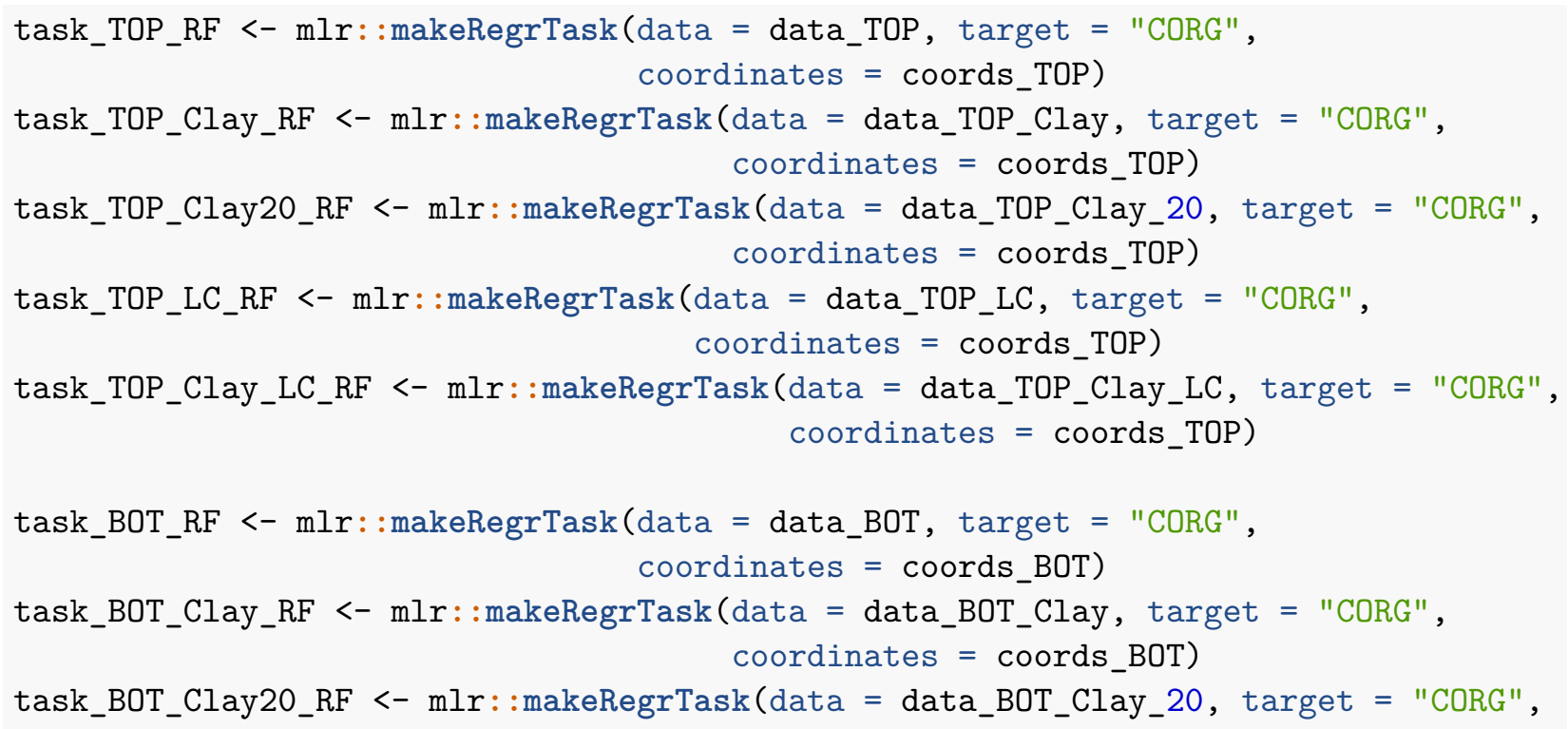




coordinates $=$ coords_BOT $)$
task_BOT_LC_RF <- mlr: :makeRegrTask $($ data $=$ data_BOT_LC, target $=$ "CORG",
coordinates $=$ coords_BOT)
task_BOT_Clay_LC_RF <- mlr: :makeRegrTask(data $=$ data_BOT_Clay_LC, target = "CORG",
coordinates $=$ coords_BOT)

Create learner (based on the ranger $\mathrm{R}$ package):

lrn_rf <- mlr::makeLearner (cl = "regr.ranger", predict.type = "response")

Specifiy resampling (5 spatially disjoint partitions):

tune_level = mlr::makeResampleDesc ("SpCV", iters $=5$ )

Specify number of random search:

ctrl <- mlr::makeTuneControlRandom(maxit $=50 \mathrm{~L})$

The search for the best hyperparameters will be done 50x in each of the spatial disjoint partitions. This allows to take the clustering of the samples into account.

Specify hyperparameter search:

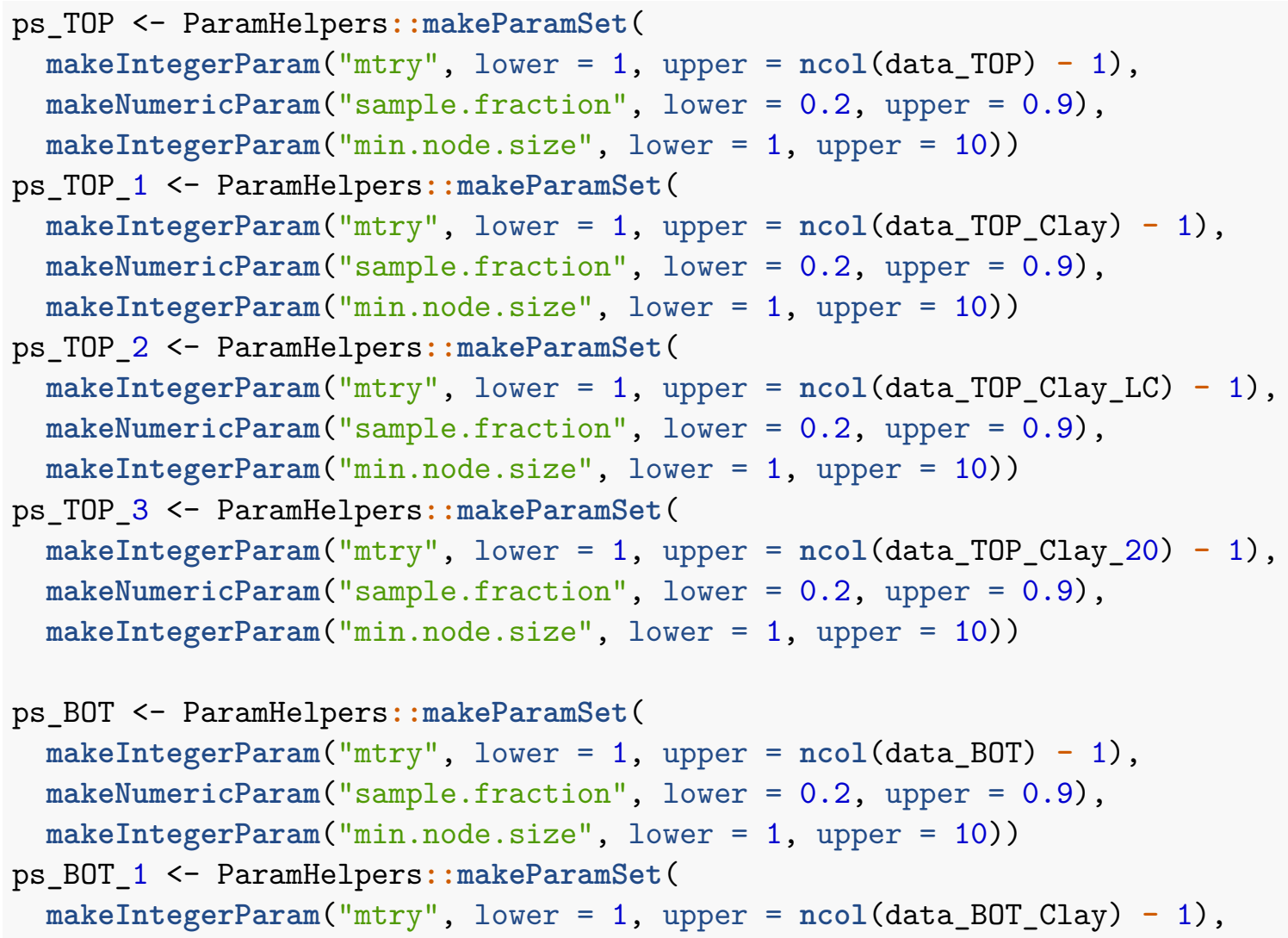




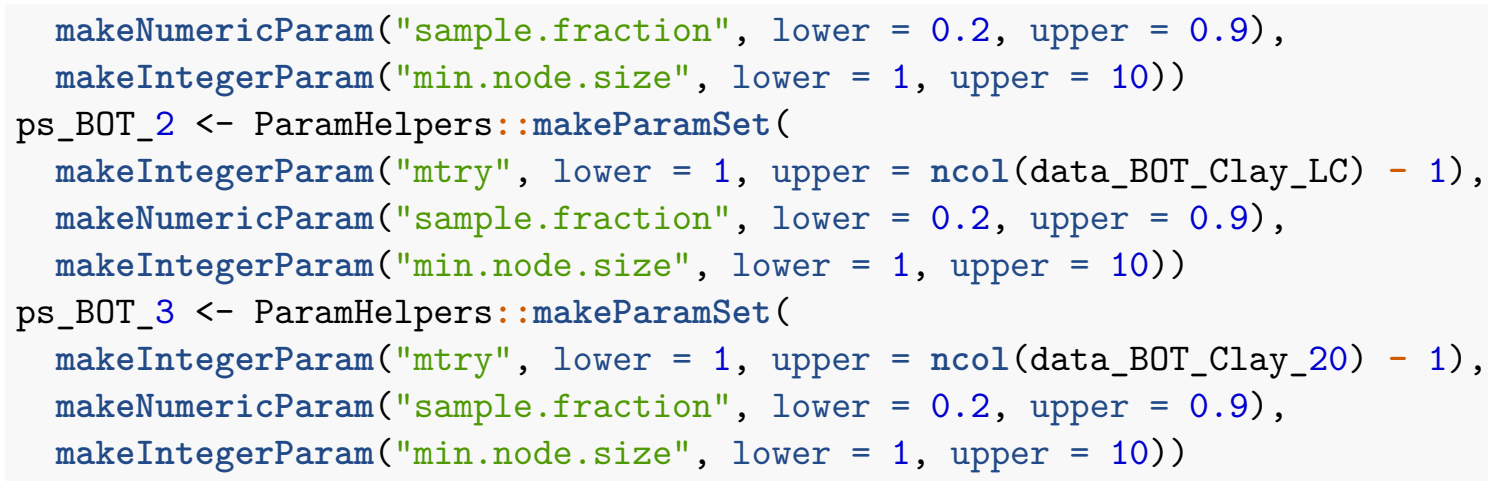

\section{Tuning: Topsoil}

Full model:

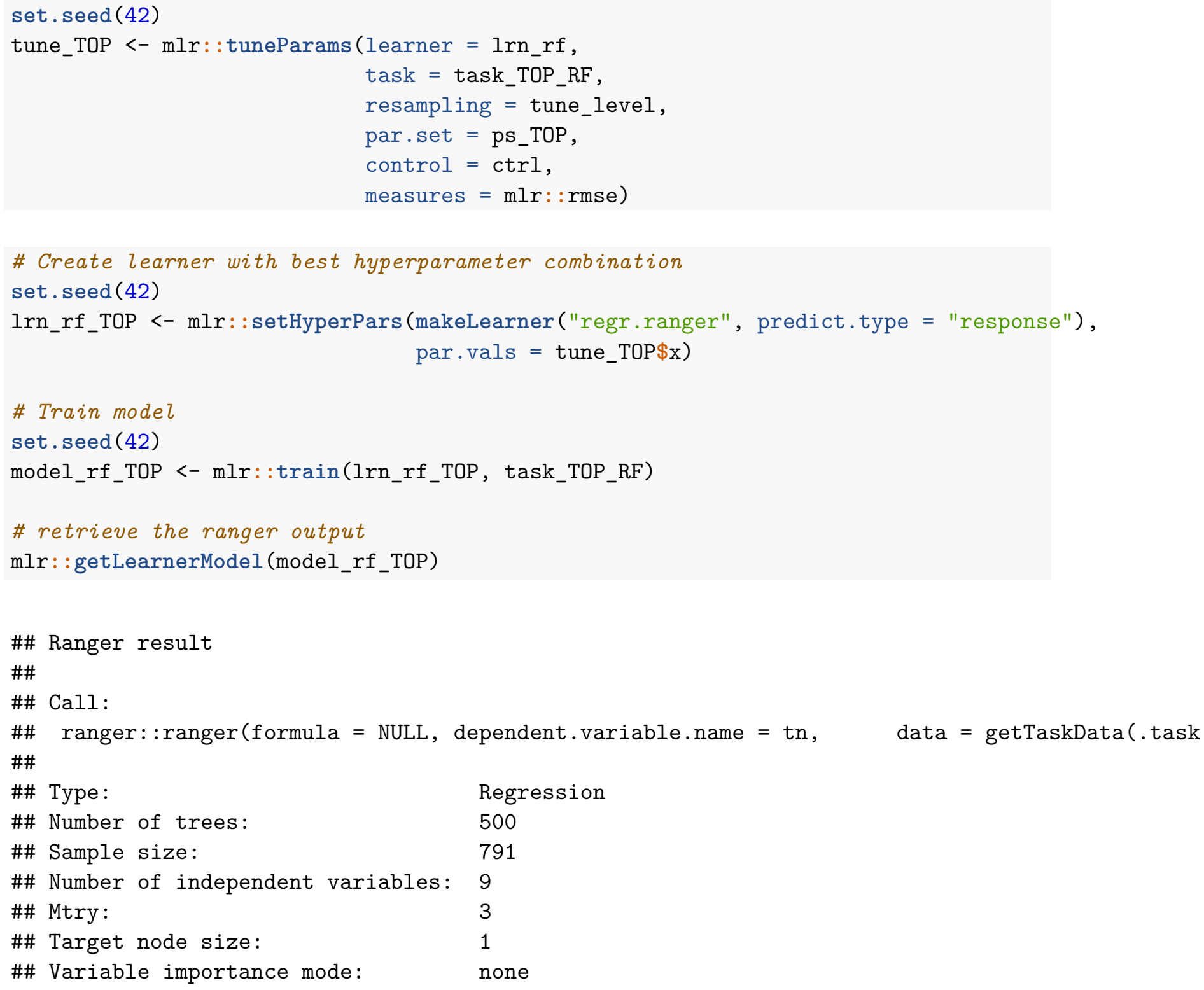


\#\# Splitrule:

\#\# OOB prediction error (MSE):

\#\# R squared (OOB): variance

0.8732137

0.7022975

Clay-only model (<8um):

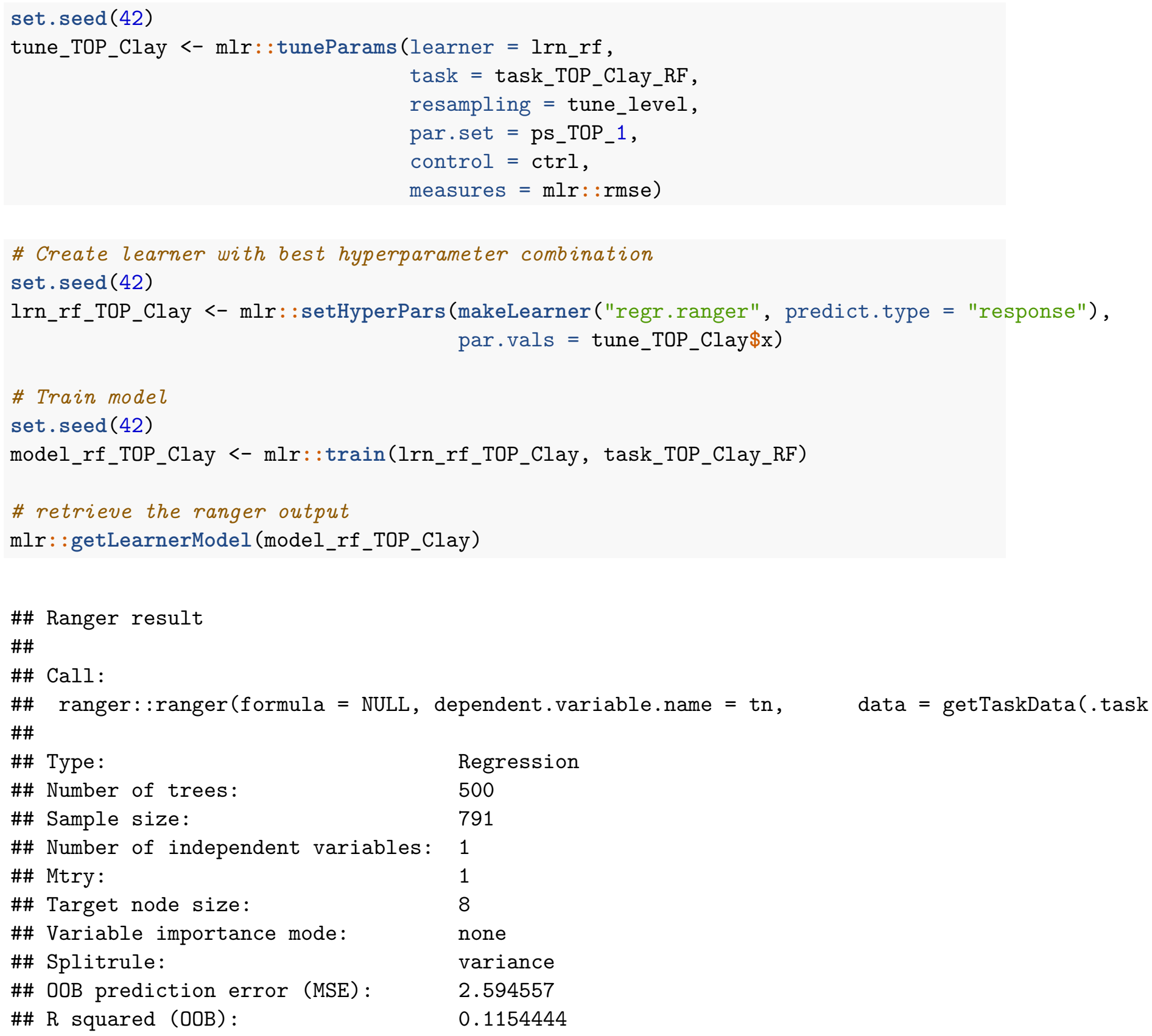

Clay-only model (<20um):

set. seed (42)

tune_TOP_Clay20<- mlr: :tuneParams (learner = lrn_rf, 


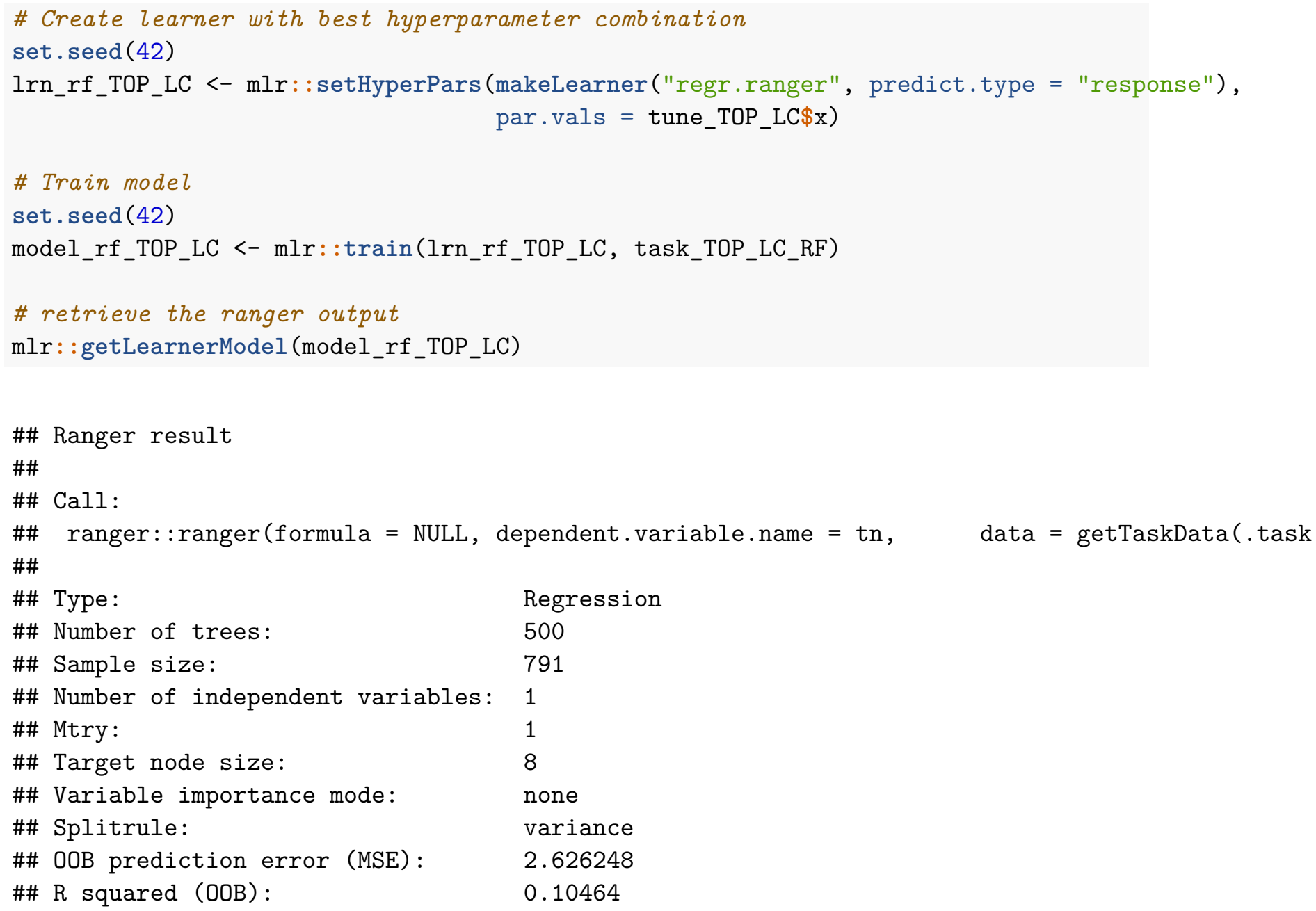

Clay + land cover model:

set. seed (42)

tune_TOP_Clay_LC <- mlr: :tuneParams (learner = lrn_rf, task = task_TOP_Clay_LC_RF, resampling = tune_level, 


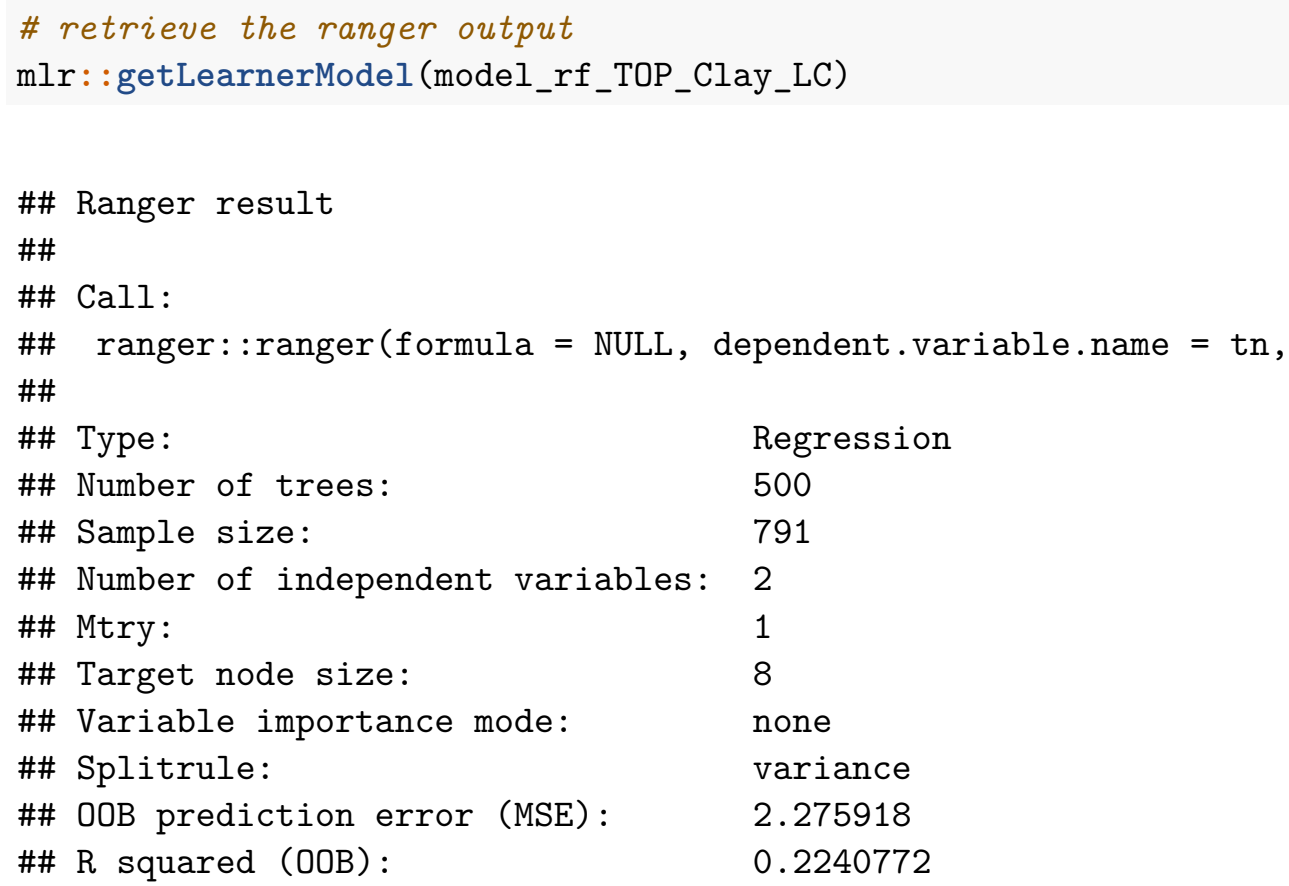

\section{Tuning: Subsoil}

Full model:

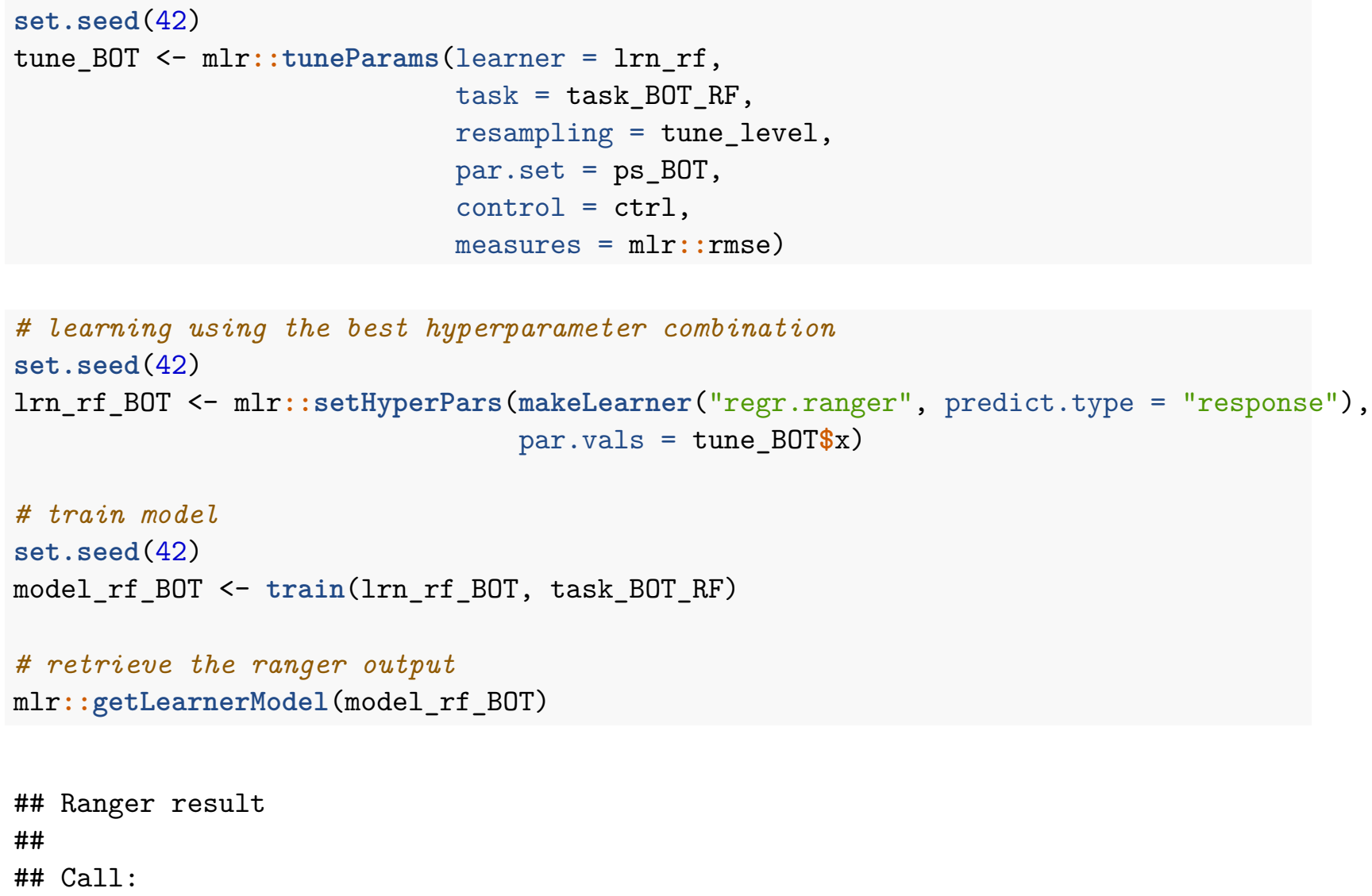




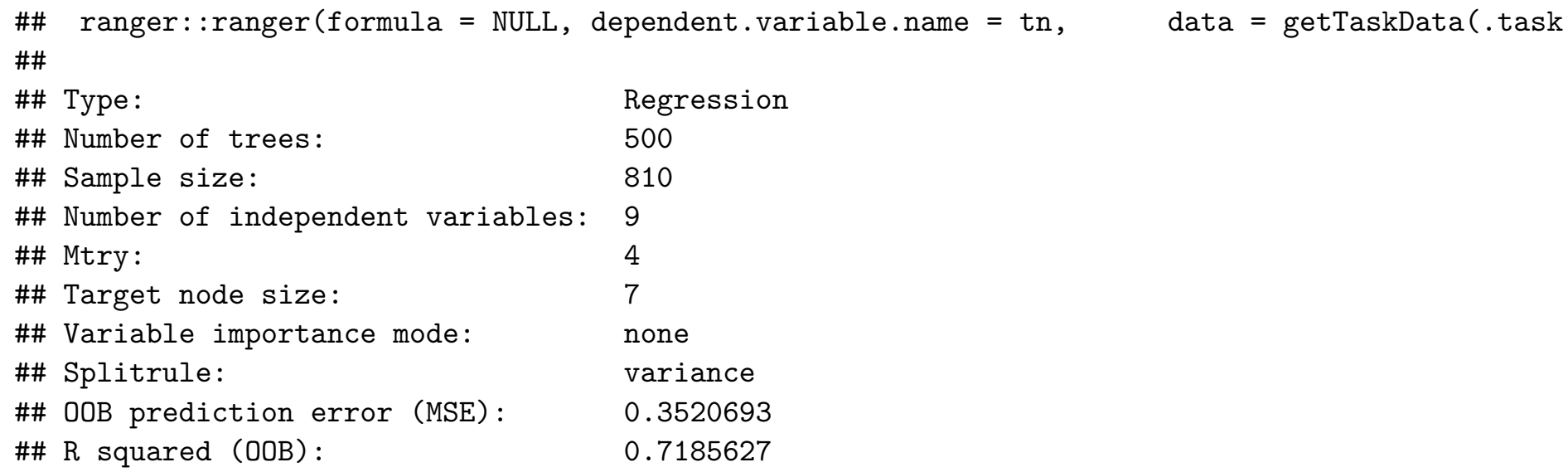

Clay-only model (< 8um):

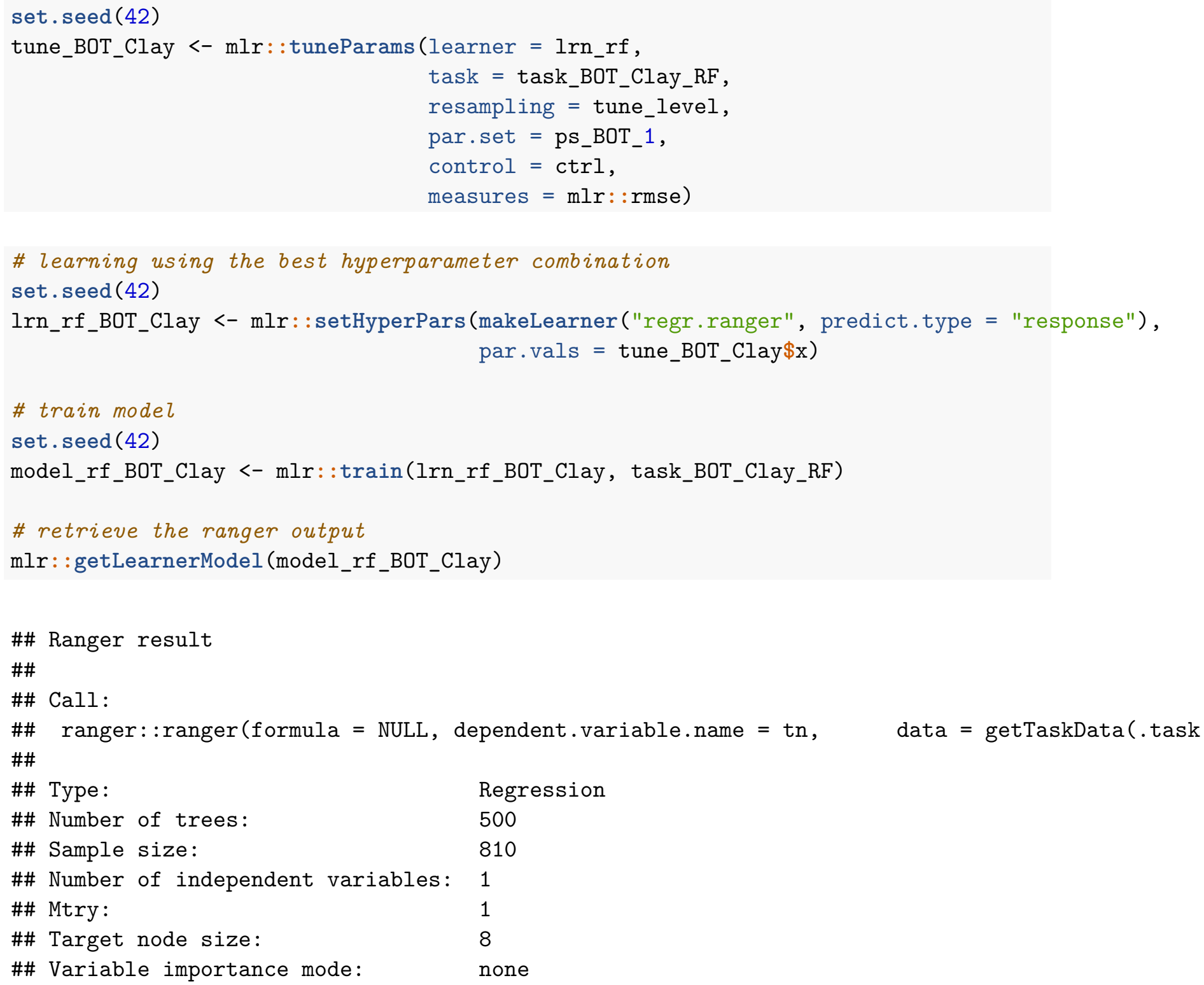




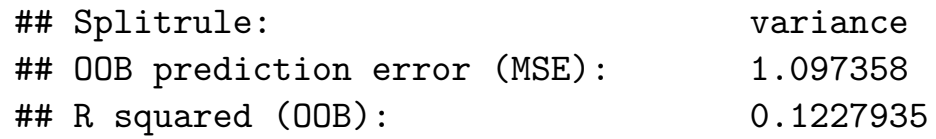

Clay-only model (<8um):

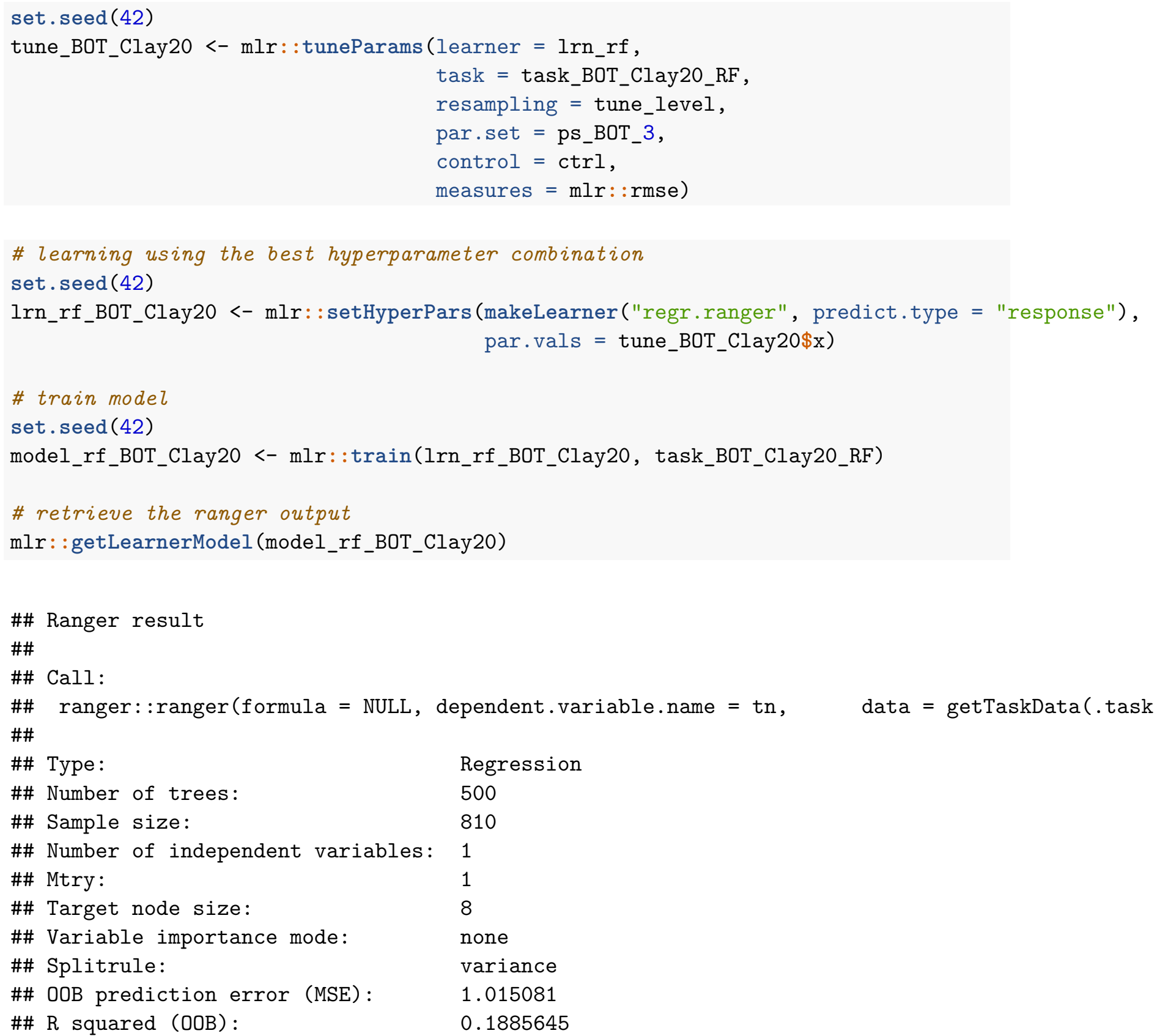

Land-cover-only model:

set. seed (42)

tune_BOT_LC <- mlr: :tuneParams (learner = lrn_rf, 


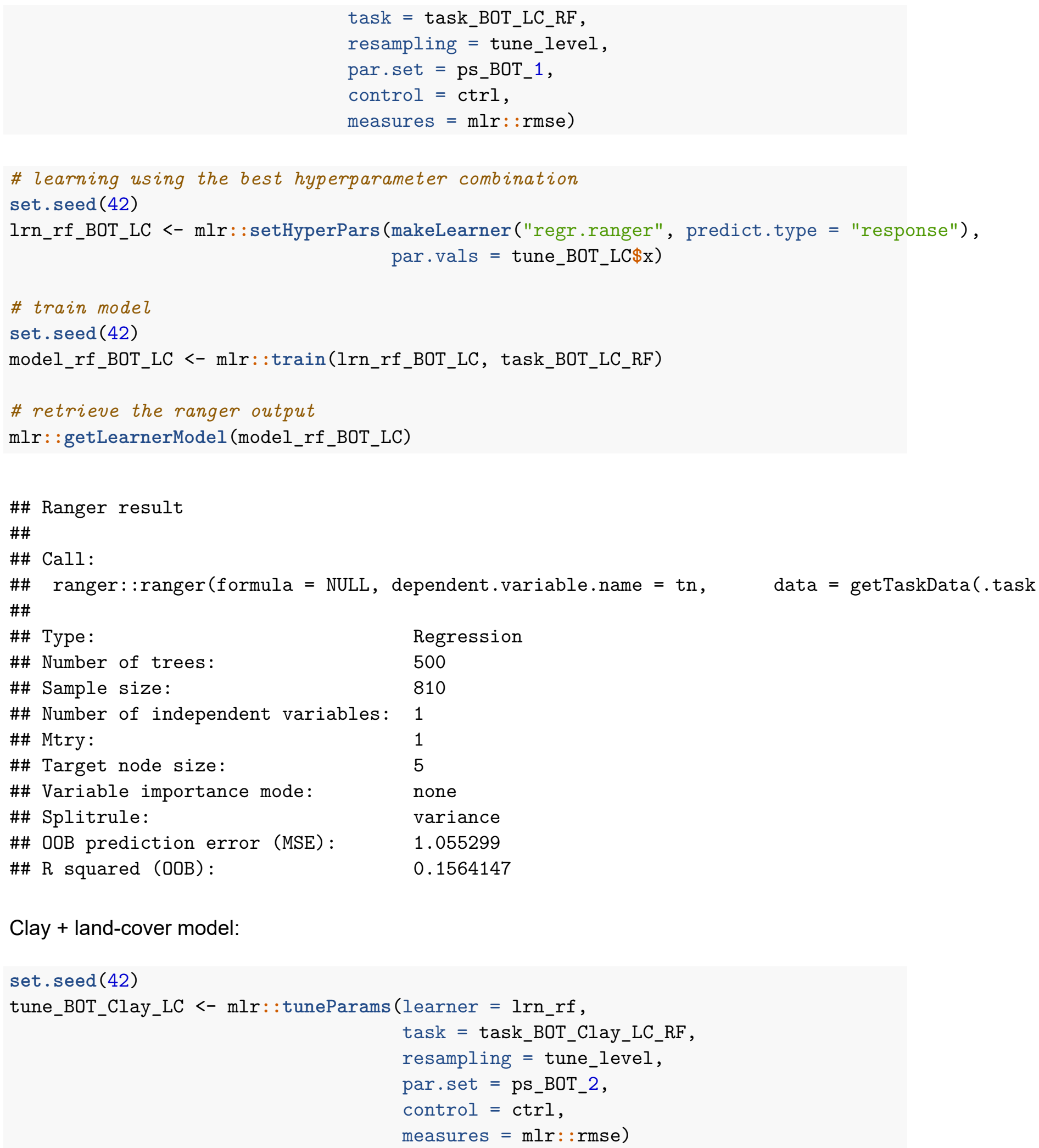

Clay + land-cover model:

set.seed (42)

tune_BOT_Clay_LC <- mlr: : tuneParams (learner = lrn_rf, task = task_BOT_Clay_LC_RF, resampling = tune_level, 


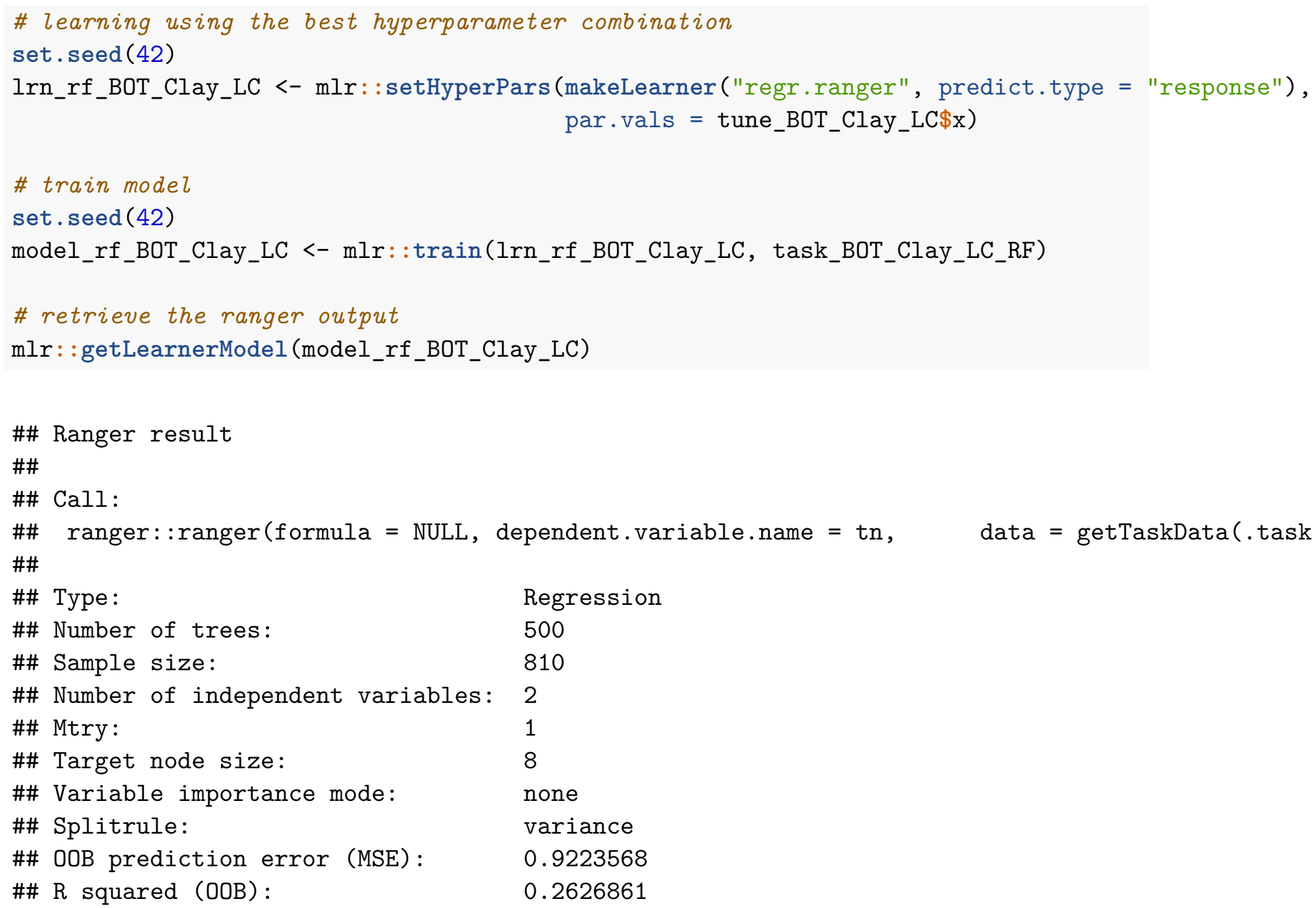

\subsection{Partial Dependence Plots}

Figure 6 in the manuscript.

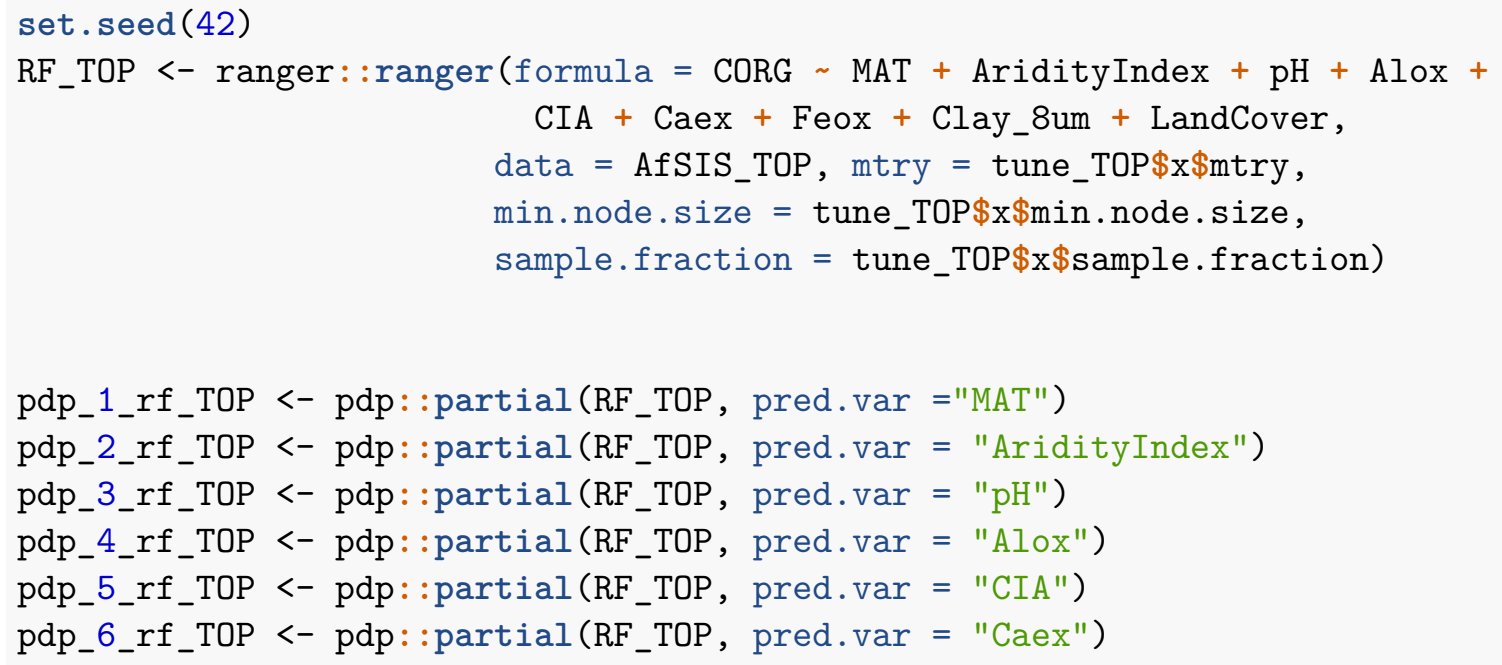




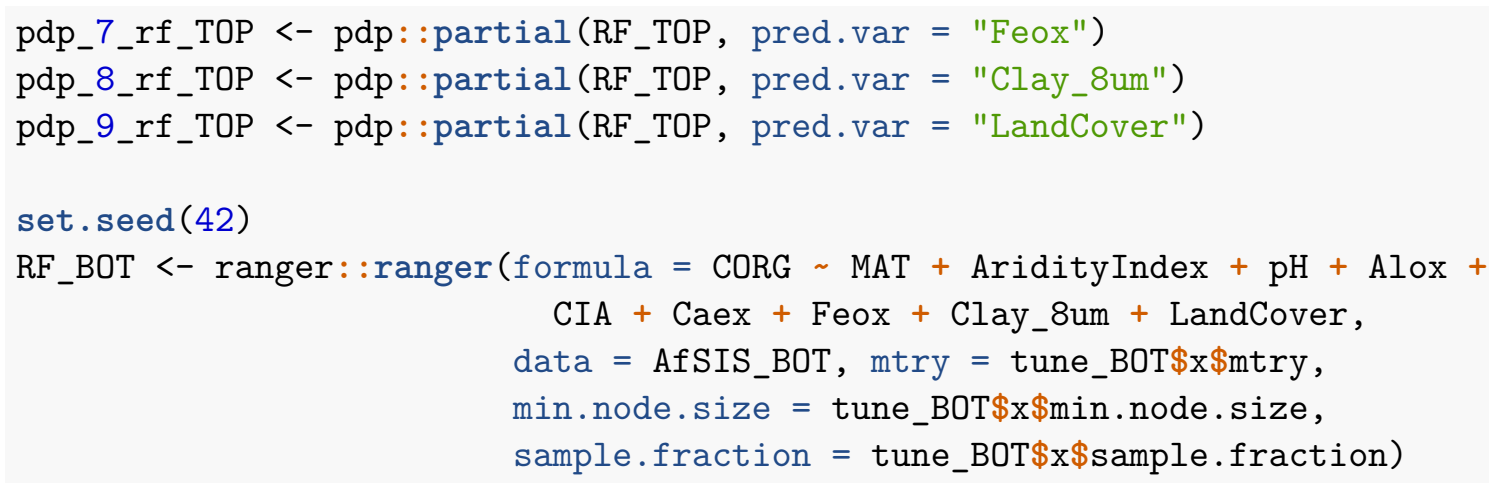

pdp_1_rf_BOT <- pdp: :partial $\left(R_{-}\right.$_BOT, pred.var = "MAT")

pdp_2_rf_BOT <- pdp: :partial (RF_BOT, pred.var = "AridityIndex")

pdp_3_rf_BOT <- pdp: :partial $\left(\mathrm{RF}_{-}\right.$BOT, pred.var $=$"pH")

pdp_4_rf_BOT <- pdp: partial $\left(\mathrm{RF} \_B O T\right.$, pred.var = "Alox")

pdp_5_rf_BOT <- pdp: :partial $($ RF_BOT, pred.var = "CIA")

pdp_6_rf_BOT <- pdp: :partial $\left(\mathrm{RF} \_B O T\right.$, pred.var = "Caex")

pdp_7_rf_BOT <- pdp: partial $\left(\mathrm{RF} \_B O T\right.$, pred.var = "Feox")

pdp_8_rf_BOT <- pdp: :partial (RF_BOT, pred.var = "Clay_8um")

pdp_9_rf_BOT <- pdp: partial $\left(\mathrm{RF}_{-}\right.$BOT, pred.var = "LandCover")

\section{Plotting:}

Partial dependence plot: Figure 5 in the manuscript.

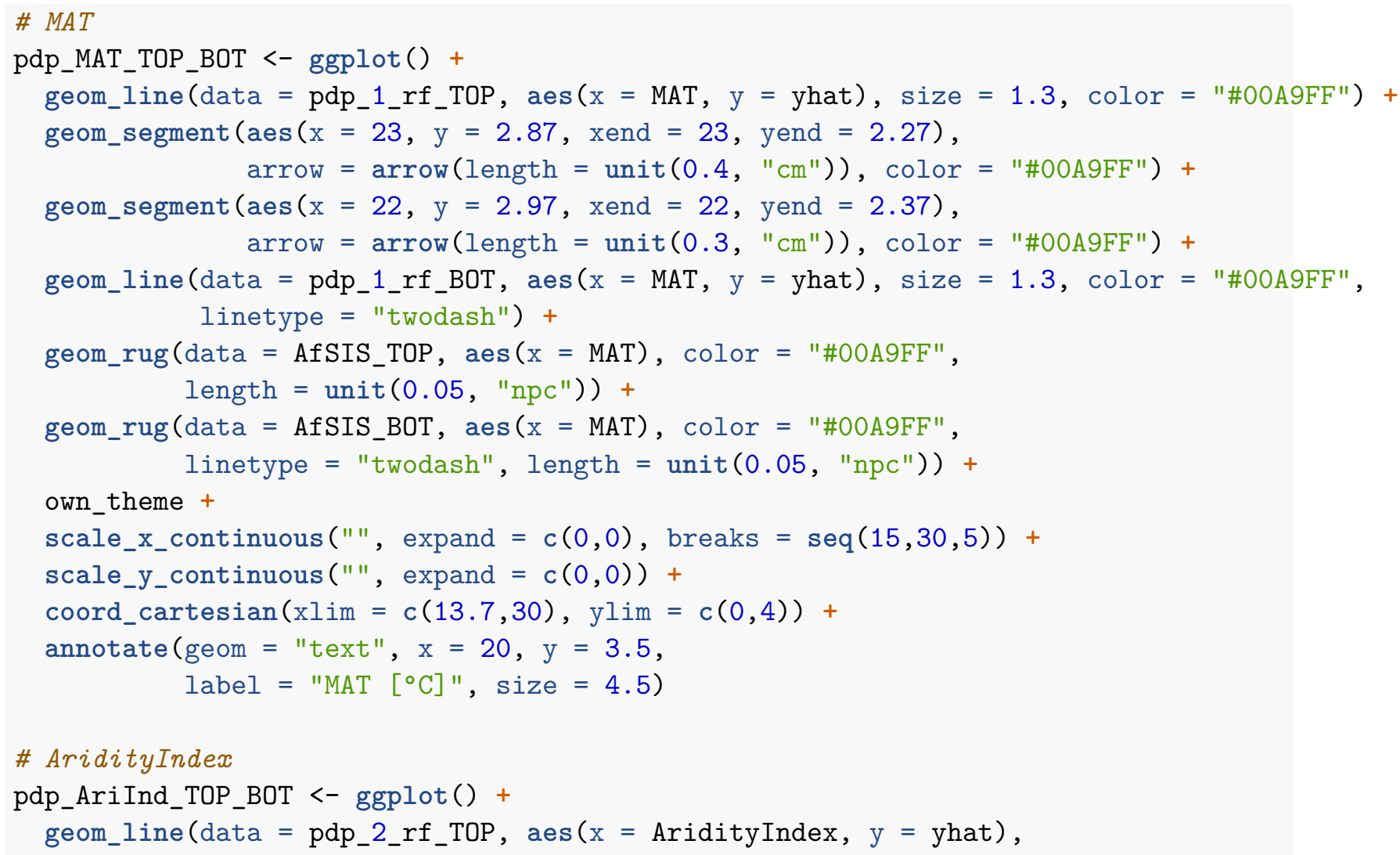




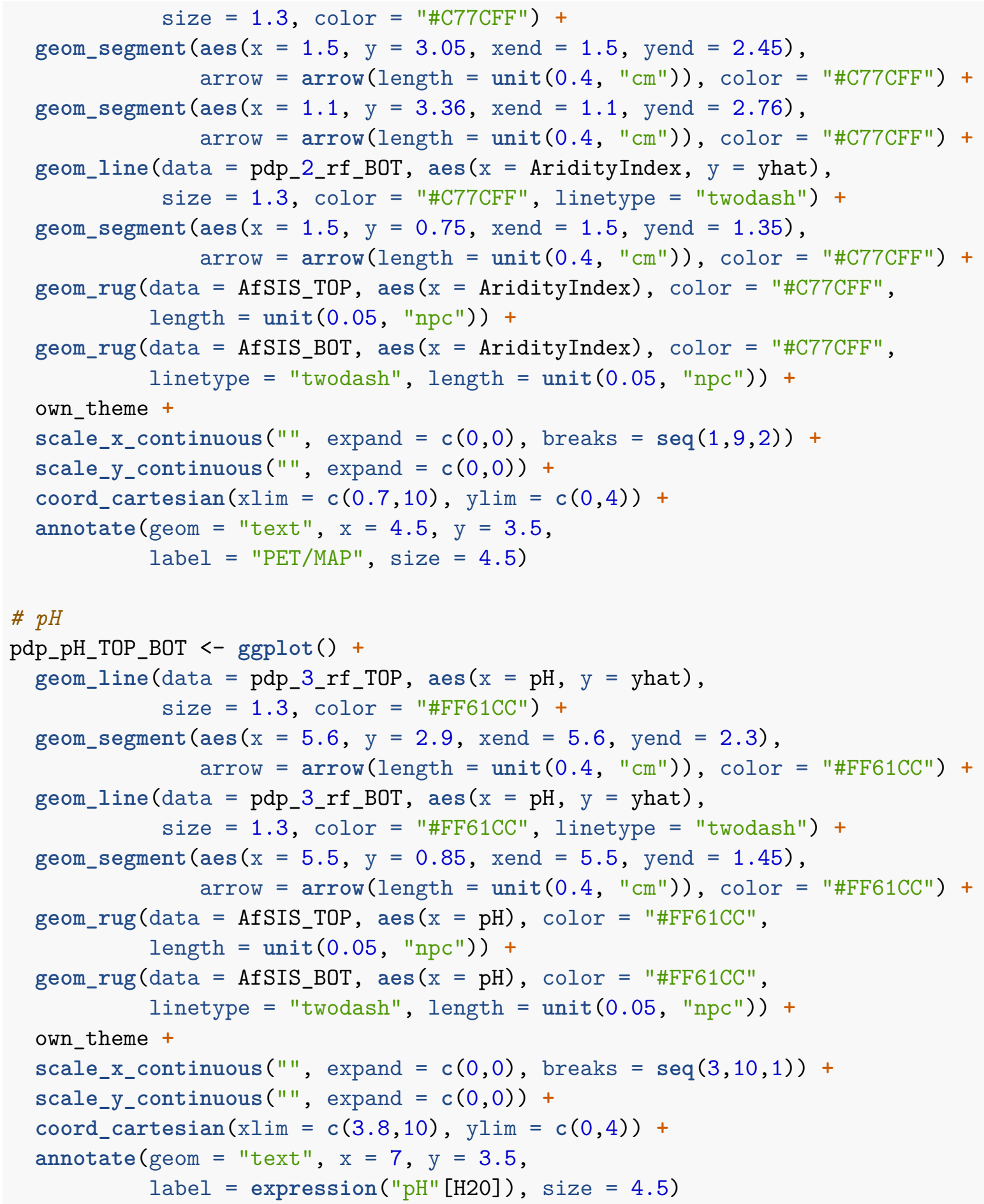




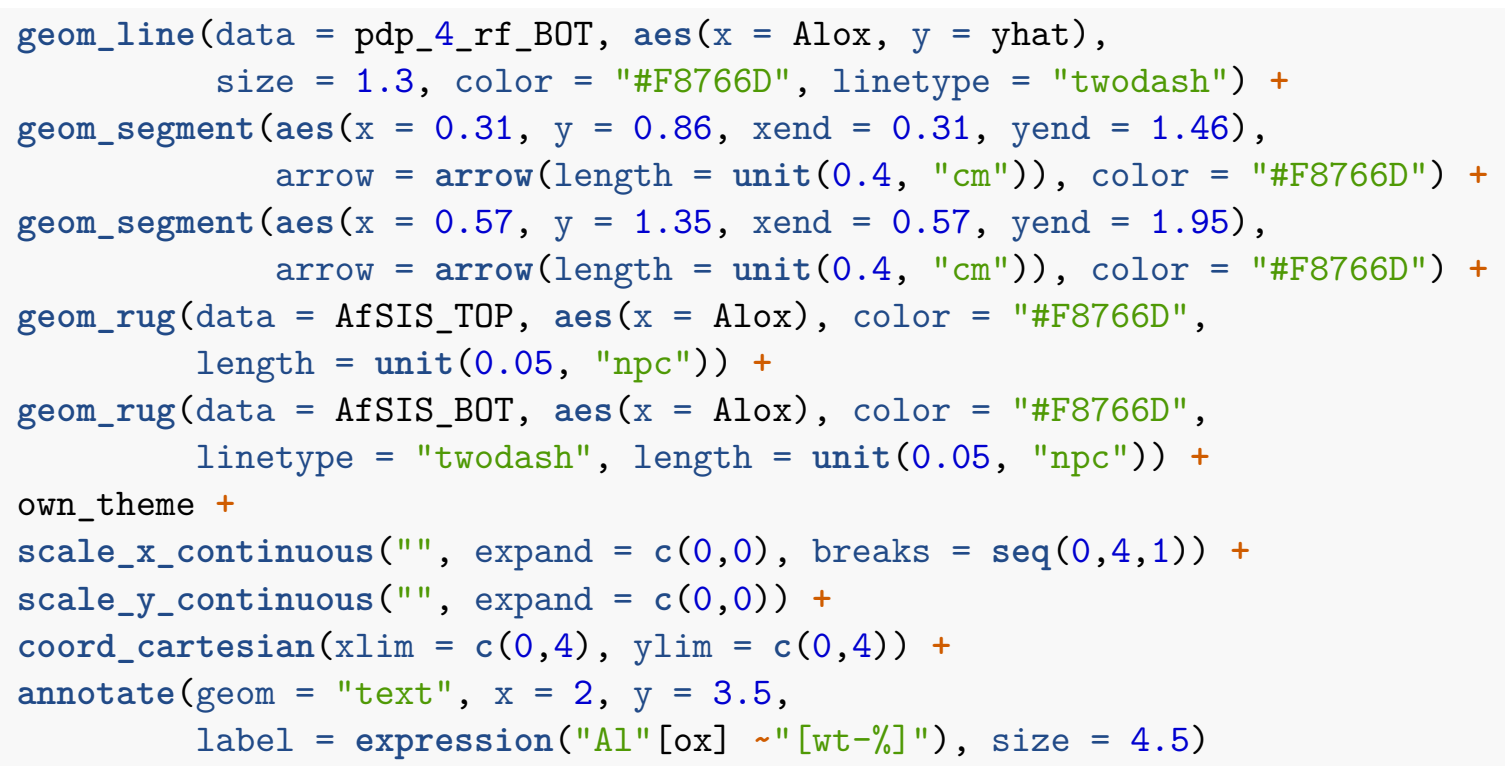




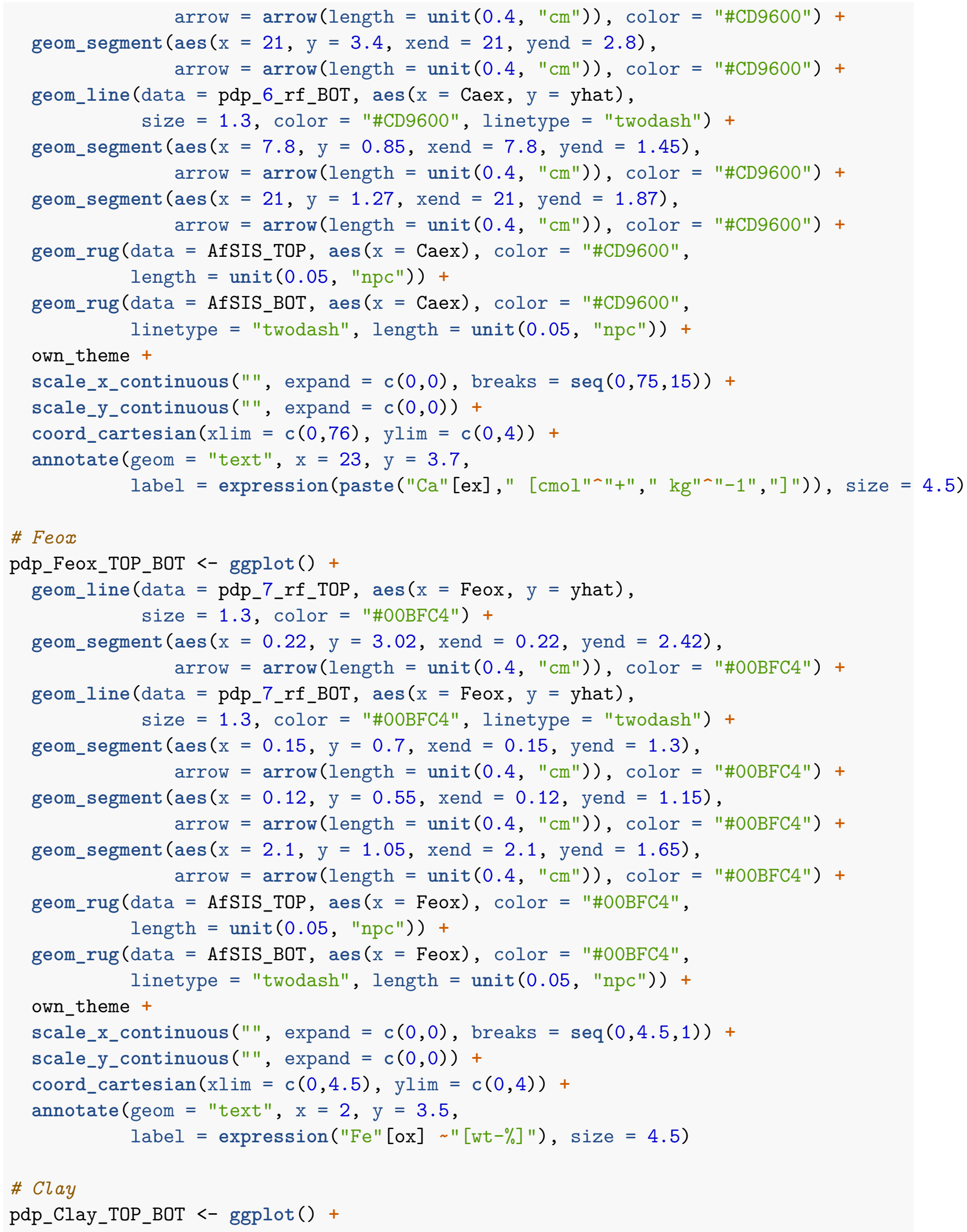


"e)", "f)", "g)", "h)",

"i)"))

annotate_figure(PDP_arranged,

left = text_grob("Predicted SOC content $[w t-\%] "$, color = "black", rot $=90$, size $=14$, vjust $=0.7)$ )

\#Save

ggsave("AfSIS_RefData_PDP_TOP_BOT_RF.jpeg", width = 9, height = 7, dpi = 450) 